\title{
Evaluating time-dependent changes in food seeking following periods of forced abstinence and associated changes in the nucleus accumbens
}

\author{
By \\ Erin Wendy Noye Tuplin
}

A thesis submitted to the Faculty of Graduate and Postdoctoral Affairs in partial fulfillment of the requirements for the degree of

Doctor of Philosophy

in

Neuroscience

\author{
Carleton University \\ Ottawa, Ontario
}

(C) 2018

Erin Wendy Noye Tuplin 


\begin{abstract}
Incubation of craving is a phenomenon whereby responding for cues associated with reward (food and drugs) increases over extended periods of abstinence. Evaluating the mechanisms of food craving has implications in understanding how consumption of foods high in certain macronutrients (fat, sugar) may contribute to continued cravings and subsequent weight gain. The primary goals of this thesis were to examine if forced abstinence from different food rewards resulted in a continued/or delayed craving response by utilizing different behavioral designs (contingent vs non-contingent) and examining associated changes in nucleus accumbens (NAc) immediate early genes, dendritic spine density, and AMPA receptor protein expression. Food self-administration (operant conditioning), classical conditioning, and conditioned place preference (CPP) were utilize for the investigation of incubation of food craving. Abstinence from selfadministration of purified food pellets was the only food group to display an incubation of craving response. Self-administration of chocolate flavored pellet, sucrose, or chocolate sucrose pellets did not result in an incubation response but did result in consistent responding at all abstinence periods which was associated with elevated dendritic spine density for chocolate flavored pellets. The type of behavioral design affected association FosB labelling as rats that underwent classical conditioning displayed significantly lower FosB in the NAc compared to operant trained rats and food restricted controls. CPP for chocolate flavored pellets or milk chocolate chips showed that the type of food reward can affect the possibility of a place preference response, milk chocolate did not produce CPP. The CPP model was unable to produce an incubation of craving response which may have resulted from the rewarding properties of the food choice and number of training sessions. Exposure to rewarding cues rapidly increased GluR1 expression, but this was not specifically associated with an incubation of craving response, thus the functional significance has yet to be elucidated. The present thesis has highlighted that foods of different macronutrient composition and/or flavors and the use of different behavioral designs can affect the potential for an incubation of craving response and associated NAc changes.
\end{abstract}




\title{
Co-Authors
}

\author{
Matthew R. Holahan
}

Savannah H. M. Lightfoot

Madi T. Wright 


\section{Acknowledgements}

I would like to sincerely thank my supervisor, Dr. Matthew Holahan, for his direction and advice throughout my graduate school career. I would also like to thank my committee, Dr. Alfonzo Abizaid and Dr. Melissa Chee for their guidance. To Savannah Lightfoot, thank-you for your support with each of the experiments in this thesis. To my lab mates, thank you for being there for encouragement and support whenever it was needed. Special thanks my friends and family for their support and encouragement throughout this journey. 


\section{Dedication}

I would like to dedicate this thesis to my parents, Kimberly and Kenneth, who have only encouraged and supported me. Without their unending support this journey would not have been possible. 


\section{Abbreviations}

\begin{tabular}{|l|l|}
\hline AC & Adenylate cyclase \\
\hline AMPA & Alpha-amino-hydroxy-S-methyl-4-isoxazolepropionic \\
\hline AP-1 & Activator protein 1 \\
\hline BDNF & Brain-derived neurotrophic factor \\
\hline CA2+ & Calcium ion \\
\hline CAMKII & Calcium/calmodulin-dependent kinase II \\
\hline cAMP & Cyclic adenosine monophosphate \\
\hline CDK5 & Cyclin-dependent kinase 5 \\
\hline CI-AMPA & Calcium impermeable AMPA receptors \\
\hline CP-AMPA & Calcium permeable AMPA receptors \\
\hline CS & Conditioned Stimulus \\
\hline DA & Dopamine \\
\hline DSM-V & $\begin{array}{l}\text { Diagnostic and Statistical Manual of Psychiatric Disorders Edition } \\
5\end{array}$ \\
\hline EE & Environmental enrichment \\
\hline EMG & Electromyography \\
\hline fMRI & Functional magnetic resonance imaging \\
\hline GABA & Gamma-aminobutyric acid \\
\hline GDP & Guanosine diphosphate \\
\hline GRIK & G-protein regulated inwardly rectifying potassium channels \\
\hline GTP & Guanosine Triphosphate \\
\hline LTD & Long-term depression \\
\hline LTP & Long-term potentiation \\
\hline MG2+ & Magnesium ion \\
\hline MSN & Medium spiny neuron \\
\hline NA+ & Sodium ion \\
\hline NAc & Nucleus accumbens \\
\hline NAcC & Nucleus accumbens core \\
\hline NAcSh & Nucleus accumbens shell \\
\hline NMDA & N-methyl-D-aspartate \\
\hline PET & Positron emission tomography \\
\hline PFC & Prefrontal cortex \\
\hline PKA & Protein kinase A \\
\hline SE & Standard environment \\
\hline US & Unconditioned stimulus \\
\hline VTA & Ventral tegmental area \\
\hline YFAS & Yale Food Addiction Scale \\
\hline & \\
\hline
\end{tabular}




\section{Contents}

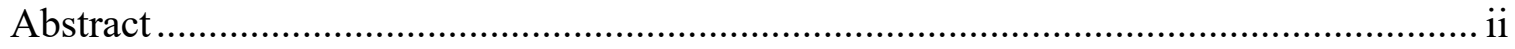

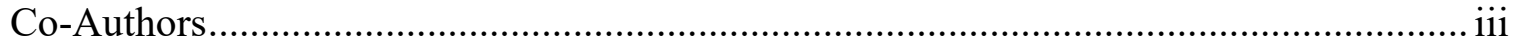

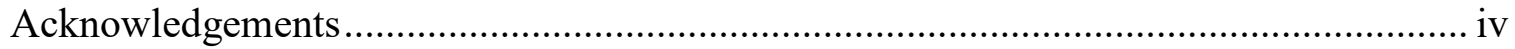

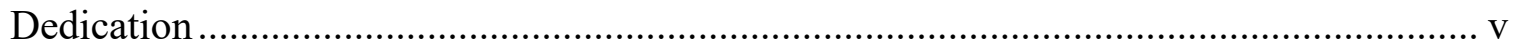

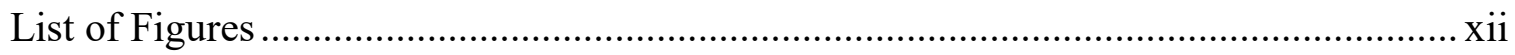

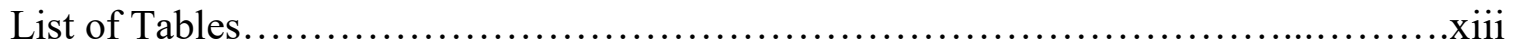

Preface

\section{Chapter 1: Introduction}

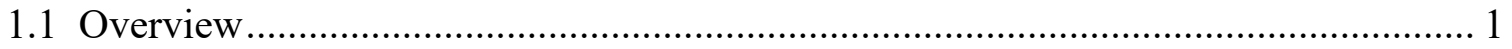

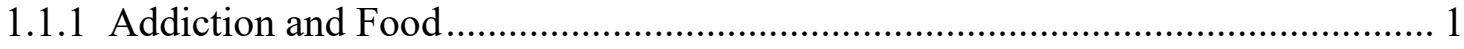

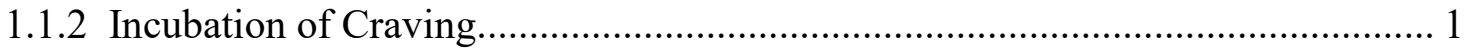

1.1.3 Food Craving ..................................................................................... 2

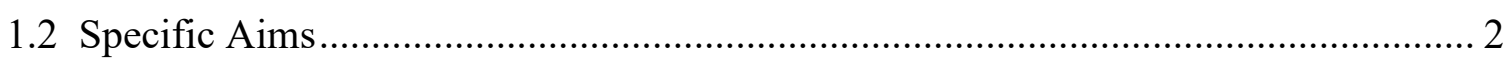

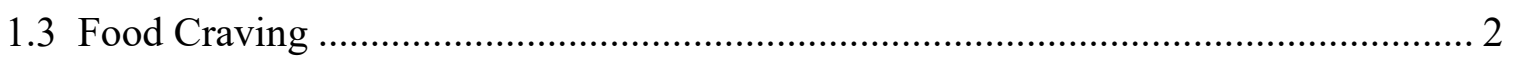

1.3.1 Importance of Studying Food Craving ......................................................... 3

1.3.2 Similarities Between Drug and Food Craving ................................................... 3

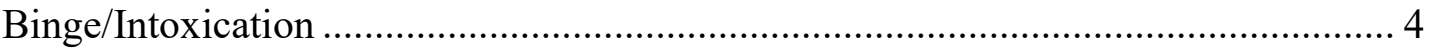

Withdrawal/Negative Affect................................................................................. 5

Preoccupation/Craving ........................................................................................ 6

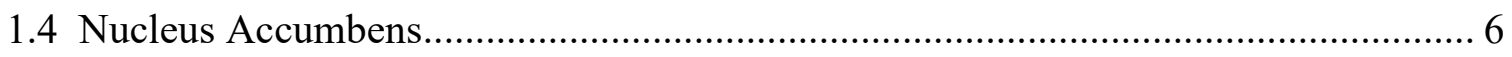

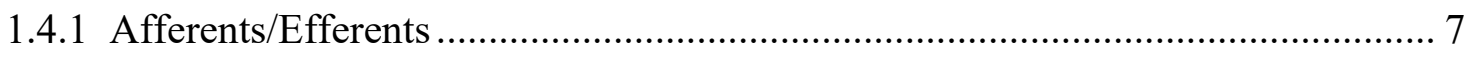

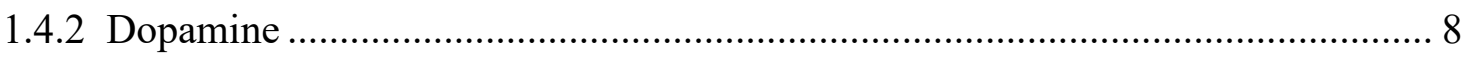

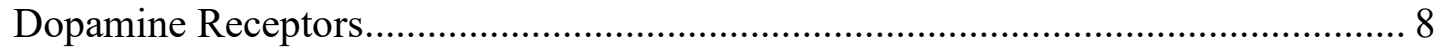

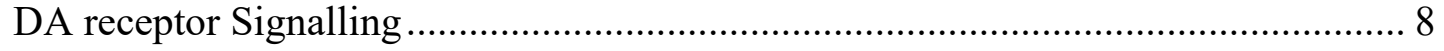

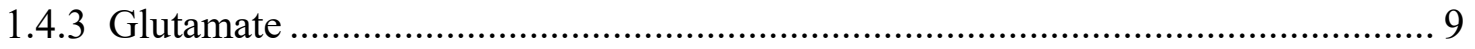

Ionotropic Glutamate Receptors ........................................................................ 9

AMPA receptor signalling ............................................................................ 10

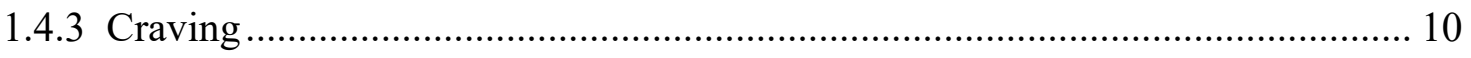

1.4.4 Immediate early genes.......................................................................... 12

Cfos 


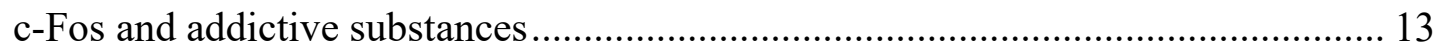

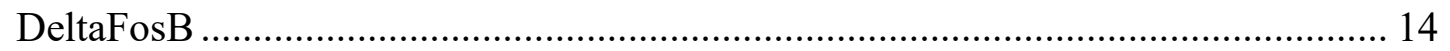

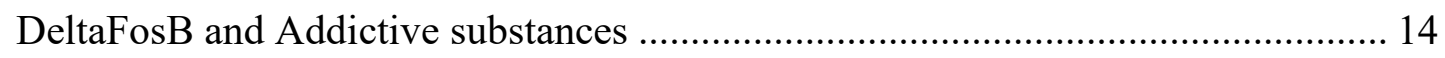

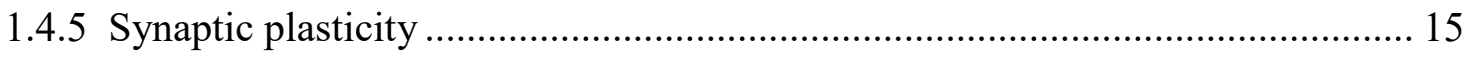

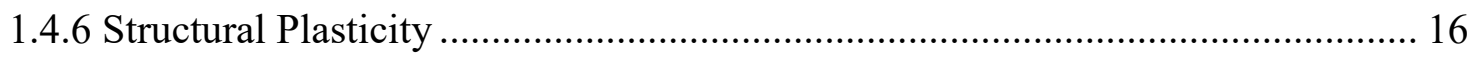

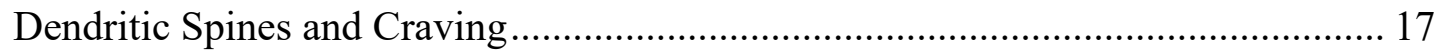

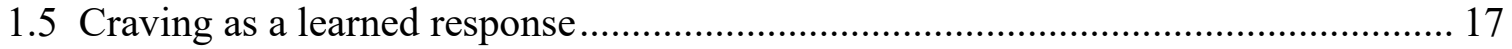

1.5.1 Classical Conditioning Principles ................................................................... 17

Classical Conditioning and Craving …........................................................... 18

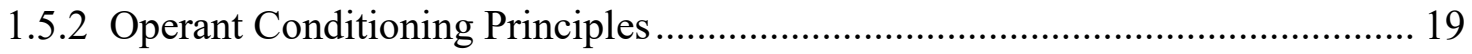

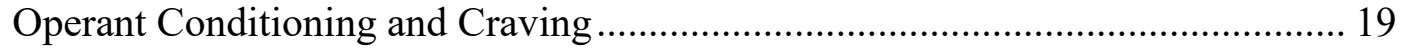

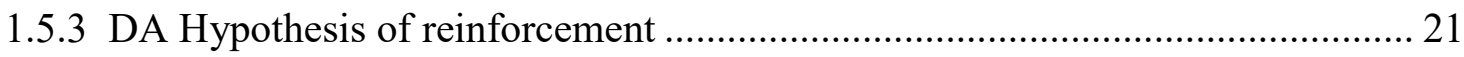

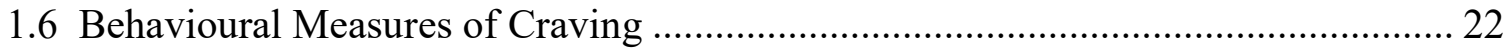

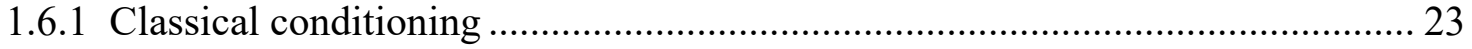

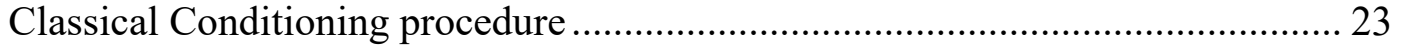

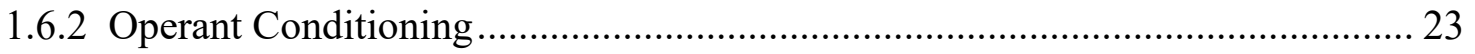

Operant Conditioning Procedure ................................................................... 23

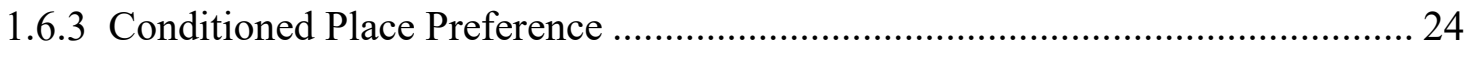

Conditioned Place Preference Procedure ........................................................... 24

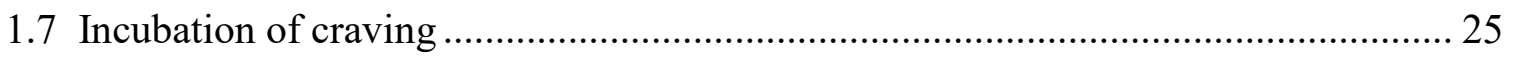

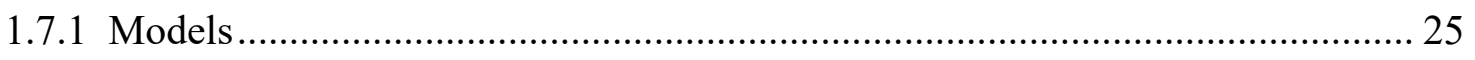

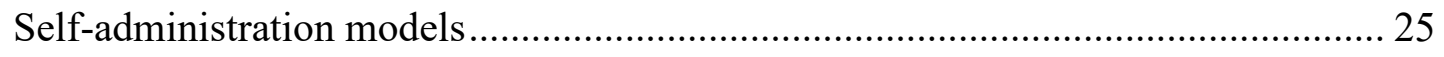

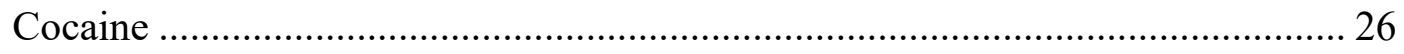

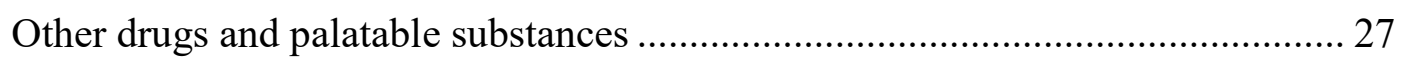

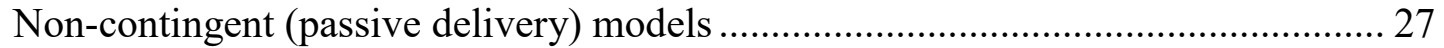

1.7.2 Experimental Manipulations (environmental enrichment) ............................. 28

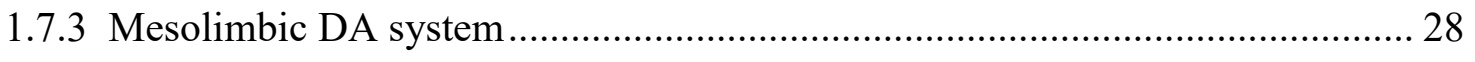

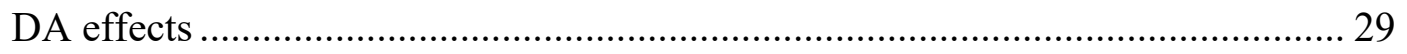

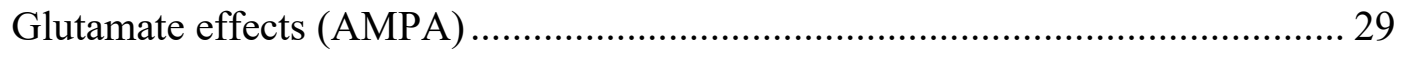

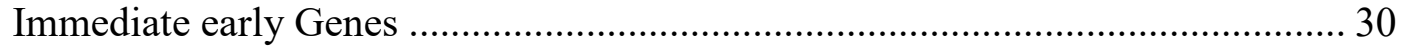

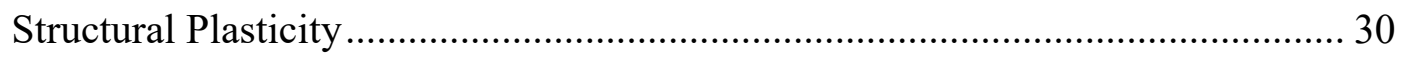


1.8 Current Thesis .

Chapter 2: Comparison of the Time-dependent Changes in Immediate Early Gene Labeling and Spine Density Following Abstinence from Contingent or Non-contingent Chocolate Pellet Delivery

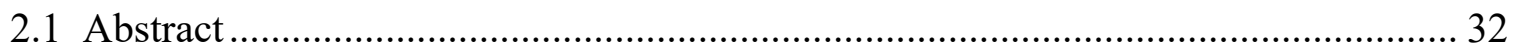

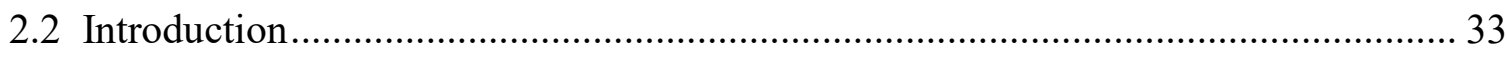

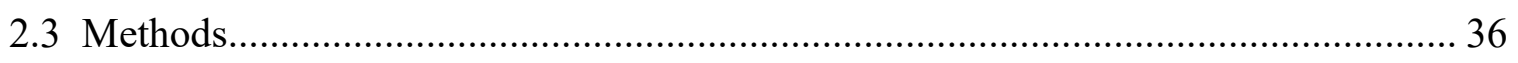

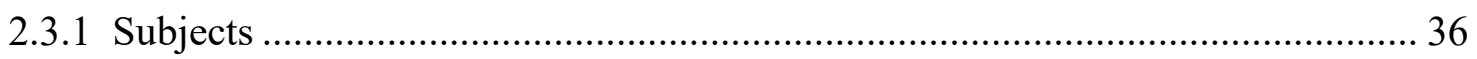

2.3.2 Operant acquisition and responding for cues ............................................ 36

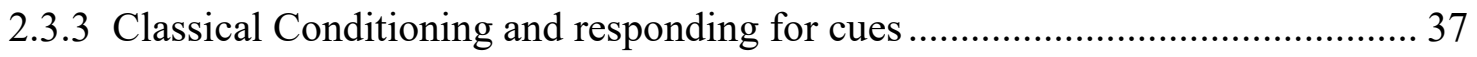

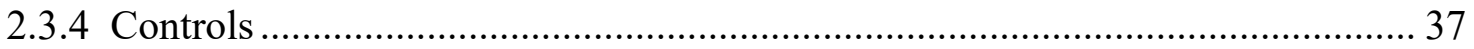

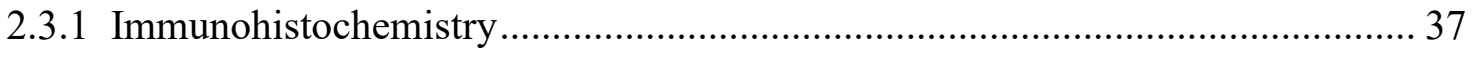

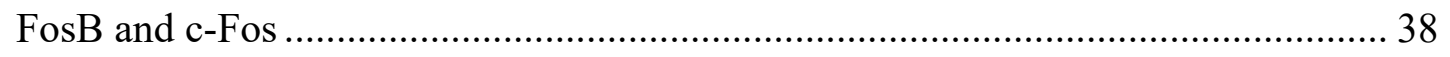

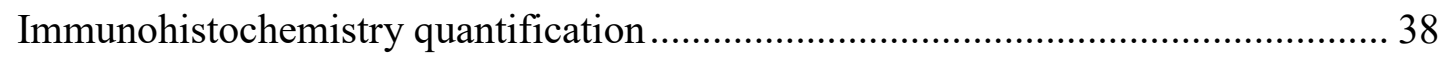

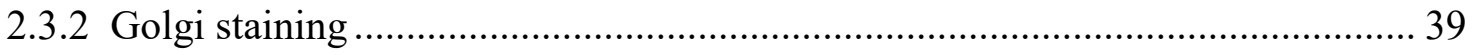

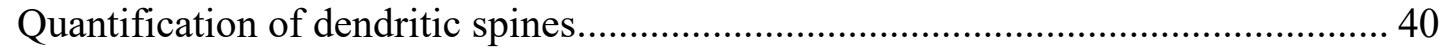

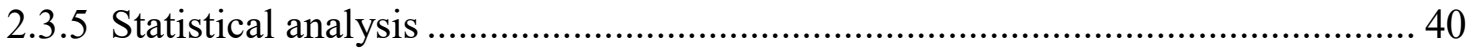

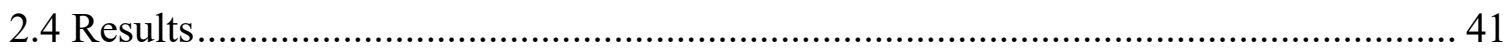

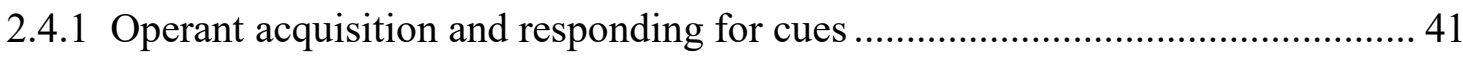

2.4.2 Classical conditioning and responding for cues .......................................... 41

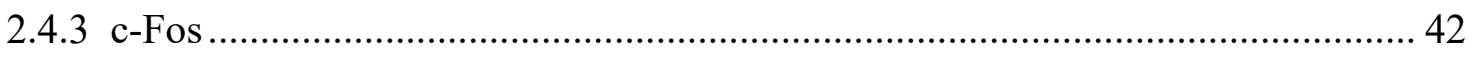

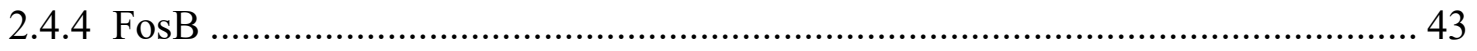

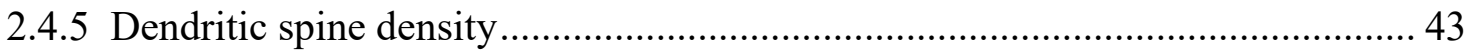

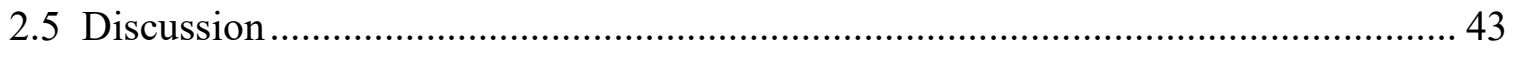

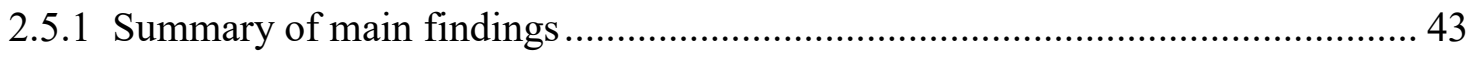

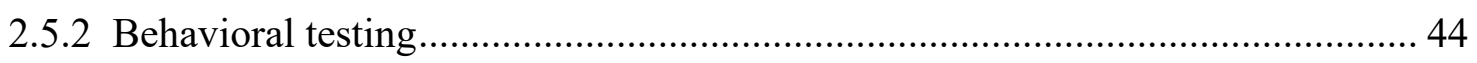

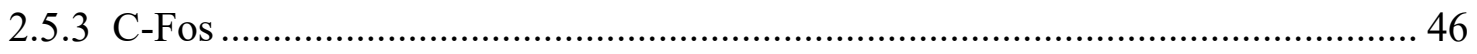

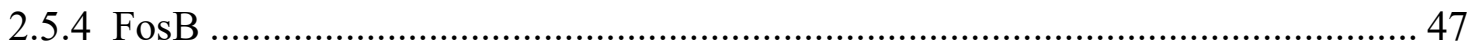

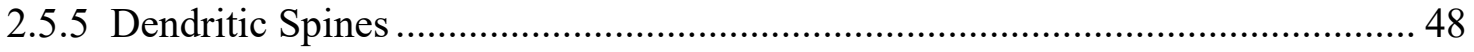

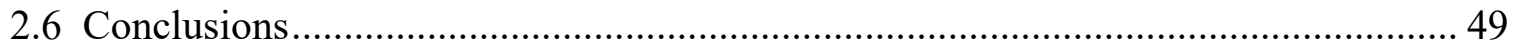

Chapter 3: Exploring Time-Dependent Changes in Conditioned Place Preference for Food Reward and Associated Changes in the Nucleus accumbens 


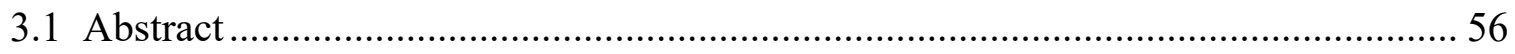

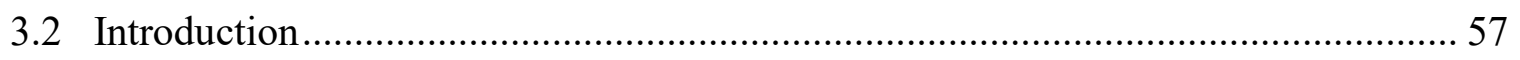

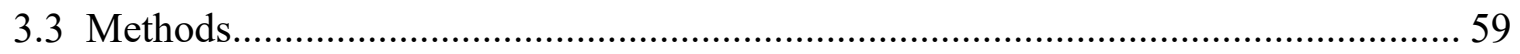

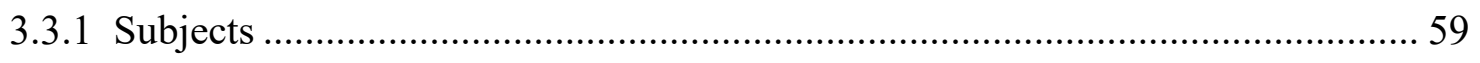

3.3.2 Conditioned place preference procedure ……................................................. 59

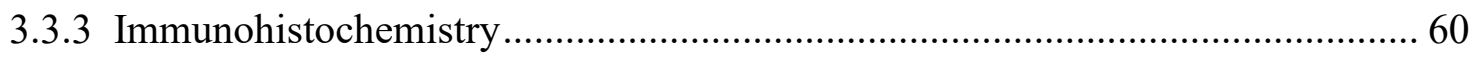

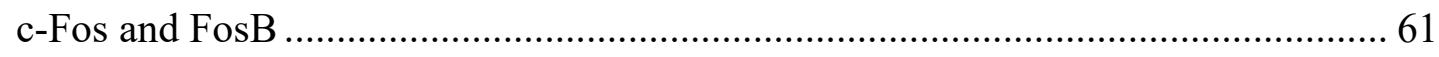

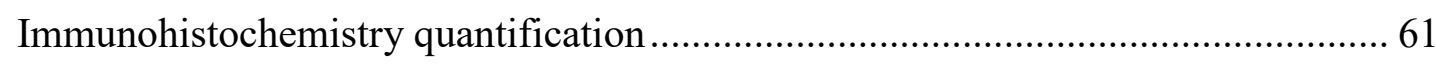

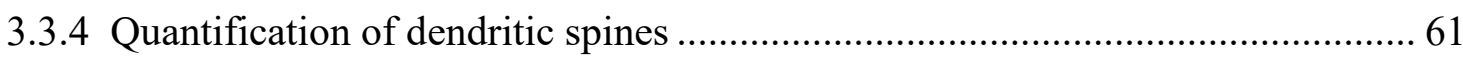

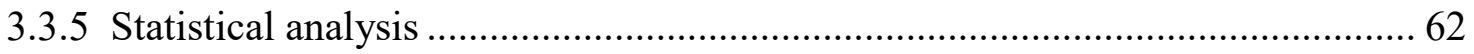

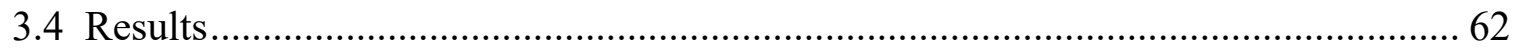

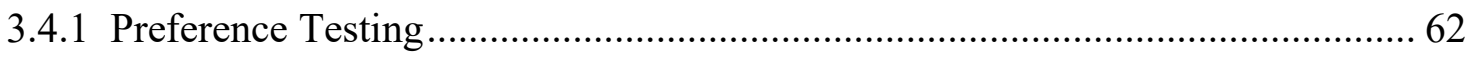

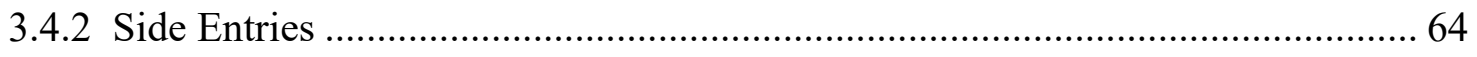

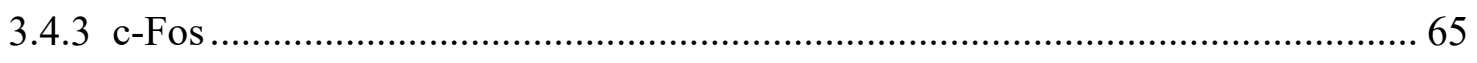

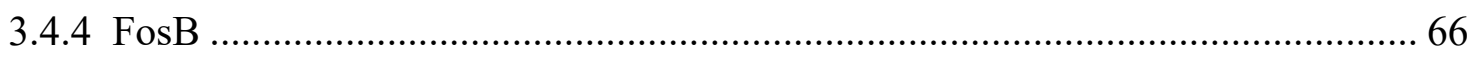

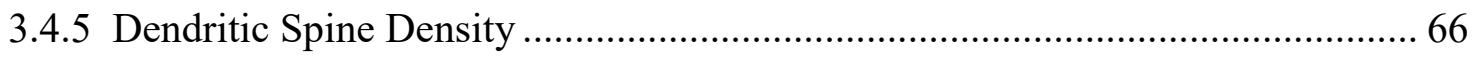

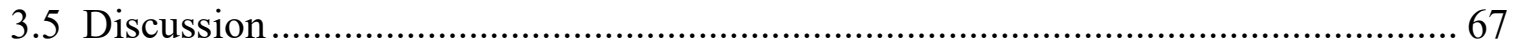

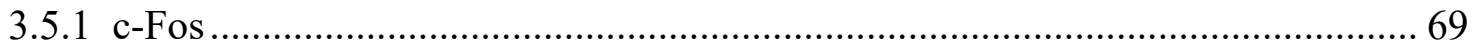

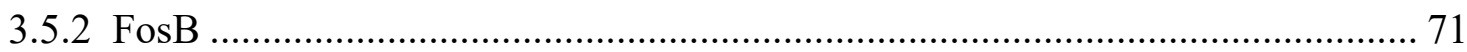

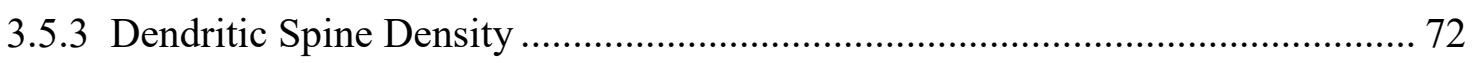

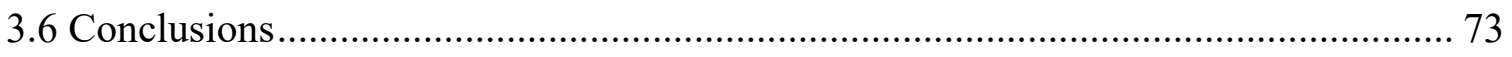

\section{Chapter 4: Time-dependent Changes in GluR1 Protein Expression} Following Abstinence From Food Rewards of Different Macronutrient Composition

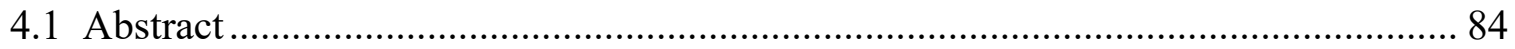

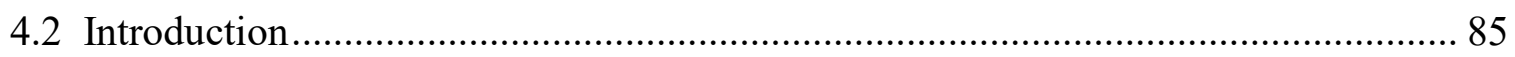

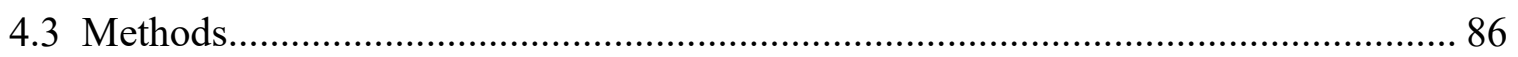

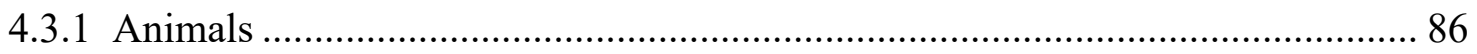

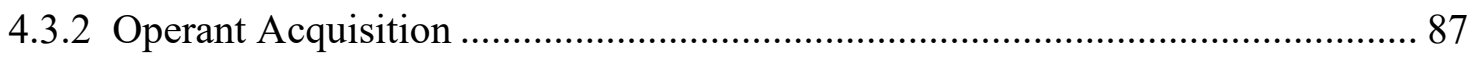

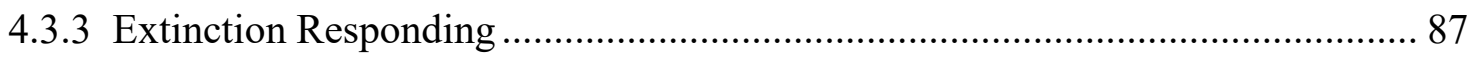

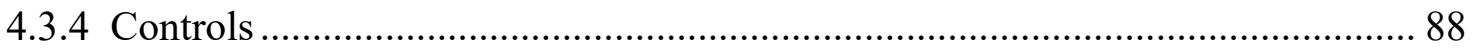

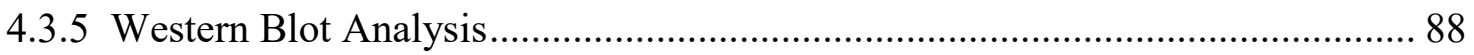

Protein Extraction and Quantification .................................................................. 88 


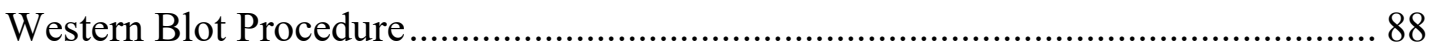

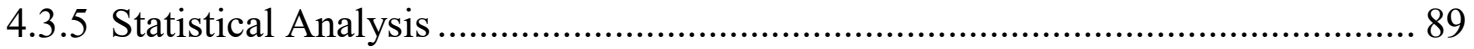

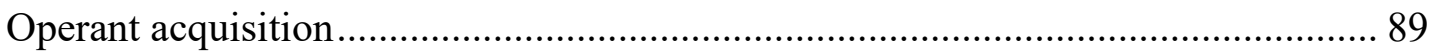

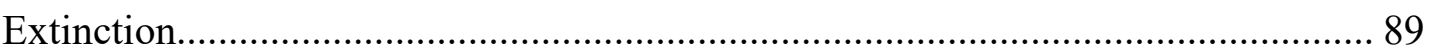

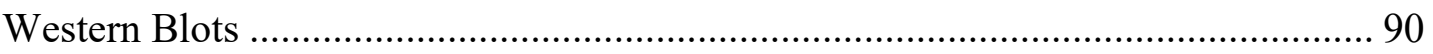

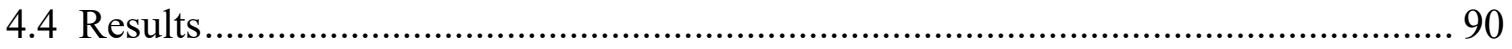

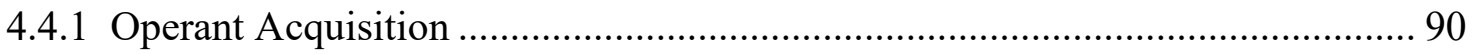

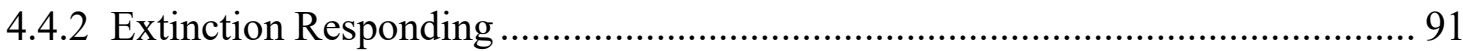

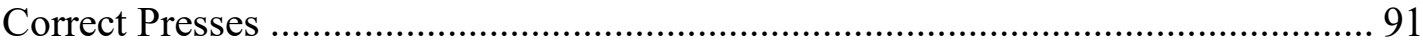

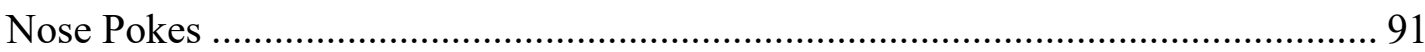

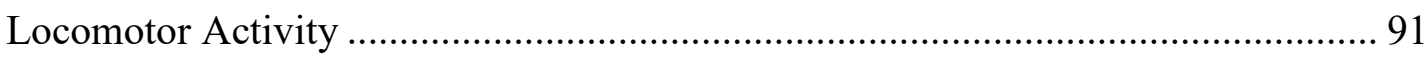

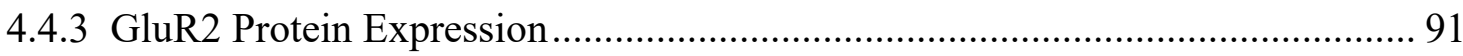

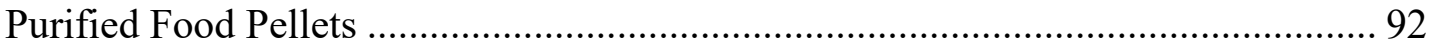

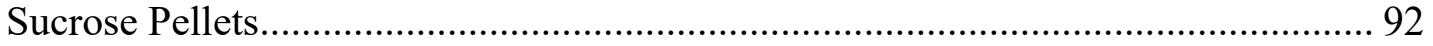

Chocolate Pellets ........................................................................................ 92

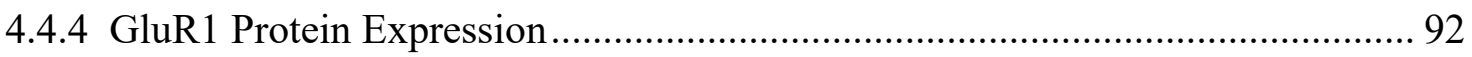

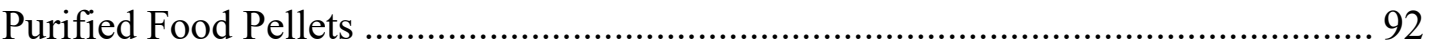

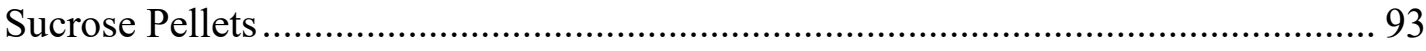

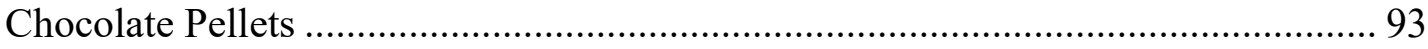

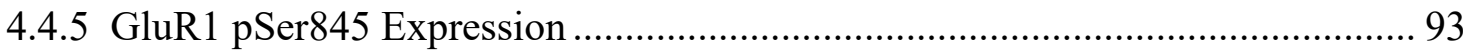

Purified Food Pellets ............................................................................................ 93

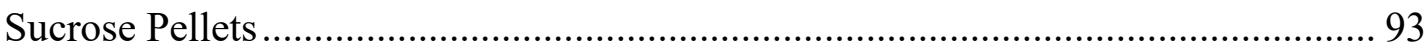

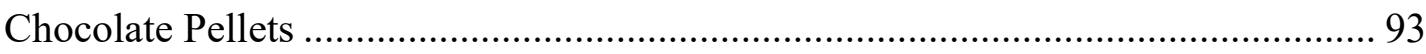

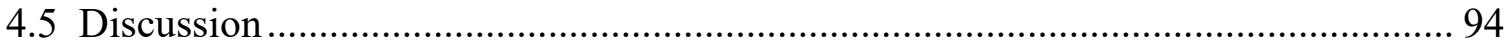

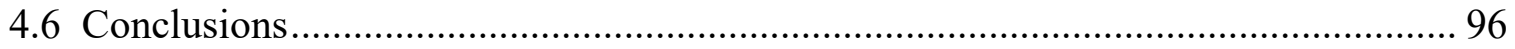

\section{Chapter 5: General Discussion}

5.1 Conclusions

\section{Chapter 6: Appendix A Copyright Documentation}

6.1 Permission from Frontiers in Behavioural Neuroscience ........................................ 108

6.2 Co-author Permission Statements ......................................................................... 110

Chapter 7: Appendix B Supervisor's Statement........................114

Chapter 8: Appendix C Western Blots.................................116 
References

\section{List of Figures}

Figure 2.1: Representative images of NAc regions........................50

Figure 2.2: Operant conditioning acquisition and testing....................51

Figure 2.3: Classical conditioning acquisition and testing....................52

Figure 2.4: Average c-Fos density...................................53

Figure 2.5: Average FosB density ....................................54

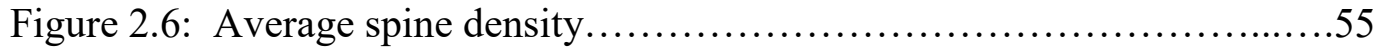

Figure 3.1: Representative image of NAc region and spines..................74

Figure 3.2: Preference ratio during CPP test............................ 75

Figure 3.3: Preference scores per 10-min test interval......................76

Figure 3.4: Side entries during CPP test.............................. 77

Figure 3.5: Average c-Fos density....................................78

Figure 3.6: Correlations between food and c-Fos labelling......................79

Figure 3.7: Average FosB density................................... 80

Figure 3.8: Average spine density NAcSh..............................81

Figure 3.9: Average spine density NAcC...............................82

Figure 3.10: Representative images of dendritic spines....................83

Figure 4.1: Operant acquisition...................................97

Figure 4.2: Operant extinction..................................... 98

Figure 4.3: GluR2 expression.....................................99

Figure 4.4: GluR1 expression.......................................... 100

Figure 4.5: GluR1 pSer845 expression................................101 


\section{List of Tables}

Table 3.1: Macronutrient composition of chocolate foods.........................60

Table 4.1: Nutritional profile of food pellets..........................87 


\section{Preface}

Following periods of abstinence from drugs of abuse and natural rewards, such as sucrose, time-dependent increases in substance-seeking behavior have been noted. This "incubation of craving" may be associated with subsequent relapse to substance use.

Food-seeking behavior has shown similarities to drug-seeking, with relapse from dieting similar to drugs of abuse, above $80 \%$. Palatable food craving may contribute to the vicious cycle of weight loss and weight gain. Understanding disordered eating behavior is important because comorbid diseases, such as diabetes and heart disease, account for a large majority of premature deaths. Individuals who suffer from disordered eating behavior are also more likely to suffer from comorbid drug use or have a history of drug use suggesting similar deficits in reward pathways. Understanding these reward pathways may provide insight into the rising obesity epidemic and eventually contribute to the development of treatments.

The nucleus accumbens (NAc) is part of the ventral striatum and is involved in the mediation of emotional and motivational processes. Alteration in neural activity within the NAc has been implicated in the development of neurological and psychiatric conditions including addiction. Drugs of abuse, such as cocaine, and palatable foods have been shown to alter dopamine activity within the NAc, which is associated with maladaptive behavior. In incubation of craving models, alterations in NAc plasticity has been shown to be time-dependent following periods of abstinence. For example, following 30 days of abstinence from cocaine, alterations in AMPA receptors have been noted. Time-dependent changes in food-seeking and associated NAc changes have also been shown differ depending on the type of administration during behavioral learning, such as contingent or non-contingent administration. The type of reward involved in incubation of craving models has also been shown to affect the magnitude of incubation effect following forced abstinence. The present thesis contains three manuscripts that investigate the potential for an incubation response on a behavioral and neural level following different methods of food administration (contingent and non-contingent) and food types of different flavor and macronutrient composition. These manuscripts provide structural and functional evidence that the type of behavioral model and food reward differentially affect food-seeking behavior and associated NAc changes.

Manuscript 1 (published in Frontiers in Behavioral Neuroscience) investigates incubation of food craving following abstinence from chocolate flavored pellets which were administered via operant (contingent) or classical conditioning (non-contingent). The NAc was examined for neuronal activation (c-Fos, FosB) and structural changes (spine density) following periods of forced abstinence from chocolate flavored pellets. Both methods of administration resulted in increased dendritic spine density compared to control animals. Dendritic spine density was associated with consistent food-seeking throughout the periods of forced abstinence. Classically conditioned animals displayed lower levels of NAc activation compared to both operant trained animals and food restricted controls. This manuscript provided evidence that different behavioral models may result in similar behavioral outcomes, but the neural correlates involved may differ. 
Manuscript 2 (to be submitted to Behavioural Brain Research) investigates the incubation of food craving following abstinence from chocolate flavored pellets or milk chocolate chips using a conditioned place preference (CPP) design. The NAc was examined for neuronal activation (c-Fos, FosB) and structural changes (spine density) following periods of forced abstinence from chocolate flavored pellets or milk chocolate chips. No incubation of craving response was noted for either food and only the chocolate pellet group displayed a place preference, which declined over the abstinence periods. Neuronal activation did not differ between food groups, but the chocolate pellet group differed from control at the 7-day abstinence period. Dendritic spine density differed between food groups at the 28-day abstinence period where the chocolate pellet group displayed significantly higher spine density compared to milk chocolate. The Structural changes were not associated with place preference behavior. Manuscript 2 provides evidence that foods of different macronutrient composition may differentially affect behavior and associated NAc changes.

Manuscript 3 (to be submitted to Neuroscience) investigates the potential incubation of craving response following operant conditioning with foods of different macronutrient composition and/or flavor (purified, sucrose, and chocolate sucrose pellets). Associated changes in AMPA receptor protein composition, GluR1, GluR1 pSer845, and GluR2, were investigated in the NAc. Only the purified group displayed an incubation of craving response over the abstinence periods, but the sucrose and chocolate groups displayed consistent responding across periods. GluR1 expression timedependently decreased in the purified and sucrose groups and was rapidly increase in response to cues previously paired with food reward in all food groups. Manuscript 3 shows that an incubation of craving response may not be directly associated with alterations in AMPA composition as the group that displayed an incubation response showed a similar pattern of expression as a group that did not. Building on Manuscript 2, Manuscript 3 also shows that foods of different macronutrient composition, that were utilized in the same behavioral conditioning, may not result in the same food-seeking behavior during abstinence.

\section{Contribution of Authors}

Manuscript 1 has three authors: the candidate, her supervisor, and an additional author, a summer student, who assisted in conducting some of the behavioral procedures and histology under the supervision of the candidate. Manuscript 2 has two authors: the candidate and her supervisor. Manuscript 3 has three authors: the candidate, her supervisor, and an honour's student who assisted in conducting the behavioral procedures. The conception and design of experiments was provided by the candidate whom conducted behavioural testing, histology, and analyzed data. The candidate drafted and revised the manuscripts and served as corresponding author for reviewer comments. The supervisor provided guidance in study design, analysis and interpretation. The supervisor edited all written text and provided approval for the submission and revision of manuscripts. 


\section{Chapter 1}

\section{Introduction}

\subsection{Overview}

\subsubsection{Addiction and Food}

Addiction, or substance use disorder (as defined by the DSM-V, 2013), is a broadly defined term that describes a loss of control over drug taking and a compulsion to take a drug even in the presence of adverse consequences (Koob \& Volkow, 2010; Kreek, LaForge, \& Butelman, 2002). Even after the physiological symptoms of withdrawal have subsided, individuals may experience a pathological preoccupation (craving) with their substance of choice, that subsequently leads to relapse (Kreek et al., 2002; Weiss, 2005). Craving is not unique to drug addiction as people have experienced food craving, particularly for high fat and high sugar foods, when they are trying to maintain a strict diet to lose weight. For example, chocolate, which contains a combination of ingredients, including, sugar, fat, and cocoa, is considered to be the most craved substance in the United States, where it is consumed in higher quantities compared to other countries (Nasser et al., 2011). Palatable food cravings may contribute to the vicious cycle of weight loss and weight gain in dieting individuals (Avena et al., 2008).

\subsubsection{Incubation of Craving}

In substance use disorder, craving may continue even after physiological withdrawal symptoms have subsided (Grimm, Shaham, \& Hope, 2002; Grimm et al., 2016; Lu, Grimm, Hope, \& Shaham, 2004; Pickens et al., 2011). In animal models, craving can be defined as an increase in responding for a substance-related cue, in the absence of that substance. Animal research has suggested the onset of craving may be delayed, and even enhanced, following a period of abstinence from a substance (Grimm et al., 2001, 2011a; Lu et al., 2004). Following a period of abstinence from cocaine, rats showed an increase in cue-induced responding on day 30 compared to day 1 of withdrawal (Aoyama et al., 2014; Li and Frantz, 2009; Lu et al., 2005). This enhanced and delayed craving following a period of abstinence that increases in a time-dependent manner is termed "incubation of craving" (Guillem et al., 2014; Lu et al., 2004; Pickens et al., 2011). Incubation of craving has been less extensively studied in relation to natural rewards but has been shown following liquid sucrose, (Grimm, Fyall, \& Osincup, 2005; Grimm, Manaois, Osincup, Wells, \& Buse, 2007), high fat (Darling et al., 2016; Dingess et al., 2017a), standard chow (Darling et al., 2016; Dingess et al., 2017a), and a preferred food pellet containing $12.7 \%$ fat and $66.7 \%$ carbohydrate (Krasnova et al., 2014c). 


\subsubsection{Food Craving}

Food and drugs have been shown to share similarities in their activation of reward circuitry, namely the nucleus accumbens (NAc) (Carr et al., 2000; Kelley and Berridge, 2002; McClung et al., 2004a; Olausson, 2006; Rogers and Smit, 2000; Wise, 2004), but there are few studies that have investigated the incubation of craving for palatable foods (Darling et al., 2016; Dingess et al., 2017a; Krasnova et al., 2014c) independent of liquid sucrose (Grimm et al., 2003, 2011a). Understanding the mechanisms of food craving has implications in combating the rising obesity epidemic by contributing to the development of appropriate behavioural and pharmacological treatments. In the past 50 years, the consumption of high fat foods has increased by $42 \%$ in North America (Davis and Carter, 2009). The food industry appears to have taken advantage of the natural desire for sugar and fat by increasing the daily "dose" of high fat and high sugar foods in meals (Davis and Carter, 2009). Since foods high in fat and sugar are widely consumed, foods high in these macronutrients are ideal to study food craving.

\subsection{Specific Aims}

The primary goals of this dissertation were:

1. To examine if forced abstinence from food reward results in a continued and/or delayed and amplified craving response (incubation of craving).

2. Determine if an incubation of craving response can be elicited by utilizing different behavioral designs (contingent vs non-contingent reward delivery).

3. Examine immediate early gene expression and dendritic spine changes in the NAc over periods of forced abstinence from food reward and the association of these markers with food-seeking behavior.

4. examine alterations in AMPA receptor protein expression in the NAc following forced abstinence from food reward

5. To examine if an incubation of craving response can be elicited by food rewards of different macronutrient composition or flavor and if this affects immediate early gene labelling, dendritic spine density, or AMPA receptor protein expression.

\subsection{Food Craving}

The concept of addiction and craving was described as early as the $18^{\text {th }}$ century in an attempt to explain the continued use of a substance even in the presence of adverse consequences (Drummond, 2001). Lettsom (1787) noted that alcohol "becomes as necessary as food and that neither threats nor persuasions are powerful enough to overcome the desire" (p.187) and Kerr (1889) referred to alcohol craving as "pathological depravity of the appetite centre" (p. 221) (Drummond, 2001). Although opium and alcohol were considered addictive, the first empirical data were not produced until the $19^{\text {th }}$ century (Crocq, 2007). A father of modern psychiatry, Emil Kraepelin, published the first psychometric data on the influence of tea and alcohol in the 1890's (Crocq, 2007). 
Kraeplin viewed alcoholism as a mental illness and stressed the importance of biological and genetic factors in the aetiology of mental disorders (Nathan et al., 2015). Kraepelin concluded that chronic alcoholism resulted in permanent cognitive decline as the result of brain lesions (Engstrom \& Kraepelin, 1990; Engstrom \& Kraepelin, 1991). By the 20 century, there were diagnostic classifications for addiction and research into its neurobiological correlates (Crocq, 2007).

Drugs are not the only substances considered to be addictive and in recent years, food addiction has been defined (Hebebrand et al., 2014). Randolph (1956) appears to be the one of the first to define the concept of food addiction (as cited in Hebebrand et al., 2014), indicating that highly palatable foods may have an addictive potential similar to drugs of abuse (Meule, 2015). "Food addiction" has been used in an effort to explain the growing prevalence of obesity (Ogden et al., 2014) and as an attempt to understand the psychological factors implicated in weight gain (Hebebrand et al., 2014). "Food addiction" is not classified in the DSM-V, but there have been attempts to compare compulsive overeating to the addiction cycle, such as binging, withdrawal, and craving (Meule and Gearhardt, 2014). Classifying what constitutes disordered food craving and overeating is an important step in identifying patterns that may lead to obesity and associated health effects.

\subsubsection{Importance of Studying Food Craving}

Food cravings may prevent successful weight loss by leading to overeating, and preventing weight loss and maintenance (Forman et al., 2007). Obesity results when the number of calories consumed is greater than the number of calories expended (Barry et al., 2010). It is estimated that $23-25 \%$ of Canadians are obese (BMI 30+) and this number does not include people who are overweight (BMI 25-30) (Canadian Institute for Health Information, 2011). Obesity accounts for at least $9 \%$ of all deaths in adults aged 20-64 due to comorbid diseases such as diabetes and heart disease (Canadian Institute for Health Information, 2011). Sedentary lifestyle and the consumption of calorie dense foods contribute to the calorie imbalance (Barry et al., 2010). Relapse rates within a year of diet initiation are similar to those seen with drugs of abuse at approximately $80 \%$ (Brandon et al., 2007).

Individuals suffering from disordered eating are more likely to suffer from comorbid drug use or have a history of drug use (Barry et al., 2010). For example, excessive alcohol use, but not moderate use, has been associated with obesity in some individuals, and in this group, obesity is more prevalent in women (Traversy and Chaput, 2015). A previous history of alcohol abuse is also reported in both normal and overweight women that experience regular food craving (Gendall et al., 1997). Severe food cravings have also been shown to be a comorbid with heroin use disorder in men (Canan et al., 2017). This highlights the potential for similar reward processing in the brain between food cravings and drug use (García-García et al., 2014).

\subsubsection{Similarities Between Drug and Food Craving}


Addiction is a broadly defined term that encompasses a loss of control over drug taking and a compulsion to take a drug even in the presence of adverse consequences (Koob, 2000; Kreek et al., 2002). The Diagnostic and Statistical Manual of Mental Disorders (DSM) classifies addiction under substance-related disorders (DSM-V, 2013). The Substance-related disorders include 10 classes of drugs: alcohol, caffeine, cannabis, hallucinogens, inhalants, opioids, sedatives, hypnotics, anxiolytics, stimulants, tobacco, and other unknown substances (DSM-V, 2013). Behavioural addictions other than pathological gambling are not currently classified in the DSM-V as there is insufficient peer-reviewed evidence for such addictions (DSM-V, 2013).

According to the DSM-V, substance use disorder results in a cluster of cognitive, behavioural, and physiological symptoms whereby an individual continues using a substance despite significant health and interpersonal problems (DSM-V, 2013). In order for an individual to be diagnosed with a substance use disorder, they must meet criteria set out by the DSM-V (DSM-V, 2013). Criterion A consists of 11 groupings that encompass impaired control, social impairment, risky use, and pharmacological criteria. It is suggested that a mild substance use disorder has the presence of two or three criteria symptoms, moderate will have four or five symptoms, and severe will have six or more symptoms. The severity of a substance use disorder can change across time and is determined by reduction/increase in the frequency of substance use, clinical observations, and biological testing (DSM-V, 2013). The compulsivity of drug use makes it difficult for an individual to remain drug free for extensive periods of time, as drug use tends to following a cyclical pattern where there are periods of binging, withdrawal, and craving (Joffe, Grueter, \& Grueter, 2015; Koob \& Volkow, 2010).

Compulsive overeating, which can lead to obesity, may share similar characteristics to substance use disorders (Meule and Gearhardt, 2014). The Yale Food Addiction Scale (YFAS) was developed in an attempt to categorize food addiction based on the DSM-IV criteria for substance dependence (Meule and Gearhardt, 2014). Examples of criteria for food addiction in the YFAS include (Meule and Gearhardt, 2014):

- A persistent desire or unsuccessful efforts to cut down or control eating.

- Continued eating despite physical or psychological problems.

- Consumption of large amounts of food over a longer period of time than intended.

- Spending much time obtaining food.

- Giving up important activities.

A comparison between aspects of "food addiction" and the DSM-V will continue throughout this section, in order to highlight the similarities and differences between food and drug addiction, in terms of binging, withdrawal, and craving.

Binge/Intoxication

Substances that are associated with addiction tend to be both rewarding and reinforcing, meaning they are interpreted as having positive valence which increases the 
likelihood that ingesting the substance will be repeated (Hyman \& Malenka, 2001). Initial ingestion of a substance can be impulsive and motivated by positive reinforcement where the expectation of drug effects may influence the rewarding properties (Joffe et al., 2015; Koob \& Volkow, 2010). Initial drug experimentation can also result from conforming to social groups (peer pressure) where the reinforcing effects are subsequently discovered (Koob \& Volkow, 2010). Substance abuse may also result from the therapeutic properties of analgesics used for pain management (Koob \& Volkow, 2010). Since food is considered necessary for survival, the beginnings of compulsive overeating may be less clear than drug use. However, individuals considered to have a "food addiction" show similar bingeing behaviour to those who abuse drugs (Davis and Carter, 2009). When an individual relapses while on a diet, they are likely to experience a binging episode when they consume more calories, and calorie dense foods, than individuals without food addiction (Davis and Carter, 2009). A binge eating episode is not driven by hunger or metabolic need, and involves rapid and excessive consumption of food (Mathes et al., 2009).

Repeated drug administration may result in homeostatic adaptations within the brain leading to tolerance (Hyman et al., 2006). If an individual develops drug tolerance following repeated drug administration, they will require an increased drug dose to get the desired rewarding effects (Hyman \& Malenka, 2001). For example: individuals taking cocaine may require a larger dose in order to get the same pleasurable effects (e.g. euphoria) (Hyman \& Malenka, 2001). A similar phenomenon has been observed in individuals who overeat. They require an increased amount of food to achieve a desired effect, as continuing to eat the same amount of food is not satisfying (Meule and Gearhardt, 2014). When an individual repeatedly uses a substance, it may result in changes within the brain that make it difficult to control their behaviour, and subsequently, result in compulsive drug use or overeating despite negative consequences (Hyman \& Malenka, 2001; Joffe et al., 2015).

\section{Withdrawal/Negative Affect}

Cessation of a substance following heavy or prolonged use can result in withdrawal which leads to aversive physical and behavioural symptoms (DSM-V, 2013). Withdrawal symptoms can also result from exposure to environmental stimuli that were previously associated with drug use (Piper, 2015). All drugs of abuse have the potential to result in withdrawal symptoms, which can range from acute intense physiological symptoms (nausea, vomiting) to dysphoria, irritability, emotional distress, and sleep disturbances (Koob \& Volkow, 2010). The acute physiological symptoms can be so severe that if not treated, death can result (Koob \& Volkow, 2010). In order to reduce withdrawal symptoms, an individual may self-administer their preferred substance (DSM-V, 2013). This negative reinforcement (removal of withdrawal symptoms by taking the drug) may be responsible for the development of drug dependence following the positive effects of initial drug use (Koob, 2000). While withdrawal symptoms are plausible with food addiction they can be difficult to distinguish from the energy deficit that results from hunger, which can result in agitation and anxiety (Meule and Gearhardt, 2014). 


\section{Preoccupation/Craving}

Addiction is often characterized by repeated bouts of relapse even after extended periods of abstinence and after physiological withdrawal symptoms have subsided (Langleben et al., 2008; Lubman et al., 2004). Relapse is usually defined as any drug use following a period of sustained abstinence (Brandon et al., 2007). Relapse rates for drug use range from $75 \%-92 \%$ and for food addiction, relapse rates are consistently reported to be at $\sim 80 \%$ (Brandon et al., 2007). Craving is a phenomenon that describes an intense desire to self-administer drugs and may result from increased motivation towards a substance (Avena et al., 2008). Craving following periods of abstinence from drugs (Bossert, Ghitza, Lu, Epstein, \& Shaham, 2005; Koob, 2000; Newton, De La Garza, Kalechstein, Tziortzis, \& Jacobsen, 2009; Sinha, 2013), and food (Gendall et al., 1997; Meule and Kübler, 2012), may result in relapse to drug use, binge eating, and unhealthy dietary habits in susceptible individuals

Craving is a similar phenomenon reported in both drug addiction and disordered eating (Meule and Kübler, 2012). People who are trying to maintain a strict diet describe food craving, particularly for high fat and high sugar foods. This craving is argued to be a main contributing factor to the vicious cycle of weight loss and weight gain (Avena et al., 2008). Individuals that measure high on the YFAS have higher self-reports of food craving compared to individuals that have lower scores (Davis et al., 2011; Meule and Kübler, 2012). These individuals may experience craving for palatable food (e.g. chocolate and ice cream) in a similar manner that drug addicts crave drugs (Meule and Gearhardt, 2014; Meule and Kübler, 2012; Rogers and Smit, 2000). Craving intensity may differ depending on the food, craving for chocolate has been shown to be independent from craving for sweet taste, suggesting chocolate addiction may be a phenomenon on its own (Hill and Heaton-Brown, 1994). It has even been questioned if chocolate should be considered a food or a drug (Bruinsma and Taren, 1999).

Craving can result from a sensitivity to environmental cues previously associated with drug use (such as the location of drug intoxication), resulting in relapse to drug-taking (Koob \& Volkow, 2010). Drug relapse may result in an individual taking a substance in larger amounts and for a longer period of time than was intended (DSM-V, 2013). Craving can result in risky drug use where an individual will use a substance in a situation that could be physically hazardous (DSM-V, 2013). This preoccupation/craving with a drug may result in social impairment and interpersonal problems along with a failure to fulfill major roles at work, school, or home (DSM-V, 2013). Unlike drug addicts, individuals who overeat are usually not in acute danger (chronic health problems may exist), but may suffer from interpersonal problems, such as social isolation, social insecurity, and hostility (Meule and Gearhardt, 2014). Understanding craving, and its underlying neural mechanisms, may be central for the development of successful treatments for addiction (drug and food), and preventing relapse following periods of sustained abstinence.

\subsection{Nucleus Accumbens}


The term nucleus accumbens (NAc) was first used in 1904 by Ziehen to describe the round flattened structure within the striatum (Ziehen, 1904). The NAc is part of the ventral striatum, which plays a role in mediating emotional and motivational processes (Salgado \& Kaplitt, 2015). The NAc has been implicated in several neurological and psychiatric disorders, including: Parkinson's disease (Mavridis et al., 2011), depression (Goff et al., 2013; Shirayama and Chaki, 2006), and addiction (Chaudhri, Sahuque, Schairer, \& Janak, 2010; Chiara et al., 2004; Grimm, Harkness, et al., 2011; Wolf \& Tseng, 2012). Food has also been shown to activate the NAc in a similar manner to drugs of abuse (Bassareo et al., 2015a; Bassareo and Di Chiara, 1999; Peng et al., 2011a; Velázquez-Sánchez et al., 2014b; Wise, 2006), both of which contribute to long-term changes in gene expression (Caster and Kuhn, 2009; Kelz and Nestler, 2000; Nestler et al., 2001) that is associated with susceptibility to craving.

\subsubsection{Afferents/Efferents}

The NAc is located anterior to the superior border of the anterior commissure, lies parallel to the midline, and extends dorsolatterally into the putamen and dorsomedially into the caudate nucleus (Salgado and Kaplitt, 2015). The NAc is comprised of two main subregions, the core (NAcC) and shell (NAcSh), which have different afferent/efferent connections, neurochemistry, and functionality (Usuda et al., 1998; Záborszky et al., 1985). Distinctions between the NAcC and NAcSh subregions have been demonstrated using immunohistochemical reactions of calbindin and substance $\mathrm{P}$ in addition to DA innervation (Bardo and Hammer, 1991; Deutch et al., 1988; Hussain and Totterdell, 1994; Zahm and Brog, 1992). Minor cell morphology differences exist between the NAc subregions with neurons in the NAcSh having fewer dendritic branch segments and a lower spine density than the NAcC (Meredith et al., 1992).

The NAc receives afferent DA projections primarily from the VTA and numerous glutamate projections from the PFC, amygdala, hippocampus, and thalamus (Wise, 1996). The dorsomedial NAcSh has been shown to receive afferent projections from the ventral prelimbic cortex, anterior part of the paraventricular thalamic nucleus, and the ventral subiculum, while the ventromedial shell receives inputs from the infralimbic cortex, the anterior part of the paraventricular thalamic nucleus, caudal magnocellular part of the basal amygdaloid nucleus, the caudal part of the accessory basal amygdaloid nucleus, and the dorsal to ventral subiculum (Groenewegen et al., 1987; Wright et al., 1996; Wright and Groenewegen, 1996). There are afferent projections to the ventrolateral NAcSh from the rostral magnocellular part of the basal amygdaloid nucleus whereas the NAcC receives inputs from the prelimbic cortex, anterior part of the paraventricular thalamic nucleus, rostral and caudal magnocellular parts and the parvicellular part of the basal amygdaloid nucleus, and the medial part of the accessory basal amygdaloid nucleus (Groenewegen et al., 1987; Wright et al., 1996; Wright and Groenewegen, 1996).

A study by Usuda and colleagues (1998) determined that efferent projections from the NAc differ for the NAcC and NAcSh, which was elucidated using a biotinylated dextran amine as an anterograde tracer (Usuda et al., 1998). In the rostral NAc the dorsomedial NAcSh was shown to mainly project to the dorsomedial ventral pallidum, 
lateral hypothalamus, and substantia nigra pars compacta, whereas the ventromedial NAcSh projected to the ventromedial ventral pallidum, lateral preoptic area, lateral hypothalamus, and ventral tegmental area (Usuda et al., 1998). In the caudal NAc, the NAcSh projected to the dorsomedial ventral pallidum, lateral hypothalamus, and ventral tegmental area whereas the ventromedial shell projected to the ventromedial ventral pallidum, substantia innominate, ventral tegmental area, substantia nigra pars compacta, and retrorubral area (Usuda et al., 1998). In the rostral NAc, the dorsal NAcC was shown to project to the caudate putamen, dorsolateral ventral pallidum, globus pallidus, lateral hypothalamus, and substantia nigra pars reticulate. In the caudal NAc, the dorsomedial NAcC projected to the dorsal ventral pallidum, lateral hypothalamus, substantia nigra pars compacta, and substantia nigra pars reticulate, whereas the ventral and lateral NAcC had projections to the dorsolateral ventral pallidum, globus pallidus, and substantia nigra pars compacta (Usuda et al., 1998).

\subsubsection{Dopamine}

DA is a catecholamine neurotransmitter that is synthesized from the amino acid tyrosine (Beaulieu \& Gainetdinov, 2011). There are four main DA pathways in the brain including the nigrostriatal, mesolimbic, mesocortical, and tuberoinfundibular pathways (Beaulieu \& Gainetdinov, 2011). The nigrostriatal pathway consists of projections from the SN to the striatum as well as cortical and limbic regions (Björklund and Dunnett, 2007). This pathway is involved in the control of voluntary movement and goal-directed behaviours (Baik, 2013). The mesolimbic pathway consists of neurons that project from the VTA to the NAc (ventral striatum) as well as the ventral medial head of the caudate putamen (Björklund and Dunnett, 2007). This pathway plays a role in motivation and reward (Baik, 2013). The mesocortical pathway projects primarily to the frontal cortex and also plays a role in motivation and reward (Baik, 2013). The tubero-infundibular pathways consists of cells in the arcuate nucleus and periventricular nucleus of the hypothalamus, which project to the pituitary and is involved in the synthesis and release of prolactin (Baik, 2013; Björklund \& Dunnett, 2007). The primary focus of this prospectus is on the mesolimbic DA pathway.

\section{Dopamine Receptors}

DA receptors are expressed in both the central and peripheral nervous system and have a high density within the mesocorticolimbic DA system (Beaulieu \& Gainetdinov, 2011). DA receptors can be divided into two types: D1 class receptors (D1 and D5) are coupled to the $\mathrm{G}_{\mathrm{s}}$ protein which can activate adenylate cyclase (AC) and subsequently increase cAMP, and D2 class receptors (D2, D3, and D4) are coupled to the $\mathrm{G}_{\mathrm{i}}$ protein which inhibits AC, inhibiting cAMP production (Beaulieu \& Gainetdinov, 2011). D2 receptors are the primary DA autoreceptor and are involved in the presynaptic regulation of DA firing rate, synthesis, and release (Beaulieu and Gainetdinov, 2011). D1 and D2 receptors are found on GABAergic MSN's within the NAc and a limited number of D3 receptors are found within the NAcSh on MSN (Beaulieu \& Gainetdinov, 2011).

DA receptor Signalling 
DA signalling is complex as activation of one receptor can begin a cascade of events that leads to regulation of numerous proteins and kinases, including cyclic adenosine monophosphate (cAMP) and protein kinase A (PKA) (Beaulieu \& Gainetdinov, 2011). When a D1 and/or D2 receptor are inactive, the $\alpha$-subunit is bound to GDP and a $\beta \gamma$-complex, which forms a trimeric protein complex (Beaulieu \& Gainetdinov, 2011). When an agonist is bound to the D1 or D2 receptor, it results in GDP release and GTP binding to the $\alpha$-subunit, resulting in the dissociation of the $\alpha$ subunit and the $\beta \gamma$-complex (Beaulieu \& Gainetdinov, 2011). When the D2 receptor is activated, the $\mathrm{G} \alpha$ subunit inhibits the production of the second messenger cAMP, whereas the D1 receptor stimulates the production of cAMP, and the separated $\beta \gamma$ complex can engage in separate signalling activities such as activation of G-proteinregulated inwardly rectifying potassium channels (GIRK) (Beaulieu \& Gainetdinov, 2011). Activation of the cAMP pathway has been implicated in the induction of immediate early genes such as c-Fos, which is used as a measure of neuronal activity (Berretta et al., 2005; Renthal et al., 2008; Semba et al., 1996), and DeltaFosB, which is considered a marker of addiction, that can persist for weeks-months after cessation of a substance (Larson et al., 2010). Cyclic AMP and DeltaFosB are associated with the PKA pathway, which may be involved in behavioural sensitization that is associated with drug use (Chen et al., 2009).

\subsubsection{Glutamate}

Glutamate is the primary excitatory neurotransmitter within the central nervous system (Sheng and Kim, 2002). The majority of glutamatergic synapses within the brain are found on pyramidal cells within the cortex and hippocampus (Van den Oever, 2012). Neurological disorders such as epilepsy, neurodegenerative conditions, and stroke have been linked to aberrant ionotropic glutamate receptor activity: (Stawski et al., 2010). Glutamate within the NAc may play a role in addiction, as GABAergic MSNs receive glutamatergic inputs from the PFC (van Huijstee and Mansvelder, 2015). When these inputs are activated, they may play a role in craving by altering glutamate receptor composition (Wolf and Tseng, 2012).

\section{Ionotropic Glutamate Receptors}

Ionotropic glutamate receptors are ligand-gated, tetrameric, cation channels that mediate a fast response to the glutamate neurotransmitter (Stawski et al., 2010). There are three types of ionotropic glutamate receptors: N-methyl-D-aspartate (NMDA), kainite, and alpha-amino-hydroxy-5-methyl-4-isoxazolepropionic acid (AMPA) (Stawski et al., 2010). The NMDA receptor family consists of GluN1, GluN2A-Glun2D, GluN3A-GluN3B, which are heterotetrameric and contain subunits that can bind to glycine or d-serine (GluN1 and GluN3), and a GluN2 subunit that is activated by glutamate (Stawski et al., 2010). NMDA receptors are unique in that they are inactive at resting membrane potential due to a voltage-dependent block of the ion channel by a magnesium ion, which requires post synaptic excitation and glutamate binding to be expelled (Mayer et al., 1984; Nicoll and Malenka, 1999; Nowak et al., 1984). Kainate receptors consist of GluK1-GluK5 and are either homo- or heteromers (Stawski et al., 
2010). Kainate receptors exist both pre- and post-synaptically and appear to be involved in presynaptic plasticity (Stawski et al., 2010). NMDA, kainate, and CP-AMPA receptors (described below) are permeable to calcium, which can trigger intracellular signalling cascades, such as activation of CaMKII, affecting trafficking of receptor subunits (Mayer et al., 1984; Nicoll and Malenka, 1999; Nowak et al., 1984).

AMPA receptors are expressed throughout the central nervous system and are involved in fast synaptic transmission (Stawski et al., 2010). AMPA receptors consist of GluA1-GluA4 and are heterotetrameric or homotetrameric depending on their composition. In the VTA and NAc, AMPA receptors are thought to be heterotetrameric complexes consisting of GluR2/3 and GluR1 subunits (Van den Oever, 2012). AMPA receptors that lack the GluR2 subunit can be permeable to calcium (CP-AMPA), contributing to long-term synaptic changes (Malinow and Malenka, 2002; Van den Oever, 2012). Changes in AMPA receptor protein composition and density at postsynaptic synapses, which can affect intracellular signalling, represent a form of synaptic plasticity, which will be discussed in detail later (Stawski et al., 2010). Homomeric AMPA receptors are suggested to be the result of plasticity changes at synapses, resulting in GluR1 subunit insertion and the emergence of calcium permeable AMPA receptors (Van den Oever, 2012).

AMPA receptor signalling

AMPA receptors are responsible for fast excitatory neurotransmission within the NAc (Hanley, 2014). When glutamate, or an agonist, binds with an AMPA receptor, the result will be a conformational shift that opens a pore allowing the influx of sodium, potassium, and sometimes calcium into the neuron (Willard and Koochekpour, 2013). Whether calcium enters the cell depends on the type of AMPA receptor that is activated (Willard and Koochekpour, 2013). The majority of AMPA receptors are calcium impermeable (CI)-AMPA receptors, which maintain low $\mathrm{Ca} 2+$ concentrations in the neuron under basal conditions. CP-AMPA receptors are permeable to $\mathrm{CA} 2+$ and are involved in the regulation of CA2+ signalling pathways, which can lead to synaptic changes (Hanley, 2014). AMPA receptors can also interact with synaptic proteins via the cytoplasmic C-terminus. This cytoplasmic interaction can play a role in membrane distribution and trafficking of AMPA receptors (Willard and Koochekpour, 2013).

\subsubsection{Craving}

The hallmark action of drugs of abuse is an increase in extracellular DA within the NAc, which appears to be responsible for the reinforcing effects of addictive substances (Hyman et al., 2006; Wise, 2006). Natural rewards, such as palatable food, have also been shown to increase DA levels and human imaging studies have revealed increased activation in the striatum in response to both food and drugs (Breiter et al., 1997; Small et al., 2003). This DA release, may be key in the control of motivated behaviours (Baik, 2013; Baik, 2013) and play a role in reward related learning and shaping the craving for future reward-related behaviours (Hyman, 2005). For example: Robinson and Berridge have described an incentive-sensitization view of addiction, which suggests that DA release is associated with assigning incentive salience to 
rewarding substances and cues, triggering a state of wanting for the substance (Robinson $\&$ Berridge, 2000). Hence, craving can be triggered by an increase of DA activity in response to cues previously associated with palatable foods or drugs (Johnson \& Kenny, 2010; Stice, Yokum, Blum, \& Bohon, 2010; Stice, Spoor, Bohon, Veldhuizen, \& Small, 2008; Stoeckel et al., 2008).

Lesion, pharmacological, transgenic, and microdialysis studies have been used to determine that the rewarding properties of drugs of abuse and palatable foods rely on changes in DA activity within the NAc (Baik, 2013; Hyman, 2005a; Wang, Volkow, Thanos, Panayotis, \& Fowler, 2004; Wise \& Rompre, 1989). Both food and drugs can increase DA release (Wise, 2006), but the effect of initial DA release on subsequent drug and food intake has been shown to be different (Wise \& Rompre, 1989). When the mesolimbic DA pathway is lesioned, the rewarding effect of food is not hindered, whereas lesions reduce the rewarding aspects of drugs (Wise \& Rompre, 1989). These changes in DA have also been shown to differ between the NAcC and NAcSh subregions. The NAcC and NAcSh have been shown to have different roles in the acquisition of food and drug seeking behaviours (Chiara, 2002). The NAcSh is suggested to play a role in incentive arousal and lesions to the NAcSh prevent conditioned reinforcement for amphetamine whereas lesions to the NAcC do not (Parkinson et al., 1999). The NAcSh lesions have also been shown to produce locomotor hypoactivity whereas lesions to the NAcC produced hyperactivity and enhanced the stimulating effects of amphetamine (Parkinson et al., 1999). Differences in behavioural output resulting from stimulation or inactivation of these regions is plausible, as the NAcC and NAcSh have different afferent and efferent projections (Salgado and Kaplitt, 2015).

DA antagonism, particularly at the D1 receptor, has been shown to affect responding for cocaine and cocaine induced hyperlocomotor activity (Britton et al., 1991; Cabib, Castellano, Cestari, Filibeck, \& Puglisi-Allegra, 1991; Caine \& Koob, 1994; Wise, 2006). D1 and D2 receptor antagonists have been shown to dose dependently decrease cocaine self-administration when the drugs are injected directly into the NAc (Britton et al., 1991; Caine \& Koob, 1994). Administration of the D1 receptor antagonist $\mathrm{SCH} 23390$ has been shown to attenuate increased locomotor activity following acute cocaine administration, whereas D2 receptor antagonism with haloperidol does not have an effect (Cabib et al., 1991). Decreased cocaine self-administration has also been shown in D1 receptor knock-out mice (Barak Caine et al., 2007). DA antagonism has also been shown to dose-dependently decrease responding for food, but the rewarding aspects of food are not impacted (Wise, 2006). In this instance, animals will eventually reach normal performance (e.g. bar pressing), but it may require more trials (Wise, 2006). This may be the result of DA antagonism on locomotor activity, which could be independent from the rewarding aspects of food.

Reductions in D2 receptor density within the mesolimbic DA pathway have been associated with craving for both drugs and food (Huang et al., 2006; Johnson \& Kenny, 2010; Wang, Nora D. Volkow, Panayotis K. Thanos, \& Joanna S. Fowler, 2009). The consumption of high fat food has been shown to result in decreased D2 receptor density (Wang et al., 2009). These reductions in DA receptor density (D2) are similar when comparing drug addicts and people struggling with obesity (Wang et al., 2004). In 
rodents, high-fat diets have been shown to result in DA receptor sensitization as evidenced by increased sensitivity to DA agonists such as, cocaine, amphetamine, and quinpirole (Huang et al., 2006). A lower D2 density has been associated with increased responsiveness to environmental cues previously associated with addictive substances, possibly leading to craving (Johnson \& Kenny, 2010; Wang et al., 2009). For example: rats with reduced D2 density have been demonstrated to be hyper responsive to food cues, suggesting a craving response, and a risk for future weight gain (Johnson and Kenny, 2010).

Microdyalisis studies have revealed that the magnitude of DA release into the NAcC and NAcSh depends on the novelty of a substance (Chiara, 2002). When presented with a novel appetitive taste, such as chocolate, NAcSh DA rapidly increases but DA release in the NAcSh is unaffected by aversive substances (Chiara, 2002). In the $\mathrm{NAcC}$, novel taste stimuli increases DA, regardless of whether the stimuli was appetitive or aversive, (Chiara, 2002). After a single exposure to chocolate, but not drugs of abuse, DA transmission within the NAcSh habituates, but DA transmission does not habituate in the NAcC (Chiara, 2002). However, food restriction, even to a mild extent, can prevent DA habituation in response to palatable foods within the NAcSh (Chiara, 2002).

Elevation of DA, within the NAc, has been thought to be a primary mediator in drug addiction, but AMPA receptor activity may play an important role in the reinstatement of drug-seeking behaviour (Cornish and Kalivas, 2000). Injection of AMPA into the NAc results in reinstatement of cocaine-seeking behaviour; whereas, injection of the AMPA receptor antagonist CNQX prevents cocaine seeking (Cornish and Kalivas, 2000). The reinstatement of drug-seeking may be related to changes in AMPA receptor expression that result following periods of drug self-administration (Conrad et al., 2008; Li \& Wolf, 2011; Loweth, Tseng, \& Wolf, 2014; Terrier, Luscher, \& Pascoli, 2015). Self-administration and withdrawal from cocaine has been shown to increase the surface expression of CP-AMPA receptors (Ma et al., 2014; Wolf, 2010). Ingestion of palatable foods, including chocolate, has also been shown to induce long lasting increases in CP-AMPA receptors, especially in rats susceptible to obesity (Oginsky et al., 2016). These change in AMPA receptor surface expression have been associated with an increase in BDNF, a neurotropic factor involved in regulating of synaptic function and plasticity (Li \& Wolf, 2011).

\subsubsection{Immediate early genes}

Following long periods of withdrawal from an addictive substance, relapse can occur. One hypothesis is that this recurrence results from long-term changes in gene expression within the NAc. Notably, the activator protein-1 (AP-1) complex, composed of heterodimers of Fos and Jun family proteins, has received much attention (Karin et al., 1997). The Jun family consists of c-jun, junB, and junD and the Fos family consists of cfos, fra1, fra2, and FosB. The FosB gene encodes two proteins: FosB and DeltaFosB (Kelz and Nestler, 2000; Mumberg et al., 1991). All members of the Fos and Jun families are immediate early genes, meaning they are induced rapidly in response to numerous environmental stimuli, such as the ingestion of rewarding substances (McClung et al., 2004b). 
Cfos

The proto-oncogene c-fos is an immediate early gene that is a member of the Fos family, which can contribute to the formation of the AP-1 complex (Herrera and Robertson, 1996). C-Fos protein levels (52-55 kDa) within the NAc peak between 90120 minutes following the presentation of a stimulus (Chao and Nestler, 2004). Under basal conditions, neuronal levels of c-Fos are relatively low (Herrera and Robertson, 1996), hence c-Fos is often used as a measure of early neuronal activation because it induces rapidly but degrades within a few hours after induction (Hope et al., 1994).

The influx of calcium into a cell is necessary for c-Fos expression along with depolarizing stimuli that results in calmodulin proteins and protein kinase activity (Herrera and Robertson, 1996). It has been demonstrated that AMPA and NMDA receptor activation within the cortex and striatum, both of which have the potential to mediate calcium influx, can result in c-Fos induction (Herrera and Robertson, 1996). Administration of D1 agonists or D2 antagonists can also result in c-Fos induction within the striatum (Herrera and Robertson, 1996). The ability of glutamate and DA receptors to induce c-Fos suggests a role for cAMP in c-Fos induction.

\section{c-Fos and addictive substances}

Administration of cocaine and amphetamine has been shown to alter c-Fos expression within the NAcC and NAcSh, which is related to the context of administration and novelty of the substance (Larson et al., 2010; Renthal et al., 2008). Passive (experimenter administered) infusions of cocaine, both acute and chronic, has been shown to increase c-Fos protein levels within the NAcC and NAcSh (Larson et al., 2010). Cocaine that is administered in a response-contingent manner (self-administered) increases c-Fos in the NAcSh, but less so than passive administration, and does not increase c-Fos in the NAcC (Larson et al., 2010). Passive administration of amphetamine has also been shown to increase c-Fos protein expression (Renthal et al., 2008). However, repeated amphetamine administration results in decreased c-Fos activity, suggesting novelty induces a greater induction of c-Fos proteins (Renthal et al., 2008).

Chocolate delivery can affect c-Fos expression, particularly in response to chocolate anticipation (Blancas et al., 2014). A study by Blancas and colleagues (2014) investigated c-Fos induction in response to anticipation of daily chocolate administration (Blancas et al., 2014). The development of anticipation for chocolate was assessed over a period of 8 days (chocolate was given at the same time each day), and motivation to obtain chocolate was measured by approaches towards a box containing chocolate. An anticipatory activation of c-Fos was observed in the NAcC and NAcSh by day 5. This study shows that the anticipation of palatable food can result in c-Fos induction within rewarding brain regions when chocolate is given in a predictable pattern. This study also highlights that anticipation of chocolate, a highly palatable food to humans, has a strong ability to modify behaviour in rodents and activate reward related brain regions (NAc) (Blancas et al., 2014). 


\section{DeltaFosB}

DeltaFosB is a splice variant of FosB that lacks part of the c-terminal transactivation domain. Within the NAc, DeltaFosB is expressed predominantly in substance P/dynorphin-containing neurons, which mainly express D1 receptors (Kelz et al., 1999; Mumberg et al., 1991). During initial presentation with a rewarding stimulus, DeltaFosB has an unstable form at 33kDA which, over time, is altered to the more stable $35-37 \mathrm{kDa}$ form (Hope et al., 1994). The process by which this happens is unknown but is suggested to be the result of phosphorylation as DeltaFosB is phosphorylated on the serine residue (ser27) by casein kinase 2 (Hope et al., 1994; Nestler, 2012; Ulery, 2006). Long DeltaFosB isoforms are accumulated gradually following repeated stimulation and remain stable with half-lives that are estimated at weeks, whereas other members of the Fos and Jun families degrade quickly (Nestler, 2008). The stability of the long DeltaFosB isoform provides a molecular mechanism for drug-induced changes in gene expression to persist, even following long periods of withdrawal (Hope et al., 1994; Nestler, 2008; Nestler, 2012; Robison \& Nestler, 2011).

The longer-term DeltaFosB protein dissipates after 1-2 months while behavioural changes remain (Nestler, Barrot, \& Self, 2001). The thought is that DeltaFosB may induce long-term changes in receptor expression and neuronal structure (Nestler, 2005). The gene for the AMPA receptor subunit GluR2 contains an AP-1 site that binds the DeltaFosB protein (McClung et al., 2004b). When DeltaFosB is overexpressed in the NAc, the population of GluR2 AMPA receptors increases (McClung et al., 2004b). The GluR2 AMPA receptors show lower conductance compared to their GluR2-lacking, calcium permeable, counterparts, and may result in reduced glutamate neurotransmission within the NAc. Another target for DeltaFosB is the gene containing dynorphin whose release inhibits DA neurons that innervate MSNs (Wang et al., 2012). An accumulation of DeltaFosB is suggested to inhibit dynorphin, affecting the negative feedback loop and increasing activity at DA neurons (Wang et al., 2012). Lastly, DeltaFosB may affect neuronal structure via activation of $\mathrm{cdk} 5$, which regulates neuronal structure and growth (Wang et al., 2012).

DeltaFosB and Addictive substances

DeltaFosB has been shown to accumulate in the NAc substance P/dynorphin subset of MSNs following chronic administration of addictive drugs, most notably cocaine, and is associated with addictive-like behaviours (Kelz et al., 1999; Larson et al., 2010; Nestler, 2008; Nestler, 2004). This increase in DeltaFosB has been associated with an increase in the incentive motivation properties of cocaine, evidenced by mice working harder in a progressive ratio task to administer cocaine (Colby, Whisler, Steffen, Nestler, \& Self, 2003; Nestler et al., 2001). Increases in DeltaFosB expression have also been shown to increase the locomotor effects of cocaine, suggesting an enhanced sensitivity to cocaine contributing to addiction (Kelz et al., 1999).

Differences have been observed between the NAcC and NAcSh in the accumulation of DeltaFosB following acute and chronic cocaine administration (Larson 
et al., 2010). In the NAcSh, chronic (self-administration and passive administration), but not acute, administration of cocaine has been associated with an increase in DeltaFosB (Larson et al., 2010). In the NAcC, both acute and chronic administration (passive) of cocaine has been associated with an increase in DeltaFosB (Larson et al., 2010).

Similar to drugs of abuse, natural rewards have been shown to cause similar increases in DeltaFosB (Hadad and Knackstedt, 2014; Pitchers et al., 2013; Sharma et al., 2013; Teegarden et al., 2009; Velázquez-Sánchez et al., 2014a). Mice fed a high fat diet show increased DeltaFosB within the NAc compared to mice fed a low fat diet (Sharma et al., 2013). These animals also exhibited enhanced motivation for sucrose and high fat food rewards following withdrawal (Sharma et al., 2013). Further, rats that are selected as highly impulsive, and trained to administer palatable food, show an increase in expression of DeltaFosB within the NAcSh that is higher than low-impulsive rats (Velázquez-Sánchez et al., 2014a). This suggests a potential predisposition to the development of DeltaFosB over expression in response to palatable foods.

\subsubsection{Synaptic plasticity}

Long-term synaptic changes in the brain may be the basis of learning and memory at the cellular level (Hebb, 1949). Experience-dependent plasticity, which includes learning and memory, are examples of activity-dependent changes at excitatory synapses that result in a change in synaptic strength (Malinow and Malenka, 2002). These changes in synaptic strength result from altering the excitability of neurons by changing the strength of connections that already exist, which may lead to the formation and/or elimination of synapses and remodelling of dendrites and axon (Hebb, 1949).

Synaptic plasticity can be subdivided into short-term and long-term plasticity (Citri and Malenka, 2008; Zucker and Regehr, 2002). Short-term plasticity can last anywhere from milliseconds to minutes and plays a role in sensory adaptations, behavioural changes, and short-term memory (Zucker and Regehr, 2002). Short-term plasticity can result from short bursts of activity within presynaptic neurons affecting the probability of neurotransmitter release (Citri and Malenka, 2008). Long-term synaptic plasticity results from activity-dependent modifications of synaptic strength (Citri and Malenka, 2008). These long-term activity-dependent changes may result from activation of NMDA receptors and alterations in AMPA receptor density and sub-unit composition (Citri and Malenka, 2008).

Under normal circumstances, synaptic transmission at glutamate synapses is mediated via $\mathrm{Na}+$ influx through AMPA receptors (Kessels and Malinow, 2009; Malinow and Malenka, 2002). Long-term depression (LTD) and long-term potentiation (LTP) are examples of synaptic plasticity and have been extensively studied within the hippocampus, but less so within the NAc (Malinow and Malenka, 2002). LTP is the long-term strengthening of a synapse from prolonged stimulation and LTD is a long-term weakening of a synapse from less intense stimulation (Colbran, 2015). LTP can be triggered by a rise in postsynaptic $\mathrm{Ca} 2+$ as a result of NMDA receptor activation (Malinow and Malenka, 2002). Depolarization of the post synaptic membrane is necessary for NMDA receptors to expel a $\mathrm{Mg} 2+$ ion, which is a requirement for $\mathrm{Ca} 2+$ 
and $\mathrm{Na}+$ influx to occur, along with activation of the receptor with glycine and glutamate (Citri and Malenka, 2008). The influx of $\mathrm{Na}+$ and $\mathrm{Ca} 2+$ can increase postsynaptic depolarization, increasing synaptic strength and the potential for LTP (Citri and Malenka, 2008).

There is strong evidence that LTP associated with craving involves the insertion of new CP-AMPA receptors into the post synaptic membrane (Malinow and Malenka, 2002). For example, addiction related behaviours have been shown to be mediated via AMPA receptor trafficking in the mesolimbic DA system (Kessels and Malinow, 2009). Withdrawal from cocaine correlates with an increase in GluR1 protein levels within the NAc (Conrad et al., 2008). This would increase Ca2+ within the NAc and may sensitize the NAc neurons to cocaine associated cues, thus promoting craving (Conrad et al., 2008). Antagonism of GLuR1 containing AMPA receptors decreases drug seeking behaviour providing further support for the role of CP-AMPA receptors in craving (Zheng et al., 2015).

\subsubsection{Structural Plasticity}

Structural plasticity consists of changes in the number of synapses, synaptic connections, and dendritic branching, which arise from the formation of new synapses or the removal of old ones (Butz et al., 2009). The activity-dependent rewiring of synapses can result in an increase in spine density, which can result in a functional synapse similar to mature spines (Fauth and Tetzlaff, 2016). Dendritic spines are points of synaptic contact that were first described by Ramon y Cajal from observations of Purkinje cells, which were visualized with the Golgi staining method (Cajal, 1888, 1891). Spine morphology is heterogeneous in nature, but mature spines frequently consist of a bulbous head and a thinner neck that connects the spine to the dendrite (Svoboda et al., 2017; Wilson et al., 1983). The head of a spine consists of postsynaptic density, defined as receptors and other supporting proteins, which can change in structure and composition in response to synaptic activity (Spiga et al., 2014).

When a dendritic spine changes in shape, it may be associated with either shortor long-term plasticity (Kasai et al., 2010). The formation of new dendritic spines and enlargement of existing ones is associated with LTP, whereas LTD is associated with either a shrinkage of existing spines or complete retraction (Nägerl et al., 2004; Okamoto et al., 2004). LTP and LTD result in changes to signalling pathways related to the synthesis of cytoskeletal proteins, altering the polymerization of actin, which affects spine maturation and stability (Tada and Sheng, 2006). Also, blockade of NMDA receptors prevents influx of $\mathrm{Ca} 2+$ into the cell and has subsequently been shown to prevent dendritic spine changes (Yasumatsu et al., 2008).

Dendritic spines are divided into classes depending on their morphology, these classes include mushroom, stubby, thin, and filipodia type spines (Ghani et al., 2016). Mushroom spines have large bulbous heads and long necks, stubby spines are missing a neck, thin spines have small heads and thin necks, and filipodia have long necks without clear heads (Ghani et al., 2016). The thin and mushroom type spines are suggested to be the most important in excitatory synaptic activity (Spiga et al., 2014). Mushroom type 
spines appear to be more stable and can persist for months and thin spines tend to change more rapidly (Bourne and Harris, 2007; Huber and Menzel, 2004). For example: cocaine treatment results in small spines becoming larger (e.g. synapse strengthening) and withdrawal results in spines becoming smaller with an increased density (Robinson, 1999; Spiga et al., 2014)

A popular method for the quantification of dendrites and dendritic spines is the Golgi-cox staining procedure. The Golgi method stains $1-4 \%$ of neurons at random providing reliable and extensive staining (Kolb and Whishaw, 1998). Staining using the Golgi procedure allows the measurement of dendritic length and the estimation of dendritic spine density. Dendritic changes represent an indication of change because they can expand and retract in response to various stimuli (Kolb and Whishaw, 1998). Measuring the characteristics of dendritic spines, such as density and shape, is informative since the majority of excitatory synapses form on spines (Kolb and Whishaw, 1998).

Dendritic Spines and Craving

Alterations in dendritic spines, such as density and morphology, has been implicated in food and drug-seeking behaviour (Guegan et al., 2013; Kobrin et al., 2016; Nyberg, 2014; Quintero, 2013). Chronic self-administration or experimenter administration of many drugs, including amphetamine and cocaine, is associated with increased spine density on MSN's in the NAcSh and NAcC (Robinson \& Kolb, 2004; Robinson, 1999). This increase in spine density can be seen months after amphetamine has been discontinued and has also been shown to persist a month after cocaine administration (Robinson \& Kolb, 2004). Self-administration of highly palatable food has also been shown to increase dendritic spine density within the NAcSh (Mancino et al., 2016). These long-term plastic changes are correlated with behaviours associated with drug use, such as craving, which may lead to relapse (Nestler, Hope, \& Widnell, 1993; Robinson \& Berridge, 1993; Robinson \& Kolb, 2004).

\subsection{Craving as a learned response}

The salience (attentional bias) of environmental cues has been shown to increase when food is restricted, such as when dieting, increasing the possibility of craving (Durkin et al., 2012; Hollitt et al., 2010; Placanica et al., 2002). Learning-based theories of addiction have attempted to explain the change in salience that may occur to addiction related cues (Robinson \& Berridge, 2000; Robinson \& Berridge, 2008). Prominent learning theories of addiction are based on classical and operant learning principles and attempt to explain how addiction comes about as a learned habit (Wanigaratne, 2006).

\subsubsection{Classical Conditioning Principles}

Pavlovian or classical conditioning involves pairing two stimuli: the unconditioned stimulus (US) and the conditioned stimulus (CS) (Domjan, 2005). The US results in a physiological response in the absence of training, and the CS results in little 
or no initial response (Domjan, 2005). The ability of the CS to produce a response depends on the predictive value of the US (Domjan, 2005). When learning has occurred, the CS will be able to elicit a similar response to the one elicited by the US; a conditioned response (CR) (Domjan, 2005).

The ability of an unrelated US and CS to become associated is the most basic form of associative learning mechanisms (Domjan, 2005). Food was the first US used in conditioning studies, as it was recognized that anticipation of meat powder could result in salivation, and that ringing a bell in concert with meat powder could result in an association, where eventually ringing the bell alone resulted in salivation (Domjan, 2005; Pavlov, 2010). The anticipation of food, as represented by the CS that was previously paired with food, can begin to trigger digestive responses, when food is anticipated, even in cases where it is not present (Domjan, 2005). Besides excitatory stimuli, classical conditioning principles can also be applied to aversive learning. In taste-aversion, the paired CS and US result in delivery of lithium chloride, which makes rodents sick. The rodents then learn to avoid the lithium chloride when presented with the CS (Garcia et al., 1955).

\section{Classical Conditioning and Craving}

Classical conditioning principles have been used to explain the acquisition of drug/food seeking behaviours and craving (Wanigaratne, 2006). In terms of drug use, previously neutral stimuli are associated with the drug response, which can subsequently lead to increased craving and relapse when an addict is re-exposed to the environment that has been associated with the drug response (Wanigaratne, 2006). Food seeking behaviour has also been shown to increase when an individual is in an environment that was previously associated with a palatable food (Morris et al., 2015). Everyday occurrences such as food advertisements on television and billboards may trigger craving for palatable food, particularly in susceptible individuals.

Classical conditioning studies related to substance use involve passive substance delivery, where the experimenter delivers a substance and the rodent does not have to perform any task for substance delivery. Chronic substance delivery, in a non-contingent manner, can result in behavioural sensitization to environmental cues, affecting subsequent drug self-administration in operant tasks (Van den Oever, 2012). Thus, behavioural sensitization may facilitate relapse after periods of abstinence in selfadministration models and result in sensitivity to drug-associated cues (Van den Oever, 2012). Drug use in humans is usually not subject to the control of the experimenter, but classical conditioning can be applied to both drug seeking and drug taking. Physiological changes that result in feelings of euphoria (i.e. increased heart rate), may occur repeatedly in the same environment where specific environmental cues are present, such as, people, sounds, and odours (Obrien et al., 1992). These environmental cues may later act as conditioned stimuli, resulting in drug seeking and drug taking behaviour (Obrien et al., 1992).

In terms of food, there is evidence that overweight and non-overweight individuals differentially acquire strong classically-conditioned associations to previously 
neutral cues that have been paired with palatable food (Burger and Stice, 2011; Meyer et al., 2014). For example: A study by Meyers and colleagues (2015) compared classical conditioning to food cues in overweight and normal weight individuals (Meyer et al., 2014). Participants were seated in a reclining chair with a computer screen in front of them and gustometer for food delivery. The acquisition of a swallowing response, measured with electromyography (EMG), to neutral visual cues presented on the computer screen (circle, square, and rectangle) was explored. Neutral visual cues were paired with chocolate milk, water, or no taste (Meyer et al., 2014). There were 27 acquisition trials, each was followed by a 20 second inter-trial interval. The acquisition phase was followed by 27 extinction trials where the presentation of a cue did not result in a reward (Meyer et al., 2014). The results revealed that overweight individuals had a heightened swallowing response to cues paired with chocolate milk compared to cues paired with water, whereas normal weight participants did not show this heightened cue discrimination (Meyer et al., 2014). The overweight individuals also showed difficulty extinguishing the learned response (swallowing) to the cues (Meyer et al., 2014).

\subsubsection{Operant Conditioning Principles}

In 1937, B. F. Skinner described behaviour that is "controlled by its consequences" and termed it "operant behaviour", and the learning behind the behaviour “operant conditioning” (Skinner, 1937; Staddon \& Cerutti, 2003). Operant conditioning is goal-directed behaviour that results in a specific environmental outcome. Operant conditioning may involve several different schedules of reinforcement, which can be divided into two types: ratio and interval. The amount of time between presentation of the reinforcer can also be on a variable or fixed interval schedule and the amount of responses necessary to obtain a reward can be on a variable or fixed ratio schedule (Staddon and Cerutti, 2003). The strength of the association between the operant and behaviour can be tested using extinction, where the reward is no longer presented in response to a specific behaviour. If an association is strong it will take longer to extinguish the behaviour.

\section{Operant Conditioning and Craving}

Operant conditioning principles may also be used to explain aspects of craving. The premise of operant conditioning is that the use of reinforcement will increase the chance that a behaviour will be repeated (Staddon and Cerutti, 2003). Positive reinforcement occurs when a behaviour is emitted that results in gaining a reward, whereas negative reinforcement occurs when a behaviour is emitted that results in the removal of a negative stimulus; both positive and negative reinforcement result in increased behaviour (Staddon and Cerutti, 2003). In operant conditioning, reinforcement refers to a contingency that is involved in strengthening a behaviour (Salamone, Correa, Farrar, \& Mingote, 2007). In terms of addiction, a drug can serve as both a positive and negative reinforcer depending on the situation (Wanigaratne, 2006). Initially, drugs are positively reinforcing (during initial binge/intoxication phase), where an individual takes a drug for the feeling of euphoria (Wanigaratne, 2006). During the withdrawal phase of 
addiction, the removal of the aversive symptoms via drug taking is negatively reinforcing (Wanigaratne, 2006).

Operant conditioning tasks have been used to show the effects of food reinforcement in humans (Nasser et al., 2008; Watson et al., 2014). A study by Nasser and colleagues (2008) investigated food reinforcement in human with and without binge eating disorder using an operant task (Nasser et al., 2008). The task consisted of a computer generating a progressive ratio schedule, where participants were credited $1 \$$ amount of food or non-food items that were available after the task. Some participants consumed a liquid meal before the task or they received flavoured water to determine the effect on operant responding. Control participants that received the liquid meal prior to operant conditioning showed decreased operant responding than those who received water. However, the binge eating group responded more during the operant task after consuming the liquid meal compared to the control group (Nasser et al., 2008). This study provides evidence that susceptible individuals may continue to eat even when they are not physically hungry in response to food reinforcement (Nasser et al., 2008). Similarly, when individuals are satiated prior to presentation of cues that were related to chocolate and popcorn consumption, there is still an increased responding for the food reward (Watson et al., 2014).

Animal models of drug addiction often involve a combination of classical and operant conditioning principles. This is seen in self-administration models where pressing a bar for a drug results in the administration of the drug paired with a discrete cue, such as a tone or light, this is the conditioned stimulus. Operant self-administration models of addiction most resemble human drug addiction, as animals have control over substance intake (Van den Oever, 2012). An operant response (such as a lever press or nose poke) will result in activation of a syringe pump or presentation of a food pellet (Van den Oever, 2012). The delivery of a substance can be associated with environmental cues (such as a tone and/or light), that will become associated with the rewarding effects of a substance (Van den Oever, 2012). Operant models can also be used to test relapse following extended periods of abstinence, where substance seeking is triggered by exposure to environmental cues that were previously paired with a rewarding substance, which is also seen in human addicts (Epstein et al., 2006; Van den Oever, 2012).

Operant learning theories explain a part of the addiction process, but may not explain the craving that contributes to compulsive drug actions after long periods of abstinence (Robinson \& Berridge, 2008). Prominent learning theories focus on stimulusresponse habits (Hyman et al., 2006), but it is difficult to consider how this alone could lead to compulsive behaviour (Robinson \& Berridge, 2008). It is suggested that strong stimulus-response behaviours may not necessarily lead to compulsive behaviour, and additional motivational mechanisms may be necessary to explain addiction (Robinson \& Berridge, 2008). A stimulus-response habit would imply that an addict would search for their drug of choice in the same manner every time; however, this is not the case as addicts can be quite resourceful (Robinson \& Berridge, 2008). Hence, the main question of drug craving is why can it persist long after physiological withdrawal has subsided (Robinson \& Berridge, 1993). 


\subsubsection{DA Hypothesis of reinforcement}

Learning can become maladaptive in drug addicted individuals possibly via alterations within the mesolimbic DA system (Day \& Carelli, 2007; Wise \& Rompre, 1989; Wise, 1988; Wise, 1996; Wise, 2006). The main premise of the DA hypothesis of reinforcement is that the DA system mediates the reinforcing effects of pleasurable stimuli (Salamone \& Correa, 2002). An important component of the DA hypothesis is that the reinforcing effects of brain stimulation and drugs of abuse activate natural reward systems (Salamone \& Correa, 2002). These reward systems include DA synapses and DA receptor containing neurons in the NAc which are involved in the reinforcing effects of natural stimuli, such as food (Salamone \& Correa, 2002). Eventually, presentation of the conditioned stimulus, without the unconditioned stimulus, can trigger DA release within the NAc (Day and Carelli, 2007). The ability of a conditioned stimulus to increase DA is an important factor in craving, and subsequent relapse, in addiction.

The study of operant and classical conditioning principles at the neural level has been accomplished by observing synaptic connections and strengthened neural responses (Wise, 2004). When an organism is presented with a unconditioned, conditioned, or reinforcing stimulus, DA is released within limbic structures such as the NAc (Guerra and Silva, 2010). In operant conditioning, NAc neuron firing rate changes when animals respond for reward (Carelli and Deadwyler, 1994). Learning will not occur if DA antagonists are administere prior to learning, but if DA antagonists are given after learning, learning will still occur (Wise, 2004).

Pharmacological studies have been used to identify the importance of NAc DA in the reinforcing effects of natural reinforcers (Geary \& Smith, 1985; Wise, Fotuhi, \& Colle, 1989; Wise, Spindler, \& Legault, 1978). DA receptor antagonists have been shown to reduce the reinforcing effects of food in hungry animals (Geary \& Smith, 1985; Wise et al., 1978). Direct injection of amphetamine into the NAc has been shown to facilitate feeding (Wise et al., 1989).

DA activity in the NAcC and NAcSh have been shown to differ in response to conditioned stimuli (Day and Carelli, 2007). Microdyalisis studies have determined that food reward increases DA concentration within the NAcSh (Bassareo and Di Chiara, 1997, 1999). Conditioned stimuli that was previously paired with food or cocaine reward results in an increase in DA concentration in the NAcC, but not the NAcSh (Bassareo and Di Chiara, 1997, 1999; Ito et al., 2000). Lesions to the NAcC have been shown to disrupt associations between unconditioned and conditioned stimuli, a similar effect was seen with DA antagonism of the NAcC (Day and Carelli, 2007). This suggests that NAcC DA transmission may mediate associative learning while NAcSh DA transmission may be activated in response to primary reinforcement (Di Chiara, 2002).

The novelty of a stimulus can differentially affect DA release within the NAcC and NAcSh. DA release within the NAcSh corresponds with ingestion of novel palatable food, which habituates over time (Di Chiara, 2002). Unlike palatable food, stimulation of NAcSh DA by drugs does not undergo habituation, possibly due to the motivational properties of the drug (Di Chiara, 2002). Food restriction can prevent habituation of the DA response within the NAcSh (Di Chiara, 2002), individuals restricting food intake in 
an attempt to lose weight may have a similar increase in incentive value of food as is seen with drugs of abuse, resulting in continued maladaptive eating behaviours.

Glutamate neurotransmission within the NAc has been suggested to modulate activity at MSN's (Wise, 2004). There is evidence for a role of inotropic glutamate receptors in responding for conditioned stimuli (Quirk and Mueller, 2008). MK-801 is a non-competitive NMDA receptor antagonist that binds to the PCP site within the ion channel, thus preventing the influx of Ca2+ into the cell (Wong et al., 1988). In order for MK-801 to bind, the post-synaptic membrane must be depolarized and the $\mathrm{Mg} \mathrm{2+}$ ion expelled (Nicoll and Malenka, 1999; Nowak et al., 1984). When MK-801 is given acutely, prior to an operant extinction task, where bar pressing results in cue presentation but no food, rats bar press at a higher rate than controls (Davis-MacNevin et al., 2013; Holahan et al., 2010, 2012; Tuplin et al., 2015). This behavioural perseveration represents maladaptive food seeking behaviour, not unlike what is seen in drug addiction models.

\subsection{Behavioural Measures of Craving}

In order to study drug addiction and its neural correlates, animal models are used. Rodent models are useful for understanding the function and structure of brain regions because they provide simpler systems, at the same time as maintaining complex behavioural characteristics that are important to the understanding of addiction processes (Kesner \& Churchwell, 2011). Animal models of addiction can be used to measure behavioural changes that are reminiscent of addiction in humans (Van den Oever, 2012). An appropriate animal model will have face validity, predictive validity, construct validity, and external validity, in order to translate to the human condition (Van den Oever, 2012). Rodent models have been shown to have qualities of face validity, due to the homologous neural structures within the mesocorticolimbic system between humans and rodents (Childress et al., 1999; Goldstein \& Volkow, 2011; Hyman, 2005a; Peters et al., 2009b). Predictive validity is represented in rodent models (Epstein et al., 2006), but may not necessarily lead to effective treatment strategies in patients (Van den Oever, 2012). Rodent models that involve food and drug self-administration attempt to mimic the pathology, symptomatology, and aetiology of addiction, in order to represent appropriate construct validity (Van den Oever, 2012). Rodent self-administration models are also good at representing external validity, as aspects can be translated to the human condition (Epstein et al., 2006).

Animal models of addiction tend to follow two patterns of substance administration: contingent (self-administration; most similar to human addiction) or noncontingent (passive exposure; experimenter administered) (Van den Oever, 2012). Operant conditioning principles can be employed to represent contingent administration where animals press a lever to self-administer a substance. Classical conditioning principles can be employed to represent a more passive or non-contingent drug administration, as animals do not change their behaviour to self-administer a substance. Rather, it is presented into the environment by the experimenter. 


\subsubsection{Classical conditioning}

Classical conditioning, as described earlier, represents a non-contingent method of food delivery. Chocolate delivery is not contingent upon the behaviour of the rat; rather, chocolate is delivered at random time-intervals.

\section{Classical Conditioning procedure}

Classical conditioning will involve rats being trained in operant chambers equipped with a house light, cue lights, tones, food dispenser and a metal-gridded floor. During acquisition sessions, rats will be placed in the operant chambers (Coulbourn Instruments; $30.5 \mathrm{~cm} \mathrm{~W} \mathrm{x} 25.5 \mathrm{~cm} \mathrm{D} \mathrm{x} 30.5 \mathrm{~cm} \mathrm{H}$ ) for 1 hour where one $45 \mathrm{mg}$ chocolate pellet will be dispensed on a variable interval 30 -second schedule. The house light extinguishing and the panel lights changing from red to green ( 5 seconds) will be associated with the presentation of the chocolate pellet. The classical conditioning trials will occur over ten days (one trial per day). Nose pokes and locomotor activity will be recorded and rats will be grouped based on nose pokes during day 10 of conditioning.

Following acquisition rats will be tested in a cue-induced responding paradigm. Rats will be tested in the same operant chambers where they underwent classical conditioning the only difference being that a correct and incorrect lever will be introduced into the chambers. The correct lever will result in presentation of the cue (house light extinguishing and panel lights changing from red to green for 5 seconds) that was previously associated with the presentation of a chocolate pellet. Correct presses, incorrect presses, nose pokes, and locomotor activity will be recorded at 10-minute intervals.

\subsubsection{Operant Conditioning}

Operant conditioning, as described earlier, represents a contingent method of food delivery. The delivery of chocolate is contingent upon the rat pressing a lever.

\section{Operant Conditioning Procedure}

Operant studies within our laboratory involve rats being trained in operant chambers equipped with a house light, cue lights, tones, food dispenser, metal-gridded floor, and two levers (one that dispenses food and one that does not). During acquisition sessions, rats will be placed in the operant chambers (Coulbourn Instruments; $30.5 \mathrm{~cm} \mathrm{~W}$ x $25.5 \mathrm{~cm} \mathrm{D} \mathrm{x} 30.5 \mathrm{~cm} \mathrm{H}$ ) for 1 hour where every second response (FR2 schedule) on the correct lever results in the delivery of a $45 \mathrm{mg}$ chocolate pellet (BioServe, New Jersey). Once the left lever (correct lever) had been depressed twice, the house light extinguishes, the panel lights above the lever change from red to green (for 5 seconds), and the pellet dispenser releases one chocolate pellet. The right lever (incorrect lever) has no programmed consequences. The acquisition trials will occur over ten days (one trial per day). At the end of each acquisition trial the total correct presses, incorrect presses, nose 
pokes, and locomotor activity will be recorded. Rats will be grouped based on total correct presses during day 10 of acquisition.

Once the behaviour has been established, the behaviour can be extinguished. The extinction session will be 60 minutes and bar pressing will no longer result in food reward; however, the house light will extinguish and the panel lights will change from red to green for 5 seconds. Bar pressing will no longer result in food reward, but the presentation of the discrete cue (house light extinguishing and panel lights changing from red to green) should remain rewarding due to its previous association with food reward. Correct presses, incorrect presses, nose pokes, and locomotor activity will be recorded at 10-minute intervals.

\subsubsection{Conditioned Place Preference}

Conditioned place preference is a behavioural procedure that employs classical conditioning principles. Chocolate is paired with a specific environment, such as a black or white compartment. The environment represents a conditioned stimulus that was previously paired with chocolate. If the rat develops an association between the environment and chocolate, given a choice between compartments, the rat will spend more time in the compartment previously paired with chocolate.

\section{Conditioned Place Preference Procedure}

The CPP apparatus consists of two chambers separated by a small walkway. One chamber is white with a smooth floor and the other chamber is black with a gridded floor, the middle chamber is gray. Removable grey doors separate the middle walkway from the two chambers.

An unbiased experimental design will be used, and rats will be counterbalanced so that some animals receive milk chocolate or chocolate flavoured pellets on the white smooth side and some animals receive the food on the black gridded side. A control group will be run through the entire procedure but will not received food on either side. The control group is used to determine that there is no natural bias for either compartment. A habituation day will be conducted prior to conditioning where rats will be allowed to roam the entire chamber for 20 minutes to acclimate them to the environment. Following habituation rats will undergo 10 days of conditioning for 20 minutes a day: on days $1,3,5,7$, and 9 rats will be placed on the unpaired side and will not receive any food, and on days $2,4,6,8$, and 10 rats will be placed on the paired side and given $5 \mathrm{~g}$ of either chocolate flavoured pellets or milk chocolate chips. During testing rats will be placed in the middle compartment and have access to the entire apparatus for 20 minutes. Testing will be video-recorded and the amount of time spent in each compartment will be calculated. A difference score will be calculated by subtracting the unpaired side from the paired side. A score above 0 represented a preference and a score below 0 represented an aversion. 


\subsection{Incubation of craving}

Relapse following a period of withdrawal from an addictive substance is often associated with craving, a poorly understood phenomenon that precedes drug-seeking behaviours (Childress et al., 1999). In human drug addicts, environmental cues can precede relapse. These environmental cues include the drug itself, sights, sounds, odours, and other situations that are associated with drug use (Obrien et al., 1992). Exposure to these environmental cues may evoke physiological changes that embody craving and contribute to subsequent drug seeking behaviour (Obrien et al., 1992).

Craving, or preoccupation with a substance, may continue even after physiological withdrawal symptoms have subsided (Lu et al., 2004; Pickens et al., 2011). Long-term changes in motivational circuitry following self-administration of palatable substances may contribute to relapse (Grimm et al., 2005). Animal research has provided evidence that suggests the onset of craving may be delayed, and even enhanced, following a period of abstinence from a substance (Grimm et al., 2001). In animal models, craving is defined as an increase in responding for a substance-related cue, in the absence of that substance. Following a period of abstinence from cocaine, rats have shown increased cue-induced responding on day 30 compared to day 1 of withdrawal (Aoyama et al., 2014; Li and Frantz, 2009; Lu et al., 2005). This enhanced and delayed craving following a period of abstinence that is increased in a time-dependent manner is termed "incubation of craving" (Guillem et al., 2014; Lu et al., 2004; Pickens et al., 2011).

Currently, the majority of studies investigating the incubation of craving have explored cocaine (Chauvet, Goldberg, Jaber, \& Solinas, 2012; Conrad et al., 2008; Grimm, Shaham, \& Hope, 2002; Halbout, Bernardi, Hansson, \& Spanagel, 2014; Hale et al., 2001; Lu et al., 2005; Ma et al., 2014; Wolf \& Tseng, 2012) with a few studies delving into other addictive substances such as methamphetamine (Krasnova et al., 2014; Li, Zeric, Kambhampati, Bossert, \& Shaham, 2014), the natural reinforcer sucrose (Grimm, Manaois, Osincup, Wells, \& Buse, 2007; CLi \& Frantz, 2009, 2009; Li, Caprioli, \& Marchant, 2014; Pickens et al., 2011), high fat and regular food pellets (Darling et al., 2016; Dingess et al., 2017a). The incubation of craving phenomenon has been attributed to long-term changes in rewarding brain pathways, which include the NAc (Counotte, Schiefer, Shaham, \& O’Donnell, 2014; Ferrario et al., 2011; Li et al., 2013; Wolf \& Tseng, 2012) and PFC (Fanous et al., 2012; Koya et al., 2009a; Theberge et al., 2012). It is hypothesized that these regions change in a time-dependent manner both structurally and functionally after a period of withdrawal from a rewarding substance (Pickens et al., 2011). These changes include alterations in receptor composition (AMPAr) (Counotte et al., 2014; Ferrario et al., 2011; Wolf and Tseng, 2012) and the expression of growth factors (BDNF) (Li et al., 2013).

\subsubsection{Models}

\section{Self-administration models}


Current preclinical models of addiction have focussed on the later stages of the addiction cycle, which include compulsive cue-controlled drug seeking habits that result in compulsive relapse (Belin-Rauscent et al., 2015). There are several variations of cueinduced classical conditioning paradigms used to study the craving phenomenon. Most prominent is a rodent model of relapse where rats are trained to self-administer a rewarding substance for several days. An operant chamber is used where lever pressing results in the reward and the presentation of a tone and light stimulus. During testing, craving is defined as responding on the correct lever in a testing session where depressing the lever results in the tone + light stimulus, but no presentation of the reward. The tone and light pairing represents reinforcing properties of the previously presented rewarding substance. Pressing an incorrect lever represents the ability to discriminate between levers, and also as a measure of non-specific motor activity along with photo beam breaks. This general testing method for the incubation of craving can be varied to include: satiation prior to testing, environmental enrichment during the abstinence period, and pharmacological interventions (Grimm, Barnes, North, Collins, \& Weber, 2011).

\section{Cocaine}

The most prominently studied drug in the incubation of craving is cocaine.

Reports have consistently shown a time-dependent increase in cocaine seeking following a period of abstinence in cue-induced reinstatement models (Grimm et al., 2002; Hale et al., 2001; Li \& Frantz, 2009; Lu et al., 2005). Grimm and colleagues (2002) investigated the incubation of craving phenomenon using extinction testing in both cocaine and sucrose treated rats, after 1 day and 15 days of abstinence (Grimm et al., 2002). Rats were trained to self-administer cocaine $(0.5 \mathrm{mg} / \mathrm{kg} /$ infusion $)$ for two, 3-hour sessions a day for 10 days with a 40 second time-out after each reward (Grimm et al., 2002). A 5second tone-light cue was presented with each presentation of reward and rewards were limited to 40 per $3 \mathrm{hr}$. session (Grimm et al., 2002). The cue-induced reinstatement test consisted of a 1-hour session where responses resulted in presentation of the tone-light cue with a 40 second time-out. Rats tested on day 15 of withdrawal responded significantly more for the tone-light cue than rats tested on day 1 in both the cocaine and sucrose groups; although the incubation effect for cocaine was more pronounced (Grimm et al., 2002).

Hale and colleagues (2001) used a similar procedure and tested for cue-induced cocaine responding after $1,2,4,7,15,29$, and 60 days of abstinence from cocaine (Grimm et al., 2001). Cue-induced responding continued to increase throughout the withdrawal period, suggesting that an individual may be more vulnerable to relapse two months after cessation of cocaine (Grimm et al., 2001). A stud by Li and colleagues (2009) found a similar result where cue-induced responding continued to increase over the abstinence period of 60 days (Hale et al., 2001; Li \& Frantz, 2009). Compared to Hale and colleagues (2001), Li and colleagues (2009) used a lower dose of cocaine (0.36 $\mathrm{mg} / \mathrm{kg} /$ infusion), and less training time, where animals were trained for 2 hours a day for 14 days instead of 6 hours a day for 10 days (Li \& Frantz, 2009). Despite the differences in training and drug dose, the incubation effect was similar in these studies (Grimm et al., 2002; Hale et al., 2001; Li \& Frantz, 2009). 


\section{Other drugs and palatable substances}

The experimental design used to study the incubation of cocaine craving has been expanded to include methamphetamine (Li et al., 2015; Li, Zeric, et al., 2014)), sucrose (Grimm et al., 2005), high fat (Darling et al., 2016; Dingess et al., 2017a), regular show (Darling et al., 2016; Dingess et al., 2017a), and a 66\% carbohydrate palatable pellet (Krasnova et al., 2014). Li and colleagues (2014) investigated the incubation of methamphetamine craving by training rats to self-administer for $9 \mathrm{~h} / \mathrm{day}(0.1 \mathrm{mg} / \mathrm{kg}$ infusion) over 10 days and running cue-induced reinstatement tests following 2 days or 1 month of withdrawal. Following 1 month of withdrawal, cue-induced bar pressing, where no methamphetamine was delivered, was significantly greater than after 2 days of withdrawal (Li, Zeric, et al., 2014). Grimm and colleagues (2005) studied the incubation of sucrose craving where rats were trained for $6 \mathrm{~h} /$ day for 10 days to self-administer liquid sucrose (Grimm et al., 2005). Rats were tested for cue-induced responding after 1, 7, and 30 days of withdrawal from sucrose. Similar to rats that were trained to self-administer cocaine and methamphetamine, there was a time-dependent increase in cue-induced responding, with greater responding at 30 days of withdrawal compared to 1 day (Grimm et al., 2005). When the training time for sucrose self-administration was reduced from 6 to 2 hours, a time-dependent increase in cue-induced responding remained following 30 days of withdrawal (Grimm et al., 2005). The incubation of craving for palatable food (high fat pellet), and methamphetamine, was assessed in a study by Krasnova and colleagues (2014). Rats were trained to self-administer palatable food for $9 \mathrm{hrs}$./day for 14 days, where bar pressing resulted in the delivery of five 45-mg "preferred" food pellets along with a tone-light cue (Krasnova et al., 2014b). During cue-induced reinstatement testing, rats pressed more on day 21 compared to day 2 of withdrawal from palatable food and methamphetamine (Krasnova et al., 2014b). This suggests that both food and drug withdrawal can result in substance seeking behaviour following 21 days of forced abstinence, suggesting similar underlying neural mechanisms.

\section{Non-contingent (passive delivery) models}

The self-administration studies indicate that the incubation of craving may be generalizable to many addictive and palatable substances, especially when substance presentation is contingent on self-administration. There are limited studies that explore the incubation of craving with respect to passive substance delivery (Diehl et al., 2013b; Li et al., 2008; Lubbers et al., 2015; Sun et al., 2017). Diehl and colleagues (2013) used a model of cue-induced conditioned activity to determine if the locomotor responses to a non-contingent drug presentation sensitizes (i.e., show incubation of craving response) during withdrawal (Diehl et al., 2013a). A conditioned association to $15 \mathrm{mg} / \mathrm{kg}$ of cocaine was made with a discrete tone-light cue over 12 conditioning sessions. Cueinduced conditioned activity was assessed following 3, 14, and 28 days of withdrawal from cocaine. Rats had consistent locomotor activity across all 3 withdrawal days and did not show an incubation effect (Diehl et al., 2013a). Other non-contingent models of the incubation of craving utilize CPP to determine if rewarding contextual cues can induce an incubation of craving response. Using unbiased CPP designs, CPP scores have been shown to time-dependently increase following forced abstinence from morphine and 
cocaine (Li et al., 2008; Lubbers et al., 2015), while another study showed a time dependent decrease following morphine abstinence (Sun et al., 2017). These studies that utilize non-contingent models of the incubation of craving suggest that non-contingent models are less consistent in resulting in an incubation of craving response. However, more studies on non-contingent vs contingent substance administration are necessary to make this conclusion.

\subsubsection{Experimental Manipulations (environmental enrichment)}

Environmental enrichment has been shown to reduce cocaine seeking in selfadministration models of addiction, similar to those previously described (cocaine selfadministration 6/hrs./day for 10 days or 15 days) (Chauvet et al., 2009; Thiel et al., 2010). Environmental enrichment in cocaine models of addiction involves a larger home cage, group housing, toys, and a running wheel. Following 30 days of environmental enrichment and forced abstinence from cocaine, cue-induced cocaine seeking was reduced compared to animals housed in a standard environment (Chauvet et al., 2009). The assessment of environmental enrichment in the incubation of cocaine craving has revealed an increase in cue-induced responding in both standard environment and environmental enrichment groups, but the environmental enrichment groups showed significantly less cue-induced responding at day 30 of withdrawal compared to standard environment groups (Chauvet et al., 2012).

The effect of lithium chloride on sucrose aversion in the incubation of sucrose craving was assessed in a study by Harkness and colleagues (Harkness et al., 2010). Rats were trained to self-administer sucrose paired with a tone-light cue for 10 days/2hr/day. After 1 day or 30 days of withdrawal, rats received a pairing of sucrose with either lithium chloride or saline and two-days later, rats underwent cue-induced responding with no sucrose presentation. The rats that received the lithium chloride-sucrose pairing after 1 day of withdrawal did not show any effect (reduction or increase) on the incubation of craving whereas rats that received the pairing on day 30 of withdrawal had a significant reduction in cue-induced responding. The authors concluded that the incentive value of sucrose increased over the extended period of withdrawal and facilitated the transfer of lithium chloride aversion from the primary reward (sucrose) to the conditioned cue (Harkness et al., 2010).

\subsubsection{Mesolimbic DA system}

Time-dependent changes within the NAc have been highlighted in the incubation of craving (Glueck, Ginder, Hyde, North, \& Grimm, 2016; Grimm, Harkness, et al., 2011). These changes include alterations in overall neuron activity, DA receptor expression, AMPA receptors, and neuron structure (Christian et al., 2016; Dingess et al., 2017; Grimm et al., 2003; Koya et al., 2009b; Lee et al., 2013; Lu et al., 2005; Terrier et al., 2015). These time-dependent changes also differ between the NAcC and NAcSh subregions (Guillem et al., 2014). For example: After periods of abstinence from cocaine, neurons within the NAcC have been shown to have an increase in firing following 1 
month of withdrawal compared to earlier time points (Guillem et al., 2014). This increase in firing was not seen in the NAcSh (Guillem et al., 2014).

DA effects

The role of D1 receptors within the NAc in the incubation of sucrose craving was explored in a study by Grimm and colleagues (Grimm, Harkness, et al., 2011). The effect of systemic, and intra-accumbens injections (bilateral NAcC and NAcSh), of the D1 antagonist (SCH 23390) on cue-induced responding for sucrose at withdrawal day 1 and 30 was explored. Rats treated with saline responded more during day 30 of withdrawal than day 1. Systemic administration of SCH 23390 at low and moderate doses (1 and 5 $\mu \mathrm{g} / \mathrm{kg}$ ) reduced cue-induced responding for sucrose at day 1 of withdrawal but only the $25-\mu \mathrm{g}$ dose reduced responding for sucrose at day 30 of withdrawal. Injection into the NAcC and NAcSh of SCH $23390(0.3$ or $0.6 \mu \mathrm{g} / \mathrm{site})$ reduced cue-induced sucrose seeking at both day 1 and day 30 of withdrawal (Grimm, Harkness, et al., 2011). The results of this study indicate that D1 receptors in both the NAcC and NAcSh are involved in sucrose seeking (Grimm, Harkness, et al., 2011). Since $25 \mu \mathrm{g} / \mathrm{kg}$ was the only dose of SCH 23390 to reduce sucrose seeking following 30 days of abstinence, it suggests that there may be an increase in D1 receptors, hence a higher dose of the drug is necessary to abate sucrose seeking.

Glueck and colleagues (2017) investigated whether the ability of acute and chronic EE to reduce sucrose cue reactivity is mediated by DA receptors (Glueck et al., 2016). The study investigated whether D1 and/or D2 receptor agonism could reverse the EE effect on the incubation of sucrose craving. The D1 receptor agonist SKF 81297 (0, 0.3 , or $1 \mathrm{mg} / \mathrm{kg}$ ) or the D2 agonist quinpirole $(0,0.1$, or $0.3 \mathrm{mg} / \mathrm{kg})$ were administered systemically prior to a cue-reactivity test in animals that underwent acute (overnight before cue test) or chronic (29 day) EE during the forced abstinence period from sucrose. Following 30 days of abstinence, SKF 81297 administration increased cue reactivity in acute $\mathrm{EE}$, chronic $\mathrm{EE}$, and control rats and quinpirole resulted in a similar cue reactivity for both control and EE rats. This suggests that following 30 days of abstinence D1 receptors may be important for cue reactivity (Glueck et al., 2016).

Glutamate effects (AMPA)

Synaptic changes, such as increased CP-AMPA receptors, have been observed within the NAc (Conrad et al., 2008; Loweth, Tseng, \& Wolf, 2014; Wolf \& Tseng, 2012) following a period of withdrawal from cocaine and sucrose (Conrad et al., 2008; Counotte et al., 2014; Wolf and Tseng, 2012). A study by Conrad and colleagues (2008) investigated incubation of cocaine craving and determined an increase in nose pokes on withdrawal day 45 vs withdrawal day one during an extinction test, where nose pokes resulted in cue delivery but not cocaine (Conrad et al., 2008). On day 45, there was an associated increase in CP-AMPA receptors in the NAc. The authors hypothesized that the increase in CP-AMPA receptors results in increased NAc reactivity to cocaine-related cues, which was associated with drug craving and relapse (Conrad et al., 2008). An injection of the CP-AMPA antagonist 1-naphthyl acetyl spermine (NASPM) into the NAc prior to cue-induced cocaine seeking resulted in decreased responding on day 45 but 
not day 1 of withdrawal, providing further support for the contribution of CP-AMPA receptors to the incubation of craving (Conrad et al., 2008).

Alterations in AMPA receptors have also been shown following incubation of methamphetamine and sucrose craving (Counotte et al., 2014; Scheyer et al., 2016). Scheyer and colleagues (2016) used whole-cell patch recordings and intra-NAcC injection of NASPM to characterize AMPA receptor activity following 45 days of withdrawal from methamphetamine (Scheyer et al., 2016). CP-AMPA receptor accumulation within the NAcC was at its highest approximately 1 week after withdrawal, at the same time period intra-NAcC NASPM administration reduced the expression of the incubation of craving (Scheyer et al., 2016). These results differ from cocaine, which can take between 25 and 35 days following cessation to show an increase in CP-AMPA receptors (Wolf \& Tseng, 2012).

Time-dependent changes in AMPA/NMDA ratios have been demonstrated following the incubation of sucrose craving in both adolescent and adult rats (Counotte et al., 2014). Following 21 days of abstinence, but not 1-3, both adolescent and adult rats have been shown to have a lower AMPA/NMDA receptor ratio within the NAc. The authors suggested that these data are in agreement that there is a limited change in CPAMPA receptors following 21 days of withdrawal (Counotte et al., 2014). Testing at later time points may reveal a change in CP-AMPA receptors similar to that seen with cocaine, but this has yet to be seen.

Immediate early Genes

Grimm and colleagues (2016) investigated Fos levels in numerous brain regions, including the NAc, following 1 and 30 days of forced abstinence from sucrose (Grimm et al., 2016). The effect of EE on sucrose seeking and associated Fos levels was also explored. In both the NAcC and NAcSh, Fos positive cells were increased following 30 days of forced abstinence from sucrose compared to 1 day. An interaction effect between $\mathrm{EE}$ and incubation was also observed in the NAcC and NAcSh where EE was effective at reducing Fos positive cells after 30 days of forced abstinence (Grimm et al., 2016).

\section{Structural Plasticity}

Changes in synaptic spine density have been observed in the NAc following forced abstinence from both cocaine and high fat (60\%) food (Christian et al., 2016; Dingess et al., 2017b). Spine density and morphology were investigated in the NAcC following 14, 25, 36, and 60 days of withdrawal from cocaine (Christian et al., 2016). Rats showed no difference in spine density or morphology in the NAcC following 14 or 25 days of withdrawal compared to saline controls. Following 36 days of withdrawal, the same time frame that elevated CP-AMPA receptor levels have been detected, the cocaine group exhibited an increase in thin spine density. However, by day 60, the density of thin spines in the cocaine group was lower compared to the saline group even though CPAMPA receptor levels have been shown to remain high at withdrawal day 60 (Christian et al., 2016). These results show alterations in spine density over the course of withdrawal. In terms of food, high fat food has been shown to result in an increase in 
mushroom type spines following 30 days of withdrawal in the NAcC but not the NAcSh (Dingess et al., 2017b). Normal chow results in an increase in spines within both the NAcC and NAcSh following 30 days of forced abstinence (Dingess et al., 2017b).

\subsection{Objectives and Hypotheses}

Incubation of craving has been studied with respect to cocaine, amphetamine, and sucrose, but has yet to be extensively studied with respect to palatable food. For example, chocolate is one of the most palatable foods to humans and is a food that people on diets attempt to avoid. Since relapse from diets is around $80 \%$, understanding craving for palatable food could contribute knowledge surrounding the obesity epidemic.

Relapse from dieting, even after long-term success, may be the result of long-term neural changes, such as those seen in drug addiction (c-Fos, DeltaFosB, CP-AMPA, Dendritic spines).

Forced abstinence from palatable foods has not been extensively assessed for incubation of craving, when it has been measured there have been several discrepancies in the outcomes. Neural correlates of palatable food craving also need to be explored to determine similarities between palatable food and drug craving. This may elucidate potential treatment targets for food cravings. Assessing incubation of palatable food craving using different animal models is also necessary to determine if the incubation effect is behaviourally specific. It is possible that an incubation response is the result of the conditioning procedure (bar-press paired with light, plus a time-out) and does not expand to include other learning scenarios (operant-no time-out, Classical conditioning, CPP). It is hypothesized that following self-administration of food reward rats will display an incubation of craving response. This hypothesis is based on the consistent incubation of craving response displayed following self-administration of drug and natural rewards in the current literature (Grimm et al., 2005; Krasnova et al., 2014d; Lu et al., 2004). Rewards that are administered by the experimenter have been less consistent in producing an incubation of craving response, thus experimenter reward delivery may not produce an incubation response (Li et al., 2008; Sun et al., 2017). Changes, such as c-Fos, FosB, and dendritic spine, should increase/decrease with the magnitude of reward seeking behavior. The present thesis explored the incubation of craving for food reward and associated NAc changes in an attempt to elucidate the type of behavioral learning and food reward that is necessary to produce an incubation response. 


\section{Chapter 2}

\section{Comparison of the Time-dependent Changes in Immediate Early Gene Labeling and Spine Density Following Abstinence from Contingent or Non- contingent Chocolate Pellet Delivery}

Erin W. Noye Tuplin, Savannah H. M. Lightfoot, and Matthew R. Holahan

Frontiers in Behavioral Neuroscience, 2018

In Press. doi: https://doi.org/10.3389/fnbeh.2018.00144

Department of Neuroscience, Carleton University

Keywords: FosB, c-Fos, craving, dendritic spines, food reward, operant conditioning, classical conditioning

\subsection{Abstract}

Rationale: Incubation of craving is a phenomenon whereby responding for cues associated with a reward increases over extended periods of abstinence. Both contingent and non-contingent behavioral designs have been used to study the incubation of craving phenomenon with differing results. The present study directly compares behavioral and neural changes following contingent or non-contingent administration of chocolate flavored pellets.

Objective: The current study examined whether an incubation of craving response would be observed at the behavioral and neural levels following delays of abstinence from chocolate pellets in a contingent or non-contingent reinforcement design. 
Methods: Rats were trained for 10 days to bar press for chocolate pellets (contingent) or received chocolate pellets in a non-contingent design (classical conditioning). Groups were then subjected to abstinence from the reward for $24 \mathrm{hrs.} 7,14$ or 28 days at which point they were tested for responding for reward associated cues. Following the test, brains from all rats were processed and assessed for c-Fos and FosB labeling as well as dendritic spine density in the nucleus accumbens (NAc).

Results: Behavioral measures during the test (lever presses, food hopper entries and locomotor activity) revealed similar behavioral outcomes across all delays indicating the lack of an incubation of craving response on both the contingent and non-contingent designs. Overall, labeling of c-Fos in the NAc was lower for the non-contingent group compared to the operant-trained and food restricted control. Compared to the operanttrained and non-trained control groups, a significantly reduced FosB labeling was noted in the NAc of the classically conditioned groups across all abstinence periods. Spine density in the NAc was elevated in both the classically and operant conditioned compared to the food-restricted, non-trained controls.

Conclusions: Chocolate pellet reward did not result in incubation of craving but did produce behavioral learning that was associated with increased spine density. This suggests that chocolate pellet administration results in long-term structural and functional changes that are present for at least 28 days following abstinence. Contingent and noncontingent administration resulted in differential immediate early gene labeling in the NAc, but the functional significance of this has yet to be elucidated.

\subsection{Introduction}

Relapse following a period of withdrawal from an addictive substance is often associated with craving, a poorly understood phenomenon that precedes drug-seeking behaviors (Sayette, 2016; Tiffany and Wray, 2012). Craving can be triggered by cues previously associated with drug-taking (Veilleux and Skinner, 2015). These cues include the drug itself as well as sights, sounds, odors, and locations associated with the drugtaking experience (Harkness et al., 2010; Obrien et al., 1992). Exposure to these drugrelated cues may evoke physiological changes that result in craving and contribute to subsequent drug seeking and taking behaviors (Sayette, 2016; Veilleux and Skinner, 2015). Craving may continue even after physiological withdrawal symptoms have subsided (Grimm et al., 2002, 2016; Lu et al., 2004; Pickens et al., 2011). Animal models of drug craving suggest that the onset of craving may be delayed, and even enhanced, following a period of abstinence from a substance. These time-dependent changes in craving following a period of abstinence are termed "incubation of craving" (Grimm et al., 2011a; Hale et al., 2001; Lu et al., 2004).

The majority of studies investigating the incubation of craving focus on selfadministration (contingent) of a rewarding substance in the presence of discrete environmental cues. In self-administration models, craving is defined as increased responding on the correct lever where depressing the lever results in the discrete cue(s) previously paired with the reward. Studies involving the self-administration of cocaine have shown that following a period of abstinence, rats have increased cue-induced 
responding on day 30 compared to day 1 of withdrawal (Calu et al., 2007; Grimm et al., 2001, 2002; Halbout et al., 2014; Lu et al., 2004). An incubation effect has also been observed following self-administration of methamphetamine (Li et al., 2015b, 2015a; Shepard et al., 2004) nicotine (Abdolahi et al., 2010; Funk et al., 2016) heroin (Shalev et al., 2001), and alcohol (Bienkowski et al., 2004).

Incubation of craving for reward has also been studied using non-contingent models of substance administration, but when non-contingent models of administration are utilized, the effect is less consistent. Diehl and colleagues (2013) used a model of cue-induced conditioned activity, where discrete cues were paired with cocaine administration, to determine if the locomotor responses to cocaine sensitized during withdrawal (Diehl et al., 2013b). Rats had consistent locomotor activity following 3, 14, and 28 days of withdrawal from cocaine in this non-contingent model (Diehl et al., 2013b). Conditioned place preference (CPP) models of incubation of craving have revealed variable results. Sun and colleagues (2017) demonstrated a decrease in CPP scores following forced abstinence from morphine whereas Li and colleagues (2008) found that CPP for morphine increased over a 14 day abstinence period and Lubbers and colleagues (2016) demonstrated an increase in CPP scores following 3 weeks of withdrawal from cocaine (Li et al., 2008; Lubbers et al., 2015; Sun et al., 2017). In the case where CPP was not noted, animals displayed a progressive increase in side entries following abstinence from morphine suggesting enhanced drug seeking behavior (Sun et al., 2017). The use of self-administered or experimenter administered procedures may be an important factor in observing the occurrence of an incubation of craving effect. These procedural variations may also differentially engage brain regions involved in reward.

The nucleus accumbens (NAc) has been implicated in the rewarding aspects of a variety of substances and compulsive behaviors related to obtaining reward (Adinoff, 2004; Chaudhri et al., 2010; Chiara and Di Chiara, 2002; Grimm et al., 2011b; Nestler, 2012; Quintero, 2013; Wolf and Tseng, 2012). Specifically, the NAcSh has been shown to play a role in incentive arousal for addictive substances (Di Chiara et al., 2004). Selfadministration and non-contingent administration of drug rewards has been associated with changes in immediate early genes (Larson et al., 2010; Li et al., 2015a; Renthal et al., 2008) and dendritic spine density (Christian et al., 2016) in the NAc core (NAcC) and shell (NAcSh). Cocaine that is self-administered (lever pressing) is associated with increased c-Fos labeling in the NAcSh but not in the NAcC (Larson et al., 2010). Experimenter administered amphetamine is also associated with increased c-Fos labeling but labeling decreased with repeated administration (Renthal et al., 2008). Chronic cocaine, both self-administered and experimenter administered, is associated with increased DeltaFosB labeling in the NAcSh (Larson et al., 2010). In the NAcC, both acute and chronic experimenter administered cocaine is associated with an increase in DeltaFosB (Larson et al., 2010). Chronic self-administered or experimenter administered amphetamine or cocaine is associated with increased spine density on medium spiny neurons (MSN)s in the NAcSh and NAcC (Robinson and Kolb, 1999, 2004). The increase in spine density persists months after amphetamine discontinuation and at least a 
month after cocaine discontinuation (Robinson and Kolb, 2004). Specific to the incubation of craving, rats trained to self-administer cocaine showed increased dendritic spine density in the NAcC following 36 days of withdrawal compared to 1 day (Christian et al., 2016).

Incubation of craving has been demonstrated for other rewarding stimuli such as sucrose (Grimm et al., 2005; Lu et al., 2007) and palatable foods (Darling et al., 2016; Krasnova et al., 2014a) and is also is associated with changes in NAc immediate early genes and synaptic spines (Dingess et al., 2017a; Grimm et al., 2016). Incubation of craving for sucrose is associated with elevated c-Fos-positive cells following 30 days of forced abstinence compared to 1 day in both the NAcC and NAcSh (Grimm et al., 2016). Self-administration of food reward has been shown to increase dendritic spine density within the NAcSh (Guegan et al., 2013; Mancino et al., 2016). Both high fat food and chow results in an increase in spines following 30 days of withdrawal (Dingess et al., 2017a).

There are currently a dearth of studies exploring the incubation of craving for food reward comparing self-administration (contingent) and experimenter administration (non-contingent) and the associated NAc changes. Relapse from diets is around $80 \%$ (Brandon et al., 2007), thus understanding craving for food reward could contribute knowledge to the rising obesity epidemic. Relapse following dieting, even after longterm success, may be the result of long-term neural changes, such as those seen in drug addiction. The present study explored the incubation of craving for chocolate-flavored pellets using both operant (contingent) and classical conditioning (non-contingent) designs.

The operant (contingent) design followed an FR2 schedule of reward with delivery of a chocolate flavored-pellet coinciding with a discrete cue for 5 seconds. Studies within our laboratory have utilized an FR2 responding for chocolate-flavored pellets during operant acquisition. These studies resulted in robust responding that reached a peak by day 5 of acquisition (Davis-MacNevin et al., 2013; Holahan et al., 2010, 2012; Tuplin et al., 2015). The classical conditioning (non-contingent) design utilized a variable interval schedule of non-contingent chocolate delivery, which has yet to be studied with respect to the incubation of craving. The classical conditioning design paired a discrete cue with delivery of a chocolate flavored pellet. During testing, levers were introduced into the chamber and rats had the opportunity to bar press for the cue.

Markers of neuronal activation, FosB and c-Fos, were quantified in the NAcC and NAcSh. FosB was chosen as it is a common marker of neuron activity and addiction (Nestler, 2004, 2008, 2015) but has yet to be studied with respect to incubation of craving. The c-Fos protein was chosen because it represents an index of neuronal activity in the NAc during the behavioral testing procedure. Quantification of dendritic spines within the NAcSh was chosen because spines tend to change over time following learning and withdrawal and represent a form of structural plasticity. 


\subsection{Methods}

\subsubsection{Subjects}

A total of 63 male Long Evans rats (250-275 grams) were obtained from Charles River, Quebec. Rats were housed individually in clear plastic cages $(25 \times 20 \times 45 \mathrm{~cm})$, given water ad libitum under a 12-hour light/dark cycle (lights on at 8:00 am), and tested during the light phase. Previous operant studies within our laboratory have been undertaken during the light phase, which did not interfere with adequate acquisition of the task (Davis-MacNevin et al., 2013; Holahan et al., 2010; Tuplin et al., 2015). Food was restricted to $90 \%$ baseline, which was maintained until the end point of the studies. Prior to behavioral testing, rats were handled for 5 minutes daily and given 10, 45-mg chocolate pellets to acclimate them to handling and the reward. Principles of laboratory animal care were followed and all procedures were conducted in accordance with the Canadian Council on Animal Care. The Carleton University Animal Care Committee approved the protocol.

\subsubsection{Operant acquisition and responding for cues}

A total of 35 rats underwent operant acquisition training, which occurred over 10 days. During acquisition sessions, rats were placed in the operant chambers (Coulburn Instruments, Whitehall, PA; $30.5 \mathrm{~W}$ x $25.5 \mathrm{D}$ x $30.5 \mathrm{H} \mathrm{cm}$ ) for $1 \mathrm{hr}$., where every second response (FR2 schedule) on the correct lever resulted in the delivery of a $45 \mathrm{mg}$ chocolate pellet (BioServe, Flemington, NJ). Once the correct lever had been depressed twice (FR2), the house light extinguished and panel lights above the lever changed from red to green for 5 seconds. This was accompanied by the pellet dispenser releasing one 45-mg chocolate pellet into the food hopper. The incorrect lever had no programmed consequences. Both correct and incorrect presses, nose pokes into the food hopper, and locomotor activity were recorded during the sessions. Nose pokes into the food hopper were assessed with a photocell beam-break detector that contained an infrared LED source and photodetector on the sides of the food hopper. Locomotor activity was assessed with a ceiling mounted activity monitor tuned to be sensitive to the infrared signals emitted by a rat. Movement units (brief pulses representing the minimum resolution of detection) were used to provide a measure of locomotor activity.

Following operant acquisition, rats were assigned to one of four abstinence groups and tested at different time delays in the absence of food reward. Rats were matched based on total correct presses during day 10 of acquisition to ensure all groups (abstinence periods) had obtained a similar level of acquisition. Rats were assigned to groups with the following periods of forced abstinence from chocolate: $24 \mathrm{hrs}$. (n=9), 7 days $(n=9), 14$ days $(n=8)$, or 28 days $(n=9)$. Each rat was tested in the same operant chamber where operant acquisition training occurred. The testing session was $1 \mathrm{hr}$. and bar pressing no longer resulted in food reward but the discrete cues were present (the house light extinguished and the panel lights changed from red to green for 5 seconds). 
Correct presses, incorrect presses, nose pokes into the food hopper, and locomotor activity were recorded at 10 minute intervals.

\subsubsection{Classical Conditioning and responding for cues}

A total of 26 rats underwent classical conditioning over 10 days. During the conditioning sessions, rats were placed into the operant chambers (Coulburn Instruments, Whitehall, PA; $30.5 \mathrm{~W}$ x $25.5 \mathrm{D}$ x $30.5 \mathrm{H} \mathrm{cm}$ ) for $1 \mathrm{hr}$. where one $45-\mathrm{mg}$ chocolate pellet was dispensed on a variable interval 30 -second schedule. The house light extinguishing and the panel lights changing from red to green for 5 seconds was accompanied by the presentation of the $45 \mathrm{mg}$ chocolate pellet. Nose pokes into the food hopper and locomotor activity were recorded during each 1-hr conditioning session.

Following conditioning, rats were assigned to one of four abstinence period groups $(24$ hrs. $(n=6), 7$ days $(n=6), 14$ days $(n=6)$, or 28 days $(n=6))$ and tested in a responding for cues behavioral design. Rats were matched based on total nose pokes on day 10 of conditioning to ensure all groups (abstinence periods) had obtained a similar level of conditioning. Testing occurred in the same chambers as training. Two levers were introduced into the operant chambers and a correct lever press resulted in the presentation of the conditioning cues (house light extinguishing and panel lights changing from red to green for 5 seconds) and an incorrect lever press had no programmed consequences. Rats were tested for $1 \mathrm{hr}$. and correct presses, incorrect presses, nose pokes into the food hopper, and locomotor activity were recorded at 10-minute intervals.

\subsubsection{Controls}

There was a second treatment where rats were food restricted but did not undergo behavioral training or testing ( $24 \mathrm{hr} .: \mathrm{n}=3,7$ day: $n=3,14$ day: $n=3,28$ day: $n=3$ ). These rats underwent food restriction for the same period of time as rats that underwent behavioral testing. Food restriction for the control rats continued throughout the 10 days that rats were behaviorally trained. The control rats were continually food restricted during the abstinence periods so that total food restriction was equal to the behaviorally trained rats. Control rats were euthanized at specific abstinence periods to determine if food restriction on its own affected immunohistochemical labeling or dendritic spine density.

\subsubsection{Immunohistochemistry}

Ninety minutes following testing, rats were given an overdose of sodium pentobarbital and perfused with saline. Control rats were euthanized at the same time of day as behaviorally tested rats. One hemisphere was immersion fixed in $4 \%$ paraformaldehyde/0.01M phosphate buffer solution ( $\mathrm{pH} 7.4$ ). The other hemisphere was placed in a Golgi Fix solution. Following 24 hrs. the paraformaldehyde-immersed tissue was cryopreserved via immersion in a $30 \%$ sucrose $/ 0.1 \mathrm{M}$ phosphate buffer solution for a 
minimum of 4 days. The tissue was then sectioned on a cryostat at $60 \mu \mathrm{m}$ and placed in a $0.1 \%$ sodium azide/0.1 M phosphate buffer solution ( $\mathrm{pH} 7.4$ ).

FosB and c-Fos

Two sections with the NAc from each rat were chosen for FosB and two other sections were chosen for c-Fos immunohistochemical staining. Sections were chosen from two coronal levels per rat, bregma $+2.0 \mathrm{~mm}$ and $+1.7 \mathrm{~mm}$ (Figure 2.1), which were averaged for analysis. This region was chosen as it has been shown to be associated with feeding behavior (Stratford and Kelley, 1997). Sections underwent three, 5-minute washes in $0.1 \mathrm{M}$ phosphate buffered saline with $0.1 \%$ Triton X (PBS-TX) followed by incubation in a blocking solution of $0.3 \%$ hydrogen peroxide diluted with PBS-TX for 15-minutes. Sections underwent three more 5-minute washes in PBS-TX followed by incubation in animal free blocker (AFB; Vector Laboratories) diluted to 1x with PBS-TX from a $5 \mathrm{X}$ concentrated solution. Sections were incubated overnight at room temperature in the primary antibody (1:5000 anti-FosB host: mouse Cat \# ab11959; Abcam or 1:5000 anti-c-Fos host: rabbit Cat \# 226003; Synaptic Systems). The following day, tissue underwent three 10-minute washes in PBS-TX followed by incubation in the secondary antibody (FosB - 1:1000 goat anti-mouse IgG: Vector; c-Fos - 1:500 goat anti-rabbit IgG: Vector) for $1 \mathrm{hr}$. at room temperature. Next, the tissue underwent three 10-minute washes in PBS followed by immersion in Avidin-Biotin Complex (ABC) for $1 \mathrm{hr}$. Following $\mathrm{ABC}$, the tissue underwent three, 5-minute washes in PBS and was developed in 3,3'-diaminobenzidine (DAB) for approximately 5 minutes. Following development, the tissue was washed twice in PBS for 5 minutes then mounted onto slides, dehydrated, and coverslipped.

\section{Immunohistochemistry quantification}

A counting method based on unbiased stereology procedures using the optical fractionator method was used to estimate the number of FosB and c-Fos labeled cells in the NAcC and NAcSh subregions (see Figure 2.1A for delineation of these regions). The NAc was traced at 2x magnification using an Olympus BX51 bright field microscope with a motorized stage (Olympus Canada, Markham, ON) and c-Fos (examples of staining in Figure 2.1B) and FosB (see Figure 2.1C for examples of staining) labeled cells were quantified using a 60x magnification lens (oil immersion, NA 135). Counting was performed using sampling parameters sufficient to produce a Gunderson's coefficient of error $(\mathrm{GCE}, \mathrm{m}=1)$ of 0.12 or less. Stereo Investigator software used planar and depth information for each counted cell to calculate the volume for the digitally traced region of interest. Counting parameters were set to a counting frame of $60 \times 60 \mu \mathrm{m}^{2}$ and a dissector height of $15 \mu \mathrm{m}$ between the top and bottom guard zones. Estimated population number using weighted section thickness was recorded for each animal. The estimated cell count from two sections for each animal was averaged to give one datum point. Quantification is represented as an estimated total per mean measured thickness per $\mathrm{mm}^{3}$ 
to allow for comparisons across brain sections. A minimum of three animals per group were included in the analyses.

\subsubsection{Golgi staining}

The Golgi fixative consisted of three solutions that were prepared separately and combined in the final step of the process. Solution A required heating $750 \mathrm{ml}$ of distilled water to 90 degrees Celsius prior to the addition of 37.5 grams $(\mathrm{g})$ of mercuric chloride $(\mathrm{Hg})$, the solution was then allowed to cool. Solution B required the heating of $750 \mathrm{ml}$ of $\mathrm{dH} 20$ to 60 degrees Celsius followed by the addition of $37.5 \mathrm{~g}$ of potassium dichromate (K2Cr2O7), this solution was then allowed to cool. Solution $\mathrm{C}$ was made by dissolving $30 \mathrm{~g}$ of potassium chromate $(\mathrm{K} 2 \mathrm{Cr} 2 \mathrm{O} 4)$ in 750 of $\mathrm{dH}_{2} \mathrm{O}$. Solutions $\mathrm{A}, \mathrm{B}$, and $\mathrm{C}$ were then combined in a new flask and $1500 \mathrm{ml}$ of room temperature $\mathrm{dH}_{2} \mathrm{O}$ was added. The solution was stored in a glass jar and covered in tinfoil for a least 6 days prior to use.

Following 14 days of incubation in the Golgi fixative, whole brains underwent a series of $\mathrm{dH}_{2} \mathrm{O}$ and sucrose washes in preparation for tissue sectioning and development. Initially, brains were immersed in $\mathrm{dH}_{2} \mathrm{O}$ for $4 \mathrm{hrs}$. then immersed in fresh $\mathrm{dH}_{2} \mathrm{O}$ for another $3 \mathrm{hrs}$. followed by fresh $\mathrm{dH}_{2} \mathrm{O}$ overnight. On the second day of washes, brains were immersed in $10 \%$ sucrose for $8 \mathrm{hrs}$. followed by $20 \%$ sucrose overnight. On the third day, brains were immersed in $30 \%$ sucrose for a minimum of 4 days prior to sectioning on a Vibratome.

A humidified box with a plastic grate was created $24 \mathrm{hrs}$. prior to sectioning to prevent the tissue from drying when it was slide mounted. Tissue was sectioned on a Vibratome at $200 \mu \mathrm{m}$ and floated in a 30\% sucrose solution prior to mounting. Twenty sections were taken from each brain through the NAc and placed immediately onto double dipped (2\%) gelatinized slides. Slides were then placed in the humidified box in a dark cupboard for no longer than 24 hours.

A desiccated box was created 24 hrs. prior to development by placing Drierite pellets in the bottom of a plastic box that contained a plastic grate. Golgi staining was developed by immersing tissue mounted slides in several solutions, beginning with a 1 minute rinse in $\mathrm{dH}_{2} \mathrm{O}$. Next, slides were submerged in $28 \%$ ammonium hydroxide for 40 minutes and then $\mathrm{dH}_{2} \mathrm{O}$ for one minute. Slides were then submerged in Kodak Film Fix A (diluted 1:1 with $\mathrm{dH}_{2} \mathrm{O}$ ) for 40 minutes where the solution was kept protected from light. Next, slides were washed twice in $\mathrm{dH}_{2} \mathrm{O}$ prior to dehydration. For dehydration, slides were submerged in 50\% ethyl alcohol (ETOH) for 1 minute, $75 \%$ ETOH for 1 minute, and $95 \% \mathrm{ETOH}$ for 1 minute. The remaining solutions were desiccated with molecular sieve for at least $24 \mathrm{hrs}$. prior to use. Slides underwent three 5-minute washes in $100 \%$ desiccated ETOH following by a 10 minute wash in a desiccated solution of $33 \%$ ETOH, 33\% Clearene, and 33\% chloroform. The final wash consisted of slides being placed in desiccated Clearene for 15 minutes. Slides were then coverslipped with Permount and placed in a desiccated box for a minimum of 3 days to allow the Permount to harden. 


\section{Quantification of dendritic spines}

For each of the three behavioral groups (operant, classical, control), three MSNs within the NAcSh per rat, with a minimum of 3 rats per abstinence period were analyzed. This resulted in a minimum of 9 neurons per abstinence period within each of the behavioral groups. The NAcSh was constricted in the anterior-posterior plane from approximately +1.7 to $+3.2 \mathrm{~mm}$ from bregma according to Paxinos and Watson (1998). Neurons were picked at random but had to meet specific criteria to be selected. Neurons had to be entirely impregnated, staining had to be uniform and complete, the cell body had to be within the $200 \mu \mathrm{m}$ section depth, and the neuron had to be relatively isolated from surrounding neurons. Neurons were reconstructed at 100x magnification using Neurolucida software (MicroBrightField, Williston, VT, USA). The cell body was traced first, followed by a minimum of three primary dendrites and subsequent branches, in their entirety, and all visible spines were marked. For each neuron, the total number of spines were analyzed and divided by total dendritic length to obtain the spine density of each neuron.

\subsubsection{Statistical analysis}

Behavioral data from each experimental design (contingent or non-contingent) were analyzed separately. Acquisition data were analyzed with a two-way, repeated measures ANOVA using abstinence period as the between factor and total correct presses (operant acquisition) or total nose pokes (classical conditioning) per day as the repeated measure. The testing data were analyzed using a two-way, repeated measures ANOVA using abstinence period as the between factor and time as the repeated measure. Correct presses, incorrect presses, nose pokes into the food hopper, and locomotor activity were analyzed separately. If ANOVAs were significant, Tukey post-hoc comparisons were performed. Alpha was set at 0.05 for significance.

FosB and c-Fos estimated cell counts were analyzed with fixed factor ANOVAs with abstinence period and behavioral group (operant, classical, control) as independent variables. Counts from the NAcC and NAcSh were analyzed separately. Significant interactions were analyzed with one-way ANOVAs. One-way ANOVAs were also used to determine differences in abstinence periods within individual behavioral groups. Tukey's post-hoc analysis was used if one-way ANOVAs were significant. Alpha was set at 0.05 for significance.

Dendritic spine density analysis within the NAcSh included measurements from three neurons per rat/per brain being averaged to get one datum point per rat. Spine density was analyzed using a factorial ANOVA with abstinence period and behavioral group as the independent variables. Significant interactions were analyzed with one-way ANOVAs. One-way ANOVAs were also used to determine differences in abstinence periods within individual behavioral groups. Tukey's post-hoc analysis was used if oneway ANOVAs were significant. Alpha was set at 0.05 for significance. 


\subsection{Results}

\subsubsection{Operant acquisition and responding for cues}

Rats were matched and assigned to one of four abstinence periods based on correct lever presses after day 10 of acquisition training (Figure 2.2A). A two-way repeated measures ANOVA on the average number of correct presses per day as the repeated measure and abstinence period as the between factor revealed a main effect of day, $(\mathrm{F}(9$, $27)=62.030, \mathrm{p}<0.001)$ but no main effect of abstinence period, $(\mathrm{F}(3,31)=0.265, \mathrm{p}>$ $0.05)$, and no interaction, $(F(27,279)=0.769, p>0.05)$. Nose pokes into the food hopper and locomotor activity were also assessed to ensure there were no differences between groups after the last day of acquisition. A two-way repeated measures ANOVA on the average number of nose pokes into the food hopper (Figure 2.2B) per day as the repeated measure and abstinence period as the between factor revealed a main effect of day, $(\mathrm{F}(9,27)=9.327, \mathrm{p}<0.001)$ but no main effect of abstinence period, $(\mathrm{F}(3,31)=0.117, \mathrm{p}$ $>0.05)$, and no interaction, $(\mathrm{F}(27,279)=0.645, \mathrm{p}>0.05)$. A two-way repeated measures ANOVA on the average locomotor activity (figure 2.2C) per day as the repeated measure and abstinence period as the between factor revealed a main effect of day, $(\mathrm{F}(9,27)=$ $5.540, \mathrm{p}<0.001)$ but no main effect of abstinence period. $(\mathrm{F}(3,31)=1.272, \mathrm{p}>0.05)$ and no interaction, $(\mathrm{F}(27,279)=1.498, \mathrm{p}>0.05$.

Rats were given one, 60 min extinction test 24 hrs., 7 days, 14 days, or 28 days after the last day of operant acquisition. A two-way repeated measures ANOVA on the number of correct lever presses (Figure 2.2D) with time (10-minute intervals) as the repeated measure and abstinence period as the between factor revealed a main effect of time, $(\mathrm{F}(5,15)=34.009, \mathrm{p}<0.01)$ but no main effect of abstinence period, $(\mathrm{F}(3,31)=$ $1.131, \mathrm{p}>0.05)$ and no interaction, $(\mathrm{F}(15,155)=1.125, \mathrm{p}>0.05)$.

A two-way repeated measures ANOVA on the number of nose pokes into the food hopper (Figure 2.2E) with time (10-minute intervals) as the repeated measure and abstinence period as the between factor revealed a main effect of time, $(F(5,15)=9.612$, $\mathrm{p}<0.001)$ but no main effect of abstinence period, $(\mathrm{F}(3,31)=0.588, \mathrm{p}>0.05)$ and no interaction, $(\mathrm{F}(15,155)=0.667, \mathrm{p}>0.05)$.

A two-way repeated measures ANOVA on locomotor activity (Figure 2.2F) with time (10-minute intervals) as the repeated measure and abstinence period as the between factor revealed a main effect of time, $(\mathrm{F}(5,15)=81.719, \mathrm{p}<0.001)$ and a main effect of abstinence period, $(\mathrm{F}(3,31)=3.152, \mathrm{p}<0.05)$ but no interaction, $(\mathrm{F}(15,155)=1.555, \mathrm{p}>$ 0.05). Tukey post-hoc analysis on the main effect of abstinence period revealed that the 14-day abstinence period group had significantly higher locomotor activity than the 28day abstinence period group, $(\mathrm{p}<0.05)$.

\subsubsection{Classical conditioning and responding for cues}

Rats were matched and assigned to one of four abstinence periods based on nose pokes into the food hopper after day 10 of conditioning (Figure 2.3A). A two-way 
repeated measures ANOVA on the average number of nose pokes per day as the repeated measure and abstinence period as the between factor revealed a main effect of day, $(\mathrm{F}(9,27)=4.222, \mathrm{p}<0.001)$ but no main effect of abstinence period, $(\mathrm{F}(3,21)=0.716, \mathrm{p}$ $>0.05)$, and no interaction, $(\mathrm{F}(27,189)=0.386, \mathrm{p}>0.05)$. A two-way repeated measures ANOVA with locomotor activity per day as the repeated measure and abstinence period as the between factor revealed a main effect of day, $(F(9,27)=10.383, p<0.001)$ but no main effect of abstinence period, $(\mathrm{F}(3,21)=0.897, \mathrm{p}>0.05)$, and no interaction, $(\mathrm{F}(27,189)=0.911, \mathrm{p}>0.05)$.

Rats in the classical conditioning groups were given an operant transfer test 24 hrs., 7 days, 14 days, or 28 days after the last day of acquisition. For the test, two levers were introduced into the chambers and pressing on one (correct) resulted in presentation of the cues that were paired with reward during training and pressing on the other (incorrect) had no programmed consequences A two-way repeated measures ANOVA on the number of correct presses (Figure 2.3C) with time (10-minute intervals) as the repeated measure and abstinence period as the between factor revealed a main effect of time, $(\mathrm{F}(5,15)=7.082 \mathrm{p}<0.001)$, but no effect of abstinence period, $(\mathrm{F}(3,20)=0.818, \mathrm{p}$ $>0.05)$ and no interaction, $(\mathrm{F}(15,100)=0.880, \mathrm{p}>0.05)$.

A two-way repeated measures ANOVA on the number of nose pokes into the food hopper (Figure 2.3D) with time (10-minute intervals) as the repeated measure and abstinence period as the between factor revealed a main effect of time, $(\mathrm{F}(5,15)=11.494$, $\mathrm{p}<0.001)$ and a significant interaction, $(\mathrm{F}(15,100)=2.723, \mathrm{p}<0.01)$ but no main effect of abstinence period, $(\mathrm{F}(3,20)=2.766, \mathrm{p}>0.05)$. One-way ANOVAs explored differences in nose pokes between abstinence periods at each 10-minute time point (Figure 2.3D). A main effect of abstinence period was revealed at twenty minutes, $(\mathrm{F}(3,20)=3.379, \mathrm{p}<0.05)$, forty minutes, $(\mathrm{F}(3,20)=3.089, \mathrm{p}<0.05)$ and fifty minutes, $(\mathrm{F}(3,20)=5.439, \mathrm{p}<0.01)$ during the test. Tukey post-hoc comparisons between abstinence periods at these time points revealed that during the twenty-minute interval, the 7-day abstinence period group showed significantly more nose pokes than the 28-day period group $(\mathrm{p}<0.05)$. During the fifty-minute interval, the 14-day abstinence period group showed significantly more nose pokes than the $24 \mathrm{hr}$. and 28-day period groups (p $<0.05)$.

A two-way repeated measures ANOVA on locomotor activity (Figure 2.3E) with time (10-minute interval) as the repeated measure and abstinence period as the between factor revealed a main effect of time, $(\mathrm{F}(5,15)=38.488), \mathrm{p}<0.001$ but no main effect of group, $(\mathrm{F}(3,20)=0.924, \mathrm{p}>0.05)$ and no interaction, $(\mathrm{F}(15,100)=0.961, \mathrm{p}>0.05)$.

\subsection{3 c-Fos}

A fixed factor ANOVA on the estimated density of c-Fos labeled cells in the NAcSh and NAcC (Figure 2.4) revealed a main effect of abstinence period in the NAcSh, $(\mathrm{F}(3,31)=2.920, \mathrm{p}<0.05)$ but not in the NAcC, $(\mathrm{F}(3,31)=1.735, \mathrm{p}>0.05)$ and no interaction between behavioral condition and abstinence period in either region (NAcSh: 
$(\mathrm{F}(6,31)=0.552, \mathrm{p}>0.05)$; NAcC: $(\mathrm{F}(6,31)=1.080, \mathrm{p}>0.05)$. There was a main effect of behavioral condition in both regions (NAcSh: $(\mathrm{F}(2,31)=8.373, \mathrm{p}<0.01$; NAcC: $(\mathrm{F}(2,31)=9.762, \mathrm{p}<0.01)$. Tukey post-hoc analysis revealed that in both the NAcSh and $\mathrm{NAcC}$ the classical conditioning group had a significantly lower c-Fos density compared to the operant and control groups $(\mathrm{p}<0.05)$.

\subsubsection{FosB}

A fixed factor ANOVA on the estimated density of FosB labeled cells in the NAcSh and NAcC (Figure 2.5) revealed a significant interaction between behavioral condition (control, operant and classical) and abstinence period in the NAcSh, $(\mathrm{F}(6,36)=$ $5.472, \mathrm{p}<0.001)$ but no interaction in the NAcC, $(\mathrm{F}(6,36)=1.860, \mathrm{p}>0.05)$. There was a main effect of behavioral condition in both regions (NAcSh: $(\mathrm{F}(2,36)=174.230, \mathrm{p}<$ 0.001); NAcC: $(\mathrm{F}(2,36)=112.919, \mathrm{p}<0.01)$ but no main effect of abstinence period in either region (NAcSh: $(F(3,36)=2.790, p>0.05)$; NAcC: $(F(3,36)=1.371, p>0.05)$. Tukey post-hoc analyses on behavioral condition revealed that the classically conditioned group had significantly fewer labeled neurons in the NAcSh and NAcC than the operant trained and control groups at all abstinence periods $(\mathrm{p}<0.01)$.

A One-way ANOVA on abstinence period within each behavioral condition revealed a main effect of abstinence period in the chocolate pellet treatment in the NAcSh, F $(3,16)=$ 5.267, $\mathrm{p}<0.01$. A one-way ANOVA on behavioral condition at each abstinence period revealed a main effect of treatment in the 24-hr. abstinence period in the NAcSh, F $(1,7)=$ $10.240, \mathrm{p}<0.05$. A Tukey post-hoc analysis revealed that the operant trained group had significantly more labeled neurons at 7 days than $24 \mathrm{hrs} .(\mathrm{p}<0.01)$ and that the control group showed significantly more labeled neurons at $24 \mathrm{hrs}$. compared to 28 days ( $\mathrm{p}<$ $0.05)$.

\subsubsection{Dendritic spine density}

A fixed factor ANOVA on MSN spine density in the NAcSh (Figure 2.6) revealed a main effect of abstinence period, $(\mathrm{F}(3,27)=3.135, \mathrm{p}<0.05)$ and a main effect of behavioral condition, $(\mathrm{F}(2,27)=39.333, \mathrm{p}<0.001)$ but no interaction, $(\mathrm{F}(6,27)=0.431, \mathrm{p}$ $>0.05)$. Tukey post hoc analysis on behavioral condition revealed that the classical and operant behavioral conditions had a significantly higher dendritic spine density compared to the control group $(\mathrm{p}<0.001)$.

\subsection{Discussion}

\subsubsection{Summary of main findings}

Incubation of craving for chocolate-flavored pellets and the impact of reinforcement contingency on craving was assessed using operant (contingent) and classical (non-contingent) conditioning behavioral designs. Following 10 days of either operant or classical conditioning using chocolate-flavored pellets as the reward, different 
groups of rats were tested following 24 hrs., 7 days, 14 days, or 28 days of forced abstinence. Behavioral measures during the test (correct lever presses, entries into the food hopper and locomotor activity) revealed similar behavioral output across all delays indicating the lack of an incubation of craving effect.

Ninety minutes following the test, brain tissue was processed and assessed for cFos and FosB labeling as well as dendritic spine density in the nucleus accumbens (NAc). c-Fos labeling was quantified to determine neuronal activation in the NAc following each abstinence test. The pattern of c-Fos labeling revealed no incubation effect at the neural level, mirroring the behavioral outcomes. FosB was quantified to determine long-term neuronal changes in the NAc. Groups that underwent classical conditioning showed fewer FosB-labeled neurons than both the operant-trained and the food restricted control groups at all abstinence periods.

Dendritic spine density in the NAcSh was assessed to determine changes in structural plasticity. Groups that underwent either operant or classical conditioning showed significantly more dendritic spines at all abstinence periods than food-restricted controls.

\subsubsection{Behavioral testing}

Incubation of craving has been reported in studies examining drugs of abuse and other rewarding stimuli whereby responding for cues associated with the rewarding substance increases over extended periods of abstinence. These studies result in similar incubation effects following self-administration despite differences in training time. A time-dependent increase in responding for cocaine-associated cues has consistently been shown following extended access to self-administered cocaine (Grimm et al., 2001, 2002; $\mathrm{Li}$ and Frantz, 2009). In a typical model of the incubation of cocaine craving, rats are trained to self-administer cocaine for two, $3-\mathrm{hr}$ sessions a day for 10 days with a 40 second time-out after each reward (Grimm et al., 2002). Following each reward presentation, a 5-second tone-light cue is presented and reward is limited to 40 per $3 \mathrm{hr}$. session (Grimm et al., 2002). The cue-induced reinstatement test consists of a 1-hr session where responses result in the presentation of a tone-light cue with a 40 second time-out (Grimm et al., 2002). A study by Li and colleagues (2009) used a similar behavioral design except training was $2 \mathrm{hrs}$. a day for 14 days instead of $6 \mathrm{hrs}$. a day for 10 days (Li and Frantz, 2009). Despite the differences in training, the incubation effect was similar (Grimm et al., 2001, 2002; Li and Frantz, 2009). When rats are trained to self-administer sucrose under similar conditions as cocaine, sucrose trained rats show increased responding for cues following 15 and 30 days of withdrawal compared to 24 hrs. (Grimm et al., 2002, 2005). Rats trained to self-administer a high fat pellet for 9 hrs./day for 14 days emit significantly more bar presses on day 21 compared to day 2 of withdrawal (Krasnova et al., 2014b). A key similarity between these reward selfadministration studies is the presentation of a tone-light cue paired with the reinforcer, a 
time-out period following the reward, and extinction testing with cues in the absence of reward.

In the present study, neither operant nor classical conditioning resulted in an incubation of craving effect. The choice of reward, behavioral design, and rodent (mouse versus rat) may be important factors in observing the incubation of craving phenomenon. Two notable discrepancies between our study and others is that 1) rats were trained for 1 hr. a day for 10 days and 2) we did not utilize an extinction without cues session. Following acquisition, self-administration studies often test for reward seeking by utilizing different testing procedures which can include extinction without cues, extinction with cues, or a combination of both, thus focusing on extinction and forced abstinence models (Venniro et al., 2016). A study by Nugent and colleagues (2017) investigated cue-induced reinstatement of cocaine or sucrose pellet seeking in C57BL/6J mice (Nugent et al., 2017). Self-administration was 2 hrs./day for 3 weeks (5 days/week) and an extinction session without cues test $(6 \mathrm{hr}$.) occurred prior to a responding for cues test. Following the extinction session, an incubation effect for cocaine, but not sucrose, was seen following 28 days of withdrawal (Nugent et al., 2017). Grimm and colleagues (2005) administered liquid sucrose for $6 \mathrm{~h} /$ day for 10 days and used testing procedures that began with extinction without cues (6 hr.) followed by responding for cues. An increase in responding for cues was observed following 30 days of forced abstinence compared to 1 day (Grimm et al., 2005). The main difference between these two sucrose studies was that one used liquid sucrose/rats (Grimm et al., 2005) and the other used sucrose pellets/mice (Nugent et al., 2017). In the absence of extinction without cues, incubation of palatable food (administered $9 \mathrm{hr}$./day for 14 days) and liquid sucrose (6 $\mathrm{hr}$./day for 10 days) craving has been demonstrated following 21 days of forced abstinence (Krasnova et al., 2014a; Uejima et al., 2007).

In addition to extinction and forced abstinence models, the incubation of craving has been studied by utilizing voluntary abstinence models (Caprioli et al., 2015, 2017; Venniro et al., 2017). These studies provide a choice between psychostimulant selfadministration and palatable food following periods of drug self-administration (Caprioli et al., 2017). Rats undergoing the voluntary abstinence procedure have been shown to abstain from drug self-administration when given a choice between drug and palatable food (Caprioli et al., 2017). Voluntary abstinence, Unlike forced abstinence, has been shown to prevent the development of incubation of heroin craving (Venniro et al., 2017). When the choice of palatable food is removed in rats that were trained to self-administer cocaine, cocaine seeking has been shown to increase (Quick et al., 2011). These studies provide evidence for the importance of incorporating palatable food into studies on the incubation of craving.

An additional variation in our study included the use of an FR2 schedule of reinforcement without the implementation of a time-out after reward delivery. Without the use of time-outs, it is possible rats became satiated before the end of each training period decreasing the reinforcing properties of the chocolate pellets. This is also the first incubation study that utilized chocolate-flavored pellets as the reward. Behavioral 
training with chocolate pellets clearly resulted in robust learning as evidenced by the increase in bar pressing over the training days and responding for the cues that continued at least 28 days following abstinence. In spite of this, there was no increase in behavioral responding over the different delay periods indicating the lack of an incubation effect. It would be worthwhile to determine if the lack of an incubation effect was due to the reward or behavioral training design by directly comparing other rewarding foods, such as sucrose or high fat pellets.

The use of non-contingent behavioral procedures in the study of incubation of craving have shown variable effects compared to contingent procedures. The conditioned place preference procedure (CPP) has been shown to elicit an incubation effect for cocaine and heroin ( $\mathrm{Li}$ et al., 2008; Lubbers et al., 2015). In the cases where an incubation effect was not found, the preference score remained similar across abstience delays for weeks following the last administration of cocaine or morphine suggesting that the drugs were rewarding (Mueller et al., 2002; Mueller and Stewart, 2000). In another non-contingent design, conditioned locomotor responding to cocaine-associated cues was assessed (Diehl et al., 2013a). Similar to the present study, cue-induced conditioned activity was observed following 3, 14, or 28 days of withdrawal without the presence of an incubation effect (Diehl et al., 2013a). The studies that did not result in incubation of craving are not unlike the present study, in that responding remains consistent across abstinence periods but an incubation effect is not evident.

In the present study, rats were food restricted to $90 \%$ of baseline weight throughout the study. In other studies involving food rewards, such as sucrose and high fat foods (Grimm et al., 2005; Krasnova et al., 2014a), food/water restriction was not used, or only used to initiate motivation for the reward. Food restriction may alter the rewarding effects of food and subsequent cue seeking behavior. For example, food deprivation in mice fed a high fat diet has been shown to enhance food seeking behaviors (Pérez-Ortiz et al., 2016). Food restriction has also been shown to enhance the selfadministration and locomotor effects of drugs of abuse (Cabeza de Vaca and Carr, 1998). In our study, it is possible that food restriction enhanced responding for cues at all abstinence periods and prevented the observation of an incubation effect.

Food seeking behavior may also be dependent on which phase of the light/dark cycle rats are tested in. Our studies were undertaken during the light phase of the day/night cycle rather than a reversed cycle. Testing for the incubation of craving has been undertaken in the light phase (Ma et al., 2014; Nugent et al., 2017) and dark phase of the light cycle (Grimm et al., 2003; Krasnova et al., 2014a) both of which have resulted in an incubation effect. While this could be a contributing factor (Roedel et al., 2006), it is likely not as important as the design of the behavioral training and testing conditions or choice of reward.

\subsubsection{C-Fos}

Both contingent and non-contingent administration of cocaine has been shown to increase c-Fos expression within the NAcSh, but only passive administration has been 
shown to increase c-Fos in the NAcC (Larson et al., 2010). In the incubation of craving, c-Fos levels were elevated in the NAcC and NAcSh following 30 days forced abstinence from sucrose compared to 1 day (Grimm et al., 2016). The increase in c-Fos coincided with an increase in bar pressing following 30 days of forced abstinence compared to 1 day (Grimm et al., 2016). In the present study, c-Fos labeling remained relatively stable across all abstinence periods and was associated with the lack of behavioral incubation. This provides additional evidence that neither contingent nor non-contingent chocolate pellet delivery induces a robust incubation of craving effect.

Food-restricted controls displayed similar c-Fos labeling compared to the operant trained group. Overall, the classically conditioned group displayed lower c-Fos levels than the operant and control groups. The testing for classical conditioning involved the addition of the levers to the chambers, providing a new variable that may not be immediately associated with the reward-related cues resulting in less activation in the NAc. Cues previously associated with rewarding foods, which have acquired incentive value, have been shown to induce c-fos mRNA and c-Fos protein expression within the NAc (Figlewicz et al., 2011; Flagel et al., 2011). Besides the incentive value of foodpaired cues, anticipation of scheduled food has been shown to induce c-Fos expression in the NAc (Blancas et al., 2014). Food anticipation may explain why the control group had similar c-Fos expression as the operant group. Restricted feeding schedules to normal chow has been shown to result in elevated c-Fos expression within the NAc (Mendoza et al., 2005). All animals, irrespective of group, were fed regular chow at approximately the same time every day. In behaviorally-trained groups, testing was performed approximately 1 hour prior to normal feeding times and took place at similar times of day that prior training took place.

\subsubsection{FosB}

DeltaFosB accumulates in the NAc following administration of addictive drugs and natural rewards (Hadad and Knackstedt, 2014; Kelz et al., 1999; Larson et al., 2010; Nestler, 2004, 2008; Pitchers et al., 2013; Sharma et al., 2013). The availability of extracellular DA and subsequent D1 binding may play a role in the expression of DeltaFosB within the NAc. Food restriction alone has been shown to increase DA in the NAc (Pothos et al., 1995) and increase DA receptor signaling (Carr et al., 2003). Blockade of D1 receptors in the presence of elevated DA in the NAc has been shown to attenuate DeltaFosB induction following morphine administration (Muller and Unterwald, 2005). Stamp and colleagues (2008) explored the effect of food restriction on DeltaFosB expression in the NAc of rats (Stamp et al., 2008). Food restriction significantly increased DeltaFosB expression compared to free-fed controls (Stamp et al., 2008).

The response of DA release differs depending on whether a natural reward is administered in a contingent or non-contingent manner whereas DA release in response to drugs of abuse does not (Chiara and Di Chiara, 2002). A study by Bassareo and 
colleagues (2015) explored the effect of response contingency on NAcSh and NAcC extracellular DA following sucrose delivery (Bassareo et al., 2015b). In rats that were repeatedly fed sucrose pellets in a non-contingent manner, there was an increase in extracellular DA on the first trial that habituated on the second and third trial. In rats that underwent operant conditioning (contingent administration), extracellular DA release did not habituate over trials (Bassareo et al., 2015b).

In the present study, food restricted controls displayed similar FosB expression as rats that underwent operant conditioning. This is not surprising, as food restriction and operant conditioning have been shown to increase extracellular DA. In the classically conditioned animals, the lower expression of FosB may be the result of DA habituation within the NAc in response to non-contingent administration of the chocolate flavored pellets. This could decrease DA binding to DA receptors thus resulting in less FosB expression compared to the other groups, which may have had higher extracellular DA available for binding.

\subsubsection{Dendritic Spines}

Chronic contingent and non-contingent administration of drugs, including amphetamine and cocaine, is associated with increased spine density on MSNs in the NAcSh (Robinson and Kolb, 2004). Self-administration of food reward has also been shown to increase dendritic spine density within the NAcSh (Guegan et al., 2013; Mancino et al., 2016). In incubation of craving models, dendritic spine density has been shown to increase in the NAc following 36 days of withdrawal from cocaine and following 30 days of forced abstinence from normal chow (Christian et al., 2016; Dingess et al., 2017b). These long-term changes are correlated with a variety of motivated behaviors, such as behavioral indices of craving, which may lead to relapse (Robinson and Kolb, 2004). In the present study, dendritic spine density was consistently higher at all abstinence periods in both the classical and operant groups than food restricted controls. The elevated spine densities coincide with food seeking behavior during the testing periods and may reflect long-term changes at the neural level indicative of the incentive value of reward-related cues. While spine densities were higher in both trained groups than the food-restricted controls, there were no significant differences in spine density between the operant and classical groups. These results suggest that changes in dendritic spine density may be attributed to the learning that takes place during contingent or non-contingent reinforcer delivery.

There is strong evidence that LTP associated with craving involves the insertion of new calcium permeable (CP)-AMPA receptors into the post-synaptic membrane (Malinow and Malenka, 2002). Preventing the influx of $\mathrm{Ca} 2+$ into neurons has been shown to prevent dendritic spine changes highlighting the importance of $\mathrm{Ca} 2+$ in structural plasticity (Yasumatsu et al., 2008). The amount of time necessary for CPAMPA receptor accumulation has been shown to vary depending on the type of drug/food being administered and withdrawal time from the substance. Ingestion of 
"junk-food" has been shown to induce long lasting increases in CP-AMPA receptors, especially in rats susceptible to obesity (Oginsky et al., 2016). This accumulation of CPAMPA receptors was present following 1,14 , and 21 days of junk-food deprivation, suggesting that upregulation is rapid and persistent (Oginsky et al., 2016). Following withdrawal from self-administered cocaine, CP-AMPA receptors remain similar to saline controls during the first 3 weeks followed by an increase in CP-AMPA receptors around day 30 coinciding with increased responding for cues (Wolf and Tseng, 2012). In contrast, CP-AMPA receptor accumulation following self-administration of methamphetamine is at its highest approximately 1 week after withdrawal even though seeking behavior was similar following both 7 and 30 days (Scheyer et al., 2016). In the present study, it is plausible that the expression of CP-AMPA receptors was increased in response to chocolate flavored pellets or behavioral training. The insertion of new CPAMPA receptors could have facilitated the increase in dendritic spine density via elevated $\mathrm{Ca} 2+$ influx into the cell.

\subsection{Conclusions}

Neither contingent nor non-contingent administration of chocolate flavored pellets resulted in an incubation of craving effect but resulted in robust responding across all abstinence periods. Administration of chocolate flavored pellets was associated with an increase in dendritic spine density in the NAcSh suggesting ingestion of chocolate flavored pellets synaptically alters the NAcSh for at least 28 days following forced abstinence. Differences between contingent and non-contingent administration was also evident in immediate early gene labeling. This suggests that the method of administration has differential effects on the NAc, but the functional significance of this has yet to be elucidated.

\section{Conflict of Interest}

The authors declare no conflict of interest, financial or otherwise.

\section{Author Contributions}

Conceived and designed the experiments: ENT MH. Performed the experiments: ENT SL. Analyzed the data: ENT. Writing of the manuscript: ENT MH.

\section{Funding}

This research was supported by a National Science and Engineering Research Council of Canada Discovery grant to MH (\#341673). 
(A) Tyrosine Hydroxylase

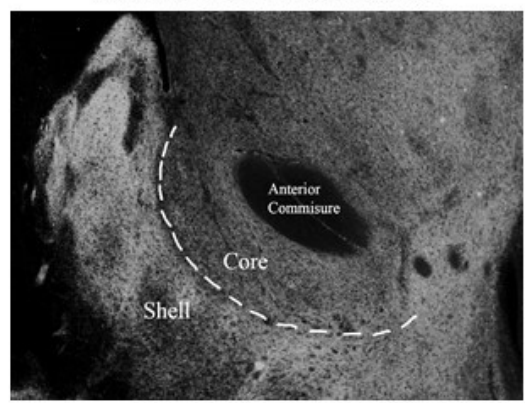

(B) C-Fos

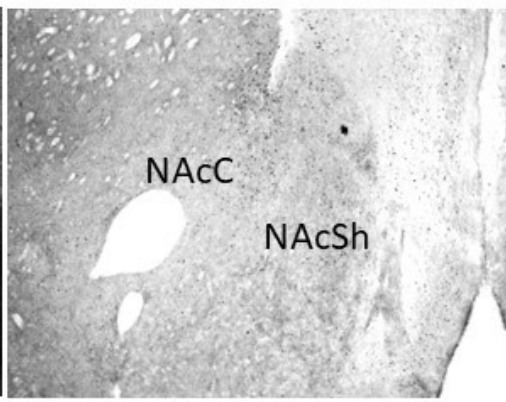

6.

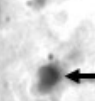

-

$\checkmark$

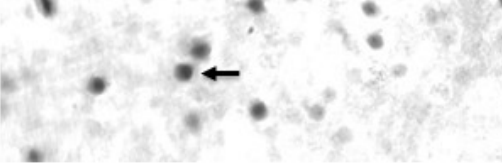

(C) FosB
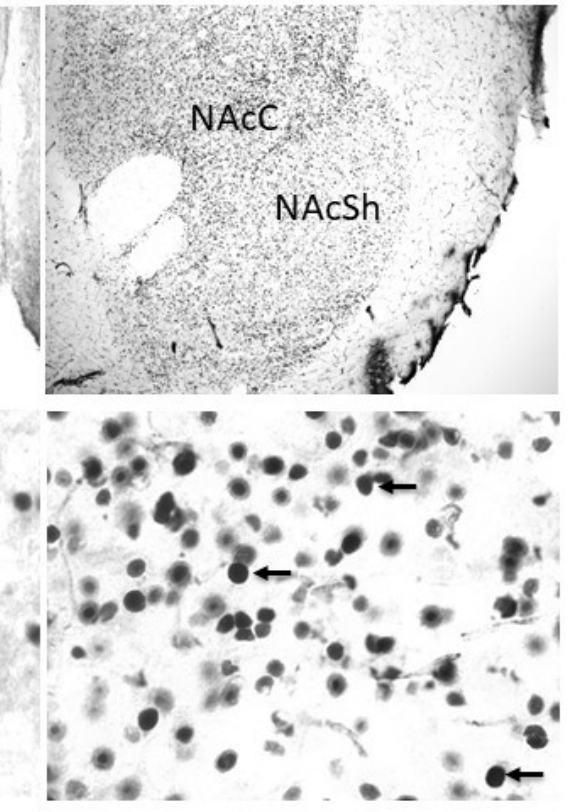

Figure 2.1. Representative images of NAcC and NAcSh delineation and labeling. (A) Tyrosine hydroxylase stained image at $4 \mathrm{x}$ to show the location of the NAcC and NAcSh. (B) Representative images of c-Fos labeling in the NAcC and NAcSh at 4x and 60x. (C) Representative images of FosB labeling in the NAcC and NAcSh at 4x and 60x. 
Day 10 Acquisition

(A)

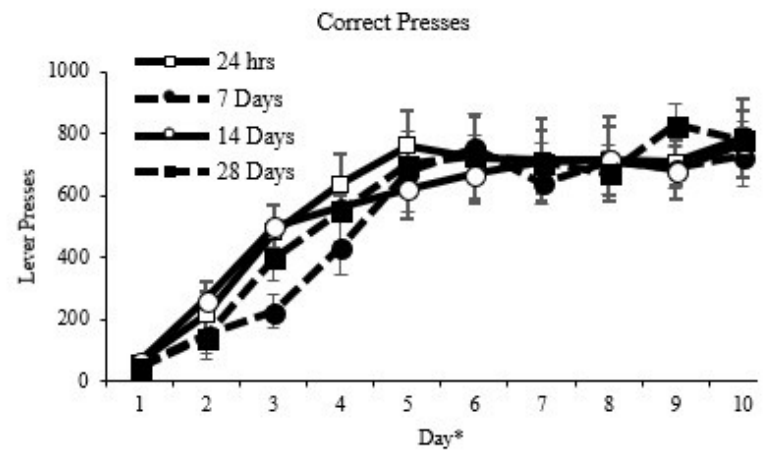

(B)

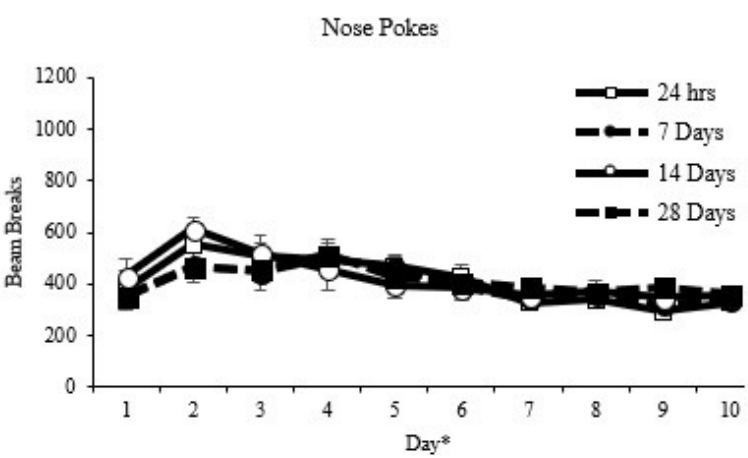

(C)

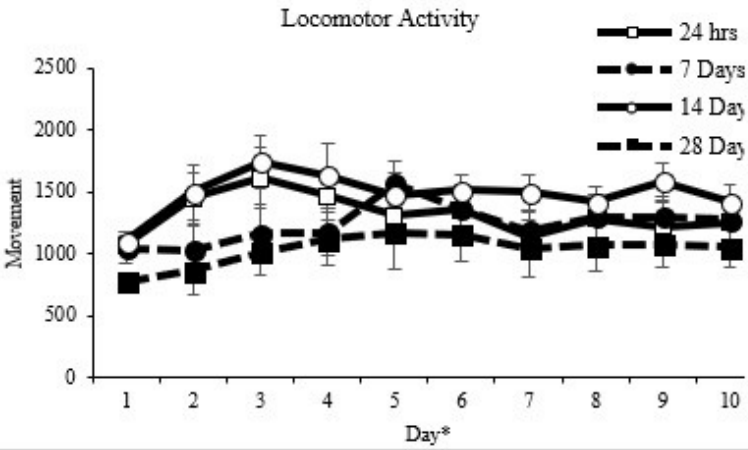

Testing

(D)

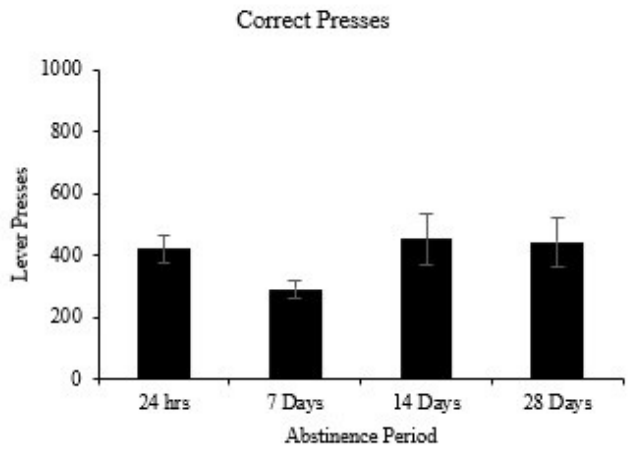

(E)

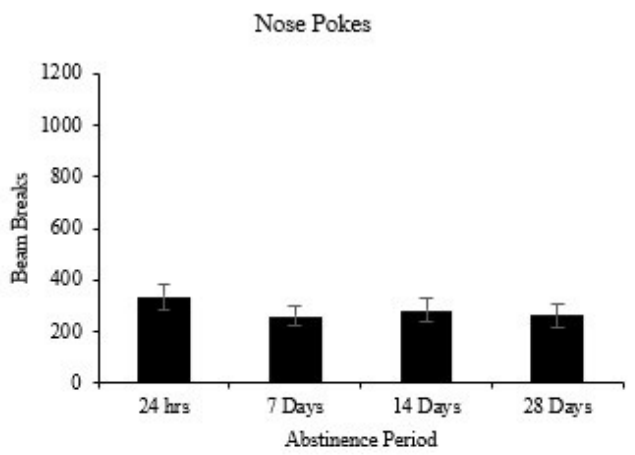

(F)

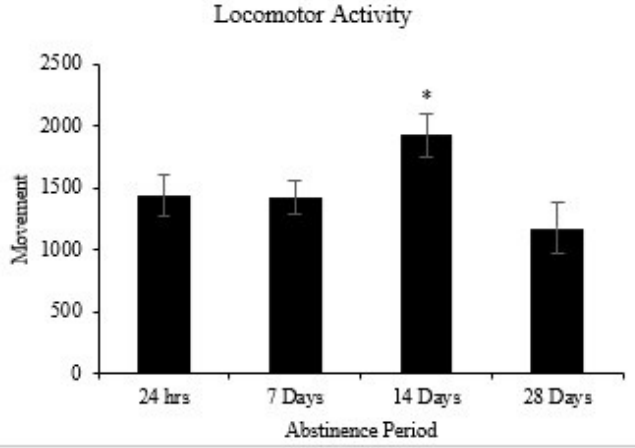

Figure 2.2. Operant conditioning (mean \pm SEM). (A-C) Total correct lever presses, nose pokes, and locomotor activity are shown for each of the 10 days of acquisition. ${ }^{*} p<0.01$ significant effect of day (DF) Total correct lever presses, nose pokes, and locomotor activity are shown for the 60 -minute test. * p $<$ 0.05 compared to the 28-day abstinence period. 
Day 10 Acquisition

(A)

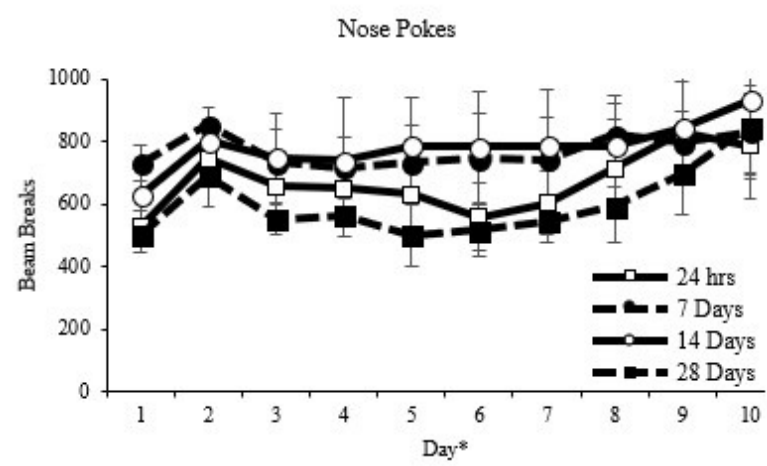

(B)

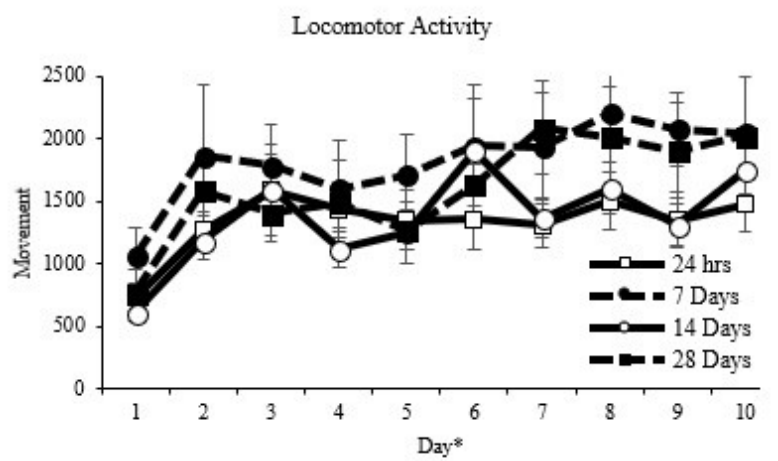

Testing

(C)

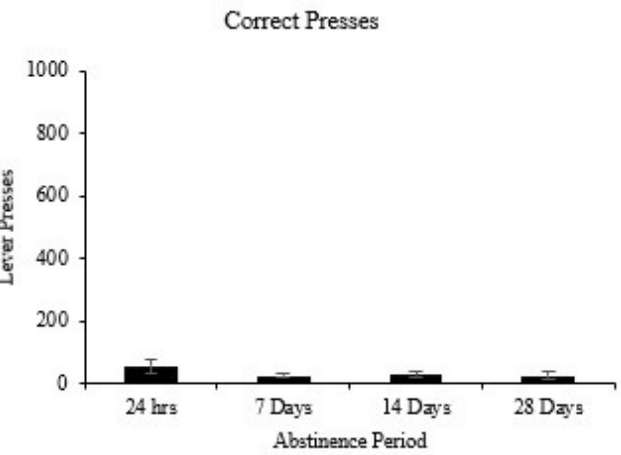

(D)

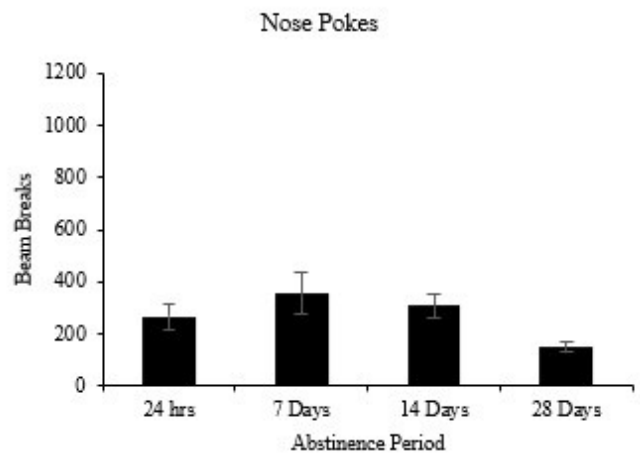

(E)

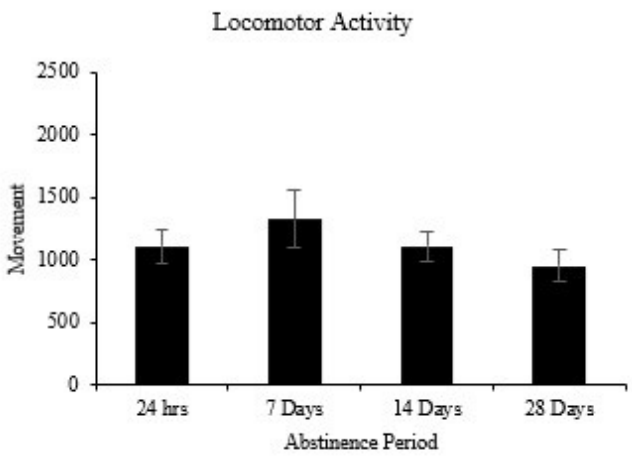

Figure 2.3. Classical conditioning (mean \pm SEM). (A-B) Total nose pokes and locomotor activity are shown for each of the 10 days of conditioning. $* \mathrm{p}<0.01$ significant effect of day (C-E) Total correct lever presses, nose pokes, and locomotor activity are shown for the 60 -minute test. 
(A)

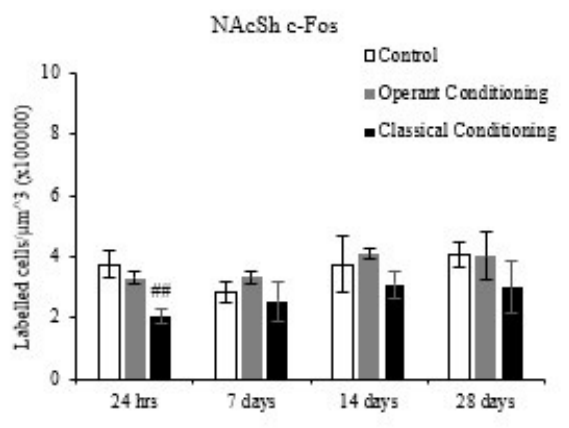

(B)

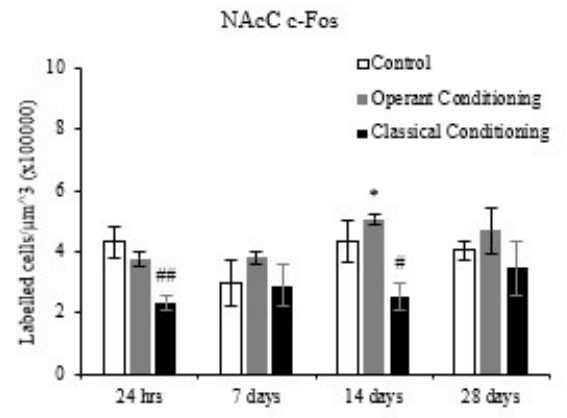

(C)

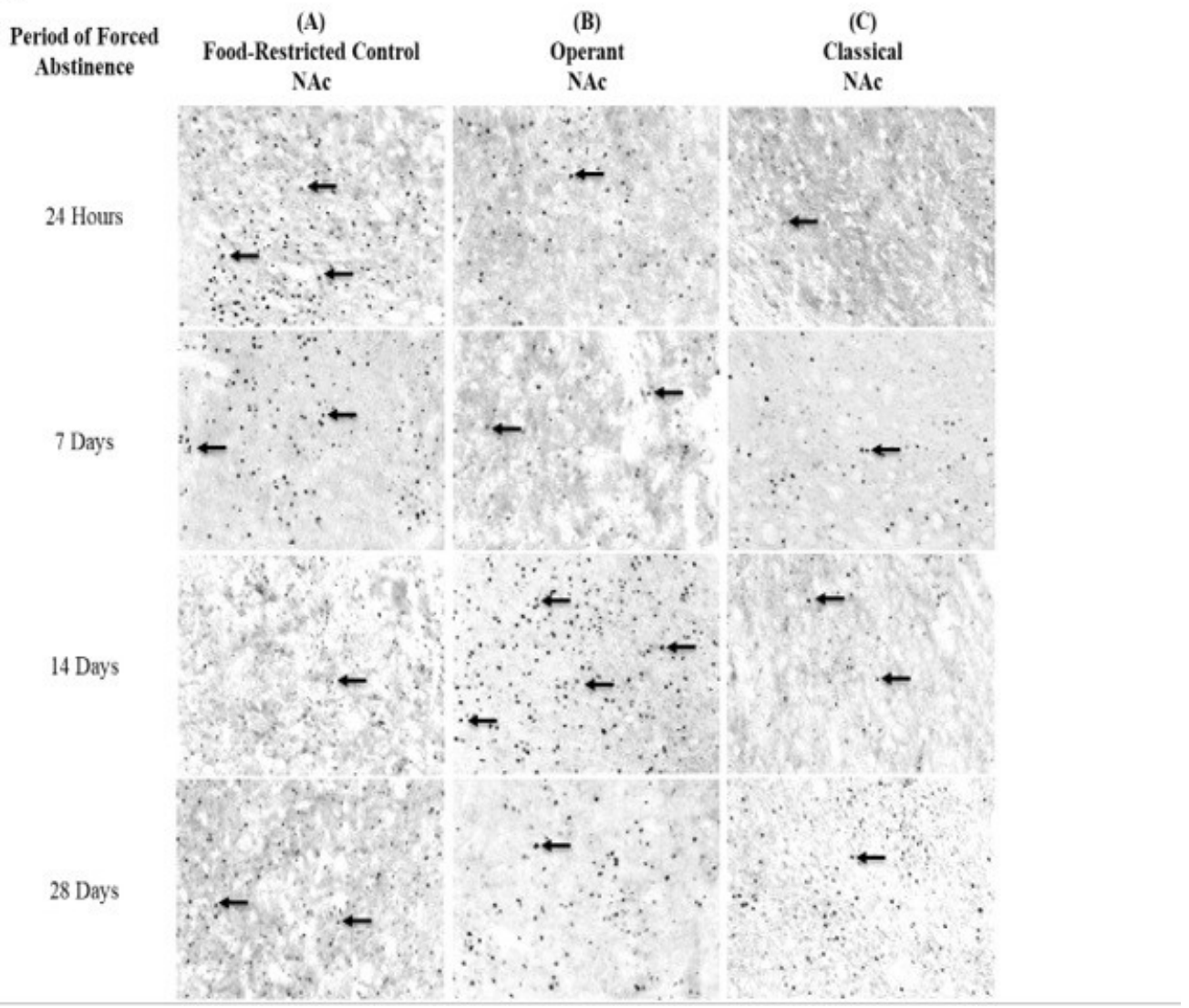

Figure 2.4. Average cell density (mean cell count/volume $\left[\mathrm{mm}^{3}\right]$ ) of c-Fos labeled neurons (mean $\pm \mathrm{SEM}$ ) (A) NAcSh labeled neurons in rats that underwent operant and classical conditioning compared to food restricted controls. ${ }^{\#} \mathrm{p}<0.01$ compared to operant conditioning and controls at $24 \mathrm{hrs}$. (B) NAcC labeled neurons in rats that underwent operant conditioning compared to food-restricted controls. * $\mathrm{p}<0.05$ compared to operant conditioning at $24 \mathrm{Hrs}$. ${ }^{\#} \mathrm{p}<0.01$ compared to operant conditioning and controls at $24 \mathrm{hrs} .{ }^{\#} \mathrm{p}<0.05$ compared to operant conditioning at 14 days. (C) Representative images of c-Fos labeling in the NAc in rats that underwent operant and classical conditioning and food restricted controls $20 x$. 
(A)

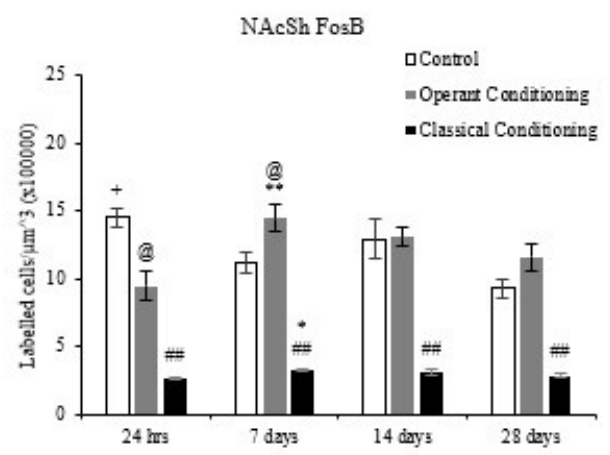

(B)

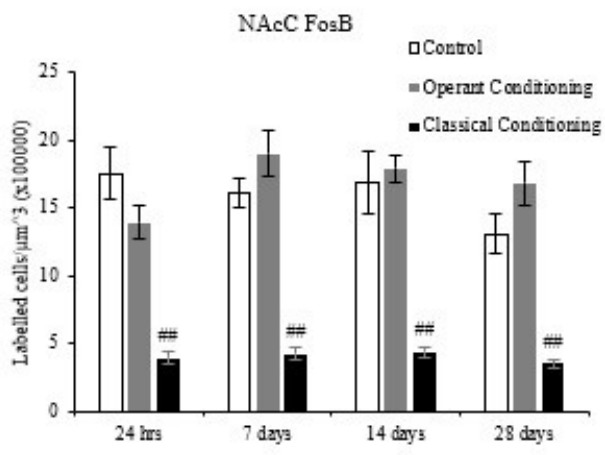

(C)

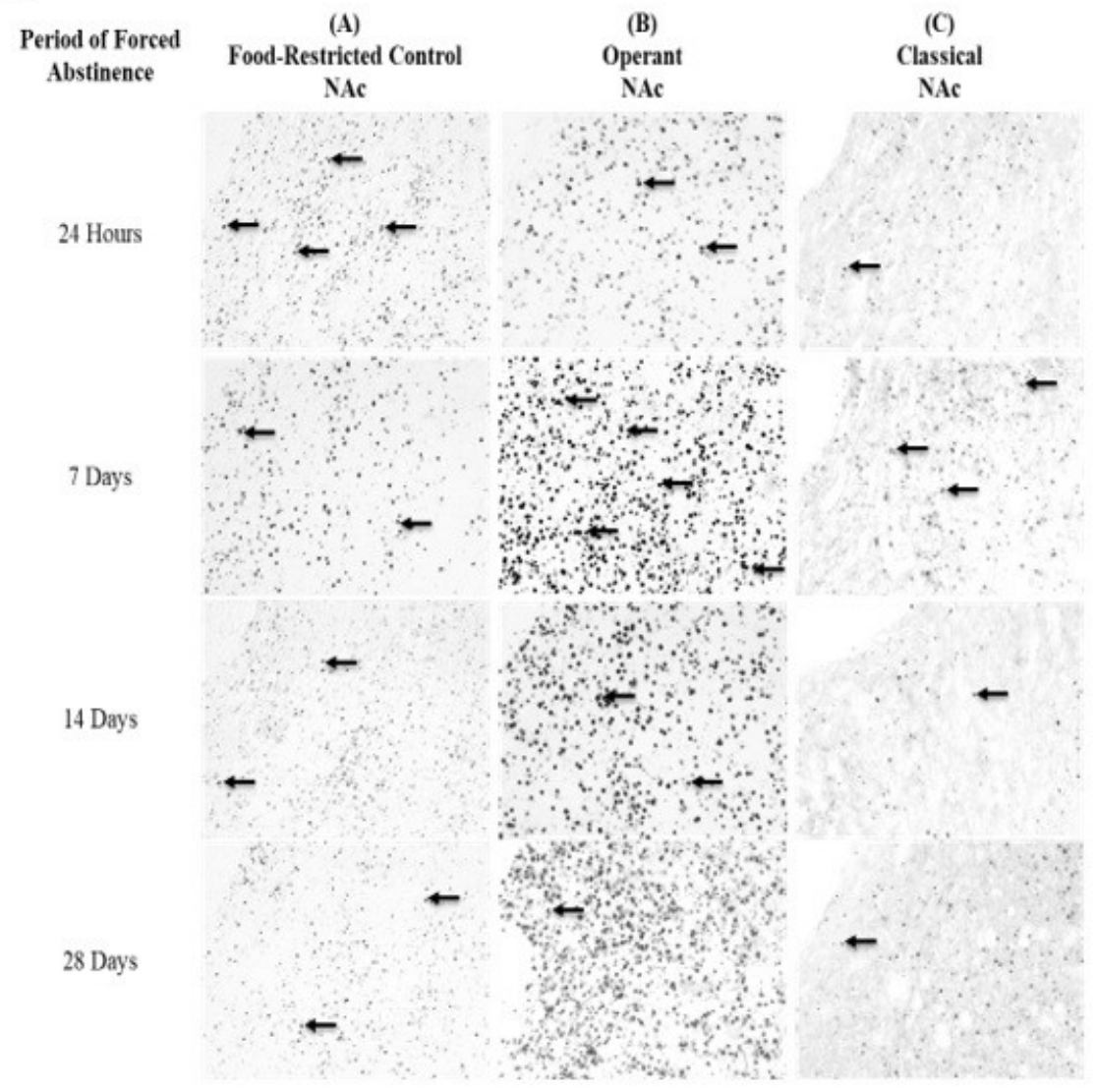

Figure 2.5. Average cell density (mean cell count/volume $\left[\mathrm{mm}^{3}\right]$ ) of FosB labeled neurons (mean $\pm \mathrm{SEM}$ ). (A) NAcSh labeled neurons in rats that underwent operant and classical conditioning compared to foodrestricted controls. ${ }^{\#} \mathrm{p}<0.01$ compared to operant conditioning and control at the same abstinence period. (a) $\mathrm{p}<0.05$ compared to control at the same abstinence period. $* \mathrm{p}<0.05$ compared to classical conditioning at $24 \mathrm{hrs}$. $* * \mathrm{p}<0.01$ compared to operant conditioning at $24 \mathrm{hrs}$. ${ }^{+} \mathrm{p}<0.05$ compared to control at 28 days. (B) NAcC labeled neurons in rats that underwent operant and classical conditioning compared to food-restricted controls. ${ }^{\#} \mathrm{p}<0.01$ compared to operant and classical conditioning at the same abstinence period. (C) Representative images of FosB labeling in the NAc in rats that underwent operant and classical conditioning and food restricted controls 20x. 
(A)

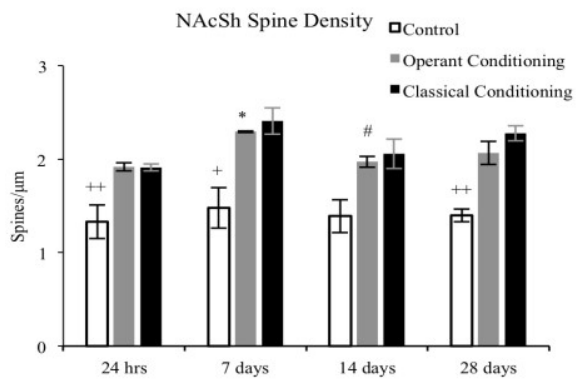

(B)

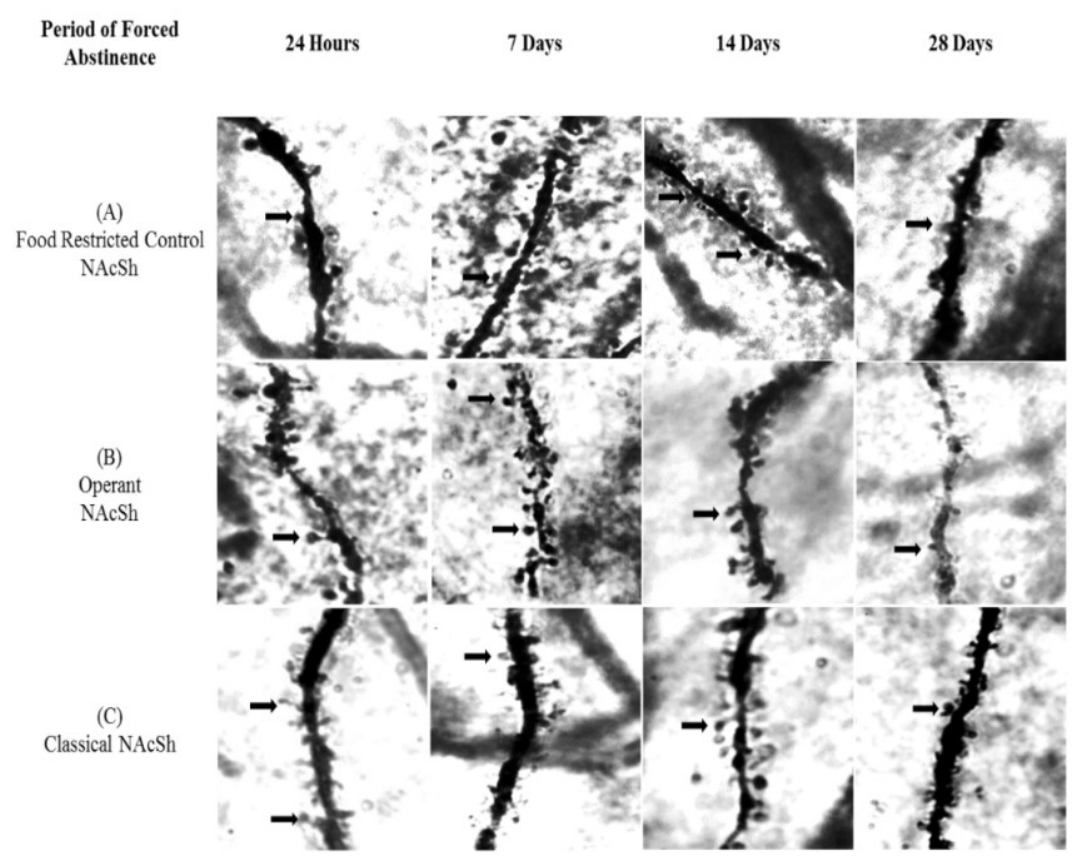

Figure 2.6. (A) Average dendritic spine density (spines/ $\mu \mathrm{m}$ ) on MSNs in the NAcSh in rats that underwent operant and classical conditioning compared to food-restricted controls (mean $\pm \mathrm{SEM}$ ). ${ }^{+} \mathrm{p}<0.05$ compared to operant and classical conditioning at 7 days. ${ }^{++} \mathrm{p}<0.01$ compared to operant and classical conditioning at same abstinence period. ${ }^{*} \mathrm{p}<0.05$ compared to operant conditioning at 24 hrs. ${ }^{*} \mathrm{p}<0.05$ compared to control at 14 days. (B) Representative images of dendritic spines on MSNs in the NAcSh in rats that underwent operant and classical conditioning for chocolate pellets and food restricted controls 100x. 


\section{Chapter 3}

\section{Exploring Time-Dependent Changes in Conditioned Place Preference for Food Reward and Associated Changes in the Nucleus accumbens}

Erin W. Noye Tuplin and Matthew R. Holahan

\subsection{Abstract}

Rationale: Incubation of craving is defined as a time-dependent increase in responding for cues associated with reward. The conditioned place preference (CPP) procedure has been used to study this phenomena with rewarding drugs such as cocaine and methamphetamine. There have been fewer studies that use the CPP design to study food rewards and the incubation of craving. Because chocolate is often a highly rewarding and craved food type, we used the CPP design to examine whether chocolate would induce an incubation of craving response.

Objective: The present study examined whether rats trained in a CPP behavioral design would display an incubation of craving response for chocolate flavored pellets or milk chocolate chips at the behavioral and neural levels following periods of forced abstinence from chocolate.

Methods: Rats were conditioned for 10-days using an unbiased CPP design. Groups then underwent abstinence from the chocolate for $24 \mathrm{hrs}, 7$ days, 14 days, or 28 days at which point they were tested for CPP. Following the test, brains underwent immunohistochemical staining for c-Fos and DeltaFosB as well as Golgi staining to assess dendritic spine density in the nucleus accumbens (NAc).

Results: Neither chocolate pellet nor milk chocolate chip reward resulted in an incubation of craving response. Only the chocolate pellet group displayed a CPP response, which diminished by the 14-day abstinence period. Overall, c-Fos and DeltaFosB labelling were significantly higher for the chocolate pellet group compared to the control group at the 7-day abstinence period. At the 28-day abstinence period, the chocolate pellet group had significantly more total spines than the control and milk chocolate groups.

Conclusions: The presented behavioral model was not conducive in modeling incubation of food craving on a neural or behavioral level. We have shown that food 
type may be important in the development of CPP and associated neural changes. Chocolate pellets are rewarding but, as evidenced by limited neural changes, these food rewards may not be sufficient to induce enhanced craving following long periods of forced abstinence.

\subsection{Introduction}

In animal models of craving, substance-seeking behavior can be defined as responding for substance-related cues in the absence of the substance (Grimm et al., 2001, 2011a; Lu et al., 2004). Time-dependent enhancement in reward seeking behavior following abstinence is termed "incubation of craving" (Grimm et al., 2001, 2002; Lu et al., 2004). Following a period of abstinence from a rewarding substance, rats develop increased cue-induced responding on day 30 compared to day 1 of abstinence (Calu et al., 2007; Grimm et al., 2001, 2002; Halbout et al., 2014; Lu et al., 2004). Incubation of craving has been noted for cocaine (Calu et al., 2007; Grimm et al., 2001, 2002; Halbout et al., 2014; Lu et al., 2004), methamphetamine (Li et al., 2015b, 2015a; Shepard et al., 2004), nicotine (Abdolahi et al., 2010; Funk et al., 2016), heroin (Shalev et al., 2001), alcohol (Bienkowski et al., 2004), sucrose (Grimm et al., 2005; Lu et al., 2007), and other rewarding foods (Darling et al., 2016; Krasnova et al., 2014d) using self-administration experimental designs.

The study of craving with non-contingent experimental designs are based on classical conditioning principles and include the conditioned place preference (CPP) method (Bardo and Bevins, 2000; Tzschentke, 2007). The CPP method capitalizes on the ability of rats to associate specific environmental cues with a rewarding substance such as morphine (Sun et al., 2017), opiates (Li et al., 2008), or cocaine (Lubbers et al., 2015). In incubation of craving studies using the CPP methodology, drug seeking behavior elicited by the conditioned cues may be quantified by different measures (Duan et al., 2016; Sun et al., 2017). For example, the preference ratio may be representative of a memory for the rewarding substance whereas compartment entries may more closely reflect reward-seeking behavior (Sun et al., 2017).

The nucleus accumbens (NAc) is a focus in the study of the neural substrates that subserve compulsive behaviors related to obtaining reward (Adinoff, 2004; Chaudhri et al., 2010; Di Chiara, 2002; Grimm et al., 2011b; Nestler, 2012; Wolf and Tseng, 2012). Rewarding substances, including natural rewards and drugs of abuse, have been shown to alter activity in the nucleus accumbens (NAc) (Breiter et al., 1997; Small et al., 2003; Wise, 2006). Regional differences between the NAcSh and NAcC have been noted in immediate early gene expression following both chronic and acute substance administration (Larson et al., 2010; Renthal et al., 2008). Self-administered cocaine is associated with an increase in c-Fos labelling in the NAcSh but not the NAcC and acute cocaine has less of an effect on NAcSh c-Fos expression (Larson et al., 2010). Chronic cocaine administration has been associated with an increase in DeltaFosB in both the NAcSh and NAcC (Larson et al., 2010). Following abstinence from cocaine, but without a cue reinstatement test, DeltaFosB is significantly reduced by week 3, FosB by 24 hrs, and c-Fos by $24 \mathrm{hrs}$ in the NAcSh and NAcC (Larson et al., 2010). Following a cue- 
reinstatement test for sucrose-related cues, c-Fos labeling is elevated after 30 days compared to 1 day in both the NAcSh and NAcC (Grimm et al., 2016).

Changes in neuronal structure, such as alterations in dendritic spines and spine morphology, have been associated with administration of rewarding substances (Guegan et al., 2013; Kobrin et al., 2016; Nyberg, 2014; Quintero, 2013). Administration of cocaine or amphetamine is associated with an increase in total spine density on medium spiny neurons (MSNs) in the NAcSh and NAcC (Robinson and Kolb, 1999, 2004). The increase in dendritic spine density associated with cocaine or amphetamine has been shown to persist for at least one month after drug abstinence (Robinson and Kolb, 2004). Spine morphology has been shown to change temporally and depend on chronicity of substance administration and abstinence time. Mushroom type spines appear to be more stable and can persist for months whereas thin spines tend to change more rapidly, suggesting mushroom spines may be representative of longer-term plasticity (Bourne and Harris, 2007; Huber and Menzel, 2004). Cocaine treatment results in larger spines, suggesting synaptic strengthening, whereas abstinence results in an increased density of smaller spines (Robinson and Kolb, 1999; Spiga et al., 2014). Following 30 days of abstinence from chow, an increase in mushroom type spines has been observed in the NAcSh and NAcC (Dingess et al., 2017a).

The macronutrient composition of food has been shown to affect patterns of behavioral sensitization (Mason, 2009; Serafine et al., 2014), immediate early gene induction (Chen et al., 2011; Dela Cruz et al., 2015), and neuron structure (Dingess et al., 2017a). The consumption of high fat chow, but not intake of sucrose or saccharin, facilitates the development of locomotor sensitization to cocaine (Serafine et al., 2014). In the NAcC, c-Fos expression is elevated following glucose and corn oil ingestion but not following saccharin or fructose (Dela Cruz et al., 2015). Incubation of craving appears to be independent of macronutrient composition (Darling et al., 2016), but the magnitude of the incubation effect may depend on the macronutrient composition (Dingess et al., 2017a). Both chow and high fat foods result in increased responding for cues at 30 days compared to 1 day of forced abstinence, but high fat chow resulted in significantly more responding (Dingess et al., 2017a). In this instance, the chow group displayed significantly more thin dendritic spines in the NAcSh and NAcC at 1 day of forced abstinence compared to the naïve control and high-fat groups (Dingess et al., 2017a). Mushroom spine density was higher in the NAcSh in the chow group following 30 days of forced abstinence compared to control and high fat whereas in the NAcC, mushroom spine density was higher for both the high fat and chow groups compared to the control group (Dingess et al., 2017a).

There are currently a dearth of studies exploring incubation of craving for rewarding foods utilizing non-contingent designs, such as conditioned place preference (CPP). In human populations, chocolate is considered one of the most craved foods in North America (Nasser et al., 2011). We have previously studied incubation of craving for chocolate flavored pellets in a self-administration model, where rats acquire robust responding for pellets by day 5 of acquisition (Davis-MacNevin et al., 2013; Noye Tuplin et al., 2018; Tuplin et al., 2015). Chocolate flavored pellets differ in macronutrient composition and lack vital chocolate components (cocoa) compared to chocolate consumed by the human population. To determine if the rewarding effects of chocolate 
flavored pellets are similar to real chocolate, the chocolate pellet group was compared to a milk chocolate food group. We have previously shown that rats acquire food-seeking behavior in response to discrete environmental cues previously paired with noncontingent chocolate pellet delivery (Noye Tuplin et al., 2018) but the previous model would not allow for the dispensing of milk chocolate chips. The present study utilized an unbiased, non-contingent, CPP procedure to explore the incubation of craving for chocolate flavored pellets or milk chocolate chips and to determine any differences between the two foods in the ability to produce time-dependent changes in CPP.

The CPP followed an unbiased procedure where half of the rats received the reward in one compartment and the other rats in the adjacent compartment. A control group served two purposes: to ensure the unbiased nature of the apparatus and to serve as a control for immunohistochemical and dendritic spine analysis. Rats were tested for their side preference following $24 \mathrm{hrs}, 7$ days, 14 days, or 28 days of forced abstinence from either chocolate flavored pellets or milk chocolate chips. The labeling of c-Fos and FosB was quantified within the NAcSh and NAcC to determine short and long-term changes in functional aspects of these neurons. FosB is a common marker of neuron activity in addiction studies and accumulates over time following repeated exposure to a rewarding substance (Nestler, 2004, 2008, 2015). The c-Fos protein represents an index of neuronal activity during the CPP testing procedure. Spine density and spine morphology (thin, stubby, filopodia, mushroom) were measured on medium spiny neurons (MSNs) within the NAcSh and NAcC to determine any differences between the food rewards and across abstinence periods.

\subsection{Methods}

\subsubsection{Subjects}

A total of 96 male Long Evans rats (250-275 grams) were obtained from Charles River, Quebec. Rats were housed individually in clear plastic cages $(25 \times 20 \times 45 \mathrm{~cm})$, given water ad libitum under a 12-hour light/dark cycle (lights on at 8:00 am), and tested during the light phase. Food was restricted to $90 \%$ baseline, which was maintained until the end of the study. Prior to behavioral testing, rats were handled for 5 minutes daily. Principles of laboratory animal care were followed, and all procedures were conducted in accordance with the Canadian Council on Animal Care. The Carleton University Animal Care Committee approved the protocol.

\subsubsection{Conditioned place preference procedure}

All behavioral training and testing was performed using four identical CPP apparatuses. An apparatus consisted of three chambers, two large chambers separated by a small third chamber. One chamber was white with a smooth floor and the other chamber was black with a gridded floor, the middle walk-way chamber was gray with a smooth floor. Removable gray doors separated the middle walkway from the two large chambers. Prior to training, rats were assigned to one of 12 groups $(n=8)$ which were matched according to weight so that each group had a similar average starting weight. Rats were assigned to an abstinence period of either 24 hrs., 7 days, 14 days, or 28 days 
and further divided into three food groups within each abstinence period: control (no food), chocolate flavored pellets, and milk chocolate chips (Table 1).

\begin{tabular}{|l|l|l|}
\hline$\%$ & Chocolate Flavored Pellets & Milk Chocolate Chips \\
\hline Protein & 18.4 & 6.7 \\
\hline Fat & 5.5 & 26.7 \\
\hline Carbohydrate & 59.1 & 66.7 \\
\hline
\end{tabular}

Table 3.1 Percent of protein, fat, and carbohydrates in chocolate flavored pellets and milk chocolate chips.

An unbiased CPP design was utilized for the experiment and the unbiased nature of the apparatus was confirmed with the control group. Each group was counterbalanced so that half of the rats received food reward on the white smooth side and half the rats received the food reward on the black, gridded side. The opposite chamber (non-reward; unpaired) remained empty to prevent a preference for the unpaired side. Under food restricted conditions, animals may develop a preference for regular chow, suggesting that normally less rewarding food can induce seeking behaviors (Zombeck et al., 2008). A control group underwent the same experimental procedure but never received food on either side.

Prior to conditioning, a habituation day was conducted when rats could move freely throughout the entire apparatus for 20 minutes. Following the habituation day, rats underwent 10 days of conditioning for 20 minutes per day. Approximately 90-120 minutes following each conditioning day, rats were weighed and received the appropriate amount of food to allow for maintenance of weight at $90 \%$ baseline. Restricted feeding schedules were also maintained throughout the abstinence periods. On odd numbered days $(1,3,5,7,9)$, rats were placed on the unpaired side and did not receive any food. On even days $(2,4,6,8,10)$, rats were placed on the side paired with food and given $4 \mathrm{~g}$ of either chocolate-flavored pellets or milk chocolate chips; control rats did not receive food while in the apparatus. During testing, rats were placed in the middle compartment and had access to the entire apparatus for 20 minutes. Testing was video-recorded, and time spent in each compartment and entries into each compartment were calculated for each 10-minute interval. A preference ratio was calculated using the following formula: (paired/(paired+unpaired)) x 100. A ratio above 50\% represented a preference for the paired compartment.

\subsubsection{Immunohistochemistry}

Ninety minutes following testing, rats were given an overdose of sodium pentobarbital and perfused with saline. One hemisphere, alternating between left and right, was immersion fixed in $4 \%$ paraformaldehyde/0.01M phosphate buffer solution $(\mathrm{pH}$ 7.4) and the other hemisphere was placed in a Golgi Fix solution protected from light. Following $24 \mathrm{hrs}$, the paraformaldehyde-immersed tissue was cryopreserved via immersion in a $30 \%$ sucrose $/ 0.1 \mathrm{M}$ phosphate buffer solution for a minimum of 4 days. The tissue was then sectioned on a cryostat at $60 \mu \mathrm{m}$ and placed in a $0.1 \%$ sodium azide/0.1 M phosphate buffer solution ( $\mathrm{pH} 7.4$ ). 
FosB and c-Fos immunohistochemistry followed similar staining procedures as previously reported (Noye Tuplin, et al., 2018). Two sections with the NAc from each rat were chosen for FosB and two other sections were chosen for c-Fos immunohistochemical staining (bregma 2.7-3.2). Sections were incubated overnight at room temperature in the primary antibody (1:5000 anti-FosB host: mouse Abcam cat \# ab1 1959 or 1:5000 anti-c-Fos host: rabbit Synaptic Systems cat \# 226003). The following day, tissue underwent three 10-minute washes in PBS-TX followed by incubation in the secondary antibody (FosB - 1:1000 goat anti-mouse IgG: Vector; c-Fos $-1: 500$ goat anti-rabbit IgG: Vector) for $1 \mathrm{hr}$ at room temperature. Following development, the tissue was washed twice in PBS for 5 minutes then mounted onto slides, dehydrated, and coverslipped.

\section{Immunohistochemistry quantification}

A counting method based on unbiased stereology principles using the optical fractionator method was used to estimate the number of FosB and c-Fos labelled cells in the NAcC and NAcSh subregions. The NAc was traced at $2 \mathrm{x}$ magnification using an Olympus BX51 bright field microscope with a motorized stage (Olympus Canada, Markham, ON) and FosB and c-Fos labelled cells were quantified using a 60x magnification lens (oil immersion, NA 135) (see Figure 3.1A-B for representative images). Counting was performed using sampling parameters sufficient to produce a Gunderson's coefficient of error (GCE, $\mathrm{m}=1$ ) of 0.12 or less. Stereo Investigator software used planar and depth information for each counted cell to calculate the volume for the digitally traced region of interest. Counting parameters were set to a counting frame of $60 \times 60 \mu \mathrm{m}^{2}$ and a dissector height of $15 \mu \mathrm{m}$ between the top and bottom guard zones. Estimated population number using weighted section thickness was recorded for each animal. The estimated cell count from two sections for each animal was averaged to give one datum point. Quantification is represented as an estimated total per mean measured thickness per $\mathrm{mm}^{3}$ to allow for comparisons across brain sections. An average of 5 animals per group were used in the analysis.

\subsubsection{Quantification of dendritic spines}

The brain hemispheres not processed for c-Fos or FosB were prepared for Golgi impregnation as previously described (Noye Tuplin, et al., 2018). For each of the three groups (control, chocolate flavored pellets, milk chocolate), nine secondary/tertiary dendrites on MSNs within the NAcSh per rat, with a minimum of 3 rats per abstinence period, were analyzed. This resulted in a minimum of 9 neurons per abstinence period within each group. The NAcSh was constricted in the anterior-posterior plane from approximately 1.7 to $3.2 \mathrm{~mm}$ from bregma according to Paxinos and Watson (1998). Neurons were picked at random but had to meet specific criteria to be selected: 1) neurons had to be entirely impregnated, 2) staining had to be uniform and complete, 3) the cell body had to be within the $200 \mu \mathrm{m}$ section depth, and 4) the neuron had to be relatively isolated from surrounding neurons. Neurons were reconstructed at 100X magnification using Neurolucida software (MicroBrightField, Williston, VT, USA). 
Three secondary/tertiary dendrites per rat were traced and spines were quantified based on type: thin, filopodia, stubby, and mushroom (see Figure 3.1C for representative image). For each neuron, the total number of spines was analyzed and divided by total dendritic length to obtain the NAcSh spine density for each rat. The measurements of the three analyzed neurons per rat were averaged to get one datum point per rat.

\subsubsection{Statistical analysis}

CPP preference scores were calculated to determine the degree of preference for the paired side compared to the unpaired side for each rat. Individual group CPP preference scores were analyzed using one sample t-tests with the test value set at $50 \%$. Preference scores were analyzed between groups with a fixed factor ANOVA with abstinence period and group (control, chocolate flavored pellet, milk chocolate) as independent variables. Within-group CPP preference scores and between group preference scores were also analyzed with repeated measures ANOVAs at two different time intervals, first ten minutes and last ten minutes, to determine whether the behavior showed any time dependence. One-way ANOVAs were used to determine differences in abstinence periods within groups. Side entries were analyzed using a multivariate ANOVA with paired and unpaired side as dependent variables and group and abstinence period as the independent variables. Tukey's post-hoc analyses were used if ANOVAs were significant. Alpha was set at 0.05 for significance.

FosB and c-Fos estimated cell counts were analyzed with fixed factor ANOVAs with abstinence period and food group as independent variables. Counts from the NAcC and NAcSh were analyzed separately. One-way ANOVAs were used to determine differences in abstinence periods within-groups and differences between groups at each abstinence period. Significant ANOVAs were followed up with Tukey's post-hoc analysis. Alpha was set at 0.05 for significance.

Dendritic spine density was analyzed using a within measures ANOVA with spine type as the within subjects measure and abstinence period and group as the between subjects factor. One-way ANOVAs were used to assess changes in dendritic spine density across abstinence periods within group and between groups at each abstinence period. Tukey's post-hoc analysis was used if one-way ANOVAs were significant. Alpha was set at 0.05 for significance.

\subsection{Results}

\subsubsection{Preference Testing}

Differences in preference ratio between food groups were analyzed using a fixed factor ANOVA with abstinence period and food group as the independent variables (Figure 3.2A) and revealed a main effect of food group $(\mathrm{F}(2,84)=4.200, \mathrm{p}<0.05)$ but no main effect of abstinence period $(\mathrm{F}(3,84)=0.185, \mathrm{p}>0.05)$ and no interaction $(\mathrm{F}(6,84)=$ $0.325, \mathrm{p}>0.05)$. Tukey's post-hoc analyses revealed that the chocolate pellet group spent significantly more time in the paired side compared to controls $(\mathrm{p}<0.05)$. 
A one-way ANOVA on abstinence period within the control group did not reveal a main effect of abstinence period $(\mathrm{F}(3,28)=1.033, \mathrm{p}>0.05)$ (Figure 3.2B). One-sample t-tests on preference ratio at each individual abstinence period within the control group revealed that time spent in the paired compared to unpaired side did not significantly differ $(\mathrm{p}>0.05)$.

A one-way ANOVA on abstinence period within the chocolate pellet group (Figure 3.2C) did not reveal a main effect of abstinence period $(\mathrm{F}(3,28)=0.595, \mathrm{p}>$ $0.05)$. One-sample t-tests on preference ratio at each individual abstinence period within the chocolate pellet group revealed that at the $24 \mathrm{hr}$. abstinence period rats spent significantly more time in the paired compared to unpaired side $(\mathrm{p}<0.05)$

A one-way ANOVA on abstinence period within the milk chocolate (Figure 3.2D) group did not reveal a main effect of abstinence period $(\mathrm{F}(3,28)=0.925, \mathrm{p}>0.05)$. Onesample t-tests on preference ratio at each individual abstinence period within the milk chocolate group revealed that time spent in the paired compared to unpaired side did not significantly differ $(\mathrm{p}>0.05)$.

Individual datum points were graphed to visually represent individual differences in preference ratios (Figure 3.2B-D). A preference can be defined as spending more than $50 \%$ of the time on the paired side and an aversion can be defined as spending less than $50 \%$ of the time on the paired side. For the chocolate pellet group (Figure 3.2C), at $24 \mathrm{hrs}$, the only time-period when there was a significant preference, 6 of the 8 rats spent more than $50 \%$ of the time in the paired side. In contrast, in the milk chocolate group (Figure 3.2D) only 3 of the 8 rats spent more than $50 \%$ of the 20 -minute test on the paired side with the other 5 spending more time on the unpaired side, with two rats showing an aversion of more than $75 \%$ of the time on the un-paired side.

To determine if a place preference was more likely to occur in the first or last 10minute interval of testing (Figure 3.3A), data were analysed with a repeated measures ANOVA. A repeated measures ANOVA with time as the within factor and abstinence period and food group as the between factors revealed a significant time $\mathrm{x}$ food group interaction $(\mathrm{F}(2,84)=3.392, \mathrm{p}<0.05)$.

The interaction was followed up with fixed factor ANOVAs on preference ratio at each time interval with abstinence period and food group as independent variables (Figure 3.3A). In the first 10-minutes of testing, there was no main effect of group $(\mathrm{F}(2,84)=1.179, \mathrm{p}>0.05)$, no main effect of abstinence period $(\mathrm{F}(3,84)=0.109, \mathrm{p}>$ $0.05)$, and no interaction $(\mathrm{F}(6,84)=1.029, \mathrm{p}>0.05)$. In the last 10 -minutes of testing, there was a main effect of group $(\mathrm{F}(2,84)=4.811, \mathrm{p}<0.05)$, but no main effect of abstinence period $(\mathrm{F}(3,84)=0.136, \mathrm{p}>0.05)$ and no interaction, $(\mathrm{F}(6,84)=0.736, \mathrm{p}>$ 0.05). A Tukey's post-hoc analysis on food group during the last 10-minutes of testing revealed that the chocolate pellet group spent significantly more time in the paired compartment compared to controls. $(\mathrm{p}<0.01)$.

A repeated measures ANOVA on the amount of time spent in the paired side for the control group with time (10-minute intervals) as the within factor and abstinence period as the between factor (Figure 3.3B) revealed that there were no main effects of 
time $(\mathrm{F}(1,28)=3.8, \mathrm{p}>0.05)$, no main effect of abstinence period $(\mathrm{F}(3,28)=1.014, \mathrm{p}>$ $0.05)$, and no interaction $(\mathrm{F}(3,28)=0.211, \mathrm{p}>0.05)$.

A repeated measures ANOVA on the amount of time spent in the paired side for the chocolate pellet group with time (10-minute interval) as the within factor and abstinence period as the between factor (Figure 3.3C) revealed that there were no main effects of time $(F(1,28)=3.332, p>0.05)$, no main effect of abstinence period $(F(3,28)=$ $0.834, \mathrm{p}>0.05)$, and no interaction $(\mathrm{F}(3,28)=0.919, \mathrm{p}>0.05)$. One-sample t-tests revealed that the chocolate pellet group spent significantly more time in the paired side compared to the unpaired side in the last ten minutes of testing following $24 \mathrm{hrs}$. and 7 days of abstinence $(\mathrm{p}<0.05)$.

A repeated measures ANOVA on the amount of time spent in the paired side for the milk chocolate group with time as the within factor and abstinence period as the between factor (Figure 3.3D) revealed that there was no main effect of time $(\mathrm{F}(1,28)=$ $1.428, \mathrm{p}>0.05)$, no main effect of abstinence period $(\mathrm{F}(3,28)=0.845, \mathrm{p}>0.05)$, and no interaction $(\mathrm{F}(3,28)=0.211, \mathrm{p}>0.05)$.

\subsubsection{Side Entries}

A fixed factor ANOVA on total side entries with abstinence period and food group as independent variables (Figure 3.4A) revealed a main effect of abstinence period $(F(3,84)=3.962, p<0.05)$, but no main effect of food group $(F(2,84)=0.221, p>0.05)$ and no interaction $(F(6,84)=1.377, p>0.05)$. A Tukey's post-hoc analysis on abstinence period revealed that at 7 days rats had significantly more total side entries compared to 28 days $(\mathrm{p}<0.01)$.

To determine the effect of abstinence period on total side entries within food groups, one-way ANOVAs on total side entries with abstinence period as the independent variable were performed (Figure 3.4A). In the control group, there was no main effect of abstinence period on total side entries $(\mathrm{F}(3,28)=1.975, \mathrm{p}>0.05)$. In the chocolate pellet group, a main effect of abstinence period was present for total side entries $(\mathrm{F}(3,28)=$ $4.713, \mathrm{p}<0.01)$. Tukey post-hoc analysis revealed that rats in the chocolate pellet group had significantly more side entries at 7 days compared to 14 and 28 days $(\mathrm{p}<0.05)$. In the milk chocolate group, there was no main effect of abstinence period on total side entries $(F(3,28)=0.435, p>0.05)$.

Multivariate analyses were used to determine if entries into the paired and unpaired side differed between abstinence periods within each food group. A multivariate analysis with abstinence period as the independent variable revealed that in the control group (Figure 3.4B) paired $(F(3,28)=1.417, p>0.05)$ and unpaired entries $(F(3,28)=$ $1.982, \mathrm{p}>0.05)$ did not significantly differ across abstinence periods.

For the chocolate pellet group (Figure 3.4C), a multivariate analysis with abstinence period as the independent variable revealed a main effect of paired $(\mathrm{F}(3,28)=$ $4.069, \mathrm{p}<0.01)$ and unpaired entries $(\mathrm{F}(3,28)=3.802, \mathrm{p}<0.05)$. Tukey's post-hoc analysis on paired entries revealed that rats entered the paired side significantly more at the 7-day abstinence period compared to the 14 and 28 -day periods $(\mathrm{p}<0.05)$. Tukey's 
post-hoc analysis on unpaired entries revealed that rats entered the unpaired side significantly more at 7 days compared to 28 days $(\mathrm{p}<0.05)$.

For the milk chocolate group (Figure 3.4D), a multivariate analysis with abstinence period as the independent variable revealed there was no significant difference in paired $(F(3,28)=0.721, p>0.05)$ and unpaired entries $(F(3,28)=0.226, p>0.05)$ between abstinence periods.

Paired sample t-tests were performed to examine if rats spent more time entering the paired vs. unpaired compartments during the preference test. In the control group, there was no significant difference between paired and unpaired side entries $(\mathrm{t}(31)=$ $0.741, \mathrm{p}>0.05$ ) (Figure 3.4B. In the chocolate pellet group rats entered the paired side significantly more times compared to the unpaired side $(t(31)=2.697, p<0.05)$ (Figure $3.4 \mathrm{C})$. In the milk chocolate group, there was no significant difference between paired and unpaired side entries $(\mathrm{t}(31)=0.272, \mathrm{p}>0.05)$ (Figure 3.4D). Paired-sample t-tests at each individual abstinence periods within food group did not reveal any significant differences between paired and unpaired sides $(\mathrm{p}>0.05)$.

\section{4 .3 c-Fos}

A fixed factor ANOVA on c-Fos labelling in the NAcSh with abstinence period and food group as the independent variables (Figure 3.5A; 3.5C for representative images) revealed a significant interaction between abstinence period and food group $(F(6,54)=2.504, p<0.05)$, but no main effect of food group $(F(2,54)=0.171, p>0.05)$, and no main effect of abstinence period $(\mathrm{F}(3,54)=1.589, \mathrm{p}>0.05)$. One-way ANOVAs at each abstinence period revealed a main effect of food group at 7 days $(F(2,18)=3.823$, $\mathrm{p}<0.05)$. A Tukey's post-hoc analysis revealed that at the 7-day abstinence period the chocolate pellet group had significantly more c-Fos labeling compared to the control group $(\mathrm{p}<0.05)$. One-way ANOVAs on abstinence period within each food group revealed a main effect of abstinence period in the NAcSh in the control group $(\mathrm{F}(3,22)=$ $3.337, \mathrm{p}<0.05)$. Tukey's post-hoc analysis revealed that there was significantly more cFos labeling in the control group at the 28-day abstinence period compared to 7 days $(\mathrm{p}<$ $0.05)$.

A fixed factor ANOVA on c-Fos labelling in the NAcC with abstinence period and food group as the independent variables (Figure 3.5B; 3.5C for representative images) did not reveal a significant interaction $(\mathrm{F}(6,54)=2.009, \mathrm{p}>0.05)$, and no main effect of food group $(\mathrm{F}(2,54)=0.660, \mathrm{p}>0.05)$ or abstinence period, NAcC: $(\mathrm{F}(3,54)=$ $1.294, \mathrm{p}>0.05)$. There were no main effects of food group at each individual abstinence period and no main effects of abstinence period within each food group.

In the control group (Figure 3.6A-B), a significant negative correlation between the amount of food consumed the day before testing and c-Fos labelling in the NAcSh, $\mathrm{r}(22)=-0.634, \mathrm{p}<0.01$, and NAcC, $\mathrm{r}(22)=-0.629, \mathrm{p}<0.01$ was noted. The correlation between c-Fos expression and food consumption on the day before testing was not significant in the chocolate pellet group (NAcSh; $r(22)=-0.032, p>0.05$ NAcC; $r(22)=$ $-0.153, \mathrm{p}>0.05$ ) (Figure 3.6C-D) or the milk chocolate group (NAcSh; $r(22)=-0.032, p$ $>0.05$ NAcC; $r(22)=-0.117, \mathrm{p}>0.05)($ Figure 3.6E-F). 


\subsubsection{FosB}

A fixed factor ANOVA on FosB labeling in the NAcSh with abstinence period and food group as independent variables (Figure 3.6A; 3.6C for representative images) revealed a main effect of abstinence period $(\mathrm{F}(3,52)=2.837, \mathrm{p}<0.05)$, but no main effect of food group $(\mathrm{F}(2,52)=1.965, \mathrm{p}>0.05)$ and no interaction $(\mathrm{F}(6,52)=1.434, \mathrm{p}>$ $0.05)$. One-way ANOVAs on each abstinence period with food group as the independent variable revealed a main effect of food group at 7 days $(F(2,11)=11.841, p<0.01)$. Tukey's post-hoc analysis revealed that the chocolate pellet group had significantly more labelled cells compared to the milk chocolate $(\mathrm{p}<0.05)$ and control groups $(\mathrm{p}<0.05)$. There were no main effects of abstinence period within each food group.

A fixed factor ANOVA on FosB labeling in the NAcC with abstinence period and food group as independent variables (Figure 3.6B; 3.6C for representative images) did not reveal a main effect of abstinence period $(F(3,52)=1.386, p>0.05)$, food group $(\mathrm{F}(2,52)=0.981, \mathrm{p}>0.05)$, and no interaction $(\mathrm{F}(6,52)=1.481, \mathrm{p}>0.05)$. One-way ANOVAs on each abstinence period with food group as the independent variable revealed a main effect of food group at 7 days $(F(2,11)=4.577, p<0.05)$. Tukey's posthoc analysis revealed that the chocolate pellet group had significantly more labelled neurons compared to the control group $(\mathrm{p}<0.05)$. One-way ANOVAs within each food group with abstinence period as the independent variable revealed a main effect of abstinence period in the control group $(\mathrm{F}(3,18)=3.392, \mathrm{p}<0.05)$. Tukey's post-hoc analysis revealed that the 14-day abstinence period was associated with significantly higher FosB labelling compared to 7 days. There were no main effects of abstinence period in the chocolate pellet or milk chocolate groups.

\subsubsection{Dendritic Spine Density}

A fixed factor ANOVA on total spines in the NAcSh with abstinence period and food group as independent variables (Figure 3.8A; 3.10 for representative images) did not reveal any main effect of abstinence period $(\mathrm{F}(3,24)=1.912, \mathrm{p}>0.05)$ or food group $(\mathrm{F}(2,24)=0.126, \mathrm{p}>0.05)$ and there was no interaction $(\mathrm{F}(6,24)=1.687, \mathrm{p}>0.05)$. Oneway ANOVAs on each individual abstinence period with food group as the independent variable revealed a main effect of food group at the 28-day abstinence period $(\mathrm{F}(2,6)=$ $7.204, \mathrm{p}<0.05)$. Tukey's post-hoc analysis revealed that the chocolate pellet group had a significantly higher total spine density compared to the milk chocolate group $(\mathrm{p}<0.05)$ (control; $p=0.064)$. One-way ANOVAs within each food group with abstinence period as the independent variable revealed a main effect of abstinence period for the control group $(\mathrm{F}(3,8)=4.140, \mathrm{p}<0.05)$. Tukey's post-hoc analysis revealed that there was a significantly higher spine density at the 14-day abstinence period compared to 28 days ( $p$ $<0.05)$

Spine analysis was broken down into four different spine types, including thin (Figure 3.8B), mushroom (Figure 3.8C), stubby (Figure 3.8D), and filipodia (figure 3.8E). Fixed factor ANOVAs on each spine type in the NAcSh with abstinence period and food group as independent variables were performed. None of the fixed factor ANOVAs revealed significant main effects of abstinence period, food group, or any 
interactions within any of the individual spine types. Separate one-way ANOVAs on each spine type on individual abstinence periods with food group as the independent variable revealed a main effect of food group for thin spines at the 28-day abstinence period $(\mathrm{F}(2,6)=12.636, \mathrm{p}<0.01)$. Tukey's post-hoc analysis revealed that the milk chocolate group has significantly lower thin dendritic spine density compared to the control $(\mathrm{p}<0.05)$ and chocolate pellet groups $(\mathrm{p}<0.01)$. Separate one-way ANOVAs on each spine type on abstinence period within each food type did not revealed any significant difference in spine density across abstinence periods for any food group.

A fixed factor ANOVA on total spines in the NAcC with abstinence period and food group as independent variables (Figure 3.9A) did not reveal any main effect of abstinence period $(\mathrm{F}(3,24)=2.599, \mathrm{p}>0.05)$ or food group $(\mathrm{F}(2,24)=0.088, \mathrm{p}>0.05)$ and there was no interaction $(\mathrm{F}(6,24)=1.077, \mathrm{p}>0.05)$. One-way ANOVAs on each individual abstinence period with food group as the independent variable did not reveal any main effects of group. One-way ANOVAs within each food group with abstinence period as the independent variable did not reveal any main effects of abstinence period in any of the food groups.

Spine analysis was broken down into four different spine types, including, thin (Figure 3.9B), mushroom (Figure 3.9C), stubby (Figure 3.9D), and filipodia (figure 3.9E). Fixed factor ANOVAs on each spine type in the NAcC with abstinence period and food group as independent variables were performed. None of the fixed factor ANOVAs revealed significant main effects of abstinence period, food group, or any interactions within any of the individual spine types. Separate one-way ANOVAs on each spine type on individual abstinence periods with food group as the independent variable did not reveal any main effects. Separate one-way ANOVAs on each spine type on abstinence period within each food type did not revealed any significant difference in spine density across abstinence periods for any food group.

\subsection{Discussion}

In the present study, neither the chocolate pellet nor milk chocolate groups showed an increase in their preference ratio at any of the four abstinence periods tested. The chocolate pellet group displayed a preference for the paired side at the 24-hr. abstinence period and at the 7-day abstinence period, which was most prominent in the last ten minutes of testing. Overall, the chocolate pellet group spent significantly more time in the paired side compared to the control group, confirming the rewarding effect of the pellets. Unexpectedly, the milk chocolate group did not display a preference for the paired side at any time point and over half of the rats that consumed milk chocolate displayed an aversion at the 24-hr. time point. Overall, the milk chocolate group did not significantly differ from the control group.

Incubation of craving has been consistently reported in self-administration models using drugs of abuse and natural reinforcers (Grimm et al., 2001, 2002; Li and Frantz, 2009). When non-contingent models have been utilized, such as the CPP model, the effect is less consistent. For example, in non-contingent designs both time-dependent increases (Li et al., 2008; Lubbers et al., 2015) and decreases (Sun et al., 2017) in CPP 
scores have been shown following morphine (Li et al., 2008; Sun et al., 2017) and cocaine abstinence (Lubbers et al., 2015). The main difference between the studies was that in the study that did not display an incubation of craving response, the same rats were tested at each abstinence period (Sun et al., 2017). In the studies that did display a CPP response, rats were only tested at one abstinence period (Li et al., 2008; Lubbers et al., 2015).

One explanation for the inability of milk chocolate to induce a CPP may be individual differences between rats, with some rats showing strong aversions (under $25 \%$ preference ratio) or preferences (over $75 \%$ preference ratio). Individual differences in locomotor sensitization, response to novelty and craving are well documented with respect to methamphetamine (Bevins et al., 1997), cocaine (Mandt et al., 2009), and food (Gearhardt et al., 2014; Schüz et al., 2015). Rats trained to self-administer cocaine can be classified as either high or low responders based on the median split of an open field locomotor test during the 30 minutes following a single dose of cocaine $(10 \mathrm{mg} / \mathrm{kg})$ (Mandt et al., 2009). Response to novelty may also play a role in individual differences in responding, where high responders to novelty are more sensitive to the bar-pressing suppressant effects of amphetamine (Bevins et al., 1997). In humans, individual differences in craving and liking between foods, such as sugar or fat, are common and may play a role in problematic eating behavior (Gearhardt et al., 2014). In the present study we did not develop a categorization that accounted for individual differences in preference for either the chocolate flavored pellets or milk chocolate chips, which may have played a role in the variability within both the milk chocolate and chocolate pellet groups.

It has been suggested that entries into the paired compartment may represent seeking behavior which can be distinguished from the reward memory measured with the preference score (Duarte et al., 2014; Sun et al., 2017). In the present study, there were no overall group differences between entries but there was a significant interaction between food type and entries into the paired side and unpaired side. Overall, the chocolate pellet group entered the paired side significantly more times than the unpaired side. When entries were examined over the abstinence periods, the chocolate pellet group displayed the highest number of side entries at 7 days before decreasing at 14 and 28 days, suggesting food seeking decreased over time. A study by Sun and colleagues (2017) found similar results when investigating the incubation of craving for morphine in a CPP model (Sun et al., 2017). After conditioning, it was found that rats showed a preference for the morphine paired side following 2 and 10 days of abstinence followed by a decrease in the CPP scores. The morphine-trained rats showed an increase in side entrances over time, which was not evident in the saline-treated rats. The authors suggested that reward memory (preference score) and drug craving (entries) could be distinguished in their CPP model (Sun et al., 2017).

The decrease in preference score over the abstinence periods in the chocolate pellet group is similar to what has been found with other CPP studies using food as a reward (Duan et al., 2016; Orsini et al., 2013). Orsini and colleagues (2013) investigated the occurrence of a CPP for cocaine or milk chocolate following an extinction session (Orsini et al., 2013). Mice were tested for the CPP at 1 or 9 days following the extinction test. One day following the extinction test, mice continued to show a preference for the 
compartment previously paired with chocolate but by day 9 , mice no longer showed a preference for the chocolate-paired cues but continued to show a preference for the cocaine-paired cues (Orsini et al., 2013). Duan and colleagues (2016) tested for a CPP following 1, 14 or 28 days of forced abstinence from food reward (apple) or morphine (Duan et al., 2016). The morphine-treated animals continued to spend significantly more time in the morphine paired compartment at the 28 day abstinence period, whereas the food group did not display a preference after the 28 day abstinence period (Duan et al., 2016).

Food restriction may have played a role in the limited expression of CPP in the present study. Non-homeostatic eating behavior has been compared to drug abuse (Corwin and Hajnal, 2005). Behaviors that are non-homeostatic are often excessive behaviors that involve overconsumption of food or drugs (Corwin and Hajnal, 2005). Food restriction puts animals into a state of caloric deficit where food seeking behavior becomes homeostatic and so food seeking behavior may be independent from food palatability (Beeler et al., 2012). In the present study, food restriction was maintained throughout the study which may have resulted in homeostatic food seeking behavior, rather than non-homeostatic, mechanisms.

The quantity of reward and amount of conditioning sessions may play a role in the development, magnitude, and persistence of the CPP (Alderson et al., 2001; Brabant et al., 2005). When rats were tested for a CPP to $4 \%, 12 \%$, or $20 \%$ sucrose solution, rats did not develop a CPP for the 4\% solution (Alderson et al., 2001). One conditioning session has been shown to be insufficient to induce a CPP to 4,8 or $12 \mathrm{mg} / \mathrm{kg}$ of cocaine but two or four sessions produced a similar magnitude of responding across doses (Brabant et al., 2005). However, in rats conditioned for two sessions, the CPP lasted for less than 14 days at all doses, but in the four session groups, the CPP was still present at 28 days and the magnitude was dose dependent (Brabant et al., 2005). The present study employed $4 \mathrm{~g}$ of chocolate flavored pellets or milk chocolate chips on the paired side. This is similar to the quantity of reward used in a study by Baunez and colleagues (2005) which investigated CPP for sucrose pellets ( $4.5 \mathrm{~g} ; 45 \mathrm{mg}$ each) in food restricted rats that resulted in a preference for the paired side $24 \mathrm{hrs}$ after training (Baunez et al., 2005). The present study measured the CPP up to 28 days after abstinence, which differed from the Baunez study which only measured CPP following $24 \mathrm{hrs}$ of abstinence, so it is possible that the quantity of conditioning for reward may still be a factor in the present study.

\subsection{1 c-Fos}

In studies on the incubation of craving, c-Fos labeling is elevated in the NAcSh and NAcC following 30 days of abstinence from sucrose (Grimm et al., 2016). In the present study, the control group displayed significantly more c-Fos labelling at 28 days compared to 7 days, but neither of the food groups displayed a time-dependent change in c-Fos labeling. Food restriction alone has been shown to increase c-Fos labelling within the NAc (Mendoza et al., 2005). Level of food restriction may have contributed to the elevated c-Fos labeling in the control group as higher levels of c-Fos labeling were correlated with less food as required to maintain the restricted weights. As the amount of food given to maintain weight was decreased, c-Fos expression increased in the control group; an effect that was not significant within the two chocolate groups. Neither 
chocolate group had a significant decline in the amount of food necessary to maintain weight over the abstinence periods. This could have contributed to the lack of abstinence period effect in these food groups.

Cues that have acquired an incentive value linked with rewarding foods have been shown to be associated with elevated c-Fos labeling in the NAc (Figlewicz et al., 2011; Flagel et al., 2011). In the current study, there were no significant differences between groups at the $24 \mathrm{hr}, 14$ day, or 28-day abstinence periods. Food restriction may have played a role in the lack of differences between food groups. In behavioral studies, rats are often food restricted to provide motivation for food-seeking, which may sensitize the mesocorticolimbic pathway, as evidenced by increased sensitivity to DA agonists and compulsive seeking behavior for food and drugs (Patrono et al., 2015). The increase in cFos expression associated with food restriction has been suggested to result from increased DA receptor signaling (Carr et al., 2003).

Food anticipation, along with food restriction, can influence c-Fos expression in the NAc (Blancas et al., 2014). Anticipation of feeding in animals that are under food restriction to normal chow has been shown to result in elevated c-Fos in the NAc (Mendoza et al., 2005). In the present study, animals in all food groups were food restricted and were fed chow at approximately the same time every day, thus the control group labeling could also have been influenced by food anticipation. This may also contribute to the lack of group differences at the 24-hr. abstinence period where the chocolate pellet group did display a place preference. Testing occurred on the day after rats underwent their final conditioning session for chocolate. During conditioning days, this would indicate they would not receive chocolate the next day. Thus, rats may not have been anticipating palatable food at the 24-hr. testing period.

At the 7-day abstinence period, the chocolate pellet group displayed significantly more c-Fos labeling than the control group, but not the milk chocolate group, in the NAcSh. The elevated c-Fos labeling in the chocolate pellet group coincides with the abstinence period when entries are the highest in the chocolate pellet group. The chocolate pellet group was the only group to display a CPP at this abstinence period, specifically in the last ten minutes of testing. Increased c-Fos has been associated with drug seeking behavior in response to drug associated cues (Cruz et al., 2015). Since the chocolate pellet group displayed its highest number of entries at the 7-day period, it is probable that c-Fos expression was associated with the seeking behavior. It is not surprising that there were no significant differences between groups at the 14-day and 28day abstinence periods as none of the groups displayed a CPP at these time points. The lack of difference between the chocolate pellet group and milk chocolate group may be the result of individual differences between preference and aversion for each food. Unlike taste preference, taste aversion is not associated with changes in c-Fos staining within the NAc (Yasoshima et al., 2006).

The type of reward has been shown to play a role in c-Fos induction in the NAc. There are similarities between food and drug reward in relation to seeking behavior (Corwin and Hajnal, 2005; Rogers and Smit, 2000) but differences have been shown for c-Fos expression (Koya et al., 2006; Schroeder et al., 2001). For example, rats that underwent CPP for either nicotine or chocolate chips displayed different c-Fos expression 
patterns (Schroeder et al., 2001). In association with contextual cues previously paired with nicotine, there was an increase in c-Fos expression in the NAcSh whereas exposure to chocolate associated contextual cues did not result in an increase (Schroeder et al., 2001). In another study, rats that underwent self-administration for heroin showed elevated c-Fos expression in the NAcC during a responding for cues test but rats that were trained to self-administer sucrose did not show this elevation (Koya et al., 2006). In the present study, the food types did not significantly differ from each other at any abstinence period and only the chocolate pellet group significantly differed from the control group at the 7-day abstinence period. It is possible that the rewarding properties of these foods were not sufficient to induce c-Fos expression above a food restricted control level.

\subsubsection{FosB}

In the present study, food restricted controls displayed similar FosB labelling at the $24 \mathrm{hr}, 14$ day, and 28-day abstinence periods as rats that received chocolate pellets or milk chocolate chips. At the 7-day abstinence period, the chocolate pellet group displayed significantly more FosB labelling than the control group in both the NAcSh and NAcC. The level of FosB labeling did not change over the abstinence periods in the chocolate pellet group or milk chocolate groups in the NAcSh or NAcC. DeltaFosB has a half-life of weeks to months following rewarding substances, thus this is unsurprising (Nestler et al., 2001). The lack of differences between groups at the 14 day and 28-day abstinence periods coincides with the lack of group differences in CPP.

Chronic exposure to both food (Stamp et al., 2008) and drugs (Larson et al., 2010) has been shown to be associated with DeltaFosB accumulation but accumulation of DeltaFosB over periods of abstinence has been shown to differ between food (Sharma et al., 2013) and drugs (Harris et al., 2007). For example, DeltaFosB has been shown to accumulate during abstinence in animals that have been exposed to chronic cocaine administration (Harris et al., 2007) which has been shown to continue for three and five weeks of abstinence (Harris et al., 2007). In the NAc, exposure to high fat, but not low fat, food following conditioning in a CPP study resulted in increased DeltaFosB expression (Sharma et al., 2013). Following abstinence from high fat food, DeltaFosB decreased rapidly, whereas following abstinence from low fat food there was no change in DeltaFosB expression (Sharma et al., 2013). In the present study, we did not measure FosB expression immediately after conditioning so we cannot determine if there was a decrease in FosB following abstinence. We did observe consistent FosB labeling over the abstinence periods in both chocolate groups, but there were limited behavioral effects so the potential effect of food restriction on FosB labeling cannot be discounted.

The labelling of different FosB isoforms could be a factor in the lack of group differences at all but the 7-day abstinence period. The FosB antibody labels cells for both FosB, and the more stable isoform DeltaFosB, thus there is no way to differentiate between the two. The less stable isoform, FosB, reduces to baseline approximately 6 hours following acute stimulation whereas the DeltaFosB isoform is stable for weeks (Nestler et al., 2001). Drug exposure has been shown to induce both FosB and DeltaFosB within the NAcSh and NAcC but the effect for FosB is diminished by $24 \mathrm{hrs}$. 
whereas the effect of DeltaFosB continues (Larson et al., 2010). In the present study, we did not investigate FosB labeling in animals that did not undergo testing, thus the contribution of FosB and DeltaFosB to the overall FosB labelling is unknown. Differences between the chocolate pellet group and control group were present in both the NAcSh and NAcC at the 7-day abstinence period. This is similar to drug studies that have shown prolonged DeltaFosB labeling in both NAc regions following abstinence from chronic cocaine administration (Larson et al., 2010). A lack of group differences could also be the result of acute FosB induction during exposure to the apparatus itself.

\subsubsection{Dendritic Spine Density}

Long-term changes in dendritic spine density has been correlated with substance seeking behaviors (Robinson and Kolb, 2004). Dendritic spine density in the NAc has been shown to be altered in incubation of craving studies following self-administration of high fat food or chow in both the NAcSh and NAcC (Dingess et al., 2017b) and following self-administration of cocaine in the NAcC (Christian et al., 2016). In the NAcSh, abstinence from chow following self-administration has been associated with an increase in thin spines following one day of abstinence and mushroom spines following 30 days of abstinence; however, the high fat group did not differ from control (Dingess et al., 2017b). In the NAcC, the high fat group also displayed elevated mushroom spine density compared to control at the 30-day abstinence period (Dingess et al., 2017b). This highlights the ability of different food types, of different macronutrient composition, to elicit the same behavioral response but display differences at the synaptic level (Dingess et al., 2017b).

The formation of thin spines can be rapid, whereas mushroom type spines are the result of longer-term changes (Bourne and Harris, 2007; Huber and Menzel, 2004). In the present study, there were significantly more thin spines at the 28-day abstinence period in the control and chocolate pellet groups compared to the milk chocolate group Control rats were exposed to the apparatus for the same length of time as rats that received chocolate, so this could be a factor in the lack of group differences. Since thin spines can be formed rapidly, it is possible that exposure to the apparatus itself resulted in spine formation. In the milk chocolate group, some rats displayed an aversion which may have contributed to the lower thin spine density compared to control rats.

The chocolate pellet group also displayed a higher total spine density compared to both milk chocolate and control rats. We have previously shown that consumption of chocolate flavored pellets in an operant task resulted in a prolonged increase in total dendritic spine density in the NAcSh compared to food restricted controls (Noye Tuplin et al., 2018). The total spine density in the chocolate pellet group remained steady over time, whereas in the control group, total spine density dropped between the 14 day and 28-day abstinence periods. This raises the possibility that the consumption of chocolate flavored pellets may have contributed to long-term changes in the NAcSh that are persistent. Since the milk chocolate group did not display a CPP, it follows that spine density was significantly lower than the chocolate pellet group, and similar to control, at the 28-day abstinence period. 
It is not unusual for spine changes to not manifest during early periods of abstinence. Following chronic cocaine self-administration, dendritic spine density did not differ from control levels until 36 days of forced abstinence (Christian et al., 2016). For cocaine, the dendritic spine density was approximately the same following 14 and 36 days of abstinence, but by day 36 , the dendritic spine density in the saline group decreased (Christian et al., 2016). This is a similar pattern that was seen in the present study following 28-days of abstinence from chocolate pellets. The authors suggested that at day 36, calcium permeable AMPA receptors, which are shown to be elevated in the incubation of craving and can affect structural plasticity (Conrad et al., 2008), are maximally elevated and affected spine density (Christian et al., 2016).

\subsection{Conclusions}

The presented behavioral model was not conducive in modeling incubation of food craving on a neural or behavioral level. We have shown that the type of food reward may be an important factor in eliciting a robust CPP response. Greater within group variability in rats conditioned for milk chocolate suggests that certain foods may have a greater propensity to be like or disliked. The chocolate pellet group was the only group to differ from control in the labelling of c-Fos and FosB and it was only significant at the 7-day abstinence period. This coincided with the time period when the chocolate pellet group had the most side entries, thus immediate early gene labelling may be associated with food seeking. At the 28-day abstinence period, the chocolate pellet group displayed significantly more spines overall compared to the control and milk chocolate groups, but there were no significant behavioral effects at this time point. This may reflect long-term changes in reward circuitry but the functional significance of this remains elusive. Chocolate pellets produced short-term seeking behavior but, as evidenced by limited neural changes, these food rewards may not be sufficient to induce enhanced craving following long periods of forced abstinence. 


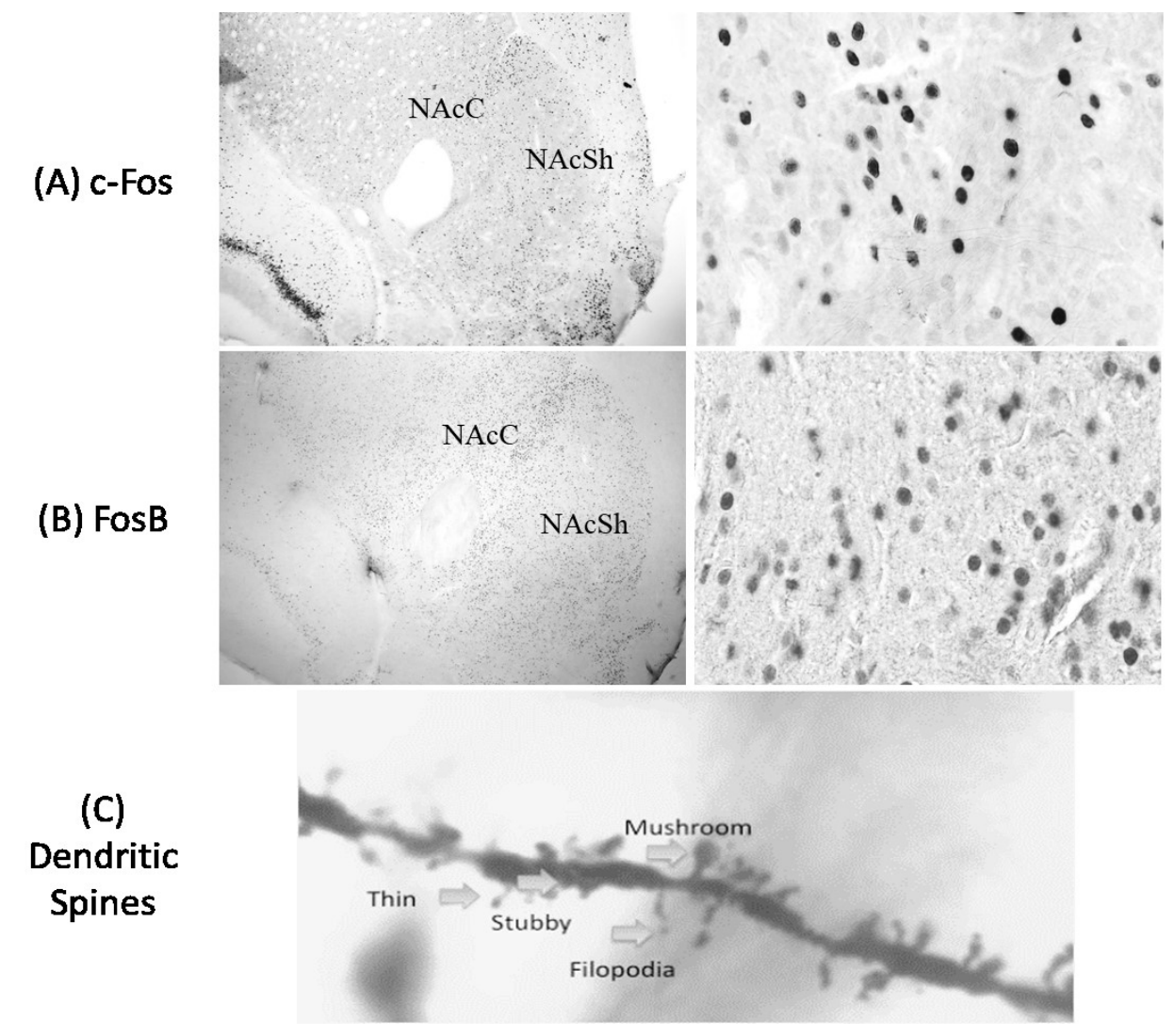

Figure 3.1. Representative images of immunohistochemically labelled neurons and spine classification. (A) Representative image of c-Fos labelled cells in the NAcSh and NAcC 4x and labelled cells at 60x. (B) Representative image of FosB labelled cells in the NAcSh and NAcC 4x and labelled cells at 60x. (C) Representative image of dendritic spine classification on MSNs in the NAc 100x. 
(A)

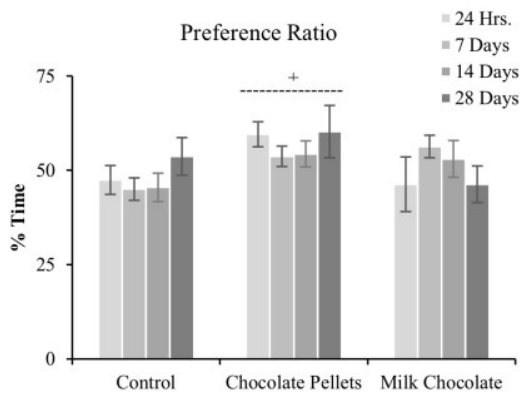

(C)

Chocolate Pellets

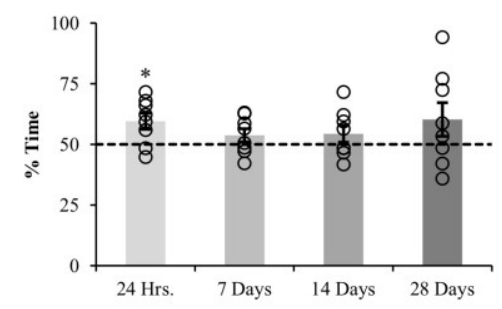

(B)

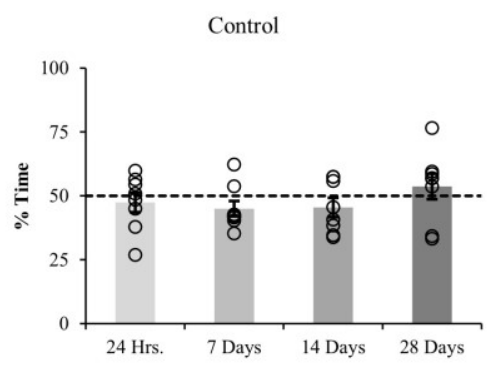

(D)

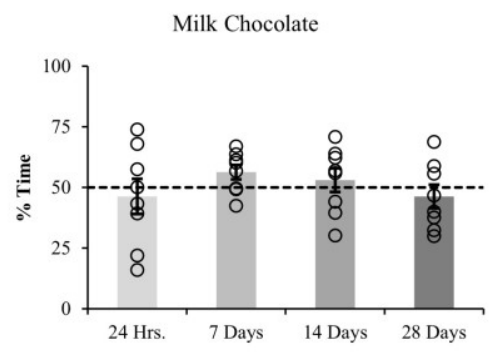

Figure 3.2. Preference ratio during the 20-minute test (Mean \pm SEM). (A) Comparison between food groups independent of abstinence period. $+p<0.05$ compared to the control group. (B) Control group individual preference scores for each abstinence period. (C) Chocolate Pellet group individual preference scores for each abstinence period. ${ }^{*} \mathrm{p}<0.05$ compared to time spend in the unpaired compartment. (D) Milk chocolate group individual preference scores for each abstinence period 


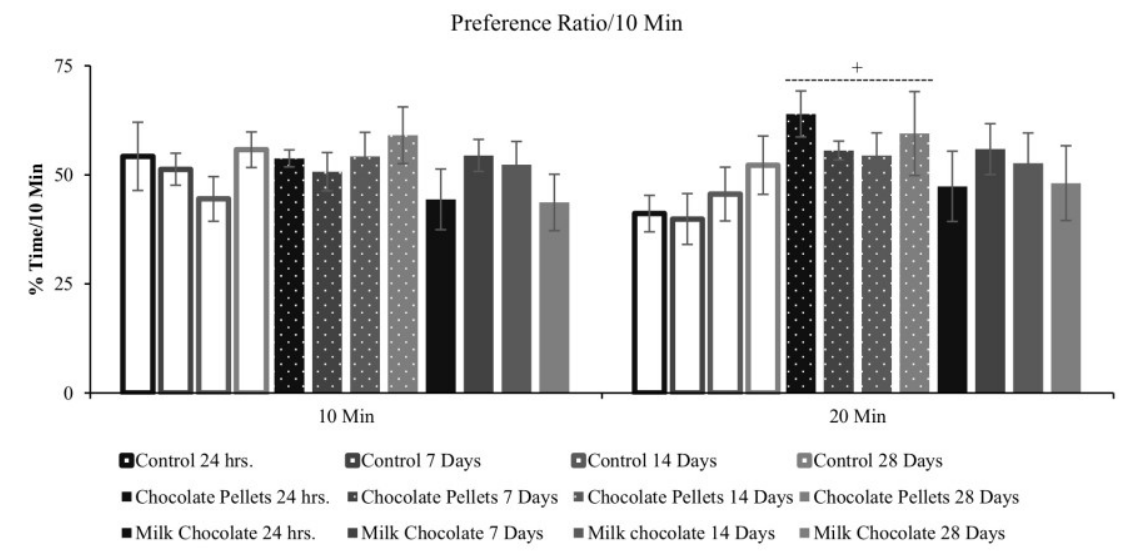

(B)

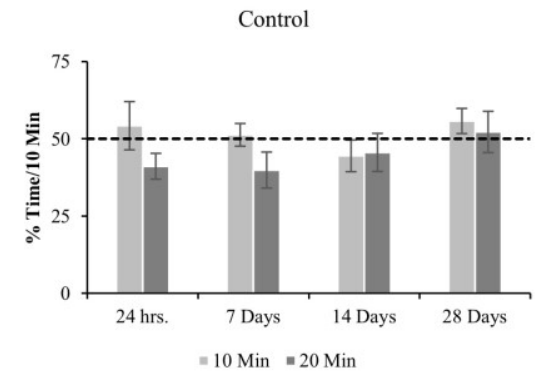

(D)

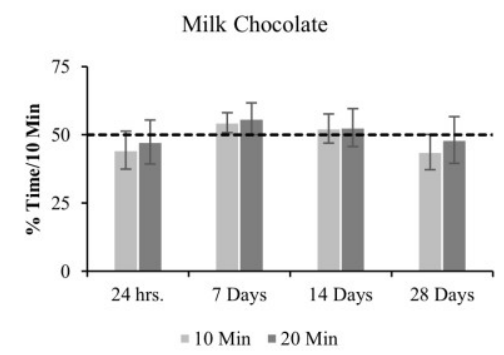

(C)

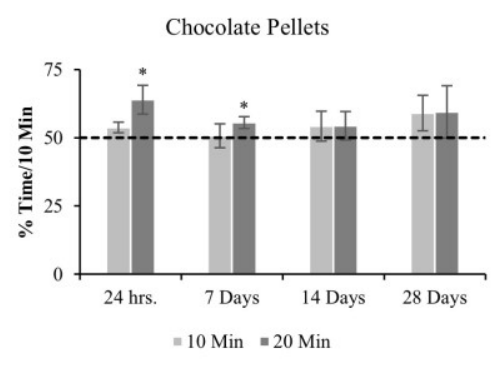

Figure 3.3. Preference scores per 10-minutet testing interval (mean \pm SEM) (A) Preference scores per 10minute interval for all groups. $+p<0.05$ compared to control group at same time interval (B) Control group across each abstinence period. (C) Chocolate pellet group across each abstinence period. ${ }^{*} \mathrm{p}<0.05$ compared to unpaired compartment. (D) Milk Chocolate group across each abstinence period. 
(A)

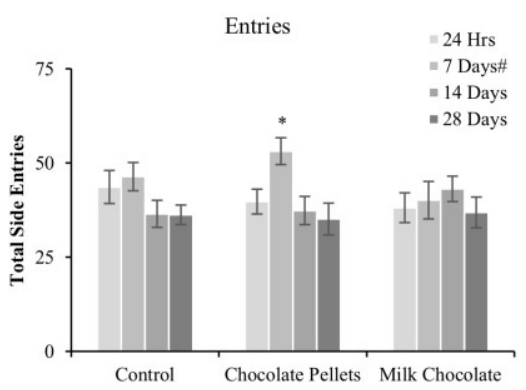

(C)

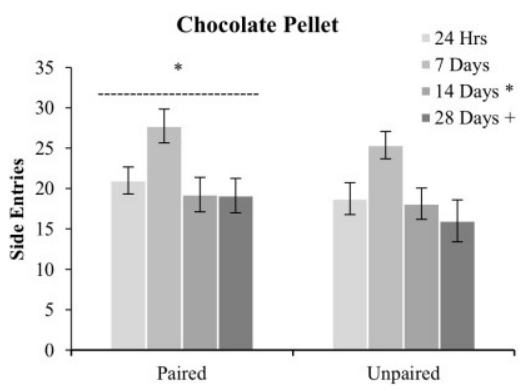

(B)

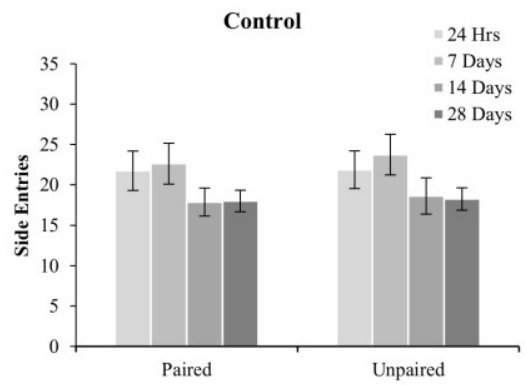

(D)

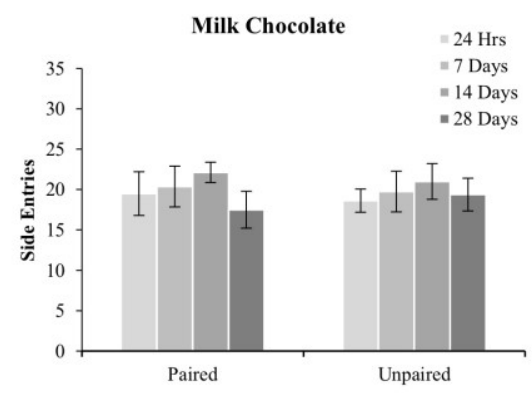

Figure 3.4. Side Entries (Mean \pm SEM). (A) Total side entries (Paired + Unpaired). ${ }^{*} \mathrm{p}<0.05$ compared to the 14 and 28-day abstinence periods within food group. $\# \mathrm{p}<0.01$ compared to 28 -day abstinence period. (B) Control group paired vs unpaired side entries. (C) Chocolate pellet group paired vs unpaired side entries. ${ }^{*} \mathrm{p}<0.05$ compared to unpaired side entries. $* \mathrm{p}<0.05$ compared to paired entries at the 7 -day abstinence period. $+p<0.05$ compared to paired and unpaired entries at the 7-day abstinence period (D) Milk chocolate paired vs unpaired side entries. 
(A)

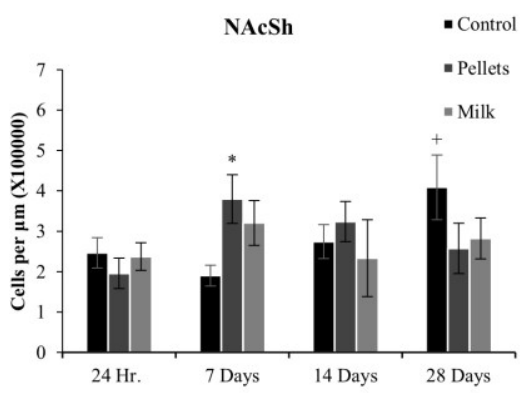

(B)

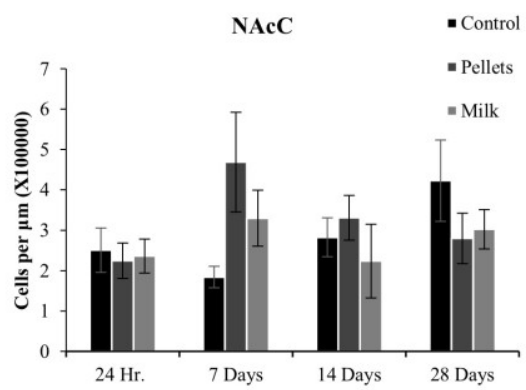

(C)

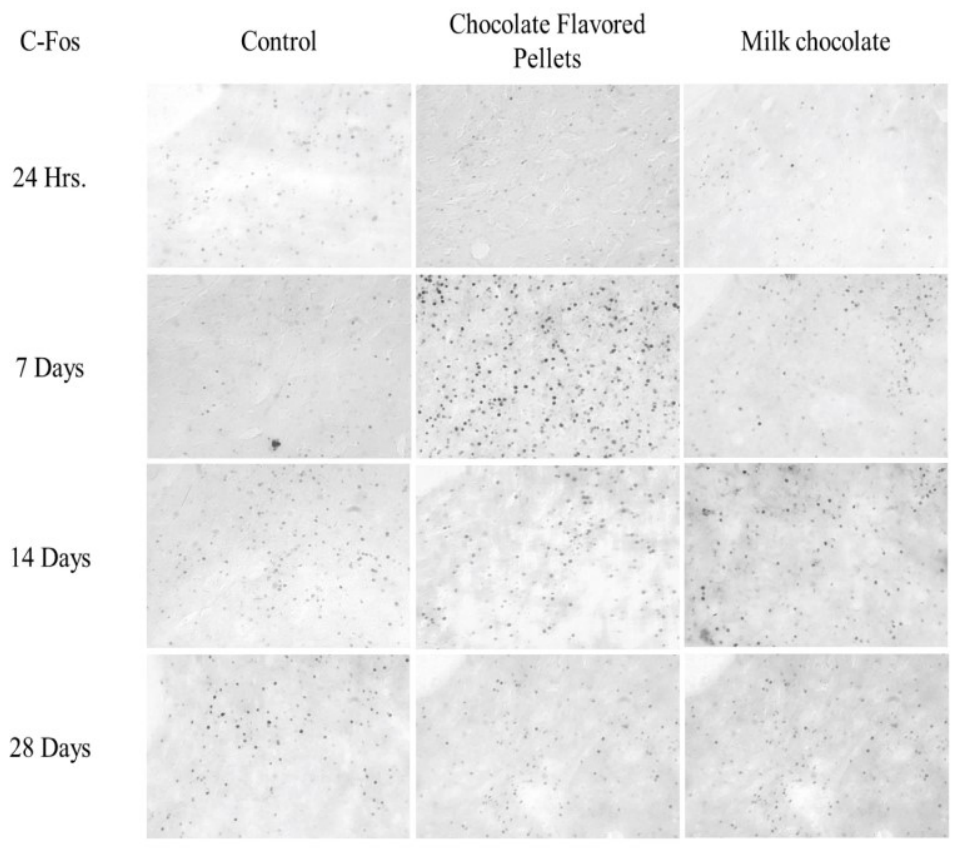

Figure 3.5. Average cell density (mean cell count/volume $\left[\mathrm{mm}^{3}\right]$ ) of c-Fos labeled neurons (mean $\pm \mathrm{SEM}$ ) (A) NAcSh labeled neurons in rats that underwent CPP for food reward compared to control. ${ }^{*} \mathrm{p}<0.05$ compared to control at same abstinence period. ${ }^{+} \mathrm{p}<0.05$ compared to control at 7-day abstinence period. (B) NAcC labeled neurons in rats that underwent CPP for food reward compared to control. (C) Representative images of c-Fos labelling in the NAc. 20x. 
(A)

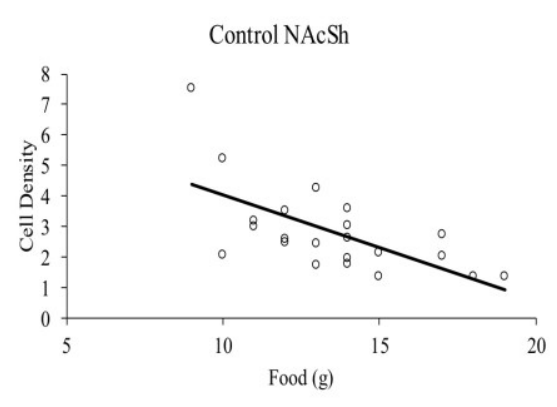

(B)

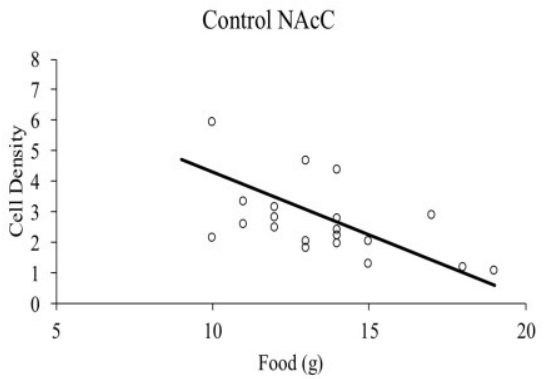

(C)

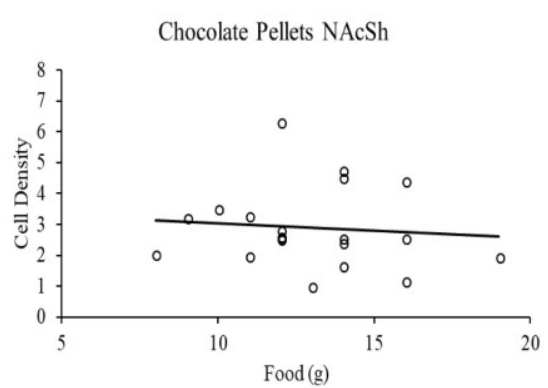

(D)

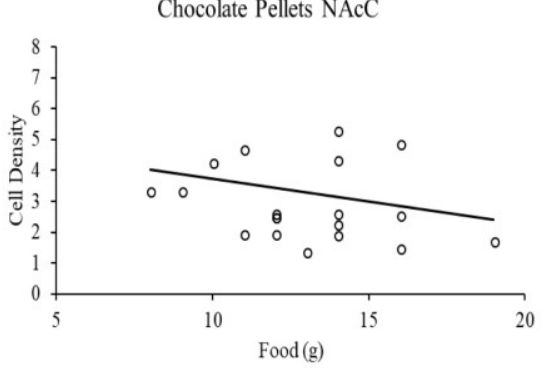

(E)

(F)
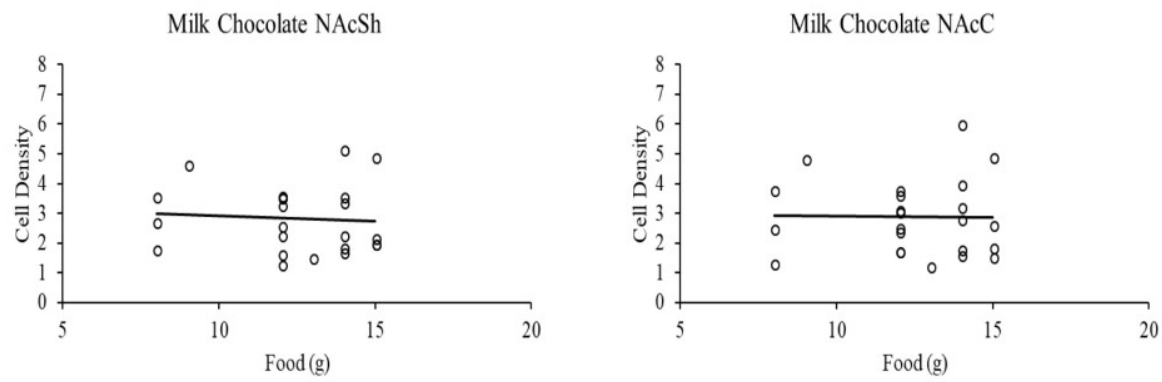

Figure 3.6. Correlations between food ingestion on the day before testing and c-Fos labeling in the NAcSh and NAcC. (A-B) Control group. (C-D) Chocolate pellet group. (E-F) Milk chocolate group. 
(A)

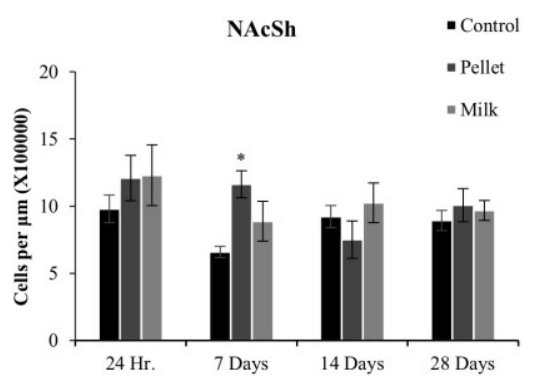

(B)

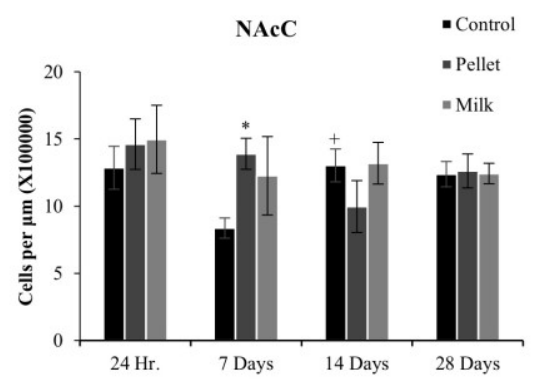

(C)

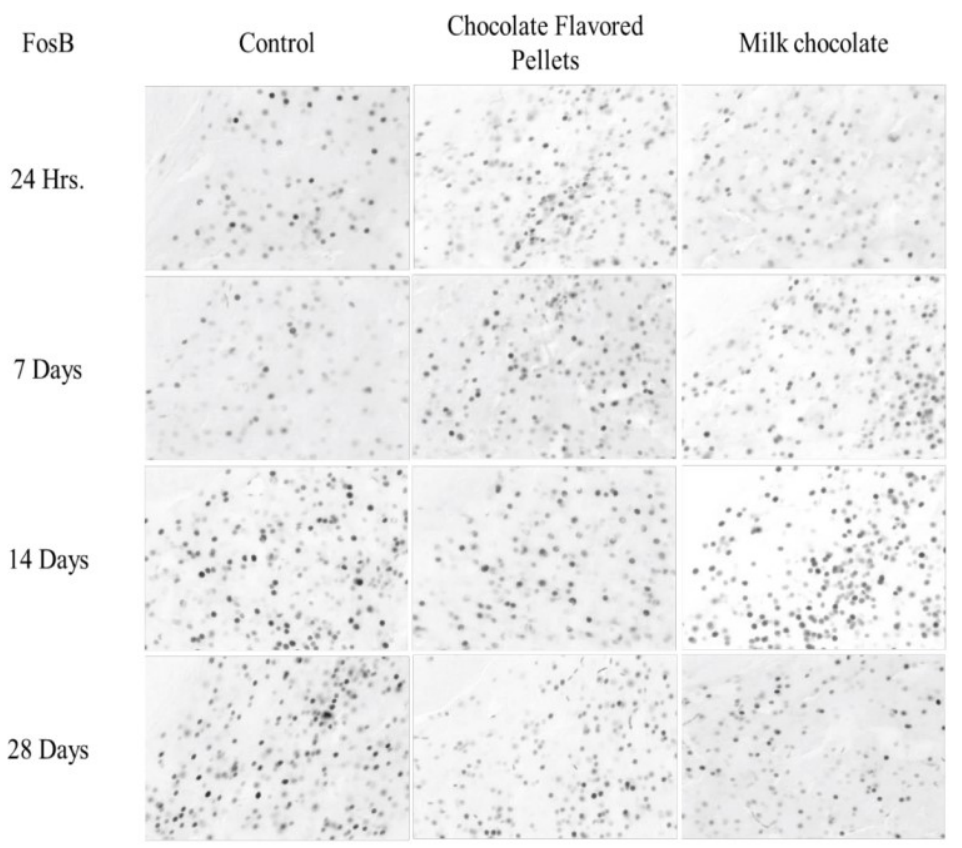

Figure 3.7. Average cell density (mean cell count/volume $\left[\mathrm{mm}^{3}\right]$ ) of FosB labeled neurons (mean $\pm \mathrm{SEM}$ ) (A) NAcSh labeled neurons in rats that underwent CPP for food reward compared to control. ${ }^{*} \mathrm{p}<0.05$ compared to control and milk chocolate at same abstinence period. (B) NAcC labeled neurons in rats that underwent CPP for food reward compared to control. ${ }^{*} \mathrm{p}<0.05$ compared to control at same abstinence period. ${ }^{+} \mathrm{p}<0.05$ compared to control at 7-day abstinence period. (C) Representative images of FosB labelling in the NAc. 20x. 
(A)

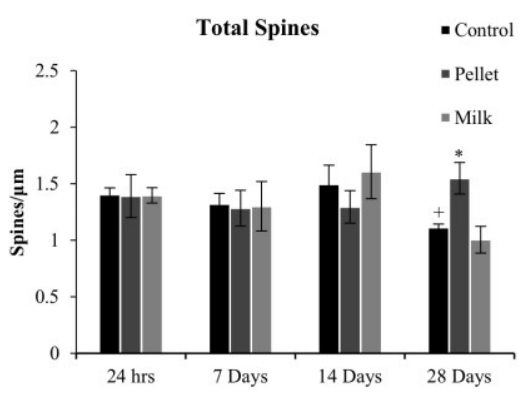

(c)

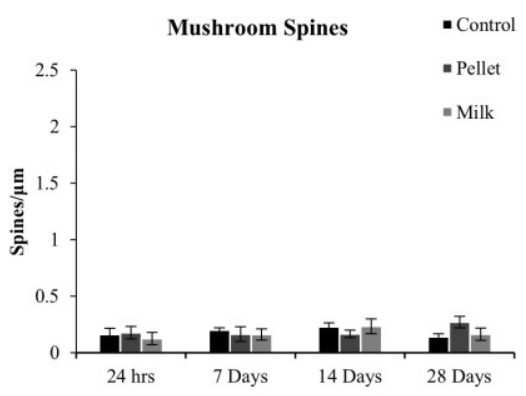

(E)

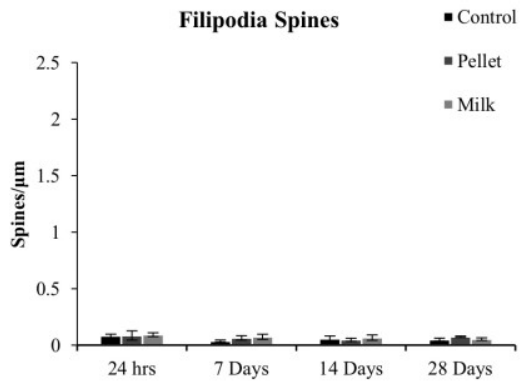

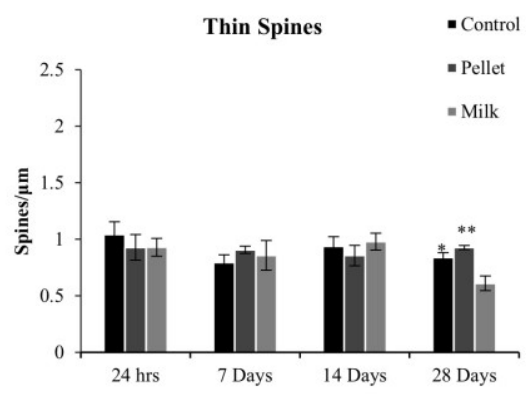

(D)

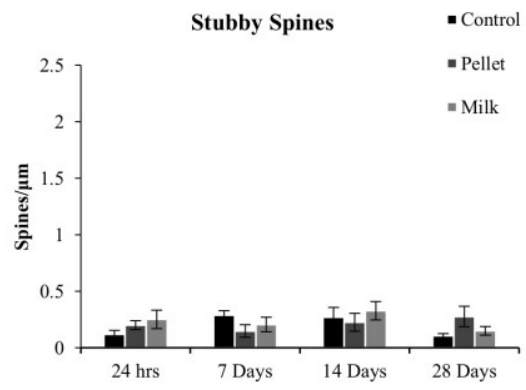

Figure 3.8. Dendritic spine density per $\mu \mathrm{m}$ (Mean \pm SEM) NAcSh. (A) Total Spines. ${ }^{*} \mathrm{p}<0.05$ compared to control and milk chocolate as same abstinence period. $+p<0.05$ compared to control at 14 -day abstinence period (B) Thin Spines. $* \mathrm{p}<0.05$ compared milk chocolate group at same abstinence period. (C) Mushroom spines. (D) Stubby Spines. (E) Filipodia spines. 
(A)

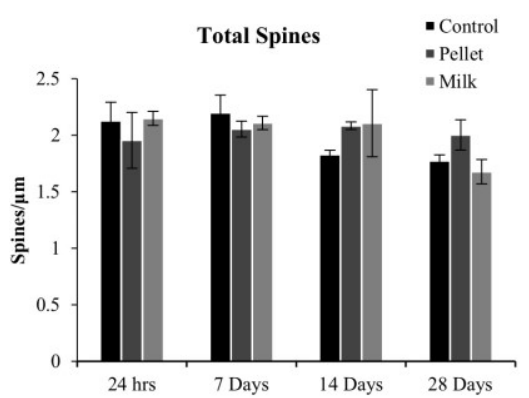

(c)
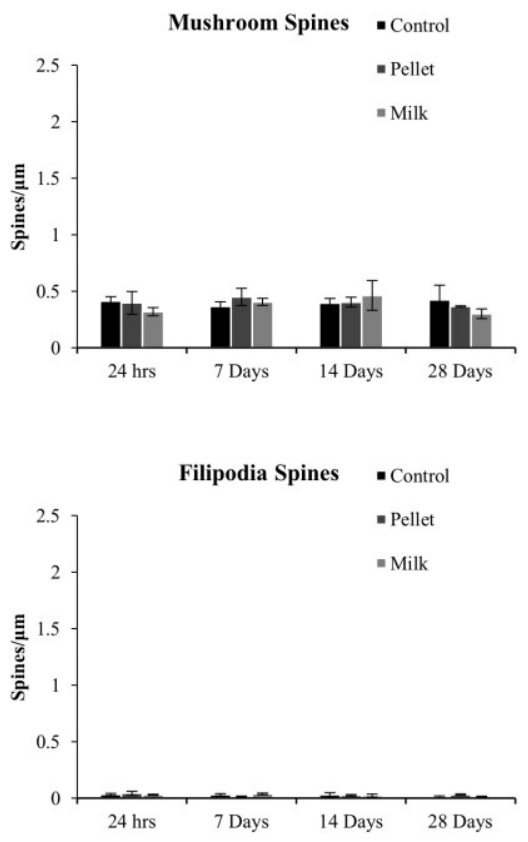

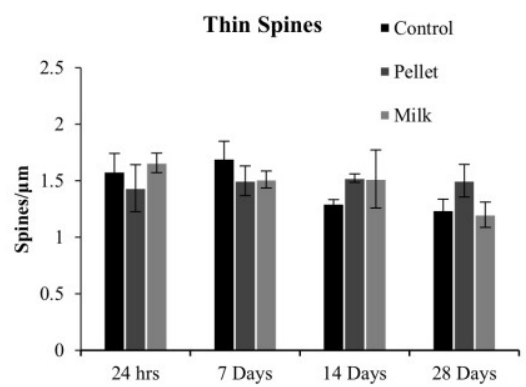

(D)

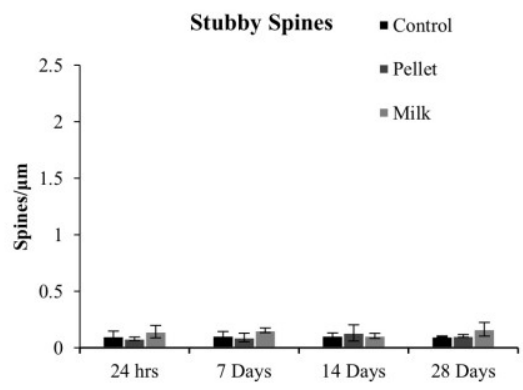

Figure 3.9. Dendritic spine density per $\mu \mathrm{m}$ (Mean \pm SEM) NAcC. (A) Total Spines. (B) Thin Spines. (C) Mushroom spines. (D) Stubby Spines. (E) Filipodia spines. 


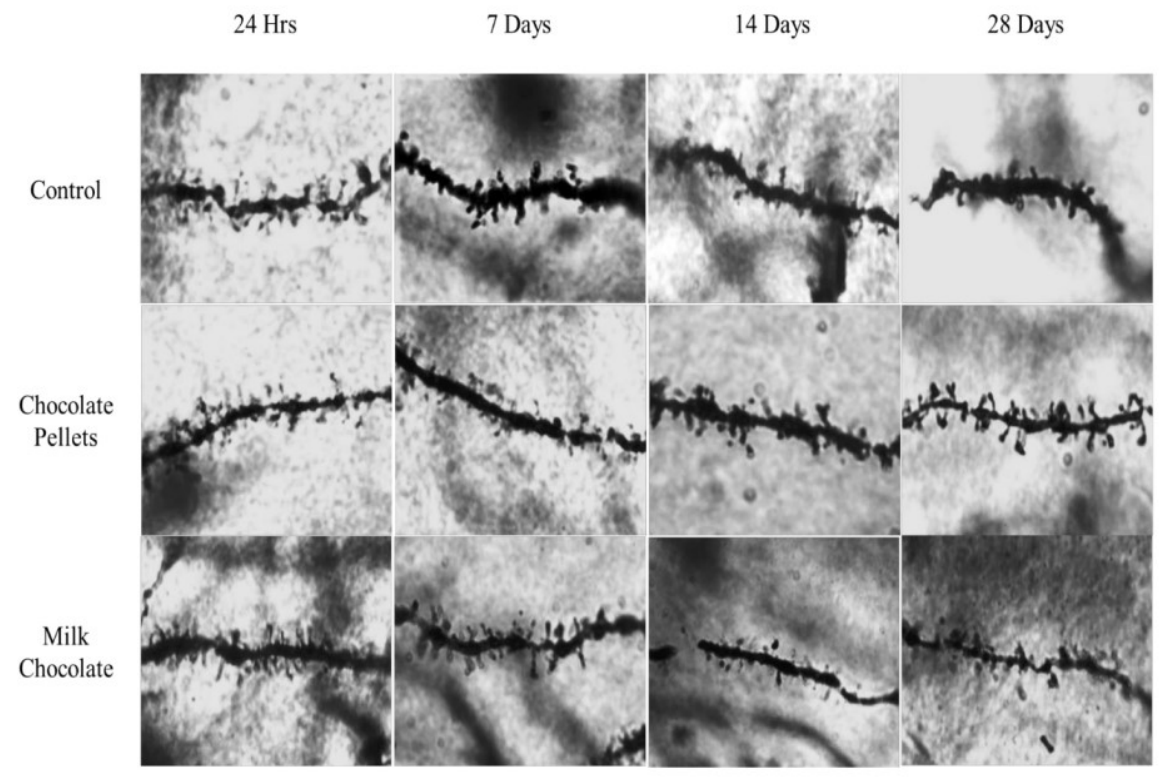

Figure 3.10. Representative images of dendrites analyzed for spine density. 100x. 


\section{Chapter 4}

\section{Time-dependent Changes in GluR1 Protein Expression Following Abstinence From Food Rewards of Different Macronutrient Composition}

Erin W. Noye Tuplin, Madi T. Wright, and Matthew R. Holahan

\subsection{Abstract}

Rationale: Incubation of craving refers to a time-dependent increase in responding for cues associated with reward over extended periods of abstinence. Incubation of cocaine craving is associated with an increase in GluR2-lacking AMPA receptors in the nucleus accumbens (NAc) whereas there is less evidence that this occurs with natural rewards.

Objective: The present study examined whether different macronutrient composition foods and flavors could produce an incubation of craving response and whether this was associated with changes in AMPA receptor protein expression.

Methods: Rats were trained for 10 days to bar press for purified, sucrose, or chocolateflavored sucrose pellets. Food groups underwent forced abstinence for either 24 hrs., 7 days, or 14 days at which point rats underwent an extinction test or were not tested. Following the test, brains from all tested and nontested rats were removed and a 14-gauge punch of the NAc was obtained and rapidly frozen. SDS-Page western blotting procedures were used to determine relative protein expression of GluR1, GluR1 pSer845, and GluR2.

Results: During the test, lever presses, nose pokes into the food hopper, and locomotor activity were recorded and revealed an incubation response in the purified food group. Neither the sucrose nor chocolate group displayed an incubation response. In all groups, GluR1 pSer845 and GluR2 expression did not change across abstinence periods or in response to the reward associated cues. GluR1 expression in the purified and sucrose groups decreased by the 7-day abstinence period and GluR1 expression was higher in tested compared to nontested rats in all food groups. 
Conclusions: Unlike cocaine, we observed a time-dependent decrease in GluR1 protein expression in the NAc, but this effect was not specific to the incubation of craving as it was observed in food groups that did not display an incubation of craving response. This suggests that food reward can induce a change in GluR1 protein expression that declines within a week but may be dependent on food type. Exposure to cues associated with food increased GluR1 expression in all food groups, which may be indicative of stimulus reward learning. The role of GluR1 expression in the incubation of food craving in food restricted rats remains elusive.

\subsection{Introduction}

Following extended periods of abstinence from a rewarding substance, a timedependent enhancement in reward seeking behavior has been noted, termed the "incubation of craving" (Grimm et al., 2005, 2007; Li et al., 2014; Purgianto et al., 2016; Terrier et al., 2015). The study of the incubation of craving has often utilized selfadministration of cocaine (Calu et al., 2007; Grimm et al., 2001, 2002; Halbout et al., 2014; Lu et al., 2004; Thomas et al., 2015), methamphetamine (Li et al., 2015b, 2015a; Shepard et al., 2004), or sucrose (Grimm et al., 2005). Once stable levels of responding occur, rats are tested for cue-induced responding at various periods of abstinence. The incubation of craving is measured in these studies as an increase in responding following longer periods of abstinence from the rewarding substance (i.e. 30 days compared to 24 hrs.).

The type of rewarding substance, and quantity of that substance, may affect the strength of the incubation of craving response and the magnitude of that effect. When comparing morphine and cocaine to food reward, the magnitude of contextual memory for the drugs was greater than it was for food (Baunez et al., 2005; Duarte et al., 2003; Tzschentke, 2007). Specific types of food reward, such as high fat food and regular chow, have both been shown to result in an incubation of craving response following 30 days of abstinence but the magnitude of responding was higher for the high fat food (Dingess et al., 2017b). Comparing high fat and low fat food, a lower percentage of dietary fat intake has been shown to result in increased responding for high fat food rather than the low fat alternative (Tellez et al., 2013).

When rats are given the opportunity to ingest palatable foods, they will ingest more of it, and/or choose it over regular chow (Feltmann et al., 2018; Velázquez-Sánchez et al., 2014a). When food consumption is compared between chocolate flavored sucrose pellets and chow, rats have been shown to consume more of the chocolate sucrose pellets compared to chow highlighting the rewarding properties of this food (Feltmann et al., 2018). In a binge eating model, consumption of chocolate flavored, high sucrose diet resulted in increased consumption in an FR3 schedule operant task in both low and high impulsive animals (Velázquez-Sánchez et al., 2014a). Exposure to high fat foods has also been shown to increase motivation to respond for sucrose reward (De Jong et al., 2012). This highlights that the rewarding properties of sucrose, and the motivation to consume sucrose, is higher than regular chow. 
The nucleus accumbens (NAc) is implicated in mediating the rewarding aspects of substances and underlies reward-seeking behavior (Adinoff, 2004; Chaudhri et al., 2010; Chiara and Di Chiara, 2002; Grimm et al., 2011a; Nestler, 2012; Quintero, 2013). Following abstinence from drugs or sucrose, an increase in AMPA receptors has been observed in the NAc (Conrad et al., 2008; Loweth et al., 2014; Wolf and Tseng, 2012). Synaptic changes associated with long-term abstinence from reward include postsynaptic membrane insertion of GluR2-lacking AMPA receptors, which are composed primarily of GluR1 subunits and are calcium permeable (CP-AMPA) (Malinow and Malenka, 2002). Following abstinence from drugs of abuse, such as cocaine and methamphetamine, CP-AMPA receptors have been shown to be elevated (Scheyer et al., 2016; Wolf and Tseng, 2012). Similarly, following abstinence from natural rewards such as "junk-food" (combination of chips, cookies, lab diet 5001, chocolate flavor, peanut butter) the density of CP-AMPA receptors increased over time in obesity prone rats (Oginsky et al., 2016).

The present study investigated the incubation of craving for food reward and associated changes in AMPA receptor expression. Previous studies from our laboratory have used an FR2 schedule of reinforcement for chocolate pellets which results in robust responding that peaked by day 5 of acquisition (Davis-MacNevin et al., 2013; Holahan et al., 2010, 2012; Tuplin et al., 2015). Since our model utilizes food restriction as a motivation for reward intake, it raises the question as to whether food-seeking is the result of homeostatic or hedonic mechanisms. Drug use and non-homeostatic eating behavior have been compared, as non-homeostatic behaviors are often excessive and involve over consumption of food or drugs (Corwin and Hajnal, 2005). There remains a possibility that food restriction results in a caloric deficit where food seeking behavior may be independent from food palatability (Beeler et al., 2012).

In the present study, the ability of foods with different flavor and macronutrient content to produce an incubation of craving response was explored. In this case, we compared nutritionally purified food pellets, sucrose pellets, and chocolate- flavored sucrose pellets. The goal was to compare sucrose, a highly rewarding food, to chocolate flavor which we have previously shown to not result in an incubation of craving response, to determine if flavor alone affects craving (Noye Tuplin et al., 2018). The study also evaluated how abstinence from each of the pellets affected AMPA receptor subunit expression in the NAc. The expression of GluR1, pGluR1 845, and GluR2 were evaluated, as alterations in the expression of these subunits has been shown following abstinence from rewarding substances, such as cocaine ((Wolf and Tseng, 2012; Zheng et al., 2015)).

\subsection{Methods}

\subsubsection{Animals}

A total of 106 male, Long Evans rats (250-275 grams) from Charles River Quebec was used. Rats were housed individually in clear plastic cages $(25 \times 20 \times 45 \mathrm{~cm})$, given water ad libitum under a 12-hr light-dark cycle (lights on at 8:00 a.m.). Food restriction and handling (5-min daily) began 10 days prior to operant acquisition. Food was

restricted throughout the study to maintain a weight of $90 \%$ baseline. Principles of 
laboratory animal care were followed and all procedures were conducted in accordance with the Canadian Council on Animal Care. The protocol was approved by the Carleton University Animal Care Committee.

\subsubsection{Operant Acquisition}

A total of 90 rats underwent operant acquisition training over a 10-day period during the light phase. Three types of food pellets were utilized in this study and consisted of a pellet that was nutritionally complete (purified food pellet), a sucrose pellet, and a chocolate flavored sucrose pellet (Table 1). Rats were assigned to a pellet type group ( $\mathrm{n}=30$ per food group) and matched based on body weight as measured on the day prior to the start of training. Training consisted of rats being individually placed into an operant chamber (Coulburn Instruments, Whitehall, PA; $30.5 \mathrm{~W}$ x $25.5 \mathrm{D}$ x 30.5 $\mathrm{H} \mathrm{cm}$ ) for $1 \mathrm{hr}$ per day. Every second response (FR2 schedule) on the correct lever resulted in the delivery of a $45 \mathrm{mg}$ food pellet (BioServe, Flemington, NJ). Once the correct lever had been depressed twice, the house light extinguished and the panel lights above the lever changed from red to green for 5 seconds. This was accompanied by the pellet dispenser releasing one 45-mg food pellet into the hopper. The incorrect lever had no programmed consequences. Both correct and incorrect presses, nose pokes into the food hopper, and locomotor activity were recorded during the sessions. Nose pokes into the food hopper were assessed with a photocell beam-break detector that contained an infrared LED source and photodetector on the sides of the food hopper. Locomotor activity was assessed with a ceiling mounted activity monitor tuned to be sensitive to the infrared signals emitted by a rat. Movement units (brief pulses representing the minimum resolution of detection) were used to provide a measure of locomotor activity.

\begin{tabular}{|c|c|c|c|}
\hline$\%$ & Purified & Sucrose & Chocolate \\
\hline Protein & 18.7 & 0 & 0.2 \\
\hline Fat & 5.6 & 0 & 0.1 \\
\hline Carbohydrate & 59.1 & 97.8 & 97.3 \\
\hline
\end{tabular}

Table 4.1. Nutritional profile of purified, sucrose, and chocolate flavored sucrose food pellets.

\subsubsection{Extinction Responding}

Following acquisition, 18 rats from each food group were assigned to one of three abstinence periods and tested at different time delays in the absence of food reward. Rats were matched based on total correct presses on day 10 of acquisition. Rats were assigned to the following periods of abstinence from food reward: 24 hrs., 7 days, or 14 days. The testing session was $1 \mathrm{hr}$. and bar pressing did not result in food reward, but the discrete cues were presented (the house light extinguished, and the panel lights changed from red to green for 5 seconds). Correct presses, incorrect presses, nose pokes into the food hopper, and locomotor activity were recorded at 10-minute intervals. Twelve other rats from each food group were assigned to one of the three abstinence periods but did not undergo testing. 


\subsubsection{Controls}

A fourth group was included that did not undergo operant acquisition. This group was continually food restricted throughout the acquisition and abstinence periods so that total food restriction was equal to the rats that underwent operant acquisition and extinction. Control rats were euthanized at specific abstinence periods ( $24 \mathrm{hr} .: \mathrm{n}=4,7$ days: $\mathrm{n}=3,14$ days: $\mathrm{n}=3$ ).

\subsubsection{Western Blot Analysis}

A minimum of three rats per food type per abstinence period were included in the Western blot analysis. The rats that underwent testing and those that did not, as well as the control rats, were euthanized at approximately the same time of day. Sixty minutes following testing, rats were anesthetized with $1.25 \mathrm{~g} / \mathrm{kg}$ urethane and rapidly decapitated. Brains were removed and placed on ice then sliced using a $1 \mathrm{~mm}$ brain matrix. Using a needle, a 14-gauge, $1 \mathrm{~mm}$ punch was taken from the nucleus accumbens (NAc) (1.2$3.2 \mathrm{~mm}$ from Bregma) and the sample was immediately frozen on dry ice.

\section{Protein Extraction and Quantification}

Tissue was sonicated in pulses in a ripa-like extraction buffer $(100 \mu 1)$ consisting of $0.1 \%$ SDS and $1 \mathrm{mM} \mathrm{Na}$ ortho-vandate in $10 \mathrm{mM}$ tris and EDTA-free protease inhibitor cocktail (Roche). Following sonication, samples were centrifuged at $6000 \mathrm{rpm}$ (4 degrees Celsius) for 10 minutes then placed in new centrifuge tubes while avoiding the protein pellet. Four $\mu \mathrm{l}$ from each sample was loaded into centrifuge tubes containing 56 $\mu 1$ of sterile $0.9 \%$ saline for the end result of a concentration of $1: 15$ of sample. A BCA Protein Assay Kit was used for protein quantification and $25 \mu 1$ of each sample was loaded in duplicate into a 96 well microplate. Two hundred $\mu$ ls of the BSA kit working solution were loaded into each well and the samples were incubated for 30 minutes at 37 degrees Celsius. The samples were then allowed to cool for 10 minutes while remaining covered. The samples were read at $540 \mathrm{~nm}$ and results were multiplied by a dilution factor of 0.8 for a working concentration. Next, $25 \mu \mathrm{l}$ of $5 \mathrm{x}$ loading buffer was added to each sample, a heat cap was added, and samples were heated for 5 minutes at 105 degrees Celsius. Samples were then cooled and stored at -20 for future use.

\section{Western Blot Procedure}

Protein samples were loaded $(20 \mu \mathrm{g})$ into $7.5 \%$ SDS-page gels and electrophoresed at 140 volts for approximately $1 \mathrm{hr}$. using a Powerpac Basic Mini (BioRad). The first lane of the gel was loaded with $5 \mu 1$ of Bio-Rad's Precision Plus Protein Standard (cat \# 161-0374). Membranes containing samples from the same food groups were run simultaneously with the same buffer solution stocks. While the gel was running, membranes (PVDF) were soaked for approximately 1 minute in methanol then transferred to transfer buffer (20\% methanol, tris base, glycine). Four pieces of filter paper (3MM CHr chromatography paper) were soaked in $1 \mathrm{X}$ transfer buffer for approximately 5 minutes before use. Additionally, two sponges were soaked in $1 \mathrm{X}$ transfer buffer. When the dye reached the bottom of the gel the gel was removed and 
floated in $1 \mathrm{X}$ transfer buffer. For transfer from the gel to the membrane, the chamber was filled with pre-chilled $1 \mathrm{X}$ transfer buffer and an ice pack and stir bar were added. A transfer sponge was placed on a transfer cassette and two pieces of filter paper were placed under the gel. The gel was picked up with the filter paper and placed on the transfer sponge. The membrane was then placed on top of the gel and two more pieces of filter paper were added. Another transfer sponge was placed on top of the filter paper to complete the "sandwich". The transfer cassette was then closed and placed into the transfer unit, which was set to a constant voltage of 100 for 60 minutes while stirring at $80 \mathrm{rpm}$. Following the 60 -minute transfer, the membrane was removed from the cassette and allowed to dry for at least 30 minutes protein side up.

Once the membrane was dry, it was rewet with methanol for approximately 30 seconds then rinsed with $\mathrm{DH}_{2} \mathrm{O}$. The membrane was then stained for total protein using $0.0005 \%$ Fast Green for 5 minutes then rinsed with a wash solution $(6.7 \%$ acetic acid in $30 \%$ methanol). Membranes were imaged for 2 minutes on the 700 -channel using a Licor Odyssey Fc then underwent two, 2-minute, washes in TBS. All incubations and washes took place under gentle agitation using a gyrotwister. Membranes were blocked for $1 \mathrm{hr}$. in $0.5 \%$ fish gelatin in TBS. Following the blocking step, the primary antibody (GluR1 1:1000 anti-rabbit Millipore AB1504; GluR2 1:4000 anti-rabbit Millipore AB1768; pGluR1 845 anti-rabbit Millipore) diluted in $0.05 \%$ fish gelatin in TBST was added to the membrane and incubated overnight.

The following day, membranes underwent four 5-minute washes in TBST followed by incubation in the secondary antibody (1:5000 Invitrogen) prepared in $0.5 \%$ fish gelatin with $0.2 \%$ tween and $0.01 \%$ SDS for 1 hour. From this point on, membranes were protected from light. Membranes then underwent four 5-minute washes in TBST, followed by two more 5-minute washes in TBS. Membranes were then placed in fresh TBS and read on the Licor Odyssey Fc system at $700 \mathrm{~nm}$ for 6 minutes.

Total protein expression from each sample on each membrane was determined using the total protein stain method in order to provide normalization across each specific membrane. To determine protein expression, bands at the appropriate molecular weight were determined with the Odyssey software.

\subsubsection{Statistical Analysis}

\section{Operant acquisition}

Correct presses, nose pokes into the food hopper, and locomotor activity during operant acquisition were analyzed with repeated measures ANOVAs using day as the within factor and food group as the between factor. Significant interactions were followed up with one-way ANOVAs between food groups for each day of acquisition. Significant ANOVAs were followed up with Tukey's post hoc comparisons.

\section{Extinction}

To determine if a specific food group displayed an incubation of craving effect, within food group differences across abstinence periods were assessed using one-way ANOVAs. Correct presses, nose pokes into the food hopper, and locomotor activity were 
analyzed separately. Significant ANOVAs were followed up with Tukey's post-hoc comparisons.

\section{Western Blots}

To determine if there was an affect of abstinence period in the untrained control group, one-way ANOVAs with abstinence period as the independent variable and protein (Glur1, GluR1 pSer845, and GluR2) as the independent variable were performed. Significant ANOVAs were followed up with Tukey's post-hoc comparisons.

For each food group, western blots were calculated as relative expression from control and each food group was analyzed separately. Fixed factor, univariate ANOVAs with abstinence period ( 24 hrs., 7 days, 14 days) and testing condition (test vs no test) and factors were performed for each AMPA receptor protein. Significant ANOVAs were followed up with Tukey's post-hoc comparisons.

\subsection{Results}

\subsubsection{Operant Acquisition}

A repeated measures ANOVA on correct presses with day as the repeated factor and food group at the between factor revealed a main effect of day $(F(9,774)=109.152, p$ $<0.001)$, food group $(\mathrm{F}(2,86)=24.202, \mathrm{p}<0.001)$, and a significant interaction $(\mathrm{F}(18,774)=4.335, \mathrm{p}<0.001 ; \mathrm{Fig} 1 \mathrm{~A})$. Tukey's post-hoc analysis on the group effect revealed that the purified food pellet group emitted significantly more correct presses than both the sucrose and chocolate groups $(\mathrm{p}<0.001)$. There were no significant differences in correct presses between the sucrose and chocolate groups $(p>0.05)$. Oneway ANOVAs on each day of acquisition revealed main effects of food group on all days except day 2. Tukey's post-hoc analyses revealed that the purified group produced significantly more correct presses on days 3 to 10 of acquisition compared to the sucrose and chocolate groups $(\mathrm{p}<0.01)$. On day 1 , the purified group showed significantly more correct presses than the chocolate group $(\mathrm{p}<0.05)$. The sucrose and chocolate groups did not significantly differ from each other on any day $(\mathrm{p}>0.05)$.

A repeated measures ANOVA on nose pokes into the food hopper using day as the repeated factor and food group as the between factor revealed a main effect of day $(\mathrm{F}(9,774)=20.071, \mathrm{p}<0.001)$ and food group $(\mathrm{F}(2,86)=170718, \mathrm{p}<0.001)$, but no interaction $(\mathrm{F}(18,774)=0.662, \mathrm{p}>0.05 ;$ Fig $1 \mathrm{~B})$. Tukey's post-hoc analysis on the main effect of day revealed that the purified food pellet group made significantly more nose pokes into the food hopper than the sucrose and chocolate groups $(p<0.05)$. The sucrose group made significantly more nose pokes than the chocolate group $(\mathrm{p}<0.01)$.

A repeated measures ANOVA on locomotor activity using day as the repeated factor and food group as the between factor revealed a main effect of day $(\mathrm{F}(9,774)=$ $7.538, \mathrm{p}<0.001)$ but no main effect of food group $(\mathrm{F}(2,86)=2.718, \mathrm{p}=0.072)$ and no interaction $(\mathrm{F}(18,774)=0.526$; Fig $1 \mathrm{C})$. 


\subsubsection{Extinction Responding \\ Correct Presses}

A one-way ANOVA on correct presses within the purified food group with abstinence period as the independent variable revealed a main effect of abstinence period $(\mathrm{F}(2,14)=6.932, \mathrm{p}<0.01 ; \mathrm{Fig} 2 \mathrm{~A})$. Tukey's post-hoc analysis revealed significantly more correct presses on 14-day abstinence period than the 24-hr. period $(\mathrm{p}<0.01)$. In the sucrose group, a one-way ANOVA on correct presses with abstinence period as the independent variable did not reveal a main effect of abstinence period $(\mathrm{F}(2,18)=1.220, \mathrm{p}$ $>0.05$; Fig 2B). In the chocolate group, a one-way ANOVA on correct presses with abstinence period as the independent variable did not reveal a main effect of abstinence period $(\mathrm{F}(2,18)=0.036$; Fig $2 \mathrm{C})$.

\section{Nose Pokes}

A one-way ANOVA on nose pokes into the food hopper within the purified food group with abstinence period as the independent variable did not reveal a main effect of abstinence period $(F(2,14)=1.571, p>0.05$; Figure $4.2 D)$. In the sucrose group, a oneway ANOVA on nose pokes into the food hopper with abstinence period as the independent variable did not reveal a main effect of abstinence period $(\mathrm{F}(2,18)=3.015, \mathrm{p}$ $>0.05$; Fig 2E). In the chocolate group, a one-way ANOVA on nose pokes into the food hopper with abstinence period as the independent variable did not reveal a main effect of abstinence period $(\mathrm{F}(2,18)=2.529, \mathrm{p}>0.05$; Fig $2 \mathrm{~F})$.

\section{Locomotor Activity}

A one-way ANOVA on locomotor activity within the purified food group with abstinence period as the independent variable revealed a main effect of abstinence period $(\mathrm{F}(2,14)=5.661, \mathrm{p}<0.05 ; \mathrm{Fig} 2 \mathrm{G})$. Tukey's post-hoc analysis revealed significantly higher levels of locomotor activity on the 14-day abstinence period than $24 \mathrm{hrs}$. (p < 0.05 ). In the sucrose group, a one-way ANOVA on locomotor activity with abstinence period as the independent variable did not reveal a main effect of abstinence period $(\mathrm{F}(2,18)=0.032 ; \mathrm{Fig} 2 \mathrm{H}$. In the chocolate group, a one-way ANOVA on locomotor activity with abstinence period as the independent variable did not reveal a main effect of abstinence period $(\mathrm{F}(2,18)=1.008$; Fig 2I).

\subsubsection{GluR2 Protein Expression}

Representative images of GluR2 stained bands are shown in Figure 4.3A. To determine the effect of abstinence period on GluR2 expression within the food-restricted control group a one-way ANOVA with abstinence period as the independent variable and GluR2 expression as the dependent variable was performed. This revealed no main effect of abstinence period in the control group $(\mathrm{F}(2,7)=0.517, \mathrm{p}>0.05)$. 


\section{Purified Food Pellets}

A fixed factor, univariate ANOVA with abstinence period ( $24 \mathrm{hr}, 7 \mathrm{~d}$, and $14 \mathrm{~d}$ ) and testing conditioning (test or no test) as the factors comparing relative GluR2 expression (relative to control levels) revealed no main effect of testing condition $(\mathrm{F}(1,18)=0.446, \mathrm{p}>0.05)$ or abstinence period $(\mathrm{F}(2,18)=0.583, \mathrm{p}>0.05)$ and no interaction $(\mathrm{F}(2,18)=0.858, \mathrm{p}>0.05)$ in the purified food pellet group (Fig 3B).

\section{Sucrose Pellets}

A fixed factor, univariate ANOVA with abstinence period ( $24 \mathrm{hr}, 7 \mathrm{~d}$, and $14 \mathrm{~d}$ ) and testing conditioning (test or no test) as the factors comparing relative GluR2 expression (relative to control levels) revealed a main effect of abstinence period $(\mathrm{F}(2,18)$ $=3.956, \mathrm{p}<0.05)$ but no effect of test condition $(\mathrm{F}(1,18)=0.466, \mathrm{p}>0.05)$ and no interaction, $(\mathrm{F}(2,18)=0.619, \mathrm{p}>0.05)$ in the sucrose pellet group (Fig $3 \mathrm{C})$.

\section{Chocolate Pellets}

A fixed factor, univariate ANOVA with abstinence period ( $24 \mathrm{hr}, 7 \mathrm{~d}$, and $14 \mathrm{~d})$ and testing conditioning (test or no test) as the factors comparing relative GluR2 expression (relative to control levels) revealed no main effect of testing condition $(\mathrm{F}(1,17)=1.304, \mathrm{p}>0.05)$, no effect of abstinence period $(\mathrm{F}(2,17)=1.323, \mathrm{p}>0.05)$ and no interaction, $(\mathrm{F}(2,17)=0.164, \mathrm{p}>0.05)$ in the chocolate pellet group (Fig 3D).

\subsubsection{GluR1 Protein Expression}

Representative images of GluR1 stained bands are shown in Figure 4.4A. To determine the effect of abstinence period on GluR1 expression within the food-restricted control group, a one-way ANOVA with abstinence period as the independent variable and GluR1 expression as the dependent variable was performed. This revealed a significant main effect of abstinence period within the control group $(F(2,7)=8.174, p<$ $0.05)$. Tukey post-hoc analysis on abstinence period within the control group revealed significantly lower GluR1 expression at the 14-day abstinence period compared to $24 \mathrm{hrs}$. and 7 days $(\mathrm{p}<0.05)$.

\section{Purified Food Pellets}

A fixed factor, univariate ANOVA with abstinence period (24 hr, $7 \mathrm{~d}$, and $14 \mathrm{~d}$ ) and testing conditioning (test or no test) as the factors comparing relative GluR2 expression (relative to control levels) revealed a main effect of testing condition $(\mathrm{F}(1,13)$ $=12.447, \mathrm{p}<0.01)$ and abstinence period $(\mathrm{F}(2,13)=7.966, \mathrm{p}<0.01)$ but no interaction $(\mathrm{F}(2,13)=2.523, \mathrm{p}>0.05)$ in the purified food pellet group (Fig 4B). Tukey's post-hoc analysis on abstinence period revealed that at the 7-day period, rats had significantly lower GluR1 expression compared to the 24-hr. and 14-day periods. 


\section{Sucrose Pellets}

A fixed factor, univariate ANOVA with abstinence period ( $24 \mathrm{hr}, 7 \mathrm{~d}$, and $14 \mathrm{~d}$ ) and testing conditioning (test or no test) as the factors comparing relative GluR2 expression (relative to control levels) revealed a main effect of testing condition $(\mathrm{F}(1,18)$ $=5.945, \mathrm{p}<0.05)$ and abstinence period $(\mathrm{F}(2,18)=5.714, \mathrm{p}<0.05)$ but no interaction $(\mathrm{F}(2,18)=0.088, \mathrm{p}>0.05)$ in the sucrose pellet group (Fig 4C). Tukey's post-hoc analysis on abstinence period revealed that at the 7-day period rats had significantly lower GluR1 expression relative to controls compared to the 24-hr. and 14-day periods.

\section{Chocolate Pellets}

A fixed factor, univariate ANOVA with abstinence period ( $24 \mathrm{hr}, 7 \mathrm{~d}$, and $14 \mathrm{~d}$ ) and testing conditioning (test or no test) as the factors comparing relative GluR2 expression (relative to control levels) revealed a main effect of testing condition $(\mathrm{F}(1,12)$ $=10.301, \mathrm{p}<0.01)$ but no main effect of abstinence period $(\mathrm{F}(2,12)=1.399, \mathrm{p}>0.05)$ and no interaction $(\mathrm{F}(2,12)=2.835, \mathrm{p}>0.05)$ in the chocolate pellet group (Fig 4D).

\subsubsection{GluR1 pSer845 Expression}

Representative images of GluR1 pSer845 stained bands are shown in Figure 4.5A. To determine the effect of abstinence period on GluR1 pSer845 expression within the food-restricted control group a one-way ANOVA with abstinence period as the independent variable and GluR1 expression as the dependent variable was performed (Fig 8A). There was no significant effect of abstinence period on GluR1 pSer845 expression within the control group $(\mathrm{F}(2,7)=0.564, \mathrm{p}>0.05)$.

\section{Purified Food Pellets}

A fixed factor, univariate ANOVA with abstinence period (24 hr, $7 \mathrm{~d}$, and $14 \mathrm{~d}$ ) and testing conditioning (test or no test) as the factors comparing relative GluR1 pSer845 expression (relative to control levels) revealed no main effect of testing condition $(\mathrm{F}(1,13)=0.753, \mathrm{p}>0.05)$ or abstinence period $(\mathrm{F}(2,13)=2.153, \mathrm{p}>.05)$ and no interaction $(\mathrm{F}(2,13)=3.116, \mathrm{p}=0.078)$ in the purified food pellet group (Fig 5B).

\section{Sucrose Pellets}

A fixed factor, univariate ANOVA with abstinence period ( $24 \mathrm{hr}, 7 \mathrm{~d}$, and $14 \mathrm{~d}$ ) and testing conditioning (test or no test) as the factors comparing relative GluR1 pSer845 expression (relative to control levels) revealed no main effect of testing condition $(\mathrm{F}(1,18)=0.609, \mathrm{p}>0.05)$ or abstinence period $(\mathrm{F}(2,18)=0.295, \mathrm{p}>0.05)$ and no interaction $(\mathrm{F}(2,18)=0.546, \mathrm{p}>0.05)$ in the sucrose pellet group (Fig 5C).

\section{Chocolate Pellets}

A fixed factor, univariate ANOVA with abstinence period (24 hr, $7 \mathrm{~d}$, and $14 \mathrm{~d}$ ) and testing conditioning (test or no test) as the factors comparing relative GluR1 pSer845 
expression (relative to control levels) revealed no main effect of testing condition $(\mathrm{F}(1,18)=0.609, \mathrm{p}>0.05)$ or abstinence period $(\mathrm{F}(2,18)=0.295, \mathrm{p}>0.05)$ and no interaction $(\mathrm{F}(2,18)=0.546, \mathrm{p}>0.05)$ in the chocolate pellet group (Fig 5D).

\subsection{Discussion}

The present study examined whether foods of different macronutrient composition and flavor would result in an incubation of craving response and whether this was associated with changes in AMPA receptor protein expression. Abstinence from self-administration of the natural reinforcer liquid sucrose (Grimm et al., 2005, 2011a) and foods (Darling et al., 2016; Dingess et al., 2017b; Krasnova et al., 2014d) has been shown to result in an incubation of craving response. In the present study, an incubation response was noted in the purified food pellet group where responding was higher at abstinence day 14 compared to 24 hours. The sucrose and chocolate pellet groups did not display an incubation effect but did display consistent responding at all abstinence periods suggesting that associative learning did occur during acquisition.

The quantity of reward during acquisition has been shown to differ depending on the reward choice. For example, different flavors, such as chocolate beverage vs milk beverage, have been shown to differentially affect food intake, where rats ingest more milk beverage compared to the chocolate flavored beverage, highlighting the importance of investigating different flavors of rewarding substances (Loi et al., 2013). One key difference between the purified food pellet group and the sucrose and chocolate groups was the level of responding during acquisition, which affected the amount of food reward consumed. The purified group pressed the correct lever significantly more than the other groups during acquisition, thus ingesting more food. One possibility for this was that rats in the sucrose and chocolate groups became satiated during the $1 \mathrm{hr}$. acquisition period. Other incubation of craving studies involving natural rewards, such as high fat food, involve time-outs as part of the conditioning, which may prevent satiation (Darling et al., 2016; Dingess et al., 2017b; Krasnova et al., 2014c). Animals that reach satiation during training continue to respond to rewarding cues, but will not continue bar pressing during acquisition (Colagiuri and Lovibond, 2015). In the present study, by day 10 of acquisition, rats in the purified group averaged 400 pellets per day while the sucrose and chocolate groups averaged 200. It is possible that satiation, and less reward ingestion, may have affected subsequent craving.

The long-term use of food restriction in the present study differs from others that did not use food restriction or only used it to initiate motivation for reward (Krasnova et al., 2014c). Rats in a food restricted state may seek food in order to restore homeostasis, which involves the restoration of energy balance and avoidance of negative affect (Corwin and Hajnal, 2005). The higher bar pressing during acquisition in the purified group could have been the result of homeostatic mechanisms where rats were trying to reach an energy balance, as these pellets were nutritionally complete. Dopamine signaling is important in the regulation of feeding behavior and maintenance of homeostatic balance (Ferrario et al., 2016). In food restricted rats, an amplified release of DA has been shown in response to food (Ferrario et al., 2016). Alterations in the DA system in food-restricted rats is also evidenced by an amplified response to drugs such as 
cocaine (Carr et al., 2000). It is possible that food restriction enhanced responding for cues and prevented an incubation effect, particularly in the sucrose and chocolate groups. The purified group was also food restricted but overall ingested twice as many food pellets during acquisition training. This resulted in the overall food intake being similar to ad lib fed rats. The decrease in food consumption during the periods of abstinence would have been greater for the purified group and may have been a factor in the increased cue responding at 14 days compared to $24 \mathrm{hrs}$. of abstinence.

Evidence suggests that long-term potentiation (LTP) associated with craving involves the insertion of postsynaptic CP-AMPA receptors, which are composed primarily of GluR1 protein subunits (Malinow and Malenka, 2002). Time-dependent accumulation of CP-AMPA receptors has been shown following abstinence from cocaine (Wolf and Tseng, 2012), methamphetamine (Scheyer et al., 2016), and "junk-food" (Oginsky et al., 2016). In the present study, there was a decrease in GluR1 expression at the 7-day abstinence period in the sucrose and purified groups but not the chocolate group. A study by Counotte and colleagues (2014) investigated time-dependent changes in AMPA/NMDA ratio and incubation of sucrose craving in adolescent and adult rats (Counotte et al., 2014). Following 21 days of abstinence from sucrose, responding for cues was higher compared to $24 \mathrm{hrs}$. which was associated with a decrease in AMPA/NMDA ratio and no change in CP-AMPA receptors (Counotte et al., 2014). The authors suggested that sucrose reward decreases post-synaptic strength and that plasticity mechanisms may differ from those induced by cocaine (Counotte et al., 2014). In the present study, the time-dependent decrease in GluR1 expression was unexpected as the mechanisms of drug and food reward have been shown to overlap (Kelley and Berridge, 2002). GluR2 protein expression did not change in response to rewarding cues or abstinence suggesting there was no increase in GluR2-containing receptors. The GluR2 expression in the present study is similar to what has been seen following drug withdrawal (Loweth et al., 2014) It was also surprising that GluR1 decreased over time in both the purified and sucrose groups where only the purified group showed an incubation response. This suggests that the decrease in GluR1 expression did not play a functional role in the incubation of craving response observed following 14 days of abstinence in the purified group.

The timeline of CP-AMPA receptor accumulation following abstinence from reward self-administration has been shown to differ depending on the type of reward. Following abstinence from cocaine self-administration, the density of CP-AMPA receptors remains elevated following 30 days of abstinence (Wolf and Tseng, 2012). Following abstinence from methamphetamine, CP-AMPA receptors are increased earlier than cocaine, within one week of abstinence (Scheyer et al., 2016). Abstinence from a junk food natural reinforcer resulted in an increased density of CP-AMPA receptors that persisted for 21 days of abstinence in rats susceptible to obesity (Oginsky et al., 2016). These studies highlight the differential affect of each reward type on the accumulation of CP-AMPA receptors in the NAc. In the present study, GluR1 expression was not measured beyond 14 days of abstinence, leading to the possibility that this was too early of a time point to see a significant accumulation. In cocaine studies, modest changes in CP-AMPA receptors have been noted following 21 days of abstinence, but it was not as profound as later time periods (Conrad et al., 2008). 
Exposure to sucrose has been shown to rapidly induce GluR1 at the post-synaptic density in the NAc (Peng et al., 2011b). This has been shown to be present whether rats were tested immediately after sucrose or $24 \mathrm{hrs}$. later (Peng et al., 2011b). In the present study, the higher GluR1 expression at 24 hrs versus 7 days could have been the result of food intake during day 10 of acquisition. Rats that were administered the extinction test also showed elevated GluR1 expression overall compared to rats that underwent acquisition but not testing. This was unsurprising as cues gain incentive value following pairing with rewarding substances. Since GluR1 can be rapidly induced following rewarding food, it is probable that cues previously associated with reward resulted in a similar effect.

Phosphorylation of GluR1 at Ser845 is involved in the stabilization and trafficking of GluR1-containing AMPA receptors at the synapse (Carr, 2016). Ingestion of palatable food has been shown to activate downstream targets of D1 receptor signaling that have been associated with the phosphorylation of GluR1 at Ser845 (Carr, 2016). Food restriction alone has been associated with an increase in Ser845 phosphorylation following D1 DA receptor stimulation compared to controls suggesting a sensitized DA system (Carr et al., 2010). In the present study, no changes in GluR1 pSer845 were noted in any food group or abstinence period, but there were changes in GluR1 expression. Since pSer845 is involved in synaptic insertion of AMPA receptors and there were no significant group differences, it leads to the possibility that the changes in GluR1 expression are the result of intracellular GluR1 proteins rather than synaptically inserted receptors. In the present study, it is also possible that the rewarding affects of these foods did not sufficiently activate the DA system more than food restricted controls blunting the phosphorylation of pSer845 on the GluR1 protein.

\subsection{Conclusions}

The present study has shown that exposure to cues previously associated with food reward can rapidly increase GluR1 protein expression. This rapid increase was not specific to incubation of craving and was present in all food groups. The time-dependent decrease in GluR1 expression in the purified and sucrose groups was also not specific to incubation of craving and highlights that foods of similar macronutrient composition, but different flavors (sucrose vs chocolate flavored sucrose), can differentially affect AMPA activity in the NAc. The phosphorylation of Ser845 on the GluR1 protein did not change over time in any of the groups suggesting that the surface expression of GluR1 may not have changed over time. This study highlights that the functional significance of GluR1 in the incubation of craving for food reward in food restricted rats remains elusive. 


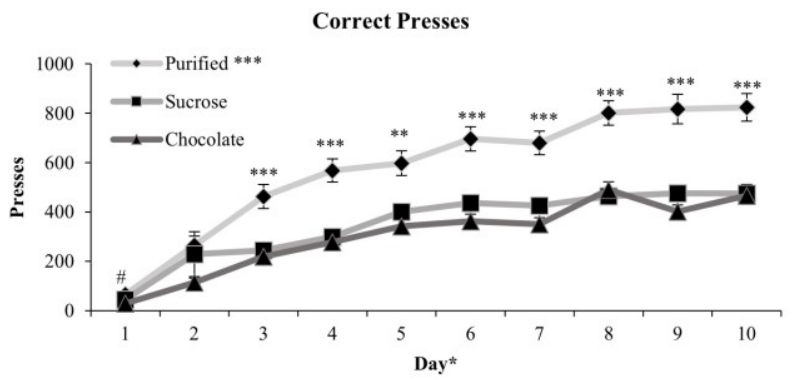

(B)

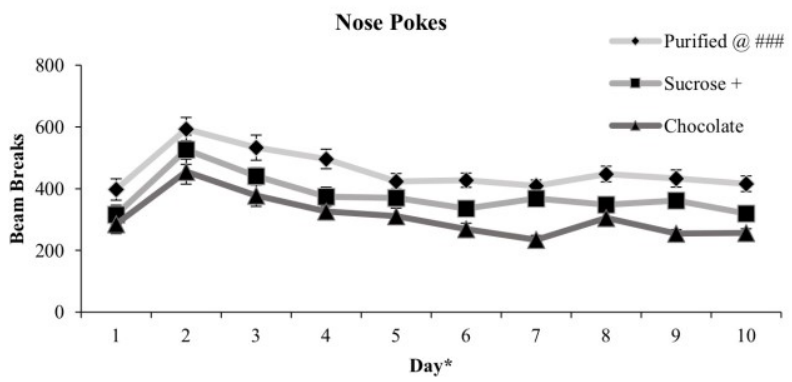

(C)

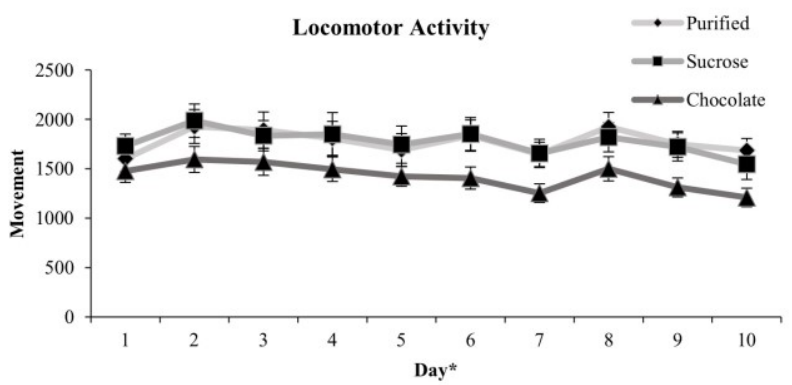

Figure 4.1. Operant Acquisition (Mean \pm SEM). (A) Correct presses. ${ }^{* * *} p<0.001$ compared to sucrose and chocolate groups at same day and overall. ${ }^{*} \mathrm{p}<0.01$ compared to sucrose and chocolate groups at same day. (B) Nose pokes. @ p $<0.05$ compared to sucrose. \#\#\#p $<0.001$ compared to chocolate. $+\mathrm{p}<$ 0.05 compared to chocolate (C) Locomotor Activity 
Extinction Test

Purified

(A)

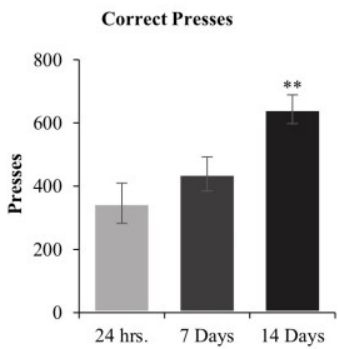

(D)

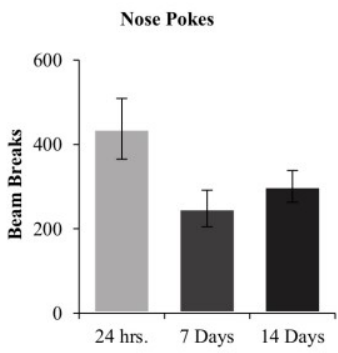

(G)

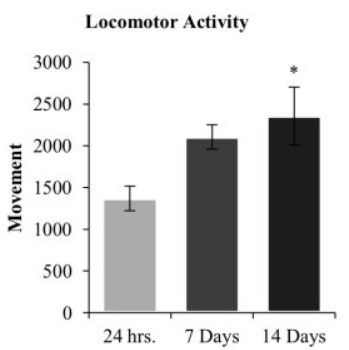

Sucrose

(B)

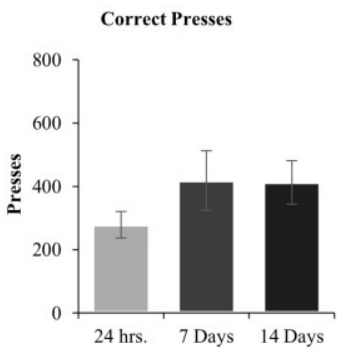

(E)

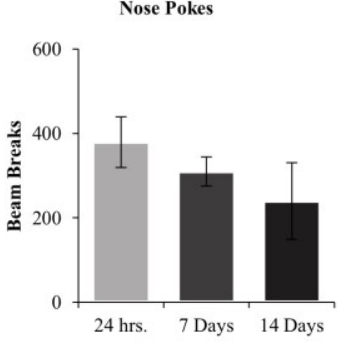

(H)

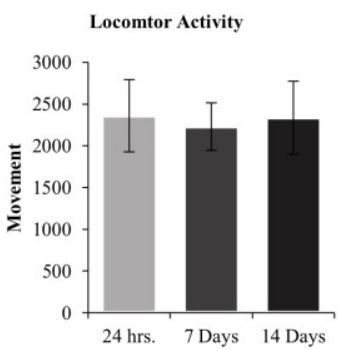

Chocolate

(C)

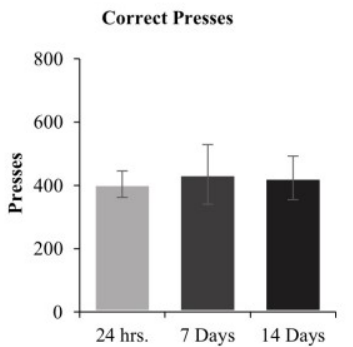

(F)

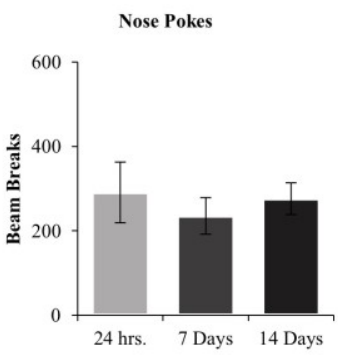

(I)

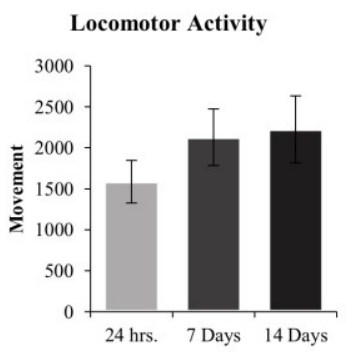

Figure 4.2. Extinction test (Mean \pm SEM). (A) Correct presses; purified group. ${ }^{* *} p<0.05$ more than 24 hrs. (B) Correct presses; sucrose group. (C) Correct presses; chocolate group. (D) Nose pokes; purified group. (E) Nose pokes; sucrose group. (F) Nose Pokes; chocolate group. (G) Locomotor activity; purified group. ${ }^{*} \mathrm{p}<0.05$ more than 24 hrs. (H) Locomotor activity; sucrose group. (I) locomotor activity; chocolate group. 
(A)

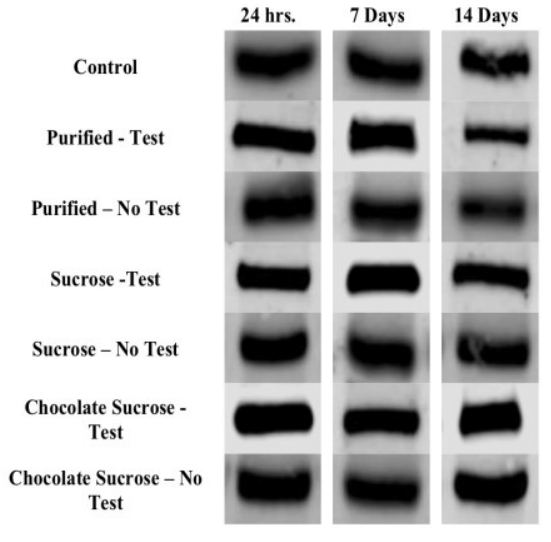

(C)

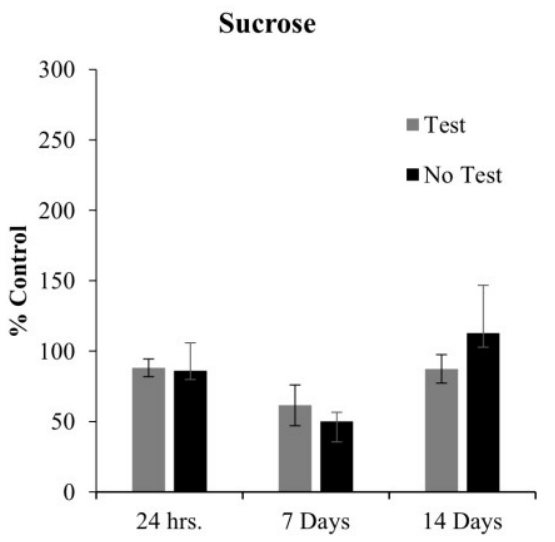

(B)

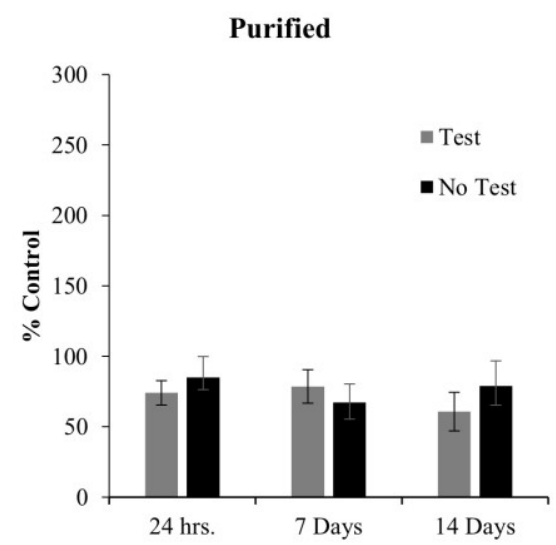

(D)

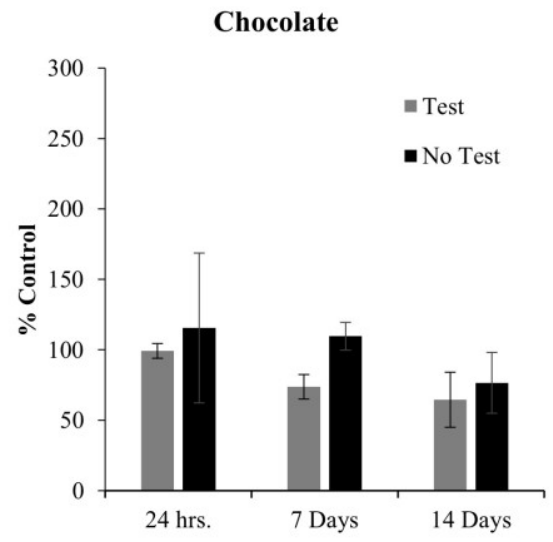

Figure 4.3. GluR2 expression relative to control (Mean $\pm \mathrm{SEM}$ ). (A) representative bands. (B) Purified group. (C) Sucrose group. (D) Chocolate group. 
(A)

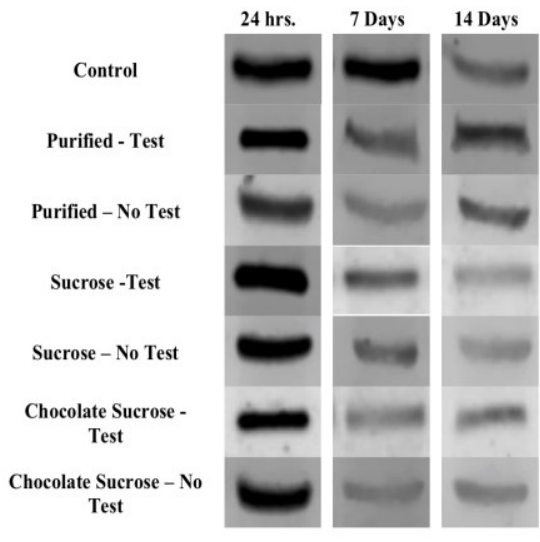

(C)

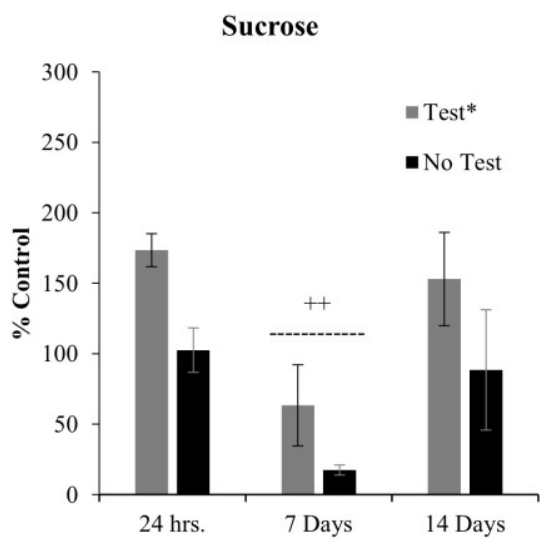

(B)

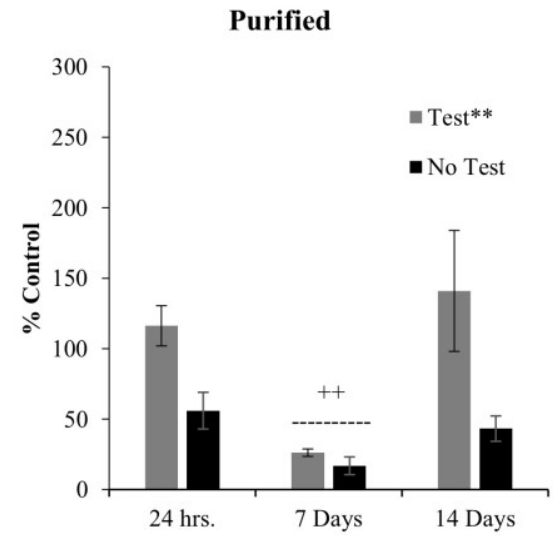

(D)

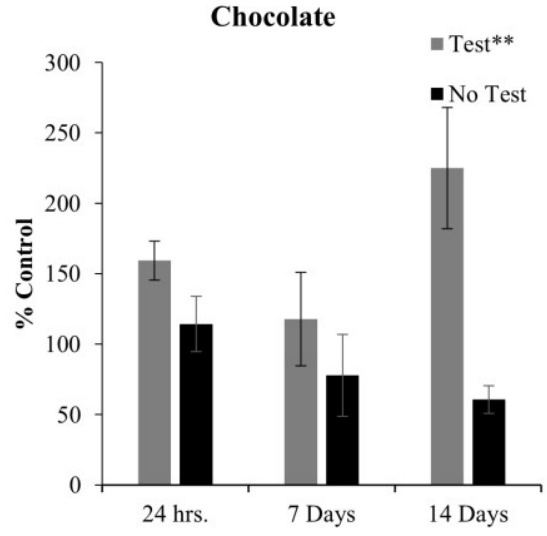

Figure 4.4. Glur1 Expression relative to control (Mean \pm SEM). (A) Representative bands. (B) Purified group. $++\mathrm{p}<0.01$ compared to $24 \mathrm{hrs}$. and 14 days. ${ }^{* *} \mathrm{p}<0.05$ test vs no test. (C) Sucrose group. $++\mathrm{p}<$ 0.01 compared to 24 hrs. and 14 days. ${ }^{*} p<0.05$ test vs no test. (D) Chocolate group. ${ }^{* * p}<0.01$ test vs no test. 
(A)

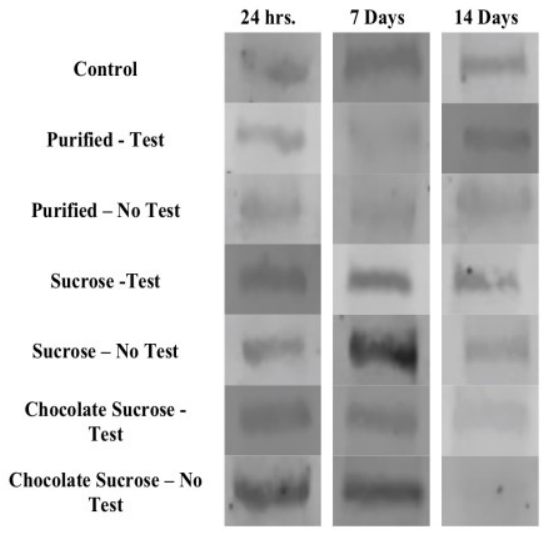

(C)

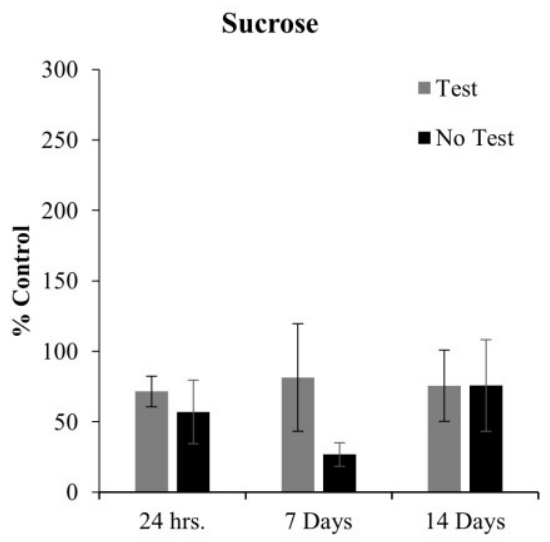

(B)

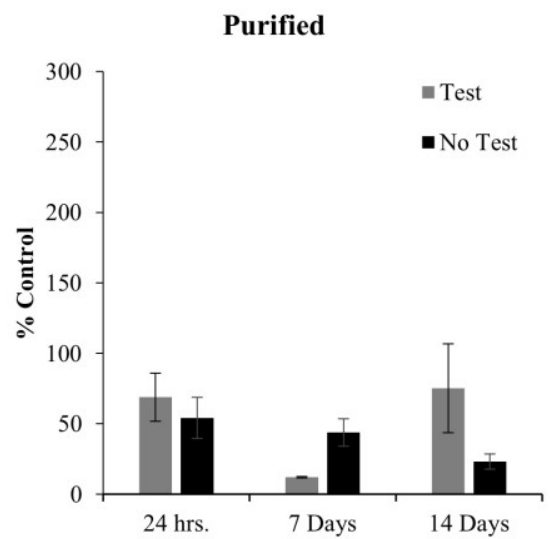

(D)

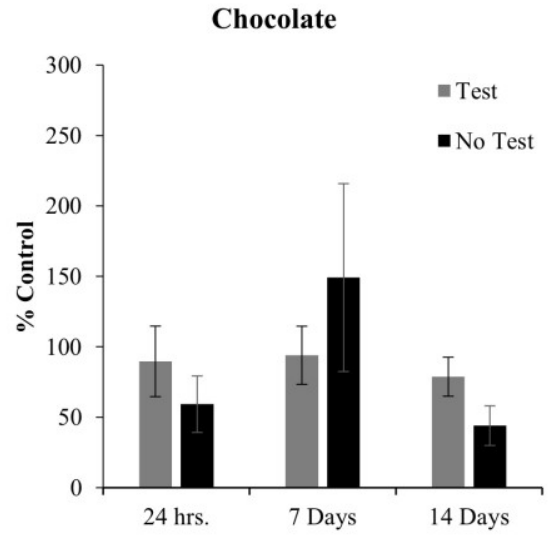

Figure 4.5. GluR1 pSer845 expression relative to control (Mean \pm SEM). (A) Representative bands. (B) Purified group. (C) Sucrose Group. (D) Chocolate group. 


\section{Chapter 5}

\section{General Discussion}

The aim of the present thesis was to investigate the incubation of craving for food reward utilizing both contingent and non-contingent behavioral designs and to investigate associated changes in the NAc. Different food rewards were compared to determine if the ability to produce an incubation effect, and the magnitude of that effect, was dependent on food type. Chapter II explored the effect of operant (contingent) or classical (non-contingent) conditioning for chocolate pellets in responding for cues during periods of force abstinence. Associated changes in c-Fos, FosB, and dendritic spine density in the NAc were explored. Chapter II demonstrated that neither operant nor classical conditioning resulted in an incubation of craving response but responding for cues remained consistent across all abstinence periods which was associated with elevated spine density. It was demonstrated that there were clear differences between operant and classical conditioning in c-Fos and FosB labelling in the NAc. Classical conditioning resulted in less c-Fos and FosB labelling in the NAc compared to the food restricted control and operant conditioned rats. The functional significance of these changes has yet to be elucidated but the study highlighted that contingent and noncontingent food administration differentially activates the NAc. Both the operant and classically conditioned rats displayed elevated spine density at all abstinence periods compared to food restricted controls that did not undergo behavioral procedures. Elevated spine density is associated with increased incentive value for a reward and in this case was associated with behavioral learning. These findings suggest that long-term functional and structural changes are present following at least 28 days of abstinence from chocolate flavored pellets.

Chapter III was a follow-up to chapter II and further explored non-contingent administration of food reward by utilizing a CPP design and explored place preference for foods of different macronutrient compositions. Chocolate flavored pellets and milk chocolate chips were compared for time-dependent changes in place preference following periods of forced abstinence. Similar to chapter II, c-Fos, FosB, and dendritic spine density in the NAc were assessed. Neither chocolate flavored pellet nor milk chocolate chip administration resulted in an incubation of craving response and only the chocolate pellets elicited a CPP response, which degraded by the 14-day abstinence period. Labelling of c-Fos and FosB only differed from control at the 7-day abstinence period in the chocolate pellet group, neither chocolate group differed from each other at any abstinence period. Dendritic spines only differed between groups at the 28-day abstinence period. This differed from chapter II where increased dendritic spine density was seen across all abstinence periods in the classically conditioned rats. In the CPP study, rats only received food for five of the conditioning days compared to the 10 days 
in Chapter II and the food restricted controls also underwent exposure to the apparatus whereas in Chapter II they were kept in their home cage. These factors could have contributed to the lack of group differences as exposure to the apparatus itself could have influenced NAc activation in the control group.

Chapter IV built on Chapter II and III by exploring foods of different macronutrient compositions and flavors to determine if these factors affected responding for cues following periods of abstinence. Chapter IV also explored changes in AMPA receptor composition, which has been associated with dendritic spine changes (Rochefort and Konnerth, 2012) which we observed in Chapter II. Of the foods tested for an incubation of craving response in chapter IV, purified, sucrose, and chocolate flavored sucrose, only administration of purified food pellets was associated with an incubation of craving response. The purified group also displayed the highest responding during operant acquisition whereas the sucrose and chocolate sucrose groups did not significantly differ from each other. Expression of GluR1 time-dependently decreased in the purified and sucrose groups relative to control. Rats in all food groups that were tested for cue responding showed increased GluR1 expression compared to rats that underwent acquisition but not testing. In addition to chapter III, chapter IV highlighted how foods of different macronutrient composition can affect the magnitude of responding for cues previously associated with reward during forced abstinence. Chapter IV also showed that foods of similar macronutrient composition, sucrose and chocolate flavored sucrose, may differentially affect AMPA expression in the NAc.

The self-administration, classical conditioning, and CPP models utilized in the present thesis were able to produce behavioral learning with the exception of milk chocolate administration in the CPP model (chapter III). In Chapters II and IV, selfadministration in the presence of discrete cues was able to produce robust learning regardless of the food type. The type of food reward clearly affected the magnitude of responding during acquisition. This is not surprising as rats assigned to the sucrose and chocolate sucrose groups likely became satiated during the $1 \mathrm{hr}$ conditioning session. The magnitude of responding during acquisition may be a factor in the incubation of craving response in the present model as purified food pellets, which produced one of the highest responses during training, was the only food group to display an incubation response.

It is not unusual for an incubation of craving response to differ between reward types and methods of administration (i.e. contingent vs non-contingent). Incubation of craving has been consistently shown following abstinence from self-administered cocaine (Grimm et al., 2002; Lu et al., 2004) and liquid sucrose (Grimm et al., 2005). Noncontingent models are less consistent in eliciting an incubation response, for example following abstinence from morphine or cocaine in a CPP model, place preference has been shown to time-dependently decrease (Sun et al., 2017) or increase (Li et al., 2008) for morphine and increase for cocaine (Lubbers et al., 2015). We have shown that even if the same reward is utilized, chocolate flavored pellets, the outcome can differ depending on the behavioral model. In chapter II we noted consistent responding for cues over all abstinence periods whereas in chapter III, the CPP declined over time. This shows that the type of behavioral design may play a vital role in the magnitude of cue-responding following abstinence. 
In CPP models, the development, magnitude, and persistence of the response has been shown to depend on the quantity of reward and amount of conditioning sessions (Alderson et al., 2001; Brabant et al., 2005). When rats are testing for CPP to sucrose solutions of various percentages, 4,12 , and, 20, rats are unable to develop a CPP for the $4 \%$ solution (Alderson et al., 2001). The amount of conditioning sessions has been shown to affect the persistence of CPP following abstinence from cocaine (Brabant et al., 2005). For example, in rats that underwent conditioning for two sessions, the CPP ceased by 14 days, whereas rats conditioned for four sessions showed that the CPP was still present at 28 days and the magnitude of the effect was dose dependent (Brabant et al., 2005). Food has been shown to result in a lower magnitude CPP compared to drugs such as morphine and cocaine, possibly due to the different reinforcing properties of the substances (Baunez et al., 2005; Duarte et al., 2003; Tzschentke, 2007). For example, cocaine and amphetamine directly increase extracellular DA concentrations within the mesocorticolimbic DA system (Alonso-Alonso et al., 2015). Food reward also affects extracellular DA levels, but through multiple mechanisms that include neural input from taste buds, digestion, and hormones (Alonso-Alonso et al., 2015). We did not directly compare quantities in chapter III but we did compare foods of difference macronutrient composition which may have resulted in differential caloric intake. Future studies could explore the effect of quantity and conditioning sessions on the magnitude of CPP following periods of forced abstinence. It is probable that the amount of conditioning sessions and quantity of reward was not sufficient to produce long-lasting CPP.

In chapter II, the rats trained for classical conditioning (non-contingent) displayed lower levels of FosB and c-Fos labeling in the NAcSh compared to both control and operant trained rats. Extracellular DA release in the NAcSh has been shown to habituate over trials following non-contingent sucrose administration (Bassareo et al., 2015a). Since D1 receptor binding plays a role in the expression of immediate early genes, habitation of DA may result in decreased expression (Muller and Unterwald, 2005). In chapter III, a CPP model, which also consists of non-contingent administration, did not produce the same labeling patterns as seen in chapter II. It is plausible that in the CPP design, DA habituation in response to food reward does not occur or does not occur to the same degree as daily food administration. In the CPP design, rats only receive food reward on every other day of conditioning rather than everyday possibly preventing habituation of DA release.

The use of food restriction may have affected behavioral responding and neural changes in the models used in this thesis. Food restricted rats may engage in food seeking to restore homeostatic balance and avoid negative affect (Corwin and Hajnal, 2005). Food restriction has been shown to affect the mesolimbic DA system by decreasing extracellular DA, increasing DA reserves (Pothos et al., 1995), and altering DA receptor sensitivity (Haberny et al., 2004). Evidence for the functionality of NAc DA alterations following food restriction comes from studies with cocaine, where food restriction has been shown to amplify the drug response (Carr et al., 2000). Food restriction has also been shown to amplify release of DA in response to food (Ferrario et al., 2016). In chapters II and IV, it is possible that food restriction altered responding for cues behavior and prevented an incubation effect, with the exception of the purified group. The purified group was food restricted but overall daily food intake following 
acquisition sessions was similar to ad lib fed rats. The decreased consumption during abstinence would have been greater for the purified group and may have been a factor in the elicitation of an incubation response. In the CPP model, place preference was not seen beyond the 7-day abstinence period, but the limited differences in NAc responses between groups, including control, could have been a result of the effect of food restriction on the mesolimbic DA system.

Self-administration of food reward has been shown to increase dendritic spine density in the NAc (Guegan et al., 2013) and long-term changes in spine density have been associated with craving behavior and/or seeking behavior (Robinson and Kolb, 2004). Dendritic spine density was measured in chapters II and III and unexpectedly, despite the use of the same food reward, chocolate pellets, the pattern of spine density differed. In chapter II, both operant and classically conditioned rats displayed elevated spine density across all abstinence periods compared to controls. In chapter III, conditioning with chocolate flavored pellets only resulted in elevated spine density at the 28-day abstinence period, which was the time point when spine density decreased in the control group. The type of control group used in chapter II and III differed, which could have been a factor in the lack of spine density effects in chapter III. In chapter II, the control was food restricted but did not undergo any behavioral training or testing whereas in chapter III the control underwent the same conditioning procedure but did not receive food reward at any time. It is possible that exposure to the apparatus alone affected the dendritic spine density, thus this crucial difference cannot be discounted.

In chapter II, we showed that persistent responding for cues over all abstinence periods was associated with elevated spine density. We hypothesized that this may be the result of alterations in AMPA receptor expression, but in chapter IV, we showed that GluR1 expression decreased over time and there was no change in GluR2 expression. A time-dependent decrease in GluR1 expression was noted in the purified and sucrose food groups. Since only the purified group displayed an incubation of craving response, the functional significance of this remains elusive. Exposure to cues during testing rapidly increased GluR1 expression above rats that were not exposed to cues but there were no significant differences in GluR1 pSer 845 expression in any group. Since phosphorylation of GluR1 at Ser845 is involved in insertion of surface AMPA receptors (Carr, 2016) the AMPA receptors may not have been trafficked to the membrane, thus may not be functional. Another explanation for spine changes is alterations in NMDA receptor expression, which could be investigated in future studies.

The 7-day abstinence period was associated with changes in c-Fos, FosB, spine density, and GluR1 expression in all behavioral models. In chapters II and III, the chocolate flavored pellet group was more likely to differ from controls at this time point and in chapter II the 7-day abstinence period was associated with elevated dendritic spine density compared to 24 hrs. In Chapter IV, GluR1 expression decreased at the 7-day abstinence period compared to $24 \mathrm{hrs}$. in the purified and sucrose groups. It is plausible that at the 24-hr. abstinence period neural effects from the final day of conditioning are still measurable. Exposure to sucrose has been shown to rapidly elevate GluR1 expression in the NAc which can persist for 24 hrs. following exposure (Peng et al., 2011b). In future studies, measurement of NAc changes immediately after day 10 of conditioning would help elucidate this possibility. 
The NAcSh and NAcC have been proposed to have differential roles in conditioning and subsequent food seeking behavior. The NAcSh and NAcC have been proposed to differentially respond to novelty where NAcSh DA release is more likely to undergo habituation compared to the NAcC, an effect that may be exacerbated by food restriction (Chiara and Di Chiara, 2002). We differentiated the NAcSh and NAcC in the investigation of c-Fos, and FosB labeling in chapters II and III. In chapter II we did not observe any clear pattern differences between the NAcC and NAcSh in c-Fos labelling. For FosB, the 7-day period in the NAcSh in chapter II was associated with elevated labeling compared to $24 \mathrm{hrs}$. which was not seen in the NAcC. In chapter III, FosB labeling was similar between the NAcSh and NAcC. The lack of clear differences between NAcSh and NAcC neural effects makes it difficult to make clear comparisons between the two regions in the present thesis.

Future studies could further investigate adaptations in reward circuitry within the NAc in the behavioral models. Long-term changes in dendritic spines may result from adaptations in reward circuitry, specifically DA associated circuitry, during forced abstinence (Pickens et al., 2011; Sharma et al., 2013). These include changes in brainderived neurotrophic factor (BDNF), a neurotrophic factor that is important in the function of DA neurons and plays an important role in plasticity associated with learning and memory (Pickens et al., 2011; Sharma et al., 2013). Following five days of administration of amphetamine, BDNF has been shown to be increased in the NAc (Meredith et al., 2002). BDNF has also been shown to increase following 30 days, and continued to be elevated at 90 days, of cocaine abstinence in the NAc, but this effect was not seen for liquid sucrose (Grimm et al., 2003). High fat food has been shown to increase BDNF during withdrawal compared to BDNF prior to withdrawal (Sharma et al., 2013), but this does not take into account different abstinence periods (Grimm et al., 2003). These studies highlight that BDNF may be one of the factors in spine changes in incubation of craving studies as increases in BDNF occur at approximately the same time points where spine changes have been noted (Christian et al., 2016; Dingess et al., 2017b).

In chapter IV we were unable to determine the functional significance of the timedependent GluR1 expression changes. Future studies could address this via intraaccumbens injection with the CP-AMPA receptor antagonist NASPM prior to responding for cues testing. Following long periods of abstinence, when CP-AMPA receptor are increased, injection of NASPM has been shown to reduce responding for cues previously associated with cocaine (Wolf and Ferrario, 2010; Zheng et al., 2015). If the alterations in GluR1 expression seen in chapter IV have functional significance, when they are at their highest expression, NASPM would decrease responding for cues.

Further investigation into the effect of food restriction is paramount in understanding the structural and functional changes presented in the behavioral models in this thesis. Future studies should investigate these models in ad lib fed rats to determine if the same behavioral and neural effects are present. The effect of food restriction on DA activity within the NAc could also be explored by utilizing DA agonists prior to responding for cues testing. Since food restriction has been shown to alter behavioral responses to drugs that target the DA system (Carr et al., 2000), agonizing the DA system 
in both food restricted and ad lib fed rats may result in differential responding and elicit the functional significance that food restriction can have on DA activity.

\subsection{Conclusions}

The present thesis has shown that the food type and behavioral model can affect the possibility of an incubation of craving response and associated changes in the NAc. An incubation of craving response was limited to the purified food pellet group, whereas chocolate pellets, sucrose, and chocolate sucrose resulted in persistent responding across abstinence periods. Responding for cues associated with chocolate pellets was associated with elevated spine density highlighting that both behavioral and neural learning occurred. The behavioral effects of CPP were minimal and only the chocolate pellet group, not the milk chocolate group, displayed a place preference, but an incubation effect was not observed. Since contingent and non-contingent administration can differentially activate the NAc, this may be a factor in the time-dependent decrease in place preference during abstinence. These results raise the possibility that food type may be an important factor in eliciting a place preference and subsequent incubation response. The effect of food restriction may have altered food seeking behavior in the presented behavioral models and the effect of food-restriction alone on NAc activity cannot be overlooked. Changes in AMPA protein expression were not specific to the purified group, which displayed an incubation response, thus the functional significance has yet to be elucidated. Since the chocolate sucrose group did not display the same timedependent pattern of GluR1 expression as sucrose it suggests that flavor may be a factor in NAc responses. Responding for cues previously associated with reward resulted in a rapid increase in GluR1 expression and does not depend on food type. Further investigations which assess the contribution of food restriction to the present behavioral and neural outcomes and the functional significance of GluR1 time-dependent changes are needed to thoroughly evaluate incubation of craving responses using the presented behavioral models. 


\section{Chapter 6}

\section{Appendix A- Copyright Documentation}

\subsection{Permission from Frontiers in Behavioural Neuroscience}

Extracted from the Frontiers website (https://www.frontiersin.org/legal/copyrightstatement):

All content included on Frontiers websites (including Loop), such as text, graphics, logos, button icons, images, video/audio clips, downloads, data compilations and software, is the property of Frontiers if created by Frontiers, or of the person or entity who or which owned it prior to submission to Frontiers. If not owned by Frontiers, it is licensed to Frontiers Media SA (Frontiers) or its licensees and/or subcontractors.

The copyright in the text of individual articles (including research articles, opinion articles, book reviews, conference proceedings and abstracts) is not the property of Frontiers, and its ownership is not affected by its submission to or publication by Frontiers. Frontiers benefits from a general licence over all content submitted to it, and both Frontiers and its users benefit from a Creative Commons CC-BY licence over all content, as specified below.

Images and graphics not forming part of user-contributed materials are the property of or are licensed to Frontiers may not be downloaded or copied without Frontiers' explicit and specific permission or in accordance with any specific copyright notice attached to that material.

The combination of all content on Frontiers websites, as well as the design and the look and feel of the Frontiers websites, and the copyright and all other rights in such content and combination, are the sole property of Frontiers.

As an author or contributor you grant permission to others to reproduce your articles, including any graphics and third-party materials supplied by you, in accordance with the Frontiers Terms and Conditions. The licence granted to third parties over all contents of each article, including third-party elements, is a Creative Commons Attribution ("CC BY") licence. The current version is CC-BY, version 4.0, and the licence will automatically be updated as and when updated by the Creative Commons organisation.

You may include a requirement to reproduce copyright notices in materials contributed by you, but you may not restrict the right to reproduce the entire article, including thirdparty graphics. This means that you must obtain any necessary third-party consents and permissions to reproduce third-party materials in your articles submitted to Frontiers. 
E-books are subject to the same licensing conditions as the articles within them. Articles published prior to the effective date of this notice: Please note that reproduction of third-party graphics and other third-party materials contained in articles published prior to the effective date of this notice may be subject to third-party notices prohibiting their reproduction without permission. You must comply with those notices. Articles published prior to July 2012: The licence granted for these articles may be different and you should check the pdf version of any article to establish what licence was granted. If an article, dating from before July 2012, carries only a non-commercial licence and you wish to obtain a commercial licence, please contact Frontiers at editorial.office@,frontiersin.org.

All software used on this website, and the copyright in the code constituting such software, is the property of or is licensed to Frontiers and its use is restricted in accordance with the Frontiers Terms and Conditions. All copyright, and all rights therein, are protected by national and international copyright laws.

This Copyright Statement comes into effect on 25th May, 2018. 


\subsection{Co-author Permission Statements}

To whom is may concern:

I, Matthew Holahan, hereby grant Erin Noye Tuplin permission to use all contents associated with the manuscript, Comparison of the time-dependent changes in immediate early gene labeling and spin density following abstinence from contingent or noncontingent chocolate pellet delivery, published in Frontiers in Behavioural Neuroscience, as part of her $\mathrm{PhD}$ dissertation.

Signature:

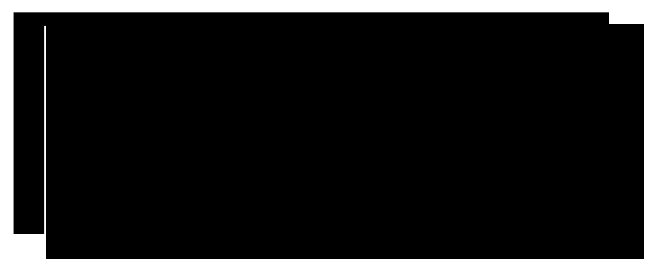

Date: July 29, 2018 
To whom is may concern:

I, Savannah Lightfoot, hereby grant Erin Noye Tuplin permission to use all contents associated with the manuscript, Comparison of the time-dependent changes in immediate early gene labeling and spin density following abstinence from contingent or non-contingent chocolate pellet delivery, published in Frontiers in Behavioural Neuroscience, as part of her $\mathrm{PhD}$ dissertation.

Signature:

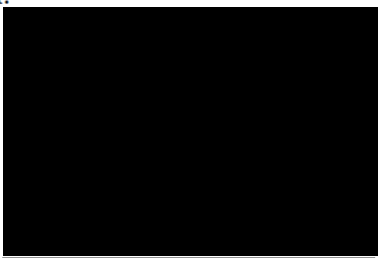

Date: July 30th 2018 
To whom is may concern:

I, Matthew Holahan, hereby grant Erin Noye Tuplin permission to use all contents associated with the manuscript, Exploring time-dependent changes in conditioned place preference for food reward and associated changes in the nucleus accumbens, to be submitted to Behavioural Brain Research, as part of her PhD dissertation.

Signature:

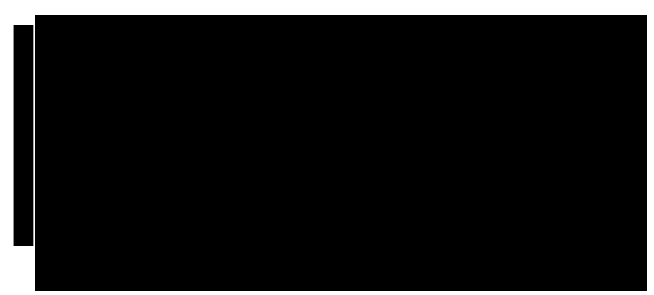

Date: July 29, 2018

To whom is may concern:

I, Matthew Holahan, hereby grant Erin Noye Tuplin permission to use all contents associated with the manuscript, Time-dependent changes in GluR1 protein expression following abstinence from food rewards of different macronutrient composition, to be submitted to Neuroscience, as part of her PhD dissertation.

Signature:

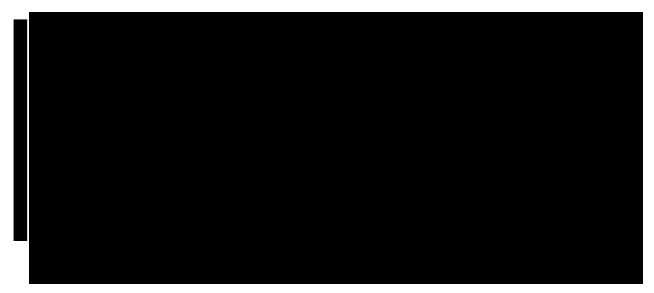

Date: July 29, 2018 
To whom is may concern:

I, Madi Wright, hereby grant Erin Noye Tuplin permission to use all contents associated with the manuscript, Time-dependent changes in GluRl protein expression following abstinence from food rewards of different macronutrient composition, to be submitted to Neuroscience, as part of her $\mathrm{PhD}$ dissertation.

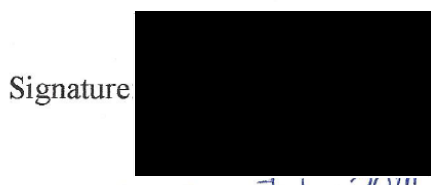

Date: Monday July 30 ,' 2018 


\title{
Chapter 7
}

\section{Appendix B-Supervisor's Statement}

\author{
Carleton \\ Canada's Capital University
}

July 29, 2018

I certify that this thesis conforms in all respects to the regulations of Carleton University and that I have verified the following requirements:

1. The thesis comprises original work which the student was fully involved in setting up and conducting. Erin not only set up and conducted all work reported in a largely independent manner (with guidance provided by me and minor assistance provided by students), she also collected and analyzed all data.

2. The student provided the conception and design of the experiments, provided analysis and interpretation of data, drafted and revised the manuscripts and submitted them serving as corresponding author and dealing with reviewer comments. The thesis supervisor edited written text, provided final approval for submitted and revised manuscripts and provided overall guidance in data analysis and interpretation.

3. The information provided by the student in the preface is accurate.

4. The thesis reflects work done during the period of candidature and no work presented has contributed to an award of a previous qualification.

5. Research data collected, used and maintained in the conduct of this thesis have been deposited with the supervisor having administrative oversight of the candidature in accordance with legal and ethical requirements. Research data and records will be retained and accessible for a minimum of 5 years from the date the thesis is passed or from the date of publication.

Sincerely, 


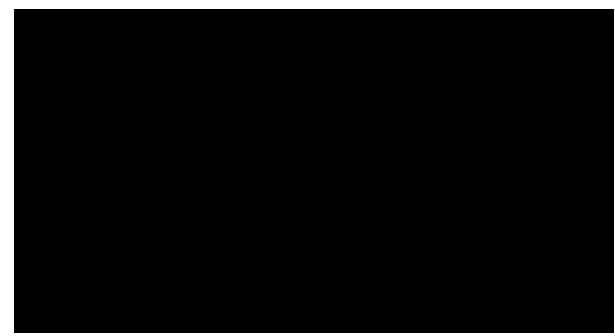

Matthew R. Holahan, PhD

Dept of Neuroscience/ Carleton University

5307 Health Sciences Building

1125 Colonel By Drive

Ottawa, ON Canada K1S 5B6

tele: 613-520-2600 ext 1543

Matthew.Holahan@carleton.ca 
Chapter 8

Appendix B-Western Blots

GluR2 protein:
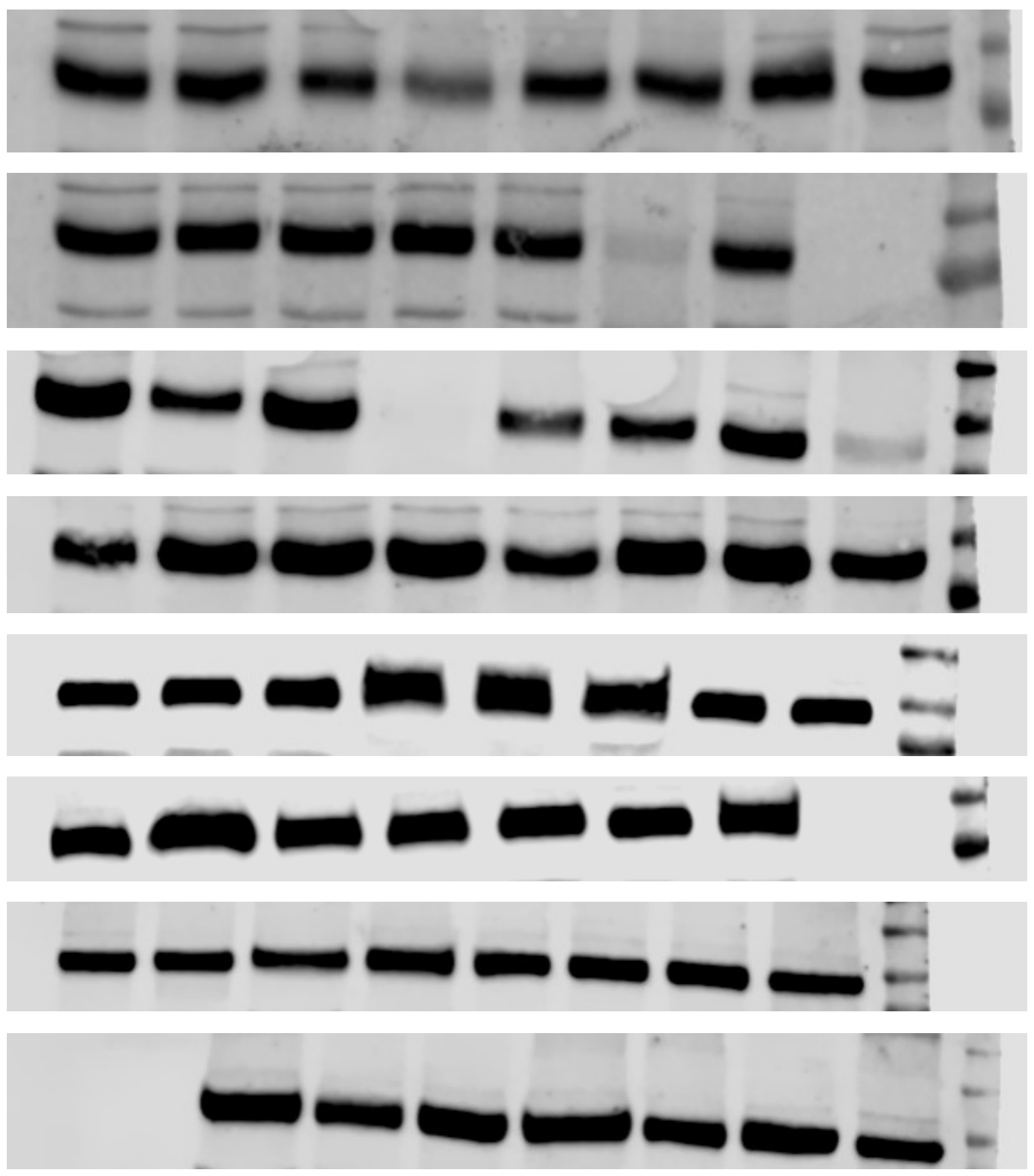

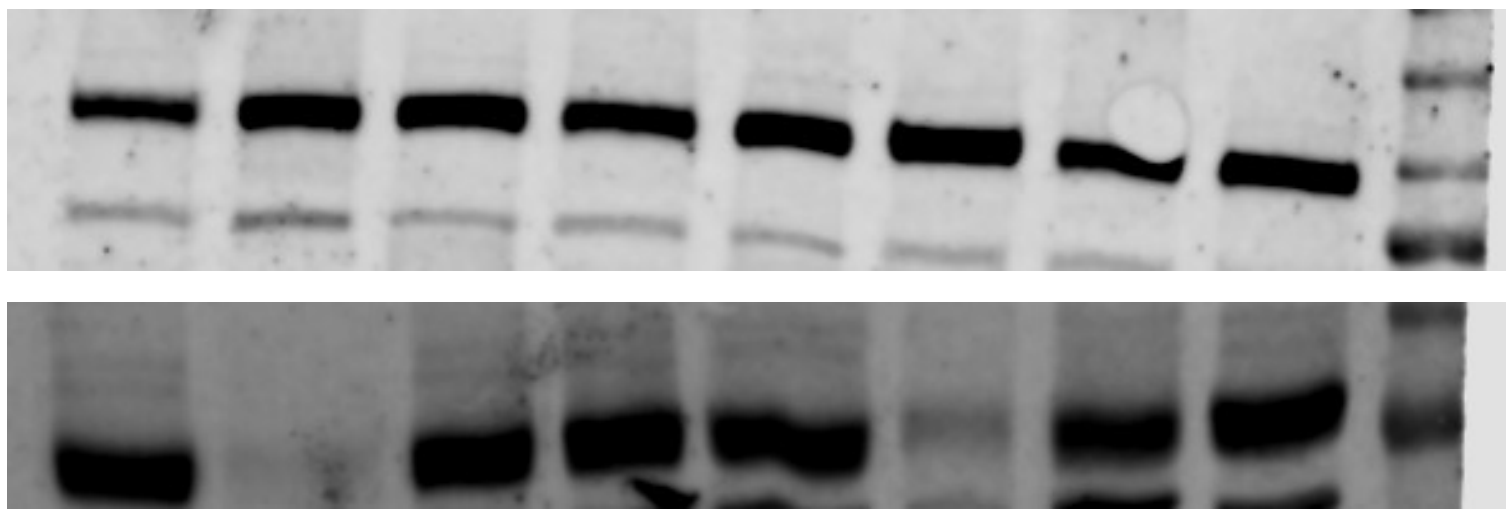
GluR1 protein:
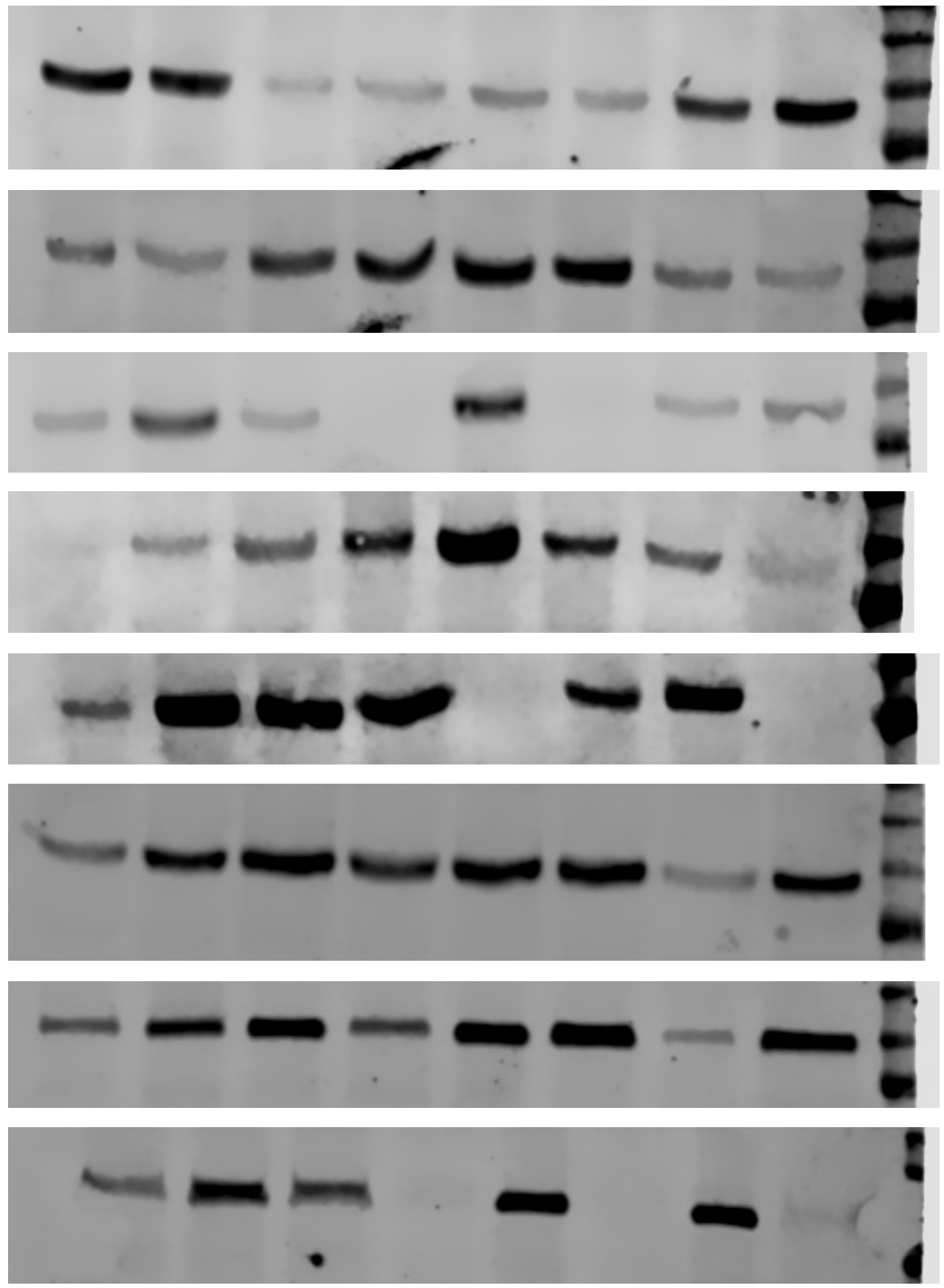

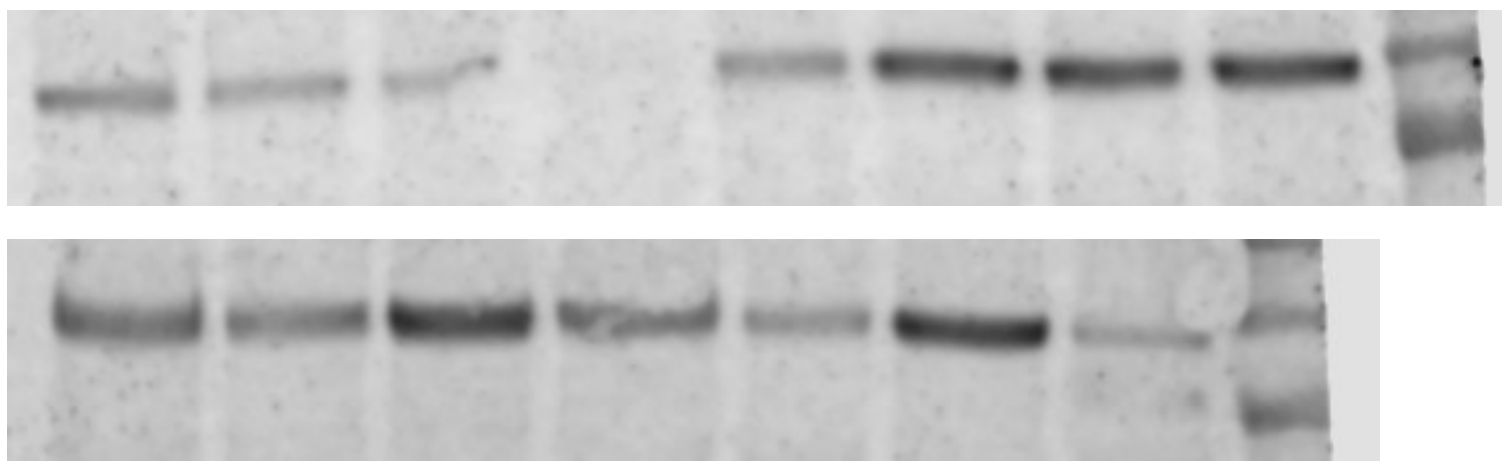
GluR1 pSer845:
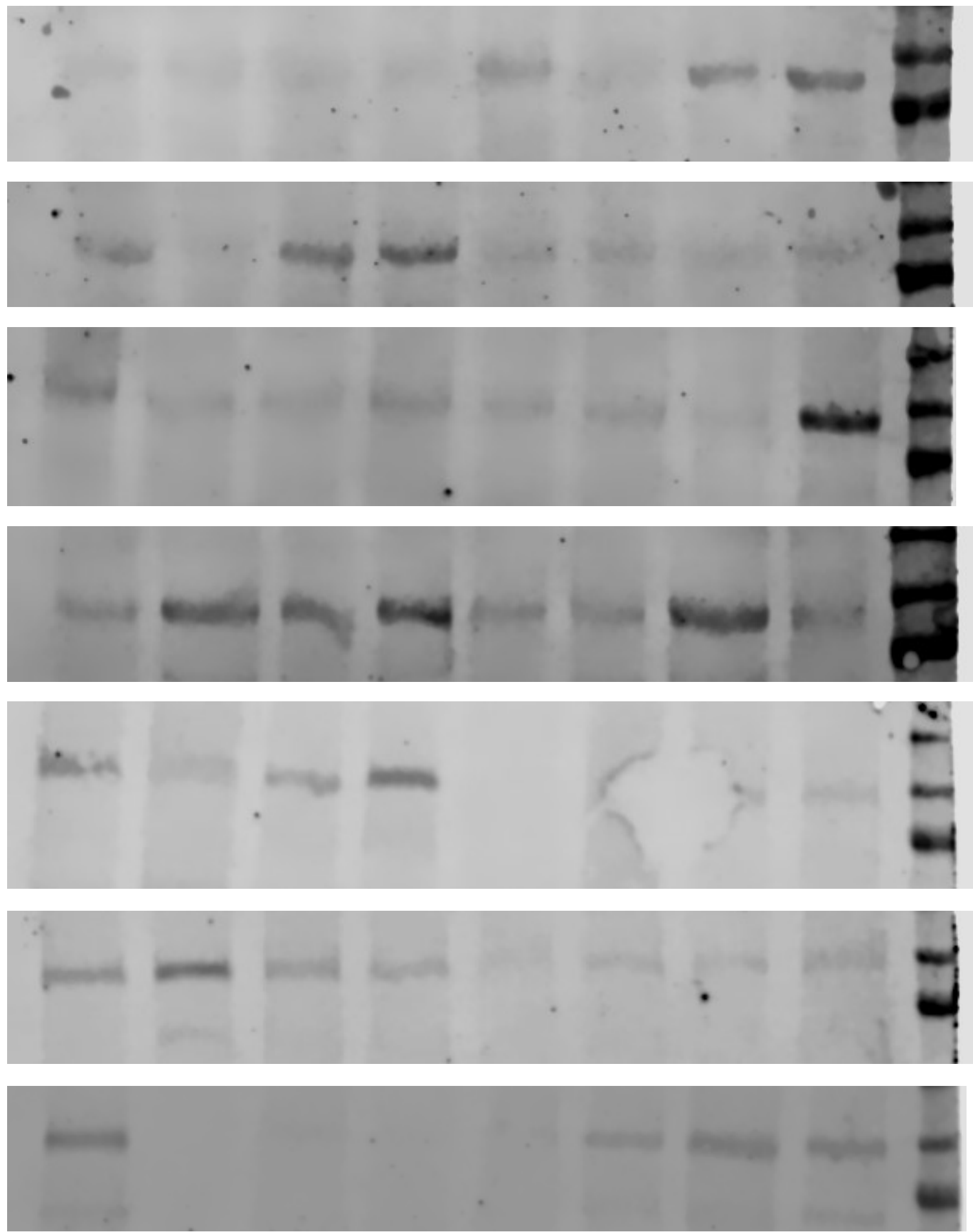

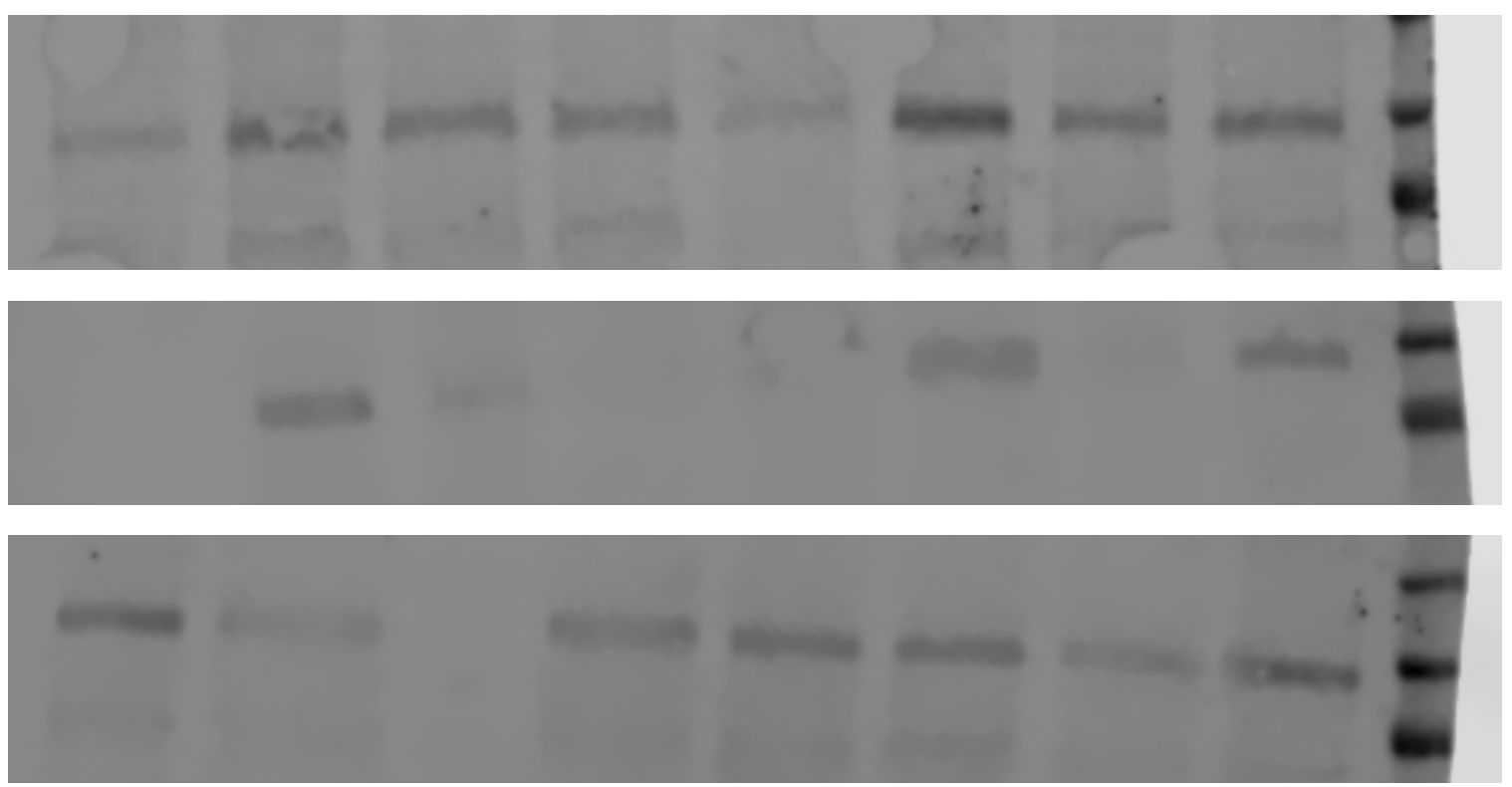


\section{References}

Abdolahi, A., Acosta, G., Breslin, F. J., Hemby, S. E., and Lynch, W. J. (2010). Incubation of nicotine seeking is associated with enhanced protein kinase Aregulated signaling of dopamine- and cAMP-regulated phosphoprotein of $32 \mathrm{kDa}$ in the insular cortex. Eur. J. Neurosci. 31, 733-741. doi:10.1111/j.14609568.2010.07114.x.

Adinoff, B. (2004). Neurobiologic Processes in Drug Reward and Addiction. Harv. Rev. Psychiatry 12, 305-320. doi:10.1080/10673220490910844.

Alderson, H. L., Jenkins, T. A., Kozak, R., Latimer, M. P., and Winn, P. (2001). The effects of excitotoxic lesions of the pedunculopontine tegmental nucleus on conditioned place preference to $4 \%, 12 \%$ and $20 \%$ sucrose solutions. Brain Res. Bull. 56, 599-605. doi:10.1016/S0361-9230(01)00733-X.

Alonso-Alonso, M., Woods, S. C., Pelchat, M., Grigson, P. S., Stice, E., Farooqi, S., et al. (2015). Food reward system: Current perspectives and future research needs. Nutr. Rev. 73, 296-307. doi:10.1093/nutrit/nuv002.

Aoyama, K., Barnes, J., and Grimm, J. W. (2014). Incubation of saccharin craving and within-session changes in responding for a cue previously associated with saccharin. Appetite 72, 114-122. doi:10.1016/j.appet.2013.10.003.

Avena, N. M., Rada, P., and Hoebel, B. G. (2008). Evidence for sugar addiction: Behavioral and neurochemical effects of intermittent, excessive sugar intake. Neurosci. Biobehav. Rev. 32, 20-39. doi:10.1016/j.neubiorev.2007.04.019.

Baik, J.-H. (2013a). Dopamine Signaling in reward-related behaviors. Front. Neural Circuits 7, 1-16. doi:10.3389/fncir.2013.00152.

Baik, J. H. (2013b). Dopamine signaling in food addiction: Role of dopamine D2 receptors. BMB Rep. 46, 519-526. doi:10.5483/BMBRep.2013.46.11.207.

Bardo, M., and Hammer, R. P. (1991). Autoradiographic localization of dopamine D1 and D2 receptors in rat nucleus accumbens: Resistance to differential rearing conditions. Neuroscience 45, 281-290. doi:10.1016/0306-4522(91)90226-E.

Bardo, M. T., and Bevins, R. A. (2000). Conditioned place preference: What does it add to our preclinical understanding of drug reward? Psychopharmacology (Berl). 153, 31-43. doi:10.1007/s002130000569.

Barry, D., Clarke, M., and Petry, N. M. (2010). Obesity and its relationship to addictions: is overeating a form of addictive behavior? Am. J. Addict. 18, 439-451. doi:10.3109/10550490903205579.

Bassareo, V., Cucca, F., Frau, R., and Di Chiara, G. (2015a). Differential activation of accumbens shell and core dopamine by sucrose reinforcement with nose poking and with lever pressing. Behav. Brain Res. 294, 215-223.

doi:10.1016/j.bbr.2015.08.006. 
Bassareo, V., Cucca, F., Musio, P., Lecca, D., Frau, R., and Di Chiara, G. (2015b). Nucleus accumbens shell and core dopamine responsiveness to sucrose in rats: Role of response contingency and discriminative/conditioned cues. Eur. J. Neurosci. 41, 802-809. doi:10.1111/ejn.12839.

Bassareo, V., and Di Chiara, G. (1997). Differential influence of associative and nonassociative learning mechanisms on the responsiveness of prefrontal and accumbal dopamine transmission to food stimuli in rats fed ad libitum. J Neurosci 17, 851-861. doi:10.1177/1087054705277198.

Bassareo, V., and Di Chiara, G. (1999). Differential responsiveness of dopamine transmission to food-stimuli in nucleus accumbens shell/core compartments. Neuroscience 89, 637-641. doi:10.1016/S0306-4522(98)00583-1.

Baunez, C., Dias, C., Cador, M., and Amalric, M. (2005). The subthalamic nucleus exerts opposite control on cocaine and "natural" rewards. Nat. Neurosci. 8, 484-489. doi:10.1038/nn1429.

Beaulieu, J., and Gainetdinov, R. R. (2011). The Physiology, Signaling, and Pharmacology of Dopamine Receptors. Pharmacol. Rev. 63, 182-217. doi:10.1124/pr.110.002642.182.

Beeler, J. A., Frazier, Christianne, R. M., and Zhuang, X. (2012). Dopaminergic enhancement of local food seeking is under global homeostatic control. Eur. J. Neurosci. 35, 146-149. doi:10.1111/j.1743-6109.2008.01122.x.Endothelial.

Belin-Rauscent, A., Fouyssac, M., Bonci, A., and Belin, D. (2015). How preclinical models evolved to resemble the diagnostic criteria of drug addiction. Biol. Psychiatry, 1-8. doi:10.1016/j.biopsych.2015.01.004.

Berretta, S., Pantazopoulos, H., M., C., Pantazopoulos, P., and Pare, D. (2005). Infralimbic cortex activation increases c-fos expression in intercalated neurons of the amygdala. Neuroscience 132, 943-953.

Bevins, R. a, Klebaur, J. E., and Bardo, M. T. (1997). Individual differences in response to novelty, amphetamine-induced activity and drug discrimination in rats. Behav. Pharmacol. 8, 113-23. Available at: http://www.ncbi.nlm.nih.gov/pubmed/9833007.

Bienkowski, P., Rogowski, A., Korkosz, A., Mierzejewski, P., Radwanska, K., Kaczmarek, L., et al. (2004). Time-dependent changes in alcohol-seeking behaviour during abstinence. Eur. Neuropsychopharmacol. 14, 355-360. doi:10.1016/j.euroneuro.2003.10.005.

Björklund, A., and Dunnett, S. B. (2007). Dopamine neuron systems in the brain: an update. Trends Neurosci. 30, 194-202. doi:10.1016/j.tins.2007.03.006.

Blancas, A., Gonzalez-Garcia, S. D., Rodriguez, K., and Escobar, C. (2014). Progressive anticipation in behavior and brain activation of rats exposed to scheduled daily palatable food. Neuroscience 281, 44-53. doi:10.1016/j.neuroscience.2014.09.036.

Bossert, J. M., Ghitza, U. E., Lu, L., Epstein, D. H., and Shaham, Y. (2005). Neurobiology of relapse to heroin and cocaine seeking: An update and clinical 
implications. Eur. J. Pharmacol. 526, 36-50. doi:10.1016/j.ejphar.2005.09.030.

Bourne, J., and Harris, K. M. (2007). Do thin spines learn to be mushroom spines that remember? Curr. Opin. Neurobiol. 17, 381-386. doi:10.1016/j.conb.2007.04.009.

Brabant, C., Quertemont, E., and Tirelli, E. (2005). Influence of the dose and the number of drug-context pairings on the magnitude and the long-lasting retention of cocaineinduced conditioned place preference in C57BL/6J mice. Psychopharmacology (Berl). 180, 33-40. doi:10.1007/s00213-004-2138-6.

Brandon, T. H., Vidrine, J. I., and Litvin, E. B. (2007). Relapse and relapse prevention. Annu. Rev. Clin. Psychol. 3, 257-284. doi:10.1146/annurev.clinpsy.3.022806.091455.

Breiter, H. C., Gollub, R. L., Weisskoff, R. M., Kennedy, D. N., Markris, N., Berke, J. D., et al. (1997). Acute effects of cocaine on human brain activity and emotion. Neuron 19, 591-611. Available at: http://www.ncbi.nlm.nih.gov/htbinpost $/$ Entrez/query?db=m\&form=6\&dopt=r\&uid=9331351.

Britton, D. R., Curzon, P., Mackenzie, R. G., Kebabian, J. W., Williams, J. E. G., and Kerkman, D. (1991). Evidence for involvement of both D1 and D2 receptors in maintaining cocaine self-administration. Pharmacol. Biochem. Behav. 39, 911-915. doi:10.1016/0091-3057(91)90052-4.

Bruinsma, K., and Taren, D. L. (1999). Chocolate: Food or Drug? J. Am. Diet. Assoc. 99, $1249-1256$.

Burger, K. S., and Stice, E. (2011). Variability in reward responsivity and obesity: evidence from brain imaging studies. Curr. Drug Abuse Rev. 4, 182-9. doi:10.2174/1874473711104030182.

Butz, M., Wörgötter, F., and van Ooyen, A. (2009). Activity-dependent structural plasticity. Brain Res. Rev. 60, 287-305. doi:10.1016/j.brainresrev.2008.12.023.

Cabeza de Vaca, S., and Carr, K. D. (1998). Food restriction enhances the central rewarding effect of abused drugs. J. Neurosci. 18, 7502-7510.

Cabib, S., Castellano, C., Cestari, V., Filibeck, U., and Puglisi-Allegra, S. (1991). D1 and D2 receptor antagonists differently affect cocaine-induced locomotor hyperactivity in the mouse. Psychopharmacology (Berl). 105, 335-339.

Caine, S. B., and Koob, G. F. (1994). Effects of dopamine D1 and D2 antagonists on cocaine self-administration under different schedules of reinforcement in the rat. $J$. Pharmacol. Exp. Ther. 270, 209-218.

Caine, S. B., Thomsen, M., Gabriel, K. I., Berkowitz, J. S., Gold, L. H., Koob, G. F., et al. (2007). Lack of self-administration of cocaine in dopamine D1 receptor knockout mice. J. Neurosci. 27, 12140-13150.

doi:10.1016/j.humov.2008.02.015.Changes.

Calu, D. J., Stalnaker, T. a, Franz, T. M., Singh, T., Shaham, Y., and Schoenbaum, G. (2007). Withdrawal from cocaine self-administration produces long-lasting deficits 
in orbitofrontal-dependent reversal learning in rats. Learn. Mem. 14, 325-328. doi:10.1101/lm.534807.

Canadian Institute for Health Information (2011). Obesity in Canada.

Canan, F., Karaca, S., Sogucak, S., Gecici, O., and Kuloglu, M. (2017). Eating disorders and food addiction in men with heroin use disorder: a controlled study. Eat. Weight Disord. 22, 249-257. doi:10.1007/s40519-017-0378-9.

Caprioli, D., Venniro, M., Zeric, T., Li, X., Adhikary, S., Madangopal, R., et al. (2015). Effect of the novel positive allosteric modulator of metabotropic glutamate receptor 2 AZD8529 on incubation of methamphetamine craving after prolonged voluntary abstinence in a rat model. Biol. Psychiatry 78, 463-473. doi:10.1016/j.biopsych.2015.02.018.

Caprioli, D., Venniro, M., Zhang, M., Bossert, J. M., Warren, B. L., Hope, B. T., et al. (2017). Role of Dorsomedial Striatum Neuronal Ensembles in Incubation of Methamphetamine Craving after Voluntary Abstinence. J. Neurosci. 37, 1014-1027. doi:10.1523/JNEUROSCI.3091-16.2017.

Carelli, R. M., and Deadwyler, S. A. (1994). A Comparison of Nucleus Accumbens Neuronal Firing Patterns during Cocaine Self-Administration and Water Reinforcement in Rats. J. Neurosci. 14, 7735-7746.

Carr, K. D. (2016). Nucleus accumbens AMPA receptor trafficking upregulated by food restriction: An unintended target for drugs of abuse and forbidden foods. Curr. Opin. Behav. Sci. 9, 32-39. doi:10.1016/j.cobeha.2015.11.019.

Carr, K. D., Chau, L. S., Cabeza de Vaca, S., Gustafson, K., Stouffer, M., Tukey, D. S., et al. (2010). AMPA receptor subunit GluR1 downstream of D-1 dopamine receptor stimulation in nucleus accumbens shell mediates increased drug reward magnitude in food-restricted rats. Neuroscience 165, 1074-1086.

doi:10.1016/j.neuroscience.2009.11.015.

Carr, K. D., Kim, G. Y., and Cabeza de Vaca, S. (2000). Chronic food restriction in rats augments the central rewarding effect of cocaine and the $\delta 1$ opioid agonist, DPDPE, but not the $\delta 2$ agonist, deltorphin-II. Psychopharmacology (Berl). 152, 200-207. doi:10.1007/s002130000523.

Carr, K. D., Tsimberg, Y., Berman, Y., and Yamamoto, N. (2003). Evidence of increased dopamine receptor signaling in food-restricted rats. Neuroscience 119, 1157-1167. doi:10.1016/S0306-4522(03)00227-6.

Caster, J. M., and Kuhn, C. M. (2009). Maturation of coordinated immediate early gene expression by cocaine during adolescence. Neuroscience 160, 13-31. doi:10.1016/j.neuroscience.2009.01.001.

Chao, J., and Nestler, E. J. (2004). Molecular Neurobiology of Drug Addiction. Annu. Rev. Med. 55, 113-132. doi:10.1146/annurev.med.55.091902.103730.

Chaudhri, N., Sahuque, L. L., Schairer, W. W., and Janak, P. H. (2010). Separable roles of the nucleus accumbens core and shell in context- and cue-induced alcohol- 
seeking. Neuropsychopharmacology 35, 783-791. doi:10.1038/npp.2009.187.

Chauvet, C., Goldberg, S. R., Jaber, M., and Solinas, M. (2012). Effects of environmental enrichment on the incubation of cocaine craving. Neuropharmacology 63, 635-641. doi:10.1016/j.neuropharm.2012.05.014.

Chauvet, C., Lardeux, V., Goldberg, S. R., Jaber, M., and Solinas, M. (2009). Environmental enrichment reduces cocaine seeking and reinstatement induced by cues and stress but not by cocaine. Neuropsychopharmacology 34, 2767-2778. doi:10.1038/npp.2009.127.

Chen, J.-C., Chen, P.-C., and Chiang, Y.-C. (2009). Molecular Mechanisms of Psychostimulant Addiction. Chang Gung Med. J. 32, 148-154. doi:3202/320204 [pii].

Chen, K., Yan, J., Li, J., Lv, B., and Zhao, X. (2011). c-Fos expression in rat brainstem following intake of sucrose or saccharin. Front. Med. China 5, 294-301. doi:10.1007/s11684-011-0144-8.

Chiara, G. Di (2002). Nucleus accumbens shell and core dopamine: differential role in behavior and addiction. Behav. Brain Res. 137, 75-114.

Chiara, G. Di, and Di Chiara, G. (2002). Nucleus accumbens shell and core dopamine: differential role in behavior and addiction. Behav. Brain Res. 137, 75-114. doi:10.1016/S0166-4328(02)00286-3.

Childress, A. R., Mozley, P. D., McElgin, W., Fitzgerald, J., Reivich, M., and O'Brien, Charles, P. (1999). Limbic Activation During Cue-Induced Cocaine Craving. Am. J. Psychiatry 156, 997-1003. doi:10.1016/j.biotechadv.2011.08.021.Secreted.

Christian, D. T., Wang, X., Chen, E. L., Sehgal, L. K., Ghassemlou, M. N., Miao, J. J., et al. (2016). Dynamic Alterations of Rat Nucleus Accumbens Dendritic Spines over two Months of Abstinence from Extended-Access Cocaine Self-Administration. Neuropsychopharmacology 42, 1-29. doi:10.1038/npp.2016.168.

Citri, A., and Malenka, R. C. (2008). Synaptic Plasticity: Multiple Forms, Functions, and Mechanisms. Neuropsychopharmacology 33, 18-41. doi:10.1038/sj.npp.1301559.

Colagiuri, B., and Lovibond, P. F. (2015). How food cues can enhance and inhibit motivation to obtain and consume food. Appetite 84, 79-87.

doi:10.1016/j.appet.2014.09.023.

Colbran, R. J. (2015). Thematic minireview series: Molecular mechanisms of synaptic plasticity. J. Biol. Chem. 290, 28594-28595. doi:10.1074/jbc.R115.696468.

Colby, C. R., Whisler, K., Steffen, C., Nestler, E. J., and Self, D. W. (2003). Striatal cell type-specific overexpression of DeltaFosB enhances incentive for cocaine. $J$. Neurosci. 23, 2488-2493. doi:23/6/2488 [pii].

Conrad, K. L., Tseng, K. Y., Uejima, J. L., Reimers, J. M., Heng, L.-J., Shaham, Y., et al. (2008). Formation of accumbens GluR2-lacking AMPA receptors mediates incubation of cocaine craving. Nature 454, 118-21. doi:10.1038/nature06995. 
Cornish, J. L., and Kalivas, P. W. (2000). Glutamate transmission in the nucleus accumbens mediates relapse in cocaine addiction. J. Neurosci. 20, RC89. doi:20004403 [pii].

Corwin, R. L., and Hajnal, A. (2005). Too much of a good thing: Neurobiology of nonhomeostatic eating and drug abuse. Physiol. Behav. 86, 5-8. doi:10.1016/j.physbeh.2005.06.021.

Counotte, D. S., Schiefer, C., Shaham, Y., and O’Donnell, P. (2014). Time-dependent decreases in nucleus accumbens AMPA/NMDA ratio and incubation of sucrose craving in adolescent and adult rats. Psychopharmacology (Berl). 231, 1675-1684. doi:10.1007/s00213-013-3294-3.

Crocq, M. A. (2007). Historical and cultural aspects of man's relationship with addictive drugs. Dialogues Clin. Neurosci. 9, 355-361.

Cruz, F. C., Javier Rubio, F., and Hope, B. T. (2015). Using c-fos to study neuronal ensembles in corticostriatal circuitry of addiction. Brain Res. 1628, 157-173. doi:10.1016/j.brainres.2014.11.005.

Darling, R. A., Dingess, P. M., Schlidt, K. C., Smith, E. M., and Brown, T. E. (2016). Incubation of food craving is independent of macronutrient composition. Sci. Rep. 6, 30900. doi:10.1038/srep30900.

Davis-MacNevin, P. L., Dekraker, J., LaDouceur, L., and Holahan, M. R. (2013). Comparison of the MK-801-induced increase in non-rewarded appetitive responding with dopamine agonists and locomotor activity in rats. J. Psychopharmacol. 27, 854-64. doi:10.1177/0269881113492029.

Davis, C., and Carter, J. C. (2009). Compulsive overeating as an addiction disorder. A review of theory and evidence. Appetite 53, 1-8. doi:10.1016/j.appet.2009.05.018.

Davis, C., Curtis, C., Levitan, R. D., Carter, J. C., Kaplan, A. S., and Kennedy, J. L. (2011). Evidence that "food addiction" is a valid phenotype of obesity. Appetite 57, 711-717. doi:10.1016/j.appet.2011.08.017.

Day, J. J., and Carelli, R. M. (2007). The Nucleus Accumbens and Pavlovian Reward Learning. Neuroscientist 13, 148-159. doi:10.1177/1073858406295854.The.

De Jong, J. W., Vanderschuren, L. J. M. J., and Adan, R. A. H. (2012). Towards an animal model of food addiction. Obes. Facts 5, 180-195. doi:10.1159/000338292.

Dela Cruz, J. A. D., Coke, T., Karagiorgis, T., Sampson, C., Icaza-Cukali, D., Kest, K., et al. (2015). C-Fos induction in mesotelencephalic dopamine pathway projection targets and dorsal striatum following oral intake of sugars and fats in rats. Brain Res. Bull. 111, 9-19. doi:10.1016/j.brainresbull.2014.11.002.

Deutch, A. Y., Goldstein, M., Balding, F., and Roth, R. H. (1988). Telencephalic Projections of the A8 Dopamine Cell Group. Ann. N. Y. Acad. Sci. 537, 27-50. doi:10.1111/j.1749-6632.1988.tb42095.x.

Di Chiara, G. (2002). Nucleus accumbens shell and core dopamine: Differential role in 
behavior and addiction. Behav. Brain Res. 137, 75-114. doi:10.1016/S01664328(02)00286-3.

Di Chiara, G., Bassareo, V., Fenu, S., De Luca, M. A., Spina, L., Cadoni, C., et al. (2004). Dopamine and drug addiction: the nucleus accumbens shell connection. Neuropharmacology 47 Suppl 1, 227-41. doi:10.1016/j.neuropharm.2004.06.032.

Diehl, G. W., Wachtel, J. M., and Paine, T. A. (2013a). Cue-induced conditioned activity does not incubate but is mediated by the basolateral amygdala. Pharmacol. Biochem. Behav. 104, 69-79. doi:10.1016/j.pbb.2013.01.003.

Diehl, G. W., Wachtel, J. M., and Paine, T. A. (2013b). Cue-induced conditioned activity does not incubate but is mediated by the basolateral amygdala. Pharmacol. Biochem. Behav. 104, 69-79. doi:10.1016/j.pbb.2013.01.003.

Dingess, P. M., Darling, R. A., Derman, R. C., Wulff, S. S., Hunter, M. L., Ferrario, C. R., et al. (2017a). Structural and Functional Plasticity within the Nucleus Accumbens and Prefrontal Cortex Associated with Time-Dependent Increases in Food Cue-Seeking Behavior. Neuropsychopharmacology 42, 2354-2364. doi:10.1038/npp.2017.57.

Dingess, P. M., Darling, R. A., Derman, R. C., Wulff, S. S., Hunter, M. L., Ferrario, C. R., et al. (2017b). Structural and Functional Plasticity within the Nucleus Accumbens and Prefrontal Cortex Associated with Time-Dependent Increases in Food Cue Seeking Behavior. Neuropsychopharmacology, 1-45. doi:10.1038/npp.2017.57.

Domjan, M. (2005). Pavlovian conditioning: a functional perspective. Annu. Rev. Psychol. 56, 179-206. doi:10.1146/annurev.psych.55.090902.141409.

Drummond, D. C. (2001). Theories of drugs craving, ancient and modern. Addiction 96, 33-46. doi:10.1080/09652140020016941.

DSM-V (2013). Diagnostic and Statistical Manual of mental disorders (5th ed.). 6th ed. Washington DC.

Duan, Y., Shen, F., Gu, T., and Sui, N. (2016). Addiction: From context-induced hedonia to appetite, based on transition of micro-behaviors in morphine abstinent tree shrews. Front. Psychol. 7, 1-9. doi:10.3389/fpsyg.2016.00816.

Duarte, C., Lefebvre, C., Chaperon, F., Hamon, M., and Thiébot, M. H. (2003). Effects of a Dopamine D3Receptor Ligand, BP 897, on Acquisition and Expression of Food-, Morphine-, and Cocaine-induced Conditioned Place Preference, and Food-seeking Behavior in Rats. Neuropsychopharmacology 28, 1903-1915. doi:10.1038/sj.npp.1300276.

Duarte, R. B. M., Patrono, E., Borges, A. C., C??sar, A. A. S., Tomaz, C., Ventura, R., et al. (2014). Consumption of a highly palatable food induces a lasting placeconditioning memory in marmoset monkeys. Behav. Processes 107, 163-166. doi:10.1016/j.beproc.2014.08.021.

Durkin, K., Rae, K., and Stritzke, W. G. K. (2012). The effect of images of thin and 
overweight body shapes on women's ambivalence towards chocolate. Appetite 58, 222-226. doi:10.1016/j.appet.2011.09.027.

Epstein, D. H., Preston, K. L., Stewart, J., and Shaham, Y. (2006). the Reinstatement Procedure. Psychopharmacology (Berl). 189, 1-16.

Fanous, S., Goldart, E. M., Theberge, F. R. M., Bossert, J. M., Shaham, Y., and Hope, B. T. (2012). Role of Orbitofrontal Cortex Neuronal Ensembles in the Expression of Incubation of Heroin Craving. J. Neurosci. 32, 11600-11609. doi:10.1523/JNEUROSCI.1914-12.2012.

Fauth, M., and Tetzlaff, C. (2016). Opposing Effects of Neuronal Activity on Structural Plasticity. Front. Neuroanat. 10, 75. doi:10.3389/fnana.2016.00075.

Feltmann, K., Giuliano, C., Everitt, B. J., Steensland, P., and Alsiö, J. (2018). The Effects of the Monoamine Stabilizer (-)-OSU6162 on Binge-Like Eating and CueControlled Food-Seeking Behavior in Rats. Neuropsychopharmacology 43, 617626. doi:10.1038/npp.2017.215.

Ferrario, C. R., Labouèbe, G., Liu, S., Nieh, E. H., Routh, V. H., Xu, S., et al. (2016). Homeostasis Meets Motivation in the Battle to Control Food Intake. J. Neurosci. 36, 11469-11481. doi:10.1523/JNEUROSCI.2338-16.2016.

Ferrario, C. R., Loweth, J. a., Milovanovic, M., Ford, K. a., Galiñanes, G. L., Heng, L. J., et al. (2011). Alterations in AMPA receptor subunits and TARPs in the rat nucleus accumbens related to the formation of $\mathrm{Ca} 2+$-permeable AMPA receptors during the incubation of cocaine craving. Neuropharmacology 61, 1141-1151.

doi:10.1016/j.neuropharm.2011.01.021.

Figlewicz, D. P., Bennett-Jay, J. L., Kittleson, S., Sipols, A. J., and Zavosh, A. (2011). Sucrose self-administration and CNS activation in the rat. Am. J. Physiol. Regul. Integr. Comp. Physiol. 300, R876-R884. doi:10.1152/ajpregu.00655.2010.

Flagel, S. B., Cameron, C. M., Pickup, K. N., Watson, S. J., Akil, H., and Robinson, T. E. (2011). A food predictive cue must be attributed with incentive salience for it to induce c-fos mRNA expression in cortico-striatal-thalamic brain regions. Neuroscience 196, 80-96. doi:10.1016/j.neuroscience.2011.09.004.

Forman, E. M., Hoffman, K. L., McGrath, K. B., Herbert, J. D., Brandsma, L. L., and Lowe, M. R. (2007). A comparison of acceptance- and control-based strategies for coping with food cravings: An analog study. Behav. Res. Ther. 45, 2372-2386. doi:10.1016/j.brat.2007.04.004.

Funk, D., Coen, K., Tamadon, S., Hope, B. T., Shaham, Y., and Lê, A. D. (2016). Role of Central Amygdala Neuronal Ensembles in Incubation of Nicotine Craving. $J$. Neurosci. 36, 8612-8623. doi:10.1523/JNEUROSCI.1505-16.2016.

García-García, I., Horstmann, a., Jurado, M. a., Garolera, M., Chaudhry, S. J., Margulies, D. S., et al. (2014). Reward processing in obesity, substance addiction and nonsubstance addiction. Obes. Rev. 15, 853-869. doi:10.1111/obr.12221.

Garcia, J., Kimeldorf, D. J., and Koellino, R. A. (1955). Conditioned Aversion to 
Saccharin Resulting from Exposure to Gamma Radiation. Am. Assoc. Adv. Sci. 122, 157-158.

Gearhardt, A. N., Rizk, M. T., and Treat, T. A. (2014). The association of food characteristics and individual differences with ratings of craving and liking. Appetite 79, 166-173. doi:10.1016/j.appet.2014.04.013.

Geary, N., and Smith, G. P. (1985). Pimozide decreases the positive reinforcing effect of sham fed sucrose in the rat. Pharmacol. Biochem. Behav. 22, 787-790. doi:10.1016/0091-3057(85)90528-3.

Gendall, K. a, Sullivan, P. F., Joyce, P. R., Fear, J. L., and Bulik, C. M. (1997). Psychopathology and personality of young women who experience food cravings. Addict. Behav. 22, 545-55. doi:http://dx.doi.org/10.1016/S0306-4603(96)00060-3.

Ghani, M. U., Mesadi, F., Kanık, S. D., Argunşah, A. Ö., Hobbiss, A. F., Israely, I., et al. (2016). Dendritic Spine Classification using Shape and Appearance Features based on Two-Photon Microscopy. J. Neurosci. Methods 279, 13-21. doi:10.1016/j.jneumeth.2016.12.006.

Glueck, E., Ginder, D., Hyde, J., North, K., and Grimm, J. W. (2016). Effects of dopamine D1 and D2 receptor agonists on environmental enrichment attenuated sucrose cue reactivity in rats. Psychopharmacology (Berl)., 815-825. doi:10.1007/s00213-016-4516-2.

Goff, B., Gee, D. G., Telzer, E. H., Humphreys, K. L., Gabard-Durnam, L., Flannery, J., et al. (2013). Reduced nucleus accumbens reactivity and adolescent depression following early-life stress. Neuroscience 249, 129-138. doi:10.1016/j.neuroscience.2012.12.010.

Goldstein, R. Z., and Volkow, N. D. (2011). Dysfunction of the prefrontal cortex in addiction: neuroimaging findings and clinical implications. Nat. Rev. Neurosci. 12, 652-669. doi:10.1038/nrn3119.

Grimm, J. W., Barnes, J. L., Koerber, J., Glueck, E., Ginder, D., Hyde, J., et al. (2016). Effects of acute or chronic environmental enrichment on regional Fos protein expression following sucrose cue-reactivity testing in rats. Brain Struct. Funct. 221, 2817-2830. doi:10.1007/s00429-015-1074-z.

Grimm, J. W., Barnes, J., North, K., Collins, S., and Weber, R. (2011a). A General Method for Evaluating Incubation of Sucrose Craving in Rats. J. Vis. Exp., 1-7. doi: $10.3791 / 3335$.

Grimm, J. W., Fyall, A. M., and Osincup, D. P. (2005). Incubation of sucrose craving: effects of reduced training and sucrose pre-loading. Physiol. Behav. 84, 73-79. doi:10.1016/j.physbeh.2004.10.011.Incubation.

Grimm, J. W., Harkness, J. H., Ratliff, C., Barnes, J., North, K., and Collins, S. (2011b). Effects of systemic or nucleus accumbens-directed dopamine D1 receptor antagonism on sucrose seeking in rats. Psychopharmacology (Berl). 216, 219-233. doi:10.1007/s00213-011-2210-y. 
Grimm, J. W., Hope, B. T., Wise, R. A., and Shaham, Y. (2001). Incubation of cocaine craving after withdrawal. Nature 412, 141.

Grimm, J. W., Lu, L., Hayashi, T., Hope, B. T., Su, T.-P., and Shaham, Y. (2003). Timedependent increases in brain-derived neurotrophic factor protein levels within the mesolimbic dopamine system after withdrawal from cocaine: implications for incubation of cocaine craving. $J$. Neurosci. 23, 742-747. doi:23/3/742 [pii].

Grimm, J. W., Manaois, M., Osincup, D., Wells, B., and Buse, C. (2007). Naloxone attenuates incubated sucrose craving in rats. Psychopharmacology (Berl). 194, 537544. doi:10.1007/s00213-007-0868-y.

Grimm, J. W., Shaham, Y., and Hope, B. T. (2002). Effect of cocaine and sucrose withdrawal period on extinction behavior, cue-induced reinstatement, and protein levels of the dopamine transporter and tyrosine hydroxylase in limbic and cortical areas in rats. Behav. Pharmacol. 13, 379-388.

doi:10.1016/j.biotechadv.2011.08.021.Secreted.

Groenewegen, H. J., der Zee, E. V. Van, te Kortschot, A., and Witter, M. P. (1987).

Organization of the projections from the subiculum to the ventral striatum in the rat. A study using anterograde transport of Phaseolus vulgaris leucoagglutinin. Neuroscience 23, 103-120. doi:10.1016/0306-4522(87)90275-2.

Guegan, T., Cutando, L., Gangarossa, G., Santini, E., Fisone, G., Martinez, A., et al. (2013). Operant behavior to obtain palatable food modified neuronal plasticity in the brain reward circuit. Eur. Neuropsychopharmacol. 23, 240-252. doi:10.1016/j.euroneuro.2012.04.009.

Guerra, L. G. G. C., and Silva, M. T. A. (2010). Learning processes and the neural analysis of conditioning. Psychol. Neurosci. 3, 195-208. doi:10.3922/j.psns.2010.2.xxx.

Guillem, K., Ahmed, S. H., and Peoples, L. L. (2014). Escalation of cocaine intake and incubation of cocaine seeking are correlated with dissociable neuronal processes in different accumbens subregions. Biol. Psychiatry 76, 31-39. doi:10.1016/j.biopsych.2013.08.032.

Haberny, S. L., Berman, Y., Meller, E., and Carr, K. D. (2004). Chronic food restriction increases D-1 dopamine receptor agonist-induced phosphorylation of extracellular signal-regulated kinase 1/2 and cyclic AMP response element-binding protein in caudate-putamen and nucleus accumbens. Neuroscience 125, 289-298. doi:10.1016/j.neuroscience.2004.01.037.

Hadad, N. A., and Knackstedt, L. A. (2014). Addicted to palatable foods: comparing the neurobiology of bulimia nervosa to that of drug addiction. Psychopharmacology (Berl). 231, 1897-1912. doi:10.1007/s00213-014-3461-1.Addicted.

Halbout, B., Bernardi, R. E., Hansson, A. C., and Spanagel, R. (2014). Incubation of cocaine seeking following brief cocaine experience in mice is enhanced by mGluR1 blockade. J. Neurosci. 34, 1781-90. doi:10.1523/JNEUROSCI.1076-14.2014. 
Hanley, J. G. (2014). Subunit-specific trafficking mechanisms regulating the synaptic expression of Ca2+-permeable AMPA receptors. Semin. Cell Dev. Biol. 27, 14-22. doi:10.1016/j.semcdb.2013.12.002.

Harkness, J. H., Webb, S., and Grimm, J. W. (2010). Abstinence-dependent transfer of lithium chloride-induced sucrose aversion to a sucrose-paired cue in rats.

Psychopharmacology (Berl). 208, 521-530. doi:10.1007/s00213-009-1755-5.

Harris, G. C., Hummel, M., Wimmer, M., Mague, S. D., and Aston-Jones, G. (2007). Elevations of FosB in the nucleus accumbens during forced cocaine abstinence correlate with divergent changes in reward function. Neuroscience 147, 583-591. doi:10.1016/j.neuroscience.2007.04.050.

Hebebrand, J., Albayrak, Ö., Adan, R., Antel, J., Dieguez, C., de Jong, J., et al. (2014). "Eating addiction", rather than "food addiction", better captures addictive-like eating behavior. Neurosci. Biobehav. Rev. 47, 295-306. doi:10.1016/j.neubiorev.2014.08.016.

Herrera, D. G., and Robertson, H. A. (1996). Activation of c-fos in the brain. Prog. Neurobiol. 50, 83-107.

Hill, A. J., and Heaton-Brown, L. (1994). The experience of food craving: A prospective investigation in healthy women. J. Psychosom. Res. 38, 801-814. doi:10.1016/00223999(94)90068-X.

Holahan, M. R., Clarke, M. J., and Hines, D. D. (2010). Dopamine-mediated MK-801induced elevation in food-based extinction responding in rats and associated changes in region-specific phosphorylated ERK. Psychopharmacology (Berl). 212, 393-403. doi:10.1007/s00213-010-1959-8.

Holahan, M. R., Westby, E. P., and Albert, K. (2012). Comparison of the MK-801induced appetitive extinction deficit with pressing for reward and associated pERK1/2 staining in prefrontal cortex and nucleus accumbens. Behav. Brain Res. 228, 194-202. doi:10.1016/j.bbr.2011.11.044.

Hollitt, S., Kemps, E., Tiggemann, M., Smeets, E., and Mills, J. S. (2010). Components of attentional bias for food cues among restrained eaters. Appetite 54, 309-313. doi:10.1016/j.appet.2009.12.005.

Hope, B. T., Nye, H. E., Kelz, M. B., Self, D. W., Iadarola, M. J., Nakabeppu, Y., et al. (1994). Induction of a long-lasting AP-1 complex composed of altered fos-like proteins in brain by chronic cocaine and other chronic treatments. Neuron 13, 12351244. doi:10.1016/0896-6273(94)90061-2.

Huang, X. F., Zavitsanou, K., Huang, X., Yu, Y., Wang, H. Q., Chen, F., et al. (2006). Dopamine transporter and D2 receptor binding densities in mice prone or resistant to chronic high fat diet-induced obesity. Behav. Brain Res. 175, 415-419. doi:10.1016/j.bbr.2006.08.034.

Huber, L., and Menzel, R. (2004). Structural basis of long-trm potentiation in single dendritic spines. Nature 429, 761-766. doi:10.1038/nature02594.1. 
Hussain, Z., and Totterdell, S. (1994). Calbindin-D28k imunoreactive neurons from two populations in the rat nucleus accumbens: a compartmental study. Brain Res 656, 191-198.

Hyman, S. E. (2005). Addiction: A Disease of Learning and Memory. Am. J. Psychiatry $162,1414-1422$.

Hyman, S. E., and Malenka, R. C. (2001). Addiction and the brain: the neurobiology of compulsion and its persistence. Nat. Rev. Neurosci. 2, 695-703. doi: $10.1038 / 35094560$.

Hyman, S. E., Malenka, R. C., and Nestler, E. J. (2006). NEURAL MECHANISMS OF ADDICTION: The Role of Reward-Related Learning and Memory. Annu. Rev. Neurosci. 29, 565-598. doi:10.1146/annurev.neuro.29.051605.113009.

Ito, R., Dalley, J. W., Howes, S. R., Robbins, T. W., and Everitt, B. J. (2000). Dissociation in conditioned dopamine release in the nucleus accumbens core and shell in response to cocaine cues and during cocaine-seeking behavior in rats. $J$. Neurosci. 20, 7489-7495. Available at: http://www.ncbi.nlm.nih.gov/entrez/query.fcgi? $\mathrm{cmd}=$ Retrieve $\& d b=P u b M e d \& d o p t=$ Citation\&list_uids $=11007908$.

Joffe, M. E., Grueter, C. A., and Grueter, B. A. (2015). Biological substrates of addiction. NIH Public Access 5, 151-171. doi:10.1002/wcs.1273.Biological.

Johnson, P. M., and Kenny, P. J. (2010). Dopamine D2 receptors in addiction-like reward dysfunction and compulsive eating in obese rats. Nat. Neurosci. 13, 635-641. doi:10.1038/nn.2519.

Karin, M., Liu, Z. G., and Zandi, E. (1997). AP-1 function and regulation. Curr. Opin. Cell Biol. 9, 240-246. doi:10.1016/S0955-0674(97)80068-3.

Kasai, H., Fukuda, M., Watanabe, S., Hayashi-Takagi, A., and Noguchi, J. (2010). Structural dynamics of dendritic spines in memory and cognition. Trends Neurosci. 33, 121-129. doi:10.1016/j.tins.2010.01.001.

Kelley, A. E., and Berridge, K. C. (2002). The neuroscience of natural rewards: relevance to addictive drugs. J. Neurosci. 22, 3306-3311. doi:20026361.

Kelz, M. B., Chen, J., Carlezon Jr., W. a, Whisler, K., Gilden, L., Beckmann, a M., et al. (1999). Expression of the transcription factor deltaFosB in the brain controls sensitivity to cocaine. Nature 401, 272-276. doi:10.1038/45790.

Kelz, M. B., and Nestler, E. J. (2000). deltaFosB: a molecular switch underlying longterm neural plasticity. Curr. Opin. Neurol. 13, 715-720. doi:10.1097/00019052200012000-00017.

Kesner, R. P., and Churchwell, J. C. (2011). An analysis of rat prefrontal cortex in mediating executive function. Neurobiol. Learn. Mem. 96, 417-31. doi:10.1016/j.nlm.2011.07.002.

Kessels, H. W., and Malinow, R. (2009). Synaptic AMPA Receptor Plasticity and 
Behavior. Neuron 61, 340-350. doi:10.1016/j.neuron.2009.01.015.

Kobrin, K. L., Moody, O., Arena, D. T., Moore, C. F., Heinrichs, S. C., and Kaplan, G. B. (2016). Acquisition of morphine conditioned place preference increases the dendritic complexity of nucleus accumbens core neurons. Addict. Biol. 21, 10861096. doi:10.1111/adb.12273.

Kolb, B., and Whishaw, I. Q. (1998). Brain Plasticity and Behavior. Annu. Rev. Psy 49, 43-64. doi:10.1146/annurev.psych.49.1.43.

Koob, G. F. (2000). Neurobiology of addiction. Toward the development of new therapies. Ann. N. Y. Acad. Sci. 909, 170-185. doi:10.1111/j.17496632.2000.tb06682.x.

Koob, G. F., and Volkow, N. D. (2010). Neurocircuitry of addiction. Neuropsychopharmacology 35, 217-238. doi:10.1038/npp.2010.4.

Koya, E., Spijker, S., Voorn, P., Binnekade, R., Schmidt, E. D., Schoffelmeer, A. N. M., et al. (2006). Enhanced cortical and accumbal molecular reactivity associated with conditioned heroin, but not sucrose-seeking behaviour. J. Neurochem. 98, 905-915. doi:10.1111/j.1471-4159.2006.03917.x.

Koya, E., Uejima, J. L., Wihbey, K. a., Bossert, J. M., Hope, B. T., and Shaham, Y. (2009a). Role of ventral medial prefrontal cortex in incubation of cocaine craving. Neuropharmacology 56 Suppl 1, 177-85. doi:10.1016/j.neuropharm.2008.04.022.

Koya, E., Uejima, J. L., Wihbey, K. a., Bossert, J. M., Hope, B. T., and Shaham, Y. (2009b). Role of ventral medial prefrontal cortex in incubation of cocaine craving. Neuropharmacology 56, 177-185. doi:10.1016/j.neuropharm.2008.04.022.

Krasnova, I. N., Marchant, N. J., Ladenheim, B., McCoy, M. T., Panlilio, L. V., Bossert, J. M., et al. (2014a). Incubation of methamphetamine and palatable food craving after punishment-induced abstinence. Neuropsychopharmacology 39, 2008-2016. doi:10.1038/npp.2014.50.

Krasnova, I. N., Marchant, N. J., Ladenheim, B., McCoy, M. T., Panlilio, L. V, Bossert, J. M., et al. (2014b). Incubation of Methamphetamine and Palatable Food Craving after Punishment-Induced Abstinence. Neuropsychopharmacology 39, 1-24. doi:10.1038/npp.2014.50.

Krasnova, I. N., Marchant, N. J., Ladenheim, B., McCoy, M. T., Panlilio, L. V, Bossert, J. M., et al. (2014c). Incubation of Methamphetamine and Palatable Food Craving after Punishment-Induced Abstinence. Neuropsychopharmacology 39, 2008-2016. doi:10.1038/npp.2014.50.

Krasnova, I. N., Marchant, N. J., Ladenheim, B., McCoy, M. T., Panlilio, L. V, Bossert, J. M., et al. (2014d). Incubation of Methamphetamine and Palatable Food Craving after Punishment-Induced Abstinence. Neuropsychopharmacology 39, 2008-2016. doi:10.1038/npp.2014.50.

Kreek, M. J., LaForge, K. S., and Butelman, E. (2002). Pharmacotherapy of addictions. Nat. Rev. Drug Discov. 1, 710-726. doi:10.1038/nrd897. 
Langleben, D. D., Ruparel, K., Elman, I., Busch-Winokur, S., Pratiwadi, R., Loughead, J., et al. (2008). Acute effect of methadone maintenance dose on brain fMRI response to heroin-related cues. Am. J. Psychiatry 165, 390-394.

doi:10.1176/appi.ajp.2007.07010070.

Larson, E. B., Akkentli, F., Edwards, S., Graham, D. L., Simmons, D. L., Alibhai, I. N., et al. (2010). Striatal regulation of DeltaFosB, FosB, and cFos during cocaine selfadministration and withdrawal. J. Neurochem. 115, 112-122. doi:10.1111/j.14714159.2010.06907.x.

Lee, B. R., Ma, Y.-Y., Huang, Y. H., Wang, X., Otaka, M., Ishikawa, M., et al. (2013). Maturation of silent synapses in amygdala-accumbens projection contributes to incubation of cocaine craving. Nat. Neurosci. 16, 1644-51. doi:10.1038/nn.3533.

Li, C., and Frantz, K. J. (2009). Attenuated incubation of cocaine seeking in male rats trained to self-administer cocaine during periadolescence. Psychopharmacology (Berl). 204, 725-733. doi:10.1007/s00213-009-1502-y.

Li, X., Caprioli, D., and Marchant, N. J. (2014). Recent updates on incubation of drug craving: a mini-review. Addict. Biol. doi:10.1111/adb.12205.

Li, X., DeJoseph, M. R., Urban, J. H., Bahi, a., Dreyer, J.-L., Meredith, G. E., et al. (2013). Different Roles of BDNF in Nucleus Accumbens Core versus Shell during the Incubation of Cue-Induced Cocaine Craving and Its Long-Term Maintenance. $J$. Neurosci. 33, 1130-1142. doi:10.1523/JNEUROSCI.3082-12.2013.

Li, X., Rubio, F. J., Zeric, T., Bossert, J. M., Kambhampati, S., Cates, H. M., et al. (2015a). Incubation of Methamphetamine Craving Is Associated with Selective Increases in Expression of Bdnf and Trkb, Glutamate Receptors, and Epigenetic Enzymes in Cue-Activated Fos-Expressing Dorsal Striatal Neurons. J. Neurosci. 35, 8232-8244. doi:10.1523/JNEUROSCI.1022-15.2015.

Li, X., and Wolf, M. E. (2011). Brain-derived neurotrophic factor rapidly increases AMPA receptor surface expression in rat nucleus accumbens. Eur. J. Neurosci. 34, 190-198. doi:10.1111/j.1460-9568.2011.07754.x.

Li, X., Zeric, T., Kambhampati, S., Bossert, J. M., and Shaham, Y. (2015b). The Central Amygdala Nucleus is Critical for Incubation of Methamphetamine Craving. Neuropsychopharmacology, 1-10. doi:10.1038/npp.2014.320.

Li, Y.-Q., Li, F.-Q., Wang, X.-Y., Wu, P., Zhao, M., Xu, C.-M., et al. (2008). Central amygdala extracellular signal-regulated kinase signaling pathway is critical to incubation of opiate craving. J. Neurosci. 28, 13248-13257. doi:10.1523/JNEUROSCI.3027-08.2008.

Loi, B., Fantini, N., Colombo, G., Gessa, G. L., Riva, A., Bombardelli, E., et al. (2013). Reducing effect of an extract of Phaseolus vulgaris on food intake in mice - Focus on highly palatable foods. Fitoterapia 85 , 14-19. doi:10.1016/j.fitote.2012.12.015.

Loweth, J. A., Tseng, K. Y., and Wolf, M. E. (2014). Adaptations in AMPA receptor transmission in the nucleus accumbens contributing to incubation of cocaine 
craving. Neuropharmacology 76, 287-300. doi:10.1016/j.neuropharm.2013.04.061.

Lu, L., Grimm, J. W., Hope, B. T., and Shaham, Y. (2004). Incubation of cocaine craving after withdrawal: a review of preclinical data. Neuropharmacology 47 Suppl 1, 214 26. doi:10.1016/j.neuropharm.2004.06.027.

Lu, L., Hope, B. T., Dempsey, J., Liu, S. Y., Bossert, J. M., and Shaham, Y. (2005). Central amygdala ERK signaling pathway is critical to incubation of cocaine craving. Nat. Neurosci. 8, 212-9. doi:10.1038/nn1383.

Lu, L., Uejima, J. L., Gray, S. M., Bossert, J. M., and Shaham, Y. (2007). Systemic and Central Amygdala Injections of the mGluR2/3 Agonist LY379268 Attenuate the Expression of Incubation of Cocaine Craving. Biol. Psychiatry 61, 591-598. doi:10.1016/j.biopsych.2006.04.011.

Lubbers, B. R., Matos, M. R., Horn, A., Visser, E., Van der Loo, R. C., Gouwenberg, Y., et al. (2015). The Extracellular Matrix Protein Brevican Limits Time-Dependent Enhancement of Cocaine Conditioned Place Preference. Neuropsychopharmacology, 1-10. doi:10.1038/npp.2015.361.

Lubman, D. I., Yüeel, M., and Pantelis, C. (2004). Addiction, a condition of compulsive behaviour? Neuroimaging and neuropsychological evidence of inhibitory dysregulation. Addiction 99, 1491-1502. doi:10.1111/j.1360-0443.2004.00808.x.

Ma, Y.-Y., Lee, B. R., Wang, X., Guo, C., Liu, L., Cui, R., et al. (2014). Bidirectional Modulation of Incubation of Cocaine Craving by Silent Synapse-Based Remodeling of Prefrontal Cortex to Accumbens Projections. Neuron, 1453-1467. doi:10.1016/j.neuron.2014.08.023.

Malinow, R., and Malenka, R. C. (2002). AMPA receptor trafficking and synaptic plasticity. Annu. Rev. Neurosci. 25, 103-26.

doi:10.1146/annurev.neuro.25.112701.142758.

Mancino, S., Mendonça-Netto, S., Martín-García, E., and Maldonado, R. (2016). Role of DOR in neuronal plasticity changes promoted by food-seeking behaviour. Addict. Biol. doi:10.1111/adb.12401.

Mandt, B. H., Allen, R. M., and Zahniser, N. R. (2009). Individual differences in initial low-dose cocaine-induced locomotor activity and locomotor sensitization in adult outbred female Sprague-Dawley rats. Pharmacol. Biochem. Behav. 91, 511-516. doi:10.1016/j.pbb.2008.09.002.

Mason, A. (2009). NIH Public Access. 19, 389-399. doi:10.1016/j.asieco.2008.09.006.EAST.

Mathes, W. F., Brownley, K. A., Mo, X., and Bulik, C. M. (2009). The biology of binge eating. Appetite 52, 545-553. doi:10.1016/j.appet.2009.03.005.

Mavridis, I., Boviatsis, E., and Anagnostopoulou, S. (2011). The human nucleus accumbens suffers parkinsonism-related shrinkage: A novel finding. Surg. Radiol. Anat. 33, 595-599. doi:10.1007/s00276-011-0802-1. 
Mayer, M. L., Westbrook, G. L., and Guthrie, P. B. (1984). Voltage-dependent block by Mg2+ of NMDA responses in spinal cord neurons. Nature 309, 261-263.

McClung, C. a., Ulery, P. G., Perrotti, L. I., Zachariou, V., Berton, O., and Nestler, E. J. (2004a). ??fosB: A molecular switch for long-term adaptation in the brain. Mol.

Brain Res. 132, 146-154. doi:10.1016/j.molbrainres.2004.05.014.

McClung, C. A., Ulery, P. G., Perrotti, L. I., Zachariou, V., Berton, O., and Nestler, E. J. (2004b). DeltaFosB: a molecular switch for long-term adaptation in the brain. Mol. Brain Res. 132, 146-54. doi:10.1016/j.molbrainres.2004.05.014.

Mendoza, J., Angeles-Castellanos, M., and Escobar, C. (2005). Differential role of the accumbens Shell and Core subterritories in food-entrained rhythms of rats. Behav. Brain Res. 158, 133-142. doi:10.1016/j.bbr.2004.08.016.

Meredith, G. E., Agolia, R., Arts, M. P. M., Groenewegen, H. J., and Zahm, D. S. (1992). Morphological differences between projection neurons of the core and shell in the nucleus-accumbens of the rat. Neuroscience 50, 149-162.

Meredith, G. E., Callen, S., and Scheuer, D. A. (2002). Brain-derived neurotrophic factor expression is increased in the rat amygdala, piriform cortex and hypothalamus following repeated amphetamine administration. Brain Res. 949, 218-227. doi:10.1016/S0006-8993(02)03160-8.

Meule, A. (2015). Back By Popular Demand: A narrative review on the history of food addiction research. 88, 295-302.

Meule, A., and Gearhardt, A. (2014). Food Addiction in the Light of DSM-5. Nutrients 6, 3653-3671. doi:10.3390/nu6093653.

Meule, A., and Kübler, A. (2012). Food cravings in food addiction: The distinct role of positive reinforcement. Eat. Behav. 13, 252-255. doi:10.1016/j.eatbeh.2012.02.001.

Meyer, M. D., Risbrough, V. B., Liang, J., and Boutelle, K. N. (2014). Pavlovian conditioning to hedonic food cues in overweight and lean individuals. Appetite $87 \mathrm{C}$, 56-61. doi:10.1016/j.appet.2014.12.002.

Morris, M. J., Beilharz, J. E., Maniam, J., Reichelt, A. C., and Westbrook, R. F. (2015). Why is obesity such a problem in the 21 st century? The intersection of palatable food, cues and reward pathways, stress, and cognition. Neurosci. Biobehav. Rev. 58, 36-45. doi:10.1016/j.neubiorev.2014.12.002.

Mueller, D., Perdikaris, D., and Stewart, J. (2002). Persistence and drug-induced reinstatement of a morphine-induced conditioned place preference. 136, 389-397.

Mueller, D., and Stewart, J. (2000). Cocaine-induced conditioned place preference : reinstatement by priming injections of cocaine after extinction. 115, 39-47.

Muller, D., and Unterwald, E. (2005). D1 dopamine receptors modulate DeltaFosB induction in rat striatum after intermittent morphine administration. J. Pharmacol. Exp. Ther. 314, 148-154. doi:10.1124/jpet.105.083410.3-hydroxy-5-methyl-4isoxazolepropionic. 
Mumberg, D., Lucibello, F. C., Schuermann, M., and Müller, R. (1991). Alternative splicing of fosB transcripts results in differentially expressed mRNAs encoding functionally antagonistic proteins. Genes Dev. 5, 1212-1223. doi:10.1101/gad.5.7.1212.

Nägerl, U. V., Eberhorn, N., Cambridge, S. B., and Bonhoeffer, T. (2004). Bidirectional Activity-Dependent Morphological Plasticity in Hippocampal Neurons. 44, 759767.

Nasser, J. A., Bradley, L. E., Leitzsch, J. B., Chohan, O., Fasulo, K., Haller, J., et al. (2011). Psychoactive effects of tasting chocolate and desire for more chocolate. Physiol. Behav. 104, 117-121. doi:10.1016/j.physbeh.2011.04.040.

Nasser, J. A., Evans, S. M., Geliebter, A., Pi-Sunyer, F. X., and Foltin, R. W. (2008). Use of an operant task to estimate food reinforcement in adult humans with and without BED. Obesity (Silver Spring). 16, 1816-20. doi:10.1038/oby.2008.281.

Nathan, P. E., Conrad, M., and Skinstad, A. H. (2015). History of the Concept of Addiction. Annu. Rev. Clin. Psychol. 12, annurev-clinpsy-021815-093546. doi:10.1146/annurev-clinpsy-021815-093546.

Nestler, E. J. (2004). Molecular mechanisms of drug addiction. Neuropharmacology 47, 24-32. doi:10.1016/j.neuropharm.2004.06.031.

Nestler, E. J. (2005). The neurobiology of cocaine addiction. Sci. Pract. Perspect. 3, 410. doi:10.1151/spp05314.

Nestler, E. J. (2008). Transcriptional mechanisms of addiction: role of FosB. Philos. Trans. R. Soc. B Biol. Sci. 363, 3245-3255. doi:10.1098/rstb.2008.0067.

Nestler, E. J. (2012). Transcriptional mechanisms of drug addiction. Clin. Psychopharmacol. Neurosci. 10, 136-43. doi:10.9758/cpn.2012.10.3.136.

Nestler, E. J. (2015). $\triangle$ FosB: A transcriptional regulator of stress and antidepressant responses. Eur. J. Pharmacol. 753, 66-72. doi:10.1016/j.ejphar.2014.10.034.

Nestler, E. J., Barrot, M., and Self, D. W. (2001). DeltaFosB: a sustained molecular switch for addiction. Proc. Natl. Acad. Sci. U. S. A. 98, 11042-6. doi:10.1073/pnas.191352698.

Nestler, E. J., Hope, B. T., and Widnell, K. L. (1993). Drug addiction: A model for the molecular basis of neural plasticity. Neuron 11, 995-1006. doi:10.1016/08966273(93)90213-B.

Newton, T. F., De La Garza, R., Kalechstein, A. D., Tziortzis, D., and Jacobsen, C. a (2009). Theories of addiction: methamphetamine users' explanations for continuing drug use and relapse. Am. J. Addict. 18, 294-300. doi:10.1080/10550490902925920.

Nicoll, R., and Malenka, R. (1999). Expression Mechanisms Underlying NMDA Receptor-Dependent Long-Term Potentiation. Ann. New York Acad. ..., 515-525. Available at: http://onlinelibrary.wiley.com/doi/10.1111/j.17496632.1999.tb11320.x/full [Accessed October 7, 2013]. 
Nowak, L., Bregestovski, P., and Ascher, P. (1984). Magnesum gates glutamate-activated channels in mouse central neurons. Nature 307, 462-465.

Noye Tuplin, E. W., Lightfoot, S. H. M., and Holahan, M. R. (2018). Comparison of the time-dependent changes in immediate early gene labeling and spine density following abstinence from contingent or non-contingent chocolate pellet delivery. Front. Behav. Neurosci. In Press.

Nugent, A. L., Anderson, E. M., Larson, E. B., and Self, D. W. (2017). Incubation of cueinduced reinstatement of cocaine, but not sucrose, seeking in $\mathrm{C} 57 \mathrm{BL} / 6 \mathrm{~J}$ mice. Pharmacol. Biochem. Behav. 159, 12-17. doi:10.1016/j.pbb.2017.06.017.

Nyberg, F. (2014). Structural plasticity of the brain to psychostimulant use. Neuropharmacology 87, 115-124. doi:10.1016/j.neuropharm.2014.07.004.

Obrien, C. P., Childress, A. R., Mclellan, A. T., and Ehrman, R. (1992). Classical Conditioning in Drug-Dependent Humans. Ann. N. Y. Acad. Sci. 654, 400-415.

Ogden, C. L., Carroll, M. D., Kit, B. K., and Flegal, K. M. (2014). Prevalence of childhood and adult obesity in the United States, 2011-2012. Jama 311, 806-814. doi:10.1001/jama.2014.732.

Oginsky, M. F., Goforth, P. B., Nobile, C. W., Lopez-Santiago, L. F., and Ferrario, C. R. (2016). Eating 'Junk-Food' Produces Rapid and Long-Lasting Increases in NAc CPAMPA Receptors: Implications for Enhanced Cue-Induced Motivation and Food Addiction. Neuropsychopharmacology 41, 2977-2986. doi:10.1038/npp.2016.111.

Okamoto, K.-I., Nagai, T., Miyawaki, A., and Hayashi, Y. (2004). Rapid and persistent modulation of actin dynamics regulates postsynaptic reorganization underlying bidirectional plasticity. Nat. Neurosci. 7, 1104-1112. doi:10.1038/nn1311.

Olausson, P. (2006). FosB in the Nucleus Accumbens Regulates Food-Reinforced Instrumental Behavior and Motivation. J. Neurosci. 26, 9196-9204. doi:10.1523/JNEUROSCI.1124-06.2006.

Orsini, C., Bonito-Oliva, A., Montanari, C., Conversi, D., and Cabib, S. (2013). Partial extinction of a conditioned context enhances preference for elements previously associated with cocaine but not with chocolate. Physiol. Behav. 120, 1-10. doi:10.1016/j.physbeh.2013.06.021.

Parkinson, J. a, Olmstead, M. C., Burns, L. H., Robbins, T. W., and Everitt, B. J. (1999). Dissociation in effects of lesions of the nucleus accumbens core and shell on appetitive pavlovian approach behavior and the potentiation of conditioned reinforcement and locomotor activity by D-amphetamine. J. Neurosci. 19, 24012411. Available at:

http://www.ncbi.nlm.nih.gov/entrez/query.fcgi?cmd=Retrieve\&db=PubMed\&dopt= Citation\&list_uids=10066290.

Patrono, E., Segni, M. Di, Patella, L., Andolina, D., Valzania, A., Latagliata, E. C., et al. (2015). When chocolate seeking becomes compulsion: Gene-environment interplay. PLoS One 10, 1-21. doi:10.1371/journal.pone.0120191. 
Pavlov, I. P. (2010). Conditioned reflexes: an investigation of the physiological activity of the cerebral cortex. Ann. Neurosci. 17, 136-141.

Peng, X.-X., Ziff, E. B., and Carr, K. D. (2011a). Effects of food restriction and sucrose intake on synaptic delivery of AMPA receptors in nucleus accumbens. Synapse 65, 1024-31. doi:10.1002/syn.20931.

Peng, X. X., Ziff, E. B., and Carr, K. D. (2011b). Effects of food restriction and sucrose intake on synaptic delivery of AMPA receptors in nucleus accumbens. Synapse 65, 1024-1031. doi:10.1002/syn.20931.

Pérez-Ortiz, J. M., Galiana-Simal, A., Salas, E., González-Martín, C., García-Rojo, M., and Alguacil, L. F. (2016). A high-fat diet combined with food deprivation increases food seeking and the expression of candidate biomarkers of addiction. Addict. Biol., n/a-n/a. doi:10.1111/adb.12389.

Peters, J., Kalivas, P. W., and Quirk, G. J. (2009). Extinction circuits for fear and addiction overlap in prefrontal cortex. Learn. Mem. 16, 279-88. doi:10.1101/1m.1041309.

Pickens, C. L., Airavaara, M., Theberge, F., Fanous, S., Hope, B. T., and Shaham, Y. (2011). Neurobiology of the incubation of drug craving. Trends Neurosci. 34, 41120. doi:10.1016/j.tins.2011.06.001.

Piper, M. E. (2015). Withdrawal: Expanding a Key Addiction Construct. Nicotine Tob. Res., ntv048-. doi:10.1093/ntr/ntv048.

Pitchers, K. K., Vialou, V., Nestler, E. J., Laviolette, S. R., Lehman, M. N., and Coolen, L. M. (2013). Natural and Drug Rewards Act on Common Neural Plasticity Mechanisms with FosB as a Key Mediator. J. Neurosci. 33, 3434-3442. doi:10.1523/JNEUROSCI.4881-12.2013.

Placanica, J. L., Faunce, G. J., and Soames Job, R. F. (2002). The effect of fasting on attentional biases for food and body shape/weight words in high and low eating disorder inventory scorers. Int. J. Eat. Disord. 32, 79-90. doi:10.1002/eat.10066.

Pothos, E. N., Creese, I., and Hoebel, B. G. (1995). Restricted eating with weight loss selectively decreases extracellular dopamine in the nucleus accumbens and alters dopamine response to amphetamine, morphine, and food intake. J. Neurosci. 15, 6640-6650. Available at:

http://www.ncbi.nlm.nih.gov/entrez/query.fcgi? $\mathrm{cmd}=$ Retrieve \&db=PubMed\&dopt= Citation\&list_uids $=7472425$.

Purgianto, A., Weinfeld, M. E., and Wolf, M. E. (2016). Prolonged withdrawal from cocaine self-administration affects prefrontal cortex- and basolateral amygdalanucleus accumbens core circuits but not accumbens GABAergic local interneurons. Addict. Biol. doi:10.1111/adb.12430.

Quick, S. L., Pyszczynski, A. D., Colston, K. A., and Shahan, T. A. (2011). Loss of alternative non-drug reinforcement induces relapse of cocaine-seeking in rats: Role of dopamine D 1 receptors. Neuropsychopharmacology 36, 1015-1020. 
doi:10.1038/npp.2010.239.

Quintero, G. C. (2013). Role of nucleus accumbens glutamatergic plasticity in drug addiction. Neuropsychiatr. Dis. Treat. 9, 1499-1512. doi:10.2147/NDT.S45963.

Quirk, G. J., and Mueller, D. (2008). Neural mechanisms of extinction learning and retrieval. Neuropsychopharmacology 33, 56-72. doi:10.1038/sj.npp.1301555.

Renthal, W., Carle, T. L., Maze, I., Covington, H. E., Truong, H.-T., Alibhai, I., et al. (2008). FosB Mediates Epigenetic Desensitization of the c-fos Gene After Chronic Amphetamine Exposure. J. Neurosci. 28, 7344-7349. doi:10.1523/JNEUROSCI.1043-08.2008.

Robinson, T. E., and Berridge, K. C. (1993). The neural basis of drug craving: An incentive-sensitization theory of addiction. Brain Res. Rev. 18, 247-291. doi:10.1016/0165-0173(93)90013-P.

Robinson, T. E., and Berridge, K. C. (2000). The psychology and neurobiology of addiction: an incentive-sensitization view. Addiction 95, 91-117. doi:doi:10.1046/j.1360-0443.95.8s2.19.x.

Robinson, T. E., and Berridge, K. C. (2008). Review. The incentive sensitization theory of addiction: some current issues. Philos. Trans. R. Soc. Lond. B. Biol. Sci. 363, 3137-3146. doi:10.1098/rstb.2008.0093.

Robinson, T. E., and Kolb, B. (1999). Alterations in the morphology of dendrites and dendritic spines in the nucleus accumbens and prefrontal cortex following repeated treatment with amphetamine or cocaine. Eur. J. Neurosci. 11, 1598-1604. doi:10.1046/j.1460-9568.1999.00576.x.

Robinson, T. E., and Kolb, B. (2004). Structural plasticity associated with exposure to drugs of abuse. Neuropharmacology 47, 33-46. doi:10.1016/j.neuropharm.2004.06.025.

Robison, A. J., and Nestler, E. J. (2011). Transcriptional and epigenetic mechanisms of addiction. Nat. Rev. Neurosci. 12, 623-637. doi:10.1038/nrn3111.

Rochefort, N. L., and Konnerth, A. (2012). Dendritic spines: from structure to in vivo function. EMBO Rep. 13, 699-708. doi:10.1038/embor.2012.102.

Roedel, A., Storch, C., Holsboer, F., and Ohl, F. (2006). Effects of light or dark phase testing on behavioural and cognitive performance in DBA mice. Lab. Anim. 40, 371-381. doi:10.1258/002367706778476343.

Rogers, P. J., and Smit, H. J. (2000). Food craving and food "addiction": A critical review of the evidence from a biopsychosocial perspective. Pharmacol. Biochem. Behav. 66, 3-14. doi:10.1016/S0091-3057(00)00197-0.

Salamone, J., and Correa, M. (2002). Motivational views of reinforcement: Implications for understanding the behavioural functions of nucleus accumbens dopamine. Behav. Brain Res. 137, 3-25. 
Salamone, J. D., Correa, M., Farrar, A., and Mingote, S. M. (2007). Effort-related functions of nucleus accumbens dopamine and associated forebrain circuits. Psychopharmacology (Berl). 191, 461-482. doi:10.1007/s00213-006-0668-9.

Salgado, S., and Kaplitt, M. G. (2015). The Nucleus Accumbens: A Comprehensive Review. Stereotact. Funct. Neurosurg., 75-93. doi:10.1159/000368279.

Sayette, M. A. (2016). The Role of Craving in Substance Use Disorders: Theoretical and Methodological Issues. Annu. Rev. Clin. Psychol. 12, 407-433. doi:10.1146/annurev-clinpsy-021815-093351.

Scheyer, A. F., Loweth, J. A., Christian, D. T., Uejima, J., Rabei, R., Le, T., et al. (2016). AMPA Receptor Plasticity in Accumbens Core Contributes to Incubation of Methamphetamine Craving. Biol. Psychiatry 80, 661-670. doi:10.1016/j.biopsych.2016.04.003.

Schroeder, B. E., Binzak, J. M., and Kelley, A. E. (2001). A common profile of prefrontal cortical activation following exposure to nicotine- or chocolate-associated contextual cues. Neuroscience 105, 535-545. doi:10.1016/S0306-4522(01)00221-4.

Schüz, B., Schüz, N., and Ferguson, S. G. (2015). It's the power of food: Individual differences in food cue responsiveness and snacking in everyday life. Int. J. Behav. Nutr. Phys. Act. 12, 1-8. doi:10.1186/s 12966-015-0312-3.

Semba, J., Sakai, M., Miyoshi, R., Mataga, N., Fukamauchi, F., and Kito, S. (1996). Differential expression of c-fos mRNA in rat prefrontal cortex, striatum, N. accumbens and lateral septum after typical and atypical antipsychotics: an in situ hybridization study. Neurochem. Int. 29, 435-442.

Serafine, K. M., Bentley, T. A., Grenier, A. E., and France, C. P. (2014). Eating high fat chow and the behavioral effects of direct-acting and indirect-acting dopamine receptor agonists in female rats. Behav. Pharmacol. 25, 287-295. doi:10.1097/FBP.0000000000000052.

Shalev, U., Morales, M., Hope, B., Yap, J., and Shaham, Y. (2001). Time-dependent changes in extinction behavior and stress-induced reinstatement of drug seeking following withdrawal from heroin in rats. Psychopharmacology (Berl). 156, 98-107. doi:10.1007/s002130100748.

Sharma, S., Fernandes, M., and Fulton, S. (2013). Adaptations in brain reward circuitry underlie palatable food cravings and anxiety induced by high-fat diet withdrawal. Int. J. Obes. 37, 1183-1191.

Sheng, M., and Kim, M. J. (2002). Postsynaptic signaling and plasticity mechanisms. Science 298, 776-80. doi:10.1126/science.1075333.

Shepard, J. D., Bossert, J. M., Liu, S. Y., and Shaham, Y. (2004). The anxiogenic drug yohimbine reinstates methamphetamine seeking in a rat model of drug relapse. Biol. Psychiatry 55, 1082-1089. doi:10.1016/j.biopsych.2004.02.032.

Shirayama, Y., and Chaki, S. (2006). Neurochemistry of the nucleus accumbens and its relevance to depression and antidepressant action in rodents. Curr. Neuropharmacol. 
4, 277-291. doi:10.2174/157015906778520773.

Sinha, R. (2013). The clinical neurobiology of drug craving. Curr. Opin. Neurobiol. 23, 649-654. doi:10.1016/j.conb.2013.05.001.

Small, D. M., Jones-Gotman, M., and Dagher, A. (2003). Feeding-induced dopamine release in dorsal striatum correlates with meal pleasantness ratings in healthy human volunteers. Neuroimage 19, 1709-1715. doi:10.1016/S1053-8119(03)00253-2.

Spiga, S., Mulas, G., Piras, F., and Diana, M. (2014). The "addicted" spine. Front. Neuroanat. 8, 1-7. doi:10.3389/fnana.2014.00110.

Staddon, J. E. R., and Cerutti, D. T. (2003). Operant Conditioning. Annu. Rev. Psychol. 51,115-144. doi:10.1016/j.micinf.2011.07.011.Innate.

Stamp, J. A., Mashoodh, R., van Kampen, J. M., and Robertson, H. A. (2008). Food restriction enhances peak corticosterone levels, cocaine-induced locomotor activity, and $\Delta$ FosB expression in the nucleus accumbens of the rat. Brain Res. 1204, 94101. doi:10.1016/j.brainres.2008.02.019.

Stawski, P., Janovjak, H., and Trauner, D. (2010). Pharmacology of ionotropic glutamate receptors: A structural perspective. Bioorg. Med. Chem. 18, 7759-7772. doi:10.1016/j.bmc.2010.09.012.

Stice, E., Spoor, S., Bohon, C., Veldhuizen, M. G., and Small, D. M. (2008). Relation of reward from food intake and anticipated food intake to obesity: a functional magnetic resonance imaging study. J. Abnorm. Psychol. 117, 924-35. doi: $10.1037 / \mathrm{a} 0013600$.

Stice, E., Yokum, S., Blum, K., and Bohon, C. (2010). Weight Gain Is Associated with Reduced Striatal Response to Palatable Food. J. Neurosci. 30, 13105-13109. doi:10.1523/JNEUROSCI.2105-10.2010.

Stoeckel, L. E., Weller, R. E., Cook, E. W., Twieg, D. B., Knowlton, R. C., and Cox, J. E. (2008). Widespread reward-system activation in obese women in response to pictures of high-calorie foods. Neuroimage 41, 636-647. doi:10.1016/j.neuroimage.2008.02.031.

Stratford, T. R., and Kelley, A. E. (1997). GABA in the nucleus accumbens shell participates in the central regulation of feeding behavior. J. Neurosci. 17, 4434-40.

Sun, Y., Pan, Z., and Ma, Y. (2017). Increased entrances to side compartments indicate incubation of craving in morphine-induced rat and tree shrew CPP models. Pharmacol. Biochem. Behav. 159, 62-68. doi:10.1016/j.pbb.2017.07.007.

Svoboda, K., Tank, D. W., and Denk, W. (2017). Direct Measurement of Coupling between Dendritic Spines and Shafts Author ( s ): Karel Svoboda, David W . Tank and Winfried Denk Published by: American Association for the Advancement of Science Stable URL : http://www.jstor.org/stable/2889445 JSTOR is . 272, 716719.

Tada, T., and Sheng, M. (2006). Molecular mechanisms of dendritic spine 
morphogenesis. Curr. Opin. Neurobiol. 16, 95-101.

doi:10.1016/j.conb.2005.12.001.

Teegarden, S. L., Nestler, E. J., and Bale, T. L. (2009). DeltaFosB-mediated alterations in dopamine signaling are normalized by a palatable high fat diet. 64, 941-950.

doi:10.1016/j.biopsych.2008.06.007.

Tellez, L. A., Ferreira, J. G., Medina, S., Land, B. B., Dileone, R. J., and De Araujo, I. E. (2013). Flavor-independent maintenance, extinction, and reinstatement of fat selfadministration in mice. Biol. Psychiatry 73, 851-859.

doi:10.1016/j.biopsych.2013.02.028.

Terrier, J., Luscher, C., and Pascoli, V. (2015). Cell-Type Specific Insertion of GluA2Lacking AMPARs with Cocaine Exposure Leading to Sensitization, Cue-Induced Seeking and Incubation of Craving. Neuropsychopharmacology 41, 1-11. doi:10.1038/npp.2015.345.

Theberge, F. R. M., Pickens, C. L., Goldart, E., Fanous, S., Hope, B. T., Liu, Q. R., et al. (2012). Association of time-dependent changes in mu opioid receptor mRNA, but not $\mathrm{BDNF}$, TrkB, or MeCP2 mRNA and protein expression in the rat nucleus accumbens with incubation of heroin craving. Psychopharmacology (Berl). 224, 559-571. doi:10.1007/s00213-012-2784-z.

Thiel, K. J., Pentkowski, N. S., Peartree, N. a., Painter, M. R., and Neisewander, J. L. (2010). Environmental living conditions introduced during forced abstinence alter cocaine-seeking behavior and Fos protein expression. Neuroscience 171, 11871196. doi:10.1016/j.neuroscience.2010.10.001.

Thomas, J. E., Caballero, J., and Harrington, C. A. (2015). The Incidence of Akathisia in the Treatment of Schizophrenia with Aripiprazole, Asenapine and Lurasidone: A Meta-Analysis. Curr. Neuropharmacol. 13, 681-691. doi:10.2174/1570159X13666150115220221.

Tiffany, S. T., and Wray, J. M. (2012). The clinical significance of drug craving. Ann. N. Y. Acad. Sci. 1248, 1-17. doi:10.1111/j.1749-6632.2011.06298.x.

Traversy, G., and Chaput, J.-P. (2015). Alcohol Consumption and Obesity: An Update. Curr. Obes. Rep. 4, 122-130. doi:10.1007/s13679-014-0129-4.

Tuplin, E. W., Stocco, M. R., and Holahan, M. R. (2015). Attenuation of MK-801Induced Behavioral Perseveration by Typical and Atypical Antipsychotic Pretreatment in Rats. 129, 399-411.

Tzschentke, T. M. (2007). Measuring reward with the conditioned place preference (CPP) paradigm: Update of the last decade. Addict. Biol. 12, 227-462. doi:10.1111/j.1369-1600.2007.00070.x.

Uejima, J. L., Bossert, J. M., Poles, G. C., and Lu, L. (2007). Systemic and central amygdala injections of the mGluR 2 / 3 agonist LY379268 attenuate the expression of incubation of sucrose craving in rats. 181, 292-296.

doi:10.1016/j.bbr.2007.04.019. 
Ulery, P. G. (2006). Regulation of FosB Stability by Phosphorylation. J. Neurosci. 26, 5131-5142. doi:10.1523/JNEUROSCI.4970-05.2006.

Usuda, I., Tanaka, K., and Chiba, T. (1998). Efferent projections of the nucleus accumbens in the rat with special reference to subdivision of the nucleus: Biotinylated dextran amine study. Brain Res. 797, 73-93. doi:10.1016/S00068993(98)00359-X.

Van den Oever, M. C. (2012). Glutamate receptors in synaptic assembly and plasticity: case studies on fly NMJs. doi:10.1007/978-3-7091-0932-8_1.

van Huijstee, A. N., and Mansvelder, H. D. (2015). Glutamatergic synaptic plasticity in the mesocorticolimbic system in addiction. Front. Cell. Neurosci. 8, 1-13. doi:10.3389/fncel.2014.00466.

Veilleux, J. C., and Skinner, K. D. (2015). Smoking, food, and alcohol cues on subsequent behavior: A qualitative systematic review. Clin. Psychol. Rev. 36, 13-27. doi:10.1016/j.cpr.2015.01.001.

Velázquez-Sánchez, C., Ferragud, A., Moore, C. F., Everitt, B. J., Sabino, V., and Cottone, P. (2014a). High Trait Impulsivity Predicts Food Addiction-Like Behavior in the Rat. Neuropsychopharmacology, 1-10. doi:10.1038/npp.2014.98.

Velázquez-Sánchez, C., Ferragud, A., Moore, C. F., Everitt, B. J., Sabino, V., and Cottone, P. (2014b). High trait impulsivity predicts food addiction-like behavior in the rat. Neuropsychopharmacology 39, 2463-2472. doi:10.1038/npp.2014.98.

Venniro, M., Caprioli, D., and Y, S. (2016). Animal models of drug relapse and craving: From drug priming-induced reinstatement to incubation of craving after voluntary abstinence. Prog. Brain Res. 335, 25-52.

Venniro, M., Zhang, M., Shaham, Y., and Caprioli, D. (2017). Incubation of Methamphetamine but not Heroin Craving after Voluntary Abstinence in Male and Female Rats. Neuropsychopharmacology 42, 1126-1135. doi:10.1038/npp.2016.287.

Wang, G.-J. M., Nora D. Volkow, M., Panayotis K. Thanos, P., and Joanna S. Fowler, P. (2009). Imaging of Brain Dopamine Pathways: Implications for Understanding Obesity. J Addict Med. 3, 8-18. doi:10.1097/ADM.0b013e31819a86f7.Imaging.

Wang, G.-J., Volkow, N. D., Thanos, Panayotis, K., and Fowler, J. S. (2004). Similarity between obesity and drug addiction as assessed by neurofunctional imaging. $J$. Addict. Dis. 23, 39-53. doi:10.1300/J069v23n03.

Wang, Y., Cesena, T. I., Ohnishi, Y., Burger-caplan, R., Lam, V., Kirchho, P. D., et al. (2012). Small Molecule Screening Identi fi es Regulators of the Transcription Factor $\Delta$ FosB $\dagger$.

Wanigaratne, S. (2006). Psychology of addiction. Psychiatry 5, 455-460. doi:10.1053/j.mppsy.2006.09.007.

Watson, P., Wiers, R. W., Hommel, B., and De Wit, S. (2014). Working for food you 
don't desire. Cues interfere with goal-directed food-seeking. Appetite 79, 139-148. doi:10.1016/j.appet.2014.04.005.

Weiss, F. (2005). Neurobiology of craving, conditioned reward and relapse. Curr. Opin. Pharmacol. 5, 9-19. doi:10.1016/j.coph.2004.11.001.

Willard, S. S., and Koochekpour, S. (2013). Glutamate, glutamate receptors, and downstream signaling pathways. Int. J. Biol. Sci. 9, 948-959. doi:10.7150/ijbs.6426.

Wilson, C. J., Groves, P. M., Kitai, S. T., and Linder, J. C. (1983). Three-dimensional structure of dendritic spines in the rat neostriatum. J Neurosci 3, 383-8. Available at:

http://www.ncbi.nlm.nih.gov/entrez/query.fcgi? $\mathrm{cmd}=$ Retrieve\&db=PubMed\&dopt= Citation\&list_uids $=6822869$.

Wise, R. a. (1996). Neurobiology of addiction. Curr. Opin. Neurobiol. 6, 243-251. doi:10.1016/S0959-4388(96)80079-1.

Wise, R. A. (1988). The neurobiology of craving: implications for the understanding and treatment of addiction. J. Abnorm. Psychol. 97, 118-132. doi:10.1037/0021843X.97.2.118.

Wise, R. A. (2004). Dopamine, learning and motivation. Nat. Rev. Neurosci. 5, 483-494. doi:10.1038/nrn1406.

Wise, R. A. (2006). Role of brain dopamine in food reward and reinforcement. Philos. Trans. R. Soc. Lond. B. Biol. Sci. 361, 1149-1158. doi:10.1098/rstb.2006.1854.

Wise, R. A., Fotuhi, M., and Colle, L. M. (1989). Facilitation of feeding by nucleus accumbens amphetamine injections: Latency and speed measures. Pharmacol. Biochem. Behav. 32, 769-772. doi:10.1016/0091-3057(89)90031-2.

Wise, R. A., Spindler, J., and Legault, L. (1978). Major Attenuation of Food Reward with Performance-Sparing Doses of Pimozide in the Rat. Can. J. Psychol. 32, 77-85. doi:10.1037/h0081678.

Wise, R. a, and Rompre, P. P. (1989). Brain dopamine and reward. Annu. Rev. Psychol. 40, 191-225. doi:10.1146/annurev.psych.40.1.191.

Wolf, M. E. (2010). Dysregulation of AMPA receptor transmission in the nucleus accumbens in animal models of cocaine addiction. Neurotox Res 18, 393-409. doi:10.1007/s12640-010-9176-0.Dysregulation.

Wolf, M. E., and Ferrario, C. R. (2010). AMPA receptor plasticity in the nucleus accumbens after repeated exposure to cocaine. Neurosci. Biobehav. Rev. 35, 185211. doi:10.1016/j.neubiorev.2010.01.013.

Wolf, M. E., and Tseng, K. Y. (2012). Calcium-permeable AMPA receptors in the VTA and nucleus accumbens after cocaine exposure: when, how, and why? Front. Mol. Neurosci. 5, 1-27. doi:10.3389/fnmol.2012.00072.

Wong, E. H. F., Knight, A. R., and Woodruff, G. N. (1988). [3H]MK-801 Labels a site 
on the n-mthyl-d-aspartate receptor channel complex in rat brain membranes. $J$. Neurochem. 50, 274-281.

Wright, C. I., Beijer, a V, and Groenewegen, H. J. (1996). Basal amygdaloid complex afferents to the rat nucleus accumbens are compartmentally organized. J. Neurosci. $16,1877-1893$.

Wright, C. I., and Groenewegen, H. J. (1996). Patterns of overlap and segregation between insular cortical, intermediodorsal thalamic and basal amygdaloid afferents in the nucleus accumbens of the rat. Neuroscience 73, 359-373. doi:10.1016/03064522(95)00592-7.

Yasoshima, Y., Scott, T. R., and Yamamoto, T. (2006). Memory-dependent c-fos expression in the nucleus accumbens and extended amygdala following the expression of a conditioned taste aversive in the rat. Neuroscience 141, 35-45. doi:10.1016/j.neuroscience.2006.03.019.

Yasumatsu, N., Matsuzaki, M., Miyazaki, T., Noguchi, J., and Kasai, H. (2008). Principles of Long-Term Dynamics of Dendritic Spines. J. Neurosci. 28, 1359213608. doi:10.1523/JNEUROSCI.0603-08.2008.

Záborszky, L., Alheid, G. F., Beinfeld, M. C., Eiden, L. E., Heimer, L., and Palkovits, M. (1985). Cholecystokinin innervation of the ventral striatum: A morphological and radioimmunological study. Neuroscience 14. doi:10.1016/0306-4522(85)90302-1.

Zahm, D. S., and Brog, J. S. (1992). On the significance of subterritories in the "accumbens" part of the rat ventral striatum. Neuroscience 50, 751-767. doi:10.1016/0306-4522(92)90202-D.

Zheng, D., Cabeza de Vaca, S., Jurkowski, Z., and Carr, K. D. (2015). Nucleus accumbens AMPA receptor involvement in cocaine-conditioned place preference under different dietary conditions in rats. Psychopharmacology (Berl). 232, 23132322. doi:10.1007/s00213-015-3863-8.

Zombeck, J. A., Chen, G. T., Johnson, Z. V., Rosenberg, D. M., Craig, A. B., and Rhodes, J. S. (2008). Neuroanatomical specificity of conditioned responses to cocaine versus food in mice. Physiol. Behav. 93, 637-650. doi:10.1016/j.physbeh.2007.11.004.

Zucker, R. S., and Regehr, W. G. (2002). Short-Term Synaptic Plasticity. Annu. Rev. Physiol. 64, 355-405. doi:10.1146/annurev.physiol.64.092501.114547. 